\author{
Universidade de São Paulo \\ Instituto de Física
}

\title{
Modelos Microscópicos para Cristais Líquidos Nemáticos
}

\author{
Eduardo dos Santos Nascimento
}

Orientador: Prof. Dr. André de Pinho Vieira Coorientador: Prof. Dr. Silvio R. Salinas

Tese de doutorado apresentada ao Instituto de Física da Universidade de São Paulo, como requisito parcial para a obtenção do título de Doutor em Ciências.

Banca Examinadora:

Prof. Dr. André de Pinho Vieira - Orientador (IFUSP)

Prof. Dr. Cristiano Luís Pinto de Oliveira (IFUSP)

Prof. Dr. Mario José de Oliveira (IFUSP)

Prof. Dr. Ítalo Nunes de Oliveira (UFAL)

Prof. Dr. Luiz Roberto Evangelista (UEM)

São Paulo 


\section{FICHA CATALOGRÁFICA \\ Preparada pelo Serviço de Biblioteca e Informação do Instituto de Física da Universidade de São Paulo}

Nascimento, Eduardo dos Santos

Modelos microscópicos para cristais líquidos nemáticos.

São Paulo, 2018.

Tese (Doutorado) - Universidade de São Paulo. Instituto de Física. Depto. de Física Geral.

Orientador: Prof. Dr. André de Pinho Vieira

Área de Concentração: Física da Matéria Condensada

Unitermos: 1. Cristais líquidos; 2. Modelos de mecânica estatística; 3. Mudança de fase; 4. Física teórica. 


\section{Agradecimentos}

- Ao professor André de Pinho Vieira pela orientação, incentivo e dedicação ao longo desses anos.

- Ao professor Silvio Salinas pela ajuda e dicussões sobre os diversos temas ligados ao projeto.

- Ao professor Peter Palffy-Muhoray pela colaboração e oportunidade de passar um ano na Kent State University (EUA).

- Aos companheiros com os quais convivi no IFUSP.

- À FAPESP (processo 2013/12873-9) pelo suporte financeiro. 



\section{Resumo}

Neste trabalho estudamos, no contexto de campo médio, modelos microscópicos que possam descrever o comportamento termodinâmico das fases nemáticas em sistemas líquidocristalinos. Considerando apenas interações atrativas, investigamos modelos de interações quadrupolares para objetos intrinsecamente biaxiais. Esses modelos apresentam mesofases nemáticas uniaxiais e biaxiais, pontos triplos e multicríticos (tricríticos, pontos de Landau, etc.). Ainda no contexto de forças atrativas, introduzimos um modelo de mistura binária de objetos intrinsecamente uniaxiais e objetos intrinsecamente biaxiais, numa formulação annealed. Essa mistura apresenta diagramas de fases bastante ricos, com topologias diversas, onde identificamos estruturas uniaxiais e biaxiais, fases reentrantes e uma grande variedade de pontos multicríticos (tricríticos, pontos críticos terminais, etc.). No caso de interações estéricas, estudamos uma teoria do funcional densidade para sistemas anisotrópicos densos, construída a partir de uma aproximação de van der Waals. Para esferoides prolatos, o modelo prevê um espaço de orientações com regiões não-acessíveis para as partículas. Além disso, o sistema apresenta uma região de coexistência entre as fases nemática e isotrópica.

Palavras-chave: cristais líquidos, transições nemáticas, modelos microscópicos, modelo de Maier-Saupe, modelo de Onsager 



\section{Abstract}

We study, in a mean-field approximation, microscopic models which can lead to nematic liquid-crystalline phases. Considering attractive forces, we investigate models with quadrupolar interactions for intrinsically biaxial objects. These models present uniaxial and biaxial nematic mesophases, triple and multicritical points (tricritical point, Landau point, etc.). We also introduce a model for a binary mixture of intrinsically uniaxiail and biaxial objects, in an annealed treatment. The mixture exhibits phase diagrams with very rich topologies, where we find uniaxial and biaxial structures, reentrant phases and many different multicritical behaviors (tricritical point, critical endpoint, etc.). Moreover, assuming steric interactions, we investigate a density functional theory for hard anisotropic bodies at high densities, based on a van der Waals approximation. For hard spheroids, the model leads to an orientation space with forbidden regions for the particles. Also, the system phase separates in a nematic and an isotropic phases.

Keywords: liquid crystals, nematic transitions, microscopic models, Maier-Saupe theory, Onsager theory 



\section{Sumário}

Agradecimentos

Resumo v v

Abstract vii

\begin{tabular}{lll}
\hline & Introdução & 1
\end{tabular}

2 Modelos microscópicos com interações atrativas 9

2.1 Interações de dispersão $\ldots \ldots \ldots$. . . . . . . . . . . . . . . . . . . . . 9

2.1 .1 Tratamento geral da energia de dispersão . . . . . . . . . . . . . . 10

2.1 .2 Moléculas uniaxiais . . . . . . . . . . . . . . . . . . 15

2.2 Interações de dispersão em moléculas intrinsecamente biaxiais . . . . . . . 17

2.2 .1 Conexão com a teoria de Landau-de Gennes . . . . . . . . . . . . . 23

2.3 Modelos de Straley e SVD . . . . . . . . . . . . . . . . . . . . . . 25

2.3 .1 Modelo de Straley . . . . . . . . . . . . . . . . . . . 25

2.3 .2 Modelo SVD . . . . . . . . . . . . . . . . . . 26

3 Modelo para mistura binária de objetos uniaxiais e biaxiais 33

3.1 Misturas binárias e fases nemáticas . . . . . . . . . . . . . . . . . . . 33

3.2 Mistura binária de objetos uniaxiais e biaxiais . . . . . . . . . . . . . . 35

3.3 Conexão com a teoria de Landau-de Gennes . . . . . . . . . . . . . . . . . 38

3.4 Diagramas de fases . . . . . . . . . . . . . . . . . . . . 40

\begin{tabular}{|lll}
\hline & Interações estéricas e nemáticos uniaxiais & 49
\end{tabular}

4.1 Sistemas de muitos corpos rígidos . . . . . . . . . . . . . . . . . . . . . . . 49 
4.2 Modelo de "bastões" rígidos . . . . . . . . . . . . . . . . . . . . . . 52

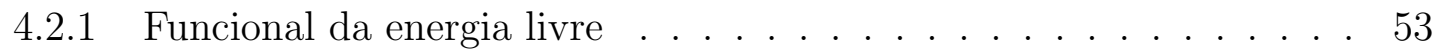

$4.2 .2 \quad$ Distribuição estacionária e equações de estado . . . . . . . . . . . . 56

4.2 .3 Transição nemática-isotrópica num sistema de cilindros longos . . . 58

4.3 Teoria do funcional densidade para nemáticos em altas densidades . . . . . 62

$4.3 .1 \quad$ Funcional da energia livre $\ldots \ldots \ldots$. . . . . . . . . . . 62

4.3 .2 Distribuição estacionária para esferóides prolatos . . . . . . . . . 66

$4.3 .3 \quad$ Equações de estado e transição nemático-isotrópica $\quad$. . . . . . . . . . 69

\begin{tabular}{lll}
\hline 5 & Conclusões e Perspectivas & 73
\end{tabular}

\begin{tabular}{ll}
\hline Referências Bibliográficas & 75
\end{tabular}

\begin{tabular}{|ll}
\hline Apêndices. & 79
\end{tabular}

\begin{tabular}{|lll}
\hline A & Representação irredutível de um tensor simétrico & 79
\end{tabular}

\begin{tabular}{lr}
\hline Apêndices. & 81
\end{tabular}

\begin{tabular}{ll|l}
\hline B & Publicações & 81
\end{tabular} 


\section{Lista de Figuras}

2.1 A interação efetiva depende da orientação relativa dos mesógenos e do vetor posição relativa $\vec{R}$ que conectada os centros de massa. . . . . . . . . . . . 14

2.2 Representação esquemática de uma molécula intrinsecamente uniaxial que tem a forma efetiva de um cilindro prolato. O estado orientacial é determinado pelo diretor local $\vec{n} . \ldots \ldots \ldots \ldots$. . . . . . . . . . 15

2.3 Representação esquemática de uma molécula intrinsecamente biaxial. O estado orientacial da molécula é especificado pelo sistema de referência

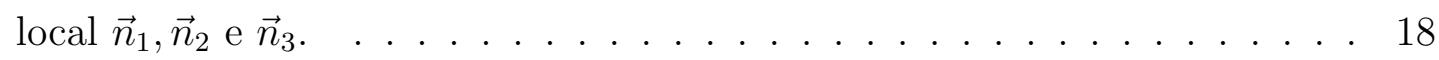

2.4 Discretização dos estados orientacionais para um objeto intrinsecamente biaxial. . . . . . . . . . . . . . . . . . . 20

2.5 Diagrama de fases do modelo Maier-Saupe discretizado, em termos da temperatura reduzida $T$ e da biaxialidade $\Delta$. ISO: isotrópica. $\mathrm{N}_{+}$: uniaxial calamítica. $\mathrm{N}_{+}$: uniaxial discótica. B: biaxial. L: ponto de Landau. . . . . 22

2.6 Níveis de energia do modelo Maier-Saupe discretizado para moléculas intrinsecamente biaxiais. . . . . . . . . . . . . . . . . . . 23

2.7 Diagrama de fases do modelo SVD discretizado, em termos da temperatura reduzida $T$ e do parâmetro $\lambda$. I: isotrópica. U: uniaxial. B: biaxial. O modelo apresenta pontos tricrítico e triplo, mas não tem ponto de Landau. 31

2.8 Diagrama de fases do modelo SVD discretizado, mostrando a região onde aparece um segundo ponto tricrítico. . . . . . . . . . . . . . . . . . 32

3.1 Objetos com formas distintas e eixos maiores orientados paralelamente. . . 36

3.2 Objetos com formas diferentes e eixos maiores orientados perpendicularmente. 36

3.3 Diagrama de fases para $\mu=5 . \ldots \ldots \ldots \ldots$. . . . . . . . . . . 40 
$3.4 \quad$ Diagrama de fases para $\mu=-5 . \ldots \ldots \ldots \ldots \ldots \ldots$

$3.5 \quad$ Diagrama de fases da mistura binária para $\Delta=1 . \ldots \ldots \ldots \ldots$

3.6 Níveis de energia da mistura binária como função do parâmetro de biaxialidade $\Delta$. As degenerescências dos níveis são indicadas por $\omega . \quad \ldots \ldots .41$

$3.7 \quad$ Diagrama de fases para $\Delta=1 . \ldots \ldots \ldots \ldots \ldots \ldots$

3.8 Diagrama de fases para $\Delta=1.4 \ldots \ldots \ldots \ldots \ldots \ldots$

3.9 Diagrama de fases para $\Delta=1.54 \ldots \ldots \ldots \ldots \ldots \ldots$

3.10 Diagrama de fases para $\Delta=1.57 . \ldots \ldots \ldots \ldots \ldots \ldots$

3.11 Pontos multicríticos no plano $T \times \Delta . \ldots \ldots \ldots \ldots \ldots$

3.12 Diagrama de fases para $\Delta=2.9 . \ldots \ldots \ldots \ldots \ldots$

3.13 Diagrama de fases para $\Delta=3 . \ldots \ldots \ldots \ldots \ldots$

3.14 Pontos multicríticos no plano $T \times \Delta . \ldots \ldots \ldots \ldots \ldots \ldots$

3.15 Diagrama de fases para $\Delta=6 . \ldots \ldots \ldots \ldots \ldots$

4.1 Representação esquemática de objetos anisotrópicos prolatos. (a) esferocilindro de dimensões típicas $L$ e $D$. (b) esferóide com semi-eixos $a$ e $b$.

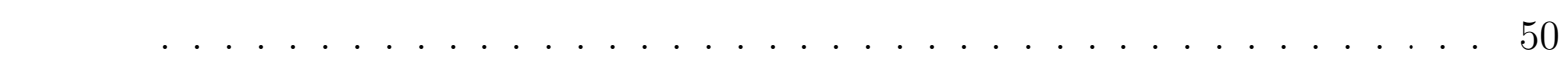

4.2 Diagrama de fases obtido por Bolhuis e Frenkel para um sistema de esferocilindros rígidos de dimensões típicas $L$ e $D$, onde $\rho^{*}$ é uma densidade

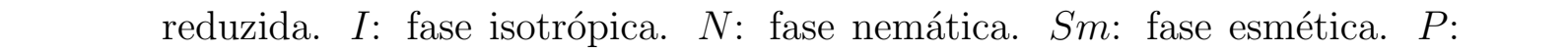
sólido plástico. $A A A$ e $A B C$ : fases sólidas. Regiões hachuradas: coexistência de fases. . . . . . . . . . . . . . . . . . . . . 51

4.3 Diagrama de fases descrito por Frenkel e Mulder para um sistema de esferóides rígidos de dimensões típicas $2 a$ e $2 b$, onde $\rho^{*}$ é uma densidade \begin{tabular}{|l|l|}
\hline reduzida e $x=a / b$ é a relação de aspecto. $I$ : fase isotrópica. $N$ : fase \\
\hline
\end{tabular} \begin{tabular}{|l|l|}
\hline nemática. $P S$ : sólido plástico. S: fase sólida. Regiões hachuradas: coe- \\
\hline
\end{tabular} xistência de fases. . . . . . . . . . . . . . . . . . . . . 52

4.4 Representação de uma configuração de dois cilindros circulares, de tamanhos característicos $L$ e $D$, e com orientação relativa $\gamma$. . . . . . . . . 59

4.5 Parâmetro $S \times$ fração volumétrica $\bar{\eta}$. Linhas pretas: $\quad$ soluções estáveis. Linhas vermelhas: soluções não-estáveis ( metaestáveis e/ou instáveis). . . 61 
4.6 Pressão $\Pi \times$ fração volumétrica $\bar{\eta}$. Linhas pretas: soluções estáveis. Linhas vermelhas: soluções não-estáveis ( metaestáveis e/ou instáveis). Linha azul: coexistência de fases. $I$ : fase isotrópica. $N$ : fase nemática. . . . . . . . . 61

4.7 Representação esquemática de uma configuração de dois elipsóides idênticos, de tamanhos característicos $L$ e $W$, e com orientação relativa $\gamma$. . . . . . . 67

$4.8 S \times \phi$ para soluções uniaxiais. Linhas pretas: soluções estáveis. Linhas verdes: soluções metaestáveis. Linhas vermelhas: soluções instáveis. . . . . 71

4.9 Coexistência de fases. (a) Tangente dupla. (b) Construção de Maxwell. I:

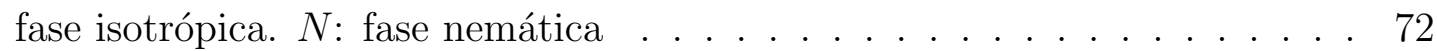




\section{Capítulo 1}

\section{Introdução}

É bastante difundida, mesmo no meio acadêmico, a ideia de que a matéria possui três estados: sólido, líquido e vapor. No caso particular de sólidos cristalinos, os objetos microscópicos que formam tal fase estão organizados numa estrutura espacial periódica, o que leva à rigidez característica dos sólidos. Por outro lado, tanto no estado líquido como no vapor as partículas têm maior facilidade para se movimentarem, o que resulta na fluidez típica dessas fases. Outro ponto muito importante é que sólidos e fluidos têm, por exemplo, propriedades elétricas e magnéticas diferentes, o que está associado ao tipo de ordenamento orientacional das partículas.

Todavia, certos materiais se comportam de tal forma que desafiam a visão de que só existem três estados da matéria. De fato, os chamamos "cristais líquidos" são fases que exibem propriedades comuns a sólidos e líquidos [1; 2; 3]. Como são estruturas intemediárias, os cristais líquidos também são denomimados mesofases. É comum chamar de mesógenos os constituintes elementares (moléculas, agregados moleculares, etc) dos sistemas líquido-cristalinos. No geral, esses materiais apresentam fluidez, mas também possuem propriedades macroscópicas anisotrópicas, como anisotropias ótica e dielétrica [1; 4]. Devido a essas peculiaridades, as mesofases da matéria são o foco constante de investigações teóricas e experimentais, sendo que também há bastante interesse do ponto de vista de aplicações [3].

As fases de cristais líquidos podem ser influenciadas tanto por processos térmicos quanto pela ação de solventes [1; 2; 3]. De um modo geral, dizemos que um material líquido-cristalino é termotrópico quando a temperatura é o parâmetro termodinâmico relevante para o sistema. Os termotrópicos geralmente são formados por moléculas ani- 
sotrópicas, com formas efetivas prolatas, oblatas, ou até mesmo com simetrias mais reduzidas, em que os mesógenos têm forma de banana ou de bumerangue [3]. Por outro lado, nos chamados cristais líquidos liotrópicos a concentração (número de partículas por volume) é o parâmetro macroscópico que influencia as propriedades de tais materiais, embora a temperatura também possa ser relevante. Exemplos de sistemas liotrópicos são misturas de moléculas anfifílicas dissolvidas num solvente [2; 4].

Quanto ao tipo de organização, os cristais líquidos exibem uma grande variedade de estruturas onde os mesógenos apresentam ordem nas orientacões e até mesmo nas posições dos centros de massa [1; 2; 3]. De fato, existem várias maneiras de como os mesógenos podem se organizar e discutir todas elas em detalhes seria bem extenso, de modo que sugerimos ao leitor referências espefíficas da área, tais como os livros de de Gennes e Prost [1] e Singh [2]. Neste trabalho focaremos apenas nas chamadas mesofases nemáticas. Nessas estruturas, os constituintes fundamentais podem se orientar, em média, ao longo de uma ou mais direções preferenciais, sem que haja ordenamento posicional de longo alcance. Em particular, os denomimados nemáticos uniaxiais apresentam somente uma direção preferencial de ordenamento, descrita por um vetor unitário, chamado diretor nemático, em torno do qual o sistema tem simetria rotacional.

Nemáticos uniaxiais são provavelmente as fases líquido-cristalinas mais simples encontradas na natureza. No entanto, é possível obter estruturas nemáticas mais complicadas, permitindo, por exemplo, que essas estruturas tenham simetrias mais reduzidas [1]. Uma situação de grande interesse ocorre quando há quebra de simetria rotacional em torno do diretor de uma fase uniaxial [2]. Isso pode levar a mesofases nemáticas biaxiais. Tais fases de cristais líquidos são caracterizadas por exibirem, na prática, três direções preferenciais de ordenamento. É importante mencionar que, na realidade, a maioria dos mesógenos não têm simetria cilíndrica [5]. Logo, seria mais apropriado representá-los usando objetos anisotrópicos tais como paralelepípedos ou elipsoides triaxiais. Dentro dessa perspectiva, uma fase biaxial poderia surgir, em condições termodinâmicas favoráveis, quando os eixos principais correspondentes dos mesógenos tivessem, em média, as mesmas orientações.

Do ponto de vista teórico, estruturas biaxiais foram previstas pela primeira vez no trabalho de Freiser [6; 7], que discute a possibilidade de tais fases surgirem num sistema formado por objetos assimétricos interagindo por meio de forças atrativas. Todavia, a 
primeira verificação experimental só veio dez anos depois com Yu e Saupe [8], que estudaram uma mistura liotrópica ternária de KL/1-decanol/ $\mathrm{D}_{2} \mathrm{O}$. Nesse tipo de mistura, a fase biaxial aparece entre duas fases uniaxiais, geralmente denominadas calamítica e discótica. Atualmente, existem vários resultados experimentais envolvendo mesofases biaxiais em liotrópicos. Por exemplo, Akpinar e colaboradores [9; 10] mostram a existência de fases biaxiais em misturas liotrópicas quaternárias. Uma discussão mais elaborada sobre os aspectos experimentais envolvendo liotrópicos é apresentada por Figueiredo Neto e Galerne, no capítulo 11 do livro editado por Luckhurst e Sluckin [11].

Somente em meados da década de 2000 é que apareceram os primeiros resultados experimentais sobre fases nemáticas biaxiais em cristais líquidos termotrópicos. Madsen et al. [12] e Acharya et al. [13] afirmam ter encontrado estruturas biaxiais estáveis num sistema formado por moléculas com forma efetiva de "banana". Note que mesógenos desse tipo são menos simétricos do que aqueles discutidos no trabalho de Freiser [6; 7], que considerou objetos com simetria de um paralelepípedo retângulo. Ainda no contexto experimental, Merkel et al. [14] também afirmam ter detectado fases biaxiais num sistema constituído por moléculas tetrápodes achatadas. É importante mencionar que, apesar das evidências experimentais, ainda existe muito debate sobre o design de moléculas que possam levar a mesofases biaxiais estáveis. Isso se deve ao fato de que, na prática, o sistema sofre transições para outras fases líquido-cristalinas, e até mesmo fases sólidas, antes de chegar numa estrutura nemática biaxial.

É fundamental o elaboração de modelos microscópicos que possam levar a uma melhor compreensão das fases nemáticas biaxiais. Um dos nossos interesses é estudar situações que permitam um tratamento estatístico simples mas que sejam capazes de descrever estruturas biaxiais. Assim, no capítulo 2, discutiremos alguns modelos para sistemas constituídos por mesógenos intrinsecamente biaxiais. Em particular, focaremos nossa atenção em objetos que interagem através de forças atrativas. Por conta disso, começaremos discutindo como são as interações de dispersão para objetos que não têm simetria esférica. Então, tomando a parte anisotrópica dessas interações, chegaremos num modelo de interações quadrupolares, na linha do trabalho de Freiser [6; 7]. De fato, isso nos permitirá escrever o modelo de Maier-Saupe para mesógenos biaxiais com orientações discretas. Esse modelo tem um diagrama de fases com estruturas uniaxiais e biaxiais e um ponto 
multicrítico de Landau.

Em seguida, apresentaremos a teoria desenvolvida por Straley [15], que introduziu um potencial de pares muito geral, levando em conta apenas as propriedades orientacionais de mesógenos. Esse modelo se reduz ao caso descrito pelas forças de dispersão quando impomos certas condições sobre os parâmetros. Contudo, por ser mais geral, a interação de Straley pode levar a diagramas de fases diferentes daquele obtido pela teoria de MaierSaupe. De fato, quase trinta anos depois, o modelo de Straley foi reescrito por Sonnet, Virga e Durand [16] com o auxílio de uma linguagem matemática mais elegante. Mais que isso, os autores, para um certo conjunto de parâmetros, obtiveram um diagrama de fases com pontos triplos e tricríticos, mas que não apresenta ponto de Landau. Iremos definir um modelo de campo médio do sistema descrito por Sonnet, Virga e Durand e admitir que os mesógenos têm orientações restritas aos eixos cartesianos. Então, mostraremos que esse modelo leva a resultados consistentes qualitativamente com o modelo original.

Vale a pena mencionar que Alben [17] chega a um diagrama de fases semelhante ao obtido por $\mathrm{Yu}$ e Saupe [8], a partir de um modelo de mistura binária de discos e cilindros. Esse resultado sugere que misturas envolvendo objetos com formas distintas podem apresentar um comportamento termodinâmico muito rico. Obviamente, existem muitas maneiras de definir esses sistemas, pois precisamos definir os tipos de objetos em questão, tais como cilindros, esferoides ou paralelepípedos. Também devemos especificar a maneira de lidar com os graus de liberdade que descrevem as formas dos mesógenos. Apesar de todos os detalhes envolvendo o problema, misturas são modelos simples que permitem estabelecer vários resultados teóricos para fases nemáticas.

Uma questão muito investigada e debatida no caso das misturas binárias é a estabilidade de mesofases nemáticas biaxiais. Adotando um potencial de interação do tipo Maier-Saupe, Palffy et al. [18, 19] preveem que uma mistura de discos e cilindros não tem fase biaxial estável caso uma condição associada às interações, denominada de "média geométria", seja satisfeita. Um ponto importante desse trabalho é que os graus de liberdade de forma são variáveis móveis (recozidas), o que é razoável em física de fluidos. Posteriormente, um tratamento alternativo foi desenvolvido por Henriques e Henriques [20], que estudaram um sistema constituído de objetos com simetria cilíndrica em que a forma é uma variável desordenada fixa (temperada), como ocorre em física de sólidos. 
Dentro dessa abordagem, os autores obtiveram um diagrama de fases no qual há uma estrutra biaxial termodinamicamente estável entre duas fases uniaxiais, e que é consistente qualitativamente com o trabalho de Yu e Saupe [8]. Todas essas questões foram debatidas e esclarecidas por do Carmo et al. [21], em que os autores mostram que um ponto fundamental nesse problema é o tratamento estatístico usado para lidar com as variáveis associadas ao tipo de objeto. Supreendentemente, esse trabalho prevê que é possível haver fase biaxial estável caso os graus de liberdade de forma sejam descritos por variáveis aleatórias parcialmente recozidas.

Embora haja muitos resultados envolvendo mesógenos uniaxiais e formas diferentes, é perfeitamente possível considerar situações nas quais os objetos têm simetrias mais reduzidas. Dentro de uma perspectiva de campo médio, Longa et al. [22] estudaram um sistema de mesógenos biaxiais no qual os graus de liberdade de forma são variáveis aleatórias recozidas descritas por distribuições gaussianas. Esse modelo apresenta diagramas de fases diversos, sendo que é possível haver uma maior estabilidade das fases biaxiais em relação ao caso monodispersivo. No contexto de interações estéricas, Belli et al. [23] investigaram os efeitos da polidispersão de forma sobre o comportamento termodinâmico de um sistema de tabletas biaxiais. Nesse caso, os autores obtiveram vários diagramas nos quais as fases biaxiais podem ter regiões de estabilidade mais ampla e até mesmo a ocorrência de dois pontos de Landau.

Nesta tese iremos introduzir um sistema do tipo Maier-Saupe com o objetivo de entender como as variações de forma podem influenciar as fases nemáticas. Assim, no capítulo 3 apresentaremos um modelo de mistura binária de mesógenos intrinsecamente uniaxiais e intrinsecamente biaxiais. Seguiremos uma formulação mais apropriada à física de fluidos, de modo que os graus de liberdade de forma serão descritos por variáveis desordenadas recozidas. Não há dúvida de que uma mistura desse tipo estabelece uma conexão entre sistemas consituídos por mesógenos biaxiais e as misturas binárias de objetos uniaxiais. Apesar de envolver uma situação mais geral, o modelo permite fazer contato com a teoria de Landau-de Gennes sem muitas dificuldades. Além disso, mostraremos que essa mistura tem diagramas de fases muito ricos, vários comportamentos multicríticos e até mesmo reentrâncias associadas às estruturas biaxiais.

Certamente a forma dos mesógenos contribui para o comportamento termodinâmico 
de sistemas anisotrópicos. Porém, até agora, nossa discussão sobre mesofases nemáticas foi basicamente focada nos efeitos macroscópicos das simetrias das partículas. Todavia, é possível perceber que existe uma relação entre forma e simetria, o que não quer dizer que as duas são necessariamente equivalentes. Por exemplo, considere um elipsoide de revolução e um cilindro de tal maneira que ambos sejam prolatos e com dimensões características iguais. Não é dificil concluir que ambos têm as mesmas simetrias, embora apresentem medidas de format diferentes. A questão é que a dependência explícita com a forma pode influenciar bastante o diagrama de fases.

A forma das partículas desempenha um papel importante quando as interações são estéricas, ou seja, associadas a efeitos de volume excluído. Nesses casos, a temperatura não é uma grandeza relevante na termodinâmica de tais sistemas, que geralmente são denominados atérmicos. Assim, dentro de uma interpretação em termos de ensemble canônico, a única grandeza de interesse seria a concentração de partículas, ou, de forma equivalente, a fração volumétrica. Note que, no caso de mesofases líquido-cristalinas, podemos dizer que estamos trabalhando com materiais liotrópicos. Um ponto fundamental é que as transições que aparecem nos chamados sistemas de caroço duro são resultado de forças puramente entrópicas [24]. Num primeiro momento, não é óbvio que a entropia, por si só, possa levar a algum tipo de ordernamento, muito menos induzir transições de fases.

Sistemas com interações estéricas são fundamentais para o entendimento do comportamento macroscópico de materiais coloidais. Em particular, há vários resultados experimentais de interesse para coloides anisotrópicos. Por exemplo, van der Kooij e Lekkerkerker [25] observaram uma coexistência entre as fases nemática e isotrópica numa suspensão de partículas achatadas de gibbsita $\left(\mathrm{Al}(\mathrm{OH})_{3}\right)$. No caso de "bastões" de sílica $\left(\mathrm{O}_{2} \mathrm{Si}\right)$, Kuijk e colaboradores [26] encontraram várias fases de cristal líquido, variando a fração volumétrica e a relação de aspecto. Lemaire et al. [27] investigaram as propriedades magnéticas de uma suspensão aquosa de nanopartículas prolatas de goethita na presença de um campo magnético. De fato, suspensões coloidais de partículas minerais têm chamado muito a atenção para o estudo de mesofases líquido-cristalinas [28; 29].

Do ponto de vista teórico, Onsager [30] desenvolveu o primeiro modelo para um sistema

\footnotetext{
${ }^{1}$ Por exemplo, volume, área superficial ou raio de curvatura.
} 
de objetos anisotrópicos rígidos. Nesse modelo, a energia livre é dada por uma expansão virial truncada no termo associado ao volume excluído de pares. Mais do que isso, a energia livre é um funcional de uma distribuição que descreve a probabilidade de encontrar uma partícula com uma certa orientação. Em outras palavras, temos uma teoria do funcional densidade (DFT) para objetos anisotrópicos interagindo através de um potencial do tipo caroço duro [31]. Onsager aplicou essa teoria para estudar um sistema de bastões rígidos e mostrou que o modelo prevê uma transição nemático-isotrópica descontínua. Como todo o tratamento é desenvolvido no ensemble canônico, a transição se manisfesta na forma de uma coexistência de fases.

Contudo, a teoria de Onsager [30] é mais apropriada para descrever sistemas muito diluídos, o que certamente dificulta fazer comparações com os experimentos. Outro ponto delicado envolve as dimensões das partículas, que devem ser muito longas. De acordo com os trabalhos de Straley [32; 33], as partículas devem ter relação de aspecto maior que cem para que o modelo de Onsager seja válido quantitativamente. Note que partículas virais de TMV, mencionadas no trabalho de Onsager, têm relação de aspecto de aproximadamente 17. Não há dúvida de que é preciso haver modelos que possam descrever situações mais próximas da realidade.

De fato, muitas propostas, formuladas no contexto de DFT, têm sido desenvolvidas com o objetivo de ir além da teoria de Onsager. Muitas dessas teorias são bastante sofisticadas e certamente discutí-las nesta tese está além dos nossos propósitos. Sugerimos o trabalho de revisão de Mederos, Velasco e Martínez-Ratón [31], no qual várias abordagens de DFT são apresentadas em detalhes, muitas das quais formuladas para ir além das fases uniaxiais. No caso de estruturas nemáticas biaxiais em sistemas com interações estéricas, A. J. Masters desenvolve uma discussão sobre o tema no livro editado por Luckhurst e Sluckin [11.

Então, no capítulo 4 desta tese, iremos apresentar um cálculo do tipo DFT baseado nas aproximações de van der Waals. Ou seja, iremos fazer uma estimativa do volume livre acessível às partículas do sistema. Com isso, chegaremos em um funcional da energia livre que se reduz ao obtido por Onsager quando tomamos o limite de baixas densidades. Em seguida, aplicaremos a teoria para o caso de esferoides rígidos com dimensões finitas. Veremos que a distribuição orientacional terá algumas peculiaridades, de modo 
que o espaço orientacional poderá apresentar regiões proibidas. Por fim, calcularemos as equações de estado e mostraremos que o modelo exibe uma coexistência entre as fases nemática e isotrópica. Acreditamos que essa DFT, embora construída de maneira simples, seja apropriada para descrever o comportamento macroscópico de sistemas anisotrópicos densos. 


\section{Modelos microscópicos com}

\section{interações atrativas}

Neste capítulo discutiremos os modelos com interações atrativas utilizados na formulação estatística, de campo médio, das fases nemáticas uniaxiais e biaxiais. Apresentaremos uma discussão sobre interações de dispersão, também conhecidas genericamente como forças de van der Waals ou forças de London, e como essas interações estão relacionadas com a anisotropia molecular. Com base nessas interações, vamos introduzir uma versão de campo médio do modelo de Maier-Saupe para um sistema formado por objetos intrinsecamente biaxiais. Em seguida, iremos discutir um modelo mais geral para moléculas biaxiais bem como sua relação com o caso que envolve forças de dispersão.

\subsection{Interações de dispersão}

Nesta seção vamos revisar a discussão sobre forças intermoleculares na linha das expansões multipolares desenvolvidas no trabalho de Buckingham [34], mas com foco em moléculas neutras e apolares. Um tratamento semelhante, no contexto de cristais líquidos, é apresentado por Goossens [35] e van der Meer et al. [36]. Nesses trabalhos, os autores tentam obter energias de interações efetivas para descrever fases colestéricas, o que permitiria investigar a dependência da modulação com a temperatura. Basicamente, temos que levar em consideração as interações eletrostáticas entre as moléculas e os efeitos das flutuações quânticas. Seguiremos uma abordagem semelhante, mas nosso interesse é determinar um potencial efetivo de interação que possa levar a fases nemáticas uniaxiais e 
biaxiais.

\subsubsection{Tratamento geral da energia de dispersão}

Vamos considerar duas moléculas $A$ e $B$, cada uma formada por um conjunto de cargas pontuais $\left\{q_{X, i}\right\}$, com $X \in\{A, B\}$ e $i \in\{1,2,3, \cdots\}$, distribuídas no espaço de acordo com os vetores posição $\left\{\vec{r}_{X, i}\right\}$. A interação eletrostática entre $A$ e $B$ é descrita pelo operador hamiltoniano

$$
\mathcal{H}_{A B}=\sum_{i, j} \frac{q_{A, i} q_{B, j}}{\left|\vec{r}_{A, i}-\vec{r}_{B, j}\right|},
$$

associado ao acoplamento entre as duas moléculas. É possível obter uma expressão mais interessante supondo que $\vec{r}_{X, i}=\vec{r}_{X}+\vec{\rho}_{X, i}$, onde $\vec{r}_{X}$ é o vetor posição que localiza o centro de massa da molécula $X$, e $\vec{\rho}_{X, i}$ determina a posição relativa da carga $q_{X, i}$. Assim, podemos escrever a equação

$$
\left|\vec{r}_{A, i}-\vec{r}_{B, j}\right|=R\left[1-\frac{2 \vec{\rho}_{i j} \cdot \vec{u}}{R}+\frac{\left|\vec{\rho}_{i j}\right|^{2}}{R^{2}}\right]^{\frac{1}{2}}
$$

onde $\vec{R}=\vec{r}_{A}-\vec{r}_{B}$ é o vetor posição relativa entre $A$ e $B, \vec{\rho}_{i j}=\vec{\rho}_{B, j}-\vec{\rho}_{A, i}$ determina a posição relativa das cargas, e $\vec{u}$ é vetor unitário associado a $\vec{R}$. Optamos por simplificar a notação evitando o uso excessivo de índices, o que facilita o desenvolvimento dos cálculos. De posse da eq.(2.2), a energia de interação na eq.(2.1) pode ser reescrita utilizando uma expansão binomial. Obtemos assim

$$
\begin{aligned}
\mathcal{H}_{A B}= & \frac{1}{R} \sum_{i, j} q_{A, i} q_{B, j}+\frac{1}{R^{2}} \sum_{i, j} q_{A, i} q_{B, j} \vec{\rho}_{i j} \cdot \vec{u}+ \\
& +\frac{1}{2 R^{3}} \sum_{i, j} q_{A, i} q_{B, j}\left[3\left(\vec{\rho}_{i j} \cdot \vec{u}\right)^{2}-\left|\vec{\rho}_{i j}\right|^{2}\right]+\cdots,
\end{aligned}
$$

onde o primeiro termo é a interação monopolo-monopolo, o segundo indica o acoplamento monopolo-dipolo, o terceiro tem contribuições do tipo dipolo-dipolo e assim por diante. Nesse tratamento, a interação intermolecular pode ser entendida como uma expansão envolvendo as interações entre os vários momentos. Vamos supor que a carga total de cada molécula seja nula, pois estamos interessados nas situações mais simples de materiais líquido-cristalinos, cujos mesógenos são neutros em geral. Portanto, a primeira contribuição não trivial para o potencial de interação pode ser escrita na forma

$$
\mathcal{H}_{A B}=\frac{\vec{p}_{A} \cdot \vec{p}_{B}-3\left(\vec{p}_{A} \cdot \vec{u}\right)\left(\vec{p}_{B} \cdot \vec{u}\right)}{R^{3}}
$$


onde

$$
\vec{p}_{X}=\sum_{i} q_{X, i} \vec{\rho}_{X, i}
$$

é o momento de dipolo associado à molécula $X \in\{A, B\}$. O hamiltoniano de interação na eq. (2.4) é o ponto de partida das diversas formas de teorias de perturbação associadas às interações de van der Waals [37; 38] ou forças de dispersão de London [39]. De fato, as interações do tipo dipolo-dipolo são a base para o entendimento das forças intermoleculares atrativas em física da matéria condensada.

Nosso objetivo é obter uma expressão para a energia efetiva de interação entre $A$ e B. Assim, o hamiltoniano descrito pela eq. 2.4 será tratado como uma perturbação em relação ao sistema formado pelas duas moléculas isoladas, com hamiltoniano

$$
\mathcal{H}_{0}=\mathcal{H}_{A}+\mathcal{H}_{B}
$$

de tal modo que

$$
\mathcal{H}_{0}\left|n_{A} n_{B}\right\rangle=E_{n_{A} n_{B}}^{(0)}\left|n_{A} n_{B}\right\rangle
$$

onde cada autoestado do sistema é representado pelo vetor de estado $\left|n_{A} n_{B}\right\rangle$, com autoenergia dada por $E_{n_{A} n_{B}}^{(0)}=E_{n_{A}}^{(0)}+E_{n_{B}}^{(0)}$. Em particular, o estado fundamental será escrito na forma $|00\rangle$. Um ponto importante nos cálculos é que vamos supor que as moléculas não têm momento de dipolo permanente. Logo, a correção de primeira ordem na energia, dada por

$$
\varepsilon^{(1)}=\left\langle 00\left|\mathcal{H}_{A B}\right| 00\right\rangle,
$$

será identicamente nula, uma vez que a média do momento de dipolo de cada molécula é zero. Consequentemente, temos que levar em consideração termos de correção em segunda ordem,

$$
\varepsilon^{(2)}=-\sum_{n_{A}, n_{B}}^{\prime} \frac{\left|\left\langle 00\left|\mathcal{H}_{A B}\right| n_{A} n_{B}\right\rangle\right|^{2}}{\Delta E_{n_{A}}+\Delta E_{n_{B}}}
$$

onde

$$
\begin{aligned}
& \Delta E_{n_{A}}=E_{n_{A}}^{(0)}-E_{0}^{(0)}, \\
& \Delta E_{n_{B}}=E_{n_{B}}^{(0)}-E_{0}^{(0)}
\end{aligned}
$$

são as variações de energia entre os estados excitados e o estado fundamental para cada molécula. A soma percorre todos os estados excitados. 
Outra simplificação que adotaremos consiste em supor um espectro de energia de tal forma que possamos aproximar todas as diferenças $\Delta E_{n_{A}}$ e $\Delta E_{n_{B}}$ pelas suas médias, dadas por $\Delta E_{A}$ e $\Delta E_{B}$. Tal aproximação, utilizada em física atômica e molecular [39], é denominada closure approximation. Este tipo de aproximação é razoável quando $\Delta E_{n} \mathrm{e}$ $\Delta E_{n^{\prime}}$ não são muito diferentes, tipicamente, para todos os estados excitados. Antes de seguirmos com os cálculos, vamos reescrever a eq.2.4 numa forma mais compacta,

$$
H_{A B}=\frac{\vec{p}_{A} \cdot \mathbf{T} \cdot \vec{p}_{B}}{R^{3}}
$$

sendo que

$$
\mathbf{T}=\mathbf{1}-3 \vec{u} \otimes \vec{u}
$$

é um tensor clássico construído a partir do vetor $\vec{u}$ e 1 é o tensor identidade. Assim, a correção de segunda ordem na eq. 2.9. torna-se

$$
\varepsilon^{(2)}=-\frac{1}{\left(\Delta E_{A}+\Delta E_{B}\right) R^{6}} \sum_{n_{A}, n_{B}}^{\prime}\left|\left\langle 0\left|p_{A}^{\mu}\right| n_{A}\right\rangle T^{\mu \nu}\left\langle 0\left|p_{B}^{\nu}\right| n_{B}\right\rangle\right|^{2}
$$

onde $T^{\mu \nu}$ são as componentes de $\mathbf{T}$ e $p^{\mu}$ são as componentes do operador de dipolo $\vec{p}$. Também estamos fazendo uso da convenção de soma de Einstein para simplificar as expressões envolvendo os termos com contração de índices. Note que já aparece a dependência com a distância do tipo $R^{-6}$, típica de potenciais de interação associados às forças de van der Waals.

Para tornar a notação menos complicada, vamos escrever as médias das componentes de $\vec{p}$ como $\left\langle 0\left|p^{\mu}\right| n\right\rangle=p_{0 n}^{\mu}$, o que nos leva à equação

$$
\left|\left\langle 0\left|p_{A}^{\mu}\right| n_{A}\right\rangle T^{\mu \nu}\left\langle 0\left|p_{B}^{\nu}\right| n_{B}\right\rangle\right|^{2}=p_{0 n_{A}}^{\mu} p_{n_{A} 0}^{\gamma} T^{\mu \lambda} T^{\gamma \beta} p_{0 n_{B}}^{\lambda} p_{n_{B} 0}^{\beta}
$$

Um resultado interessante em física atômica e molecular é que as componentes do tensor de polarizabilidade molecular, dadas por $\alpha^{\mu \nu}$, estão relacionadas com os elementos de matriz $p_{0 n}^{\mu}$ através da expressão [38; 39]

$$
\begin{aligned}
\alpha^{\mu \nu} & =-2 \sum_{n}^{\prime} \frac{p_{0 n}^{\mu} p_{n 0}^{\nu}}{E_{0}-E_{n}}, \\
& \approx \frac{2}{\Delta E} \sum_{n}^{\prime} p_{0 n}^{\mu} p_{n 0}^{\nu},
\end{aligned}
$$


onde, na segunda linha, fazemos uso da closure approximation mais uma vez. Essa relação é importante pois nos permitirá associar a energia de interação com o tensor de polarizabilidade $\boldsymbol{\alpha}$.

Com o auxílio das eqs.2.14 2.15), é fácil ver que a soma na eq.2.13 pode ser escrita na forma

$$
\Delta E_{A} \Delta E_{B} \alpha_{A}^{\mu \gamma} T^{\mu \lambda} T^{\gamma \beta} \alpha_{B}^{\beta \lambda}
$$

É possível obter uma expressão mais interessante trabalhando com a convenção de soma e com as propriedades de simetria de $\boldsymbol{\alpha}$ e $\mathbf{T}$, uma vez que esses tensores são simétricos, por definição. Portanto, a correção de segunda ordem na eq.2.13 nos permite escrever a energia efetiva de interação na forma

$$
\varepsilon^{(2)} \approx-\frac{4 \Delta E_{A} \Delta E_{B}}{\left(\Delta E_{A}+\Delta E_{B}\right) R^{6}} \operatorname{Tr}\left(\mathbf{T} \boldsymbol{\alpha}_{A} \mathbf{T} \boldsymbol{\alpha}_{B}\right)
$$

onde Tr denota o traço, calculado pela soma dos elementos da diagonal principal.

Grosso modo, podemos dizer que a energia efetiva de interação decai com a sexta potência da distância (forças de van der Waals), dependendo das polarizabilidades e da orientação do vetor que descreve a posição relativa das moléculas, como esquematizado na figura 2.1. Levando em consideração apenas a energia de dispersão, Buckingham [34] obtém uma equação do mesmo tipo considerando interações eletrostáticas e aplicando o formalismo da teoria de perturbação estacionária. Uma expressão semelhante também é usada por Palffy-Muhoray e Zheng [40], no contexto de campo médio, para estudar fases biaxiais num modelo molecular onde os mesógenos interagem através de forças de van der Waals.

Como já havíamos mencionado, nosso interesse é obter um potencial de interação efetivo que seja apropriado para descrever fases nemáticas uniaxiais e biaxiais. Essas fases não têm ordem translacional de longo alcance, pois os centros de massa (ou de carga) das moléculas estão distribuídos isotropicamente no espaço. Logo, é razoável supor que a interação de pares dependa da distância entre os mesógenos, mas não dependa da orientação do vetor posição relativa $\vec{R}$. Isso sugere que podemos tomar a média da eq. 2.17) sobre todas as orientações descritas por $\vec{u}$. Logo, temos que determinar a média de $\mathbf{T}$, que será dada por um tensor isotrópico, com componentes expressas em termos de combinações lineares de tensores de Kronecker. Portanto, vamos definir a interação 


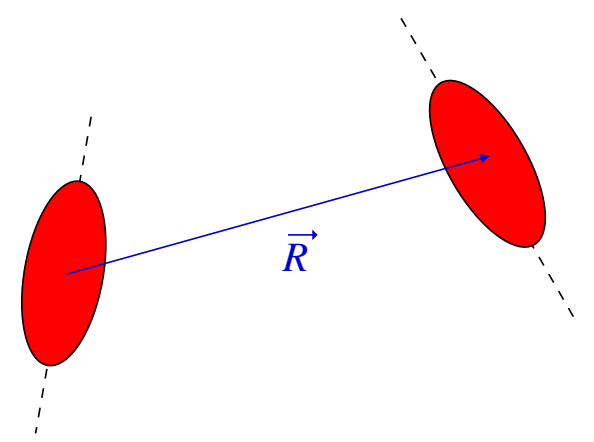

Figura 2.1: A interação efetiva depende da orientação relativa dos mesógenos e do vetor posição relativa $\vec{R}$ que conectada os centros de massa.

efetiva intermolecular,

$$
\mathcal{H}_{e f f}=-\frac{\epsilon}{R^{6}} \operatorname{Tr}\left(\boldsymbol{\alpha}_{A} \boldsymbol{\alpha}_{B}\right)
$$

onde $\epsilon$ é uma constante positiva. Esse resultado, embora simples, mostra como as propriedades orientacionais das moléculas, decodificadas em $\boldsymbol{\alpha}$, influenciam as interações. Uma observação importante é que o tensor de polarizabilidade também depende da forma molecular 1

No caso mais simples de moléculas com simetria esférica, a eq.2.16 já leva ao resultado conhecido como fórmula de London [39],

$$
\varepsilon^{(2)} \propto-\frac{\Delta E_{A} \Delta E_{B}}{\left(\Delta E_{A}+\Delta E_{B}\right)} \frac{\bar{\alpha}_{A} \bar{\alpha}_{B}}{R^{6}},
$$

onde $\bar{\alpha}$ é simplesmente a polarizabilidade molecular, uma vez que $\boldsymbol{\alpha}$ é proporcional ao tensor identidade. Para moléculas anisotrópicas num fluido isotrópico, podemos definir $\bar{\alpha}$ como a polarizabilidade média, dada pela média aritmética dos autovalores de $\boldsymbol{\alpha}$. De fato, podemos decompor o tensor de polarizabilidade numa parte isotrópica,

$$
\overline{\boldsymbol{\alpha}} \equiv \bar{\alpha} \mathbf{1}=\frac{\alpha^{\mu \mu}}{3} \mathbf{1}
$$

e numa parte anisotrópica

$$
\boldsymbol{\alpha}_{a} \equiv \boldsymbol{\alpha}-\bar{\alpha} \mathbf{1}
$$

que é um tensor simétrico de traço nulo. Geralmente, a parte isotrópica $\overline{\boldsymbol{\alpha}}$ é deixada de lado num estudo de transições envolvendo sistemas líquido-cristalinos. Além disso, devido à ausência de ordem posicional de longo alcance, também não se considera a dependência

\footnotetext{
${ }^{1}$ Note que $\boldsymbol{\alpha}$ tem dimensão de volume.
} 


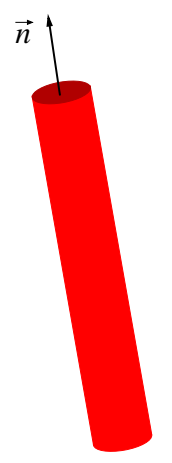

Figura 2.2: Representação esquemática de uma molécula intrinsecamente uniaxial que tem a forma efetiva de um cilindro prolato. O estado orientacial é determinado pelo diretor local $\vec{n}$.

explícita com a distância, o que simplifica bastante os cálculos. Portanto, a energia de interação é simplesmente dada por

$$
-\epsilon \operatorname{Tr}\left(\boldsymbol{\alpha}_{a, A} \boldsymbol{\alpha}_{a, B}\right)
$$

o que evidencia o papel relevante dos efeitos da anisotropia molecular sobre o comportamento termodinâmico das fases líquido-cristalinas.

\subsubsection{Moléculas uniaxiais}

Um caso especial muito interessante é o modelo de Maier-Saupe para moléculas com simetria cilíndrica. Nesse modelo, podemos imaginar que os mesógenos são descritos, por exemplo, através de objetos com formas efetivas de cilindros circulares retos, como mostrado na figura 2.2. Outra opção seria considerar elipsoides de revolução, também chamados de esferoides.

Vamos supor, por simplicidade, que as moléculas são prolatas com polarizabilidade $\alpha_{\|}$ao longo do eixo de simetria, dado pelo vetor unitário $\vec{n}$, e polarizabilidade $\alpha_{\perp}$ nas direções perpendiculares a $\vec{n}$. Assim, a parte anisotrópica de $\boldsymbol{\alpha}$ será dada por

$$
\boldsymbol{\alpha}_{a}=\left(\alpha_{\|}-\alpha_{\perp}\right) \mathbf{q},
$$

onde

$$
\mathbf{q}=\vec{n} \otimes \vec{n}-\frac{1}{3} \mathbf{1},
$$

é um tensor simétrico de traço nulo que especifica o estado orientacional da molécula, 
cujas componentes são dadas por

$$
q^{\mu \nu}=n^{\mu} n^{\nu}-\frac{\delta^{\mu \nu}}{3}
$$

onde $\mu, \nu=x, y, z$, tomadas a partir de um sistema de referência fixo no laboratório. Usando uma representação esférica para $\vec{n}$,

$$
\begin{aligned}
& n_{x}=\sin \theta \cos \phi, \\
& n_{y}=\sin \theta \sin \phi, \\
& n_{z}=\cos \theta,
\end{aligned}
$$

$\operatorname{com} \phi \in[0,2 \pi]$ e $\theta \in[0, \pi]$, é fácil mostrar que o tensor q é dado pela matriz

$$
\left[\begin{array}{ccc}
\sin ^{2} \theta \cos ^{2} \phi-\frac{1}{3} & \frac{1}{2} \sin ^{2} \theta \sin 2 \phi & \frac{1}{2} \sin ^{2} \theta \cos 2 \phi \\
\frac{1}{2} \sin ^{2} \theta \sin 2 \phi & \sin ^{2} \theta \sin ^{2} \phi-\frac{1}{3} & \frac{1}{2} \sin ^{2} \theta \sin 2 \phi \\
\frac{1}{2} \sin ^{2} \theta \cos 2 \phi & \frac{1}{2} \sin ^{2} \theta \sin 2 \phi & \cos ^{2} \theta-\frac{1}{3}
\end{array}\right] .
$$

Obviamente, as orientações moleculares são descritas por um conjunto contínuo de estados parametrizados pelos ângulos polar $\theta$ e azimutal $\phi$.

Certamente, é possível fazer o estudo do sistema levando em consideração estados orientacionais de acordo com eq.(2.26). Contudo, existe uma simplificação interessante proposta por Zwanzig [41, que consiste em supor que as orientações moleculares são restritas aos eixos do sistema de referência do laboratório. Logo, no caso de moléculas com simetria cilíndrica, $\mathbf{q}$ possui apenas três estados dados por

$$
\left\{\left[\begin{array}{lll}
-\frac{1}{3} & & \\
& -\frac{1}{3} & \\
& & \frac{2}{3}
\end{array}\right],\left[\begin{array}{lll}
-\frac{1}{3} & & \\
& \frac{2}{3} & \\
& & -\frac{1}{3}
\end{array}\right],\left[\begin{array}{lll}
\frac{2}{3} & & \\
& -\frac{1}{3} & \\
& & -\frac{1}{3}
\end{array}\right]\right\} \text {. }
$$

Essa discretização é muito útil, uma vez que permite trabalhar com expressões mais tratáveis, sem levar a perdas qualitativas significativas, o que descaracterizariam bastante o problema. De fato, Zwanzig [41] adotou esse esquema para estudar um gás de partículas prolatas rígidas, calculando até o sétimo coeficiente virial. Num trabalho anterior, Zwanzig considera um gás de cubos rígidos, bem como um sistema de placas quadradas rígidas, com restrições orientacionais também visando o cálculo de coeficientes viriais [42]. 
Tendo descrito os estados orientacionais das moléculas, é possível introduzir o modelo de Maier-Saupe a partir de um potencial de pares da forma

$$
\begin{aligned}
\mathcal{H}_{M S} & =-\epsilon\left(\alpha_{\|}-\alpha_{\perp}\right)^{2} \mathbf{q}_{A} \cdot \mathbf{q}_{B}, \\
& =-U_{0} \mathbf{q}_{A} \cdot \mathbf{q}_{B},
\end{aligned}
$$

com $U_{0}>0$. Por simplicidade, vamos escrever $\mathbf{q}_{A} \cdot \mathbf{q}_{B} \equiv \operatorname{Tr}\left(\mathbf{q}_{A} \mathbf{q}_{B}\right)$. Também estamos assumindo que as moléculas são idênticas. Basicamente, a teoria molecular de MaierSaupe considera que as fases nemáticas uniaxiais estão relacionadas com a anisotropia de forma das moléculas. Esse modelo apresenta uma transição descontínua entre as fases nemática e isotrópica à medida que a temperatura aumenta.

\subsection{Interações de dispersão em moléculas intrinseca- mente biaxiais}

No caso de moléculas intrinsecamente biaxiais, a polarizabilidade molecular tem três autovalores distintos, $\alpha_{i}$, cada um associado a um autovetor $\vec{n}_{i}$, com $i=1,2,3$, de tal modo que

$$
\boldsymbol{\alpha}=\sum_{i} \alpha_{i} \vec{n}_{i} \otimes \vec{n}_{i}
$$

Um dos exemplos mais simples é o de moléculas com forma efetiva de paralelepípedo retângulo, como mostrado na figura 2.3. Elipsoides triaxiais seriam uma possibilidade interessante para os mesógenos, uma vez que tais objetos têm propriedades de simetria consistentes com (2.29). Todavia, é importante mencionar que moléculas com formas mais complicadas podem levar a mesofases biaxiais.

Como já discutido anteriormente, nosso interesse consiste em determinar a parte anisotrópica de $\boldsymbol{\alpha}$,

$$
\boldsymbol{\alpha}_{a}=\sum_{i}\left(\alpha_{i}-\bar{\alpha}\right) \vec{n}_{i} \otimes \vec{n}_{i}
$$

Porém, devido à biaxialidade intrínseca dos mesógenos, é conveniente escrever $\boldsymbol{\alpha}_{a}$ em termos de duas componentes, cada uma sendo um tensor simétrico com traço nulo, como mostrado no apêndice A. Ou seja, a menos de uma constante multiplicativa, podemos escrever $\boldsymbol{\alpha}_{a}$ como

$$
\boldsymbol{\alpha}_{a}=3 \mathbf{q}+\Delta \mathbf{b}
$$




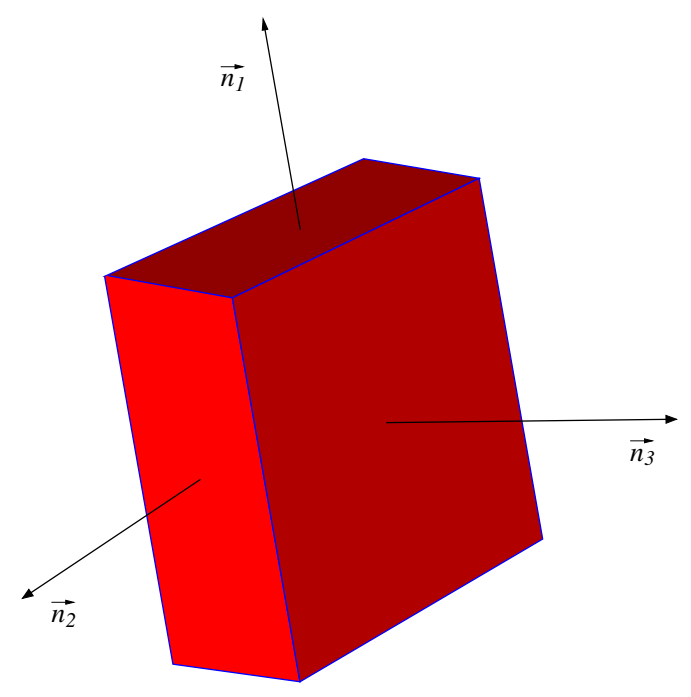

Figura 2.3: Representação esquemática de uma molécula intrinsecamente biaxial. O estado orientacial da molécula é especificado pelo sistema de referência local $\vec{n}_{1}, \vec{n}_{2}$ e $\vec{n}_{3}$.

onde

$$
\begin{aligned}
& \mathbf{q}=\vec{n}_{1} \otimes \vec{n}_{1}-\frac{1}{3} \mathbf{1}, \\
& \mathbf{b}=\vec{n}_{2} \otimes \vec{n}_{2}-\vec{n}_{3} \otimes \vec{n}_{3},
\end{aligned}
$$

são tensores construídos a partir dos autovetores de $\boldsymbol{\alpha}$ de tal modo que satisfaçam as simetrias das moléculas, e $\Delta$ é uma parâmetro escalar associado com o grau de biaxialidade dos mesógenos. No caso das eqs. 2.31), estamos supondo que $\alpha_{1}$ é o autovalor de maior módulo.

Não é difícil mostrar a relação entre o grau de biaxialidade e os autovalores do tensor de polarizabilidade. Também fica claro que, no caso de moléculas com simetria cilíndrica, os dois autovalores de menor módulo são iguais, o que significa que não deve haver contribuições de b. Note que podemos adotar formas diferentes de escrever $\boldsymbol{\alpha}_{a}$, desde que se preserve a simetria "quadrupolar". Por simplicidade, vamos assumir que o estado orientacional molecular será dado pelo tensor $\Omega \equiv \boldsymbol{\alpha}_{a}$. Uma vez que definimos o estado orientacional de um mesógeno, podemos passar adiante e introduzir um modelo de campo médio apropriado para estudar fases nemáticas uniaxiais e biaxiais.

Considere um sistema formado por objetos intrinsecamente biaxiais. Vamos supor uma energia de interação de pares que dependa apenas das orientações relativas dos objetos. 
Assim, o modelo de campo médio será dado pelo hamiltoniano

$$
\mathcal{H}=-\frac{\epsilon}{2 N} \sum_{i, j=1}^{N} \boldsymbol{\Omega}_{i} \cdot \boldsymbol{\Omega}_{j}
$$

onde $\epsilon$ é uma constante de interação entre os tensores de quadrupolo $\Omega_{i}$ e $\Omega_{j}$, definidos sobre os sítios $i$ e $j$ de uma rede. É importante lembrar que o hamiltoniano na eq. 2.32 simplesmente descreve um sistema de moléculas não-esféricas interagindo através de forças de dispersão de London, como já discutido nas seções anteriores. Embora nosso foco sejam mesofases líquido-cristalinas, um modelo de interações quadrupolares semelhante foi estudado por Boccara et al. [43], que investigaram o diagrama de fases de um sistema formado por elipsoides assimétricos.

Vale a pena mencionar que as componentes do tensor de quadrupolo são funções dos cossenos diretores que conectam o sistema de referência fixo no objeto com o sistema de referência do laboratório. A maneira habitual de relacionar os dois sistemas de referência é considerar que os eixos principais de um objeto estão associados com os eixos do laboratório através de uma rotação parametrizada pelos ângulos de Euler [44]. Certamente, isso levaria o tensor de quadrupolo a exibir uma representação matricial bem complicada.

Contudo, é posível simplificar a análise seguindo a proposta de estados discretos de Zwanzig [41], segundo a qual os objetos se orientam apenas nas direções dos eixos cartesianos (figura 2.4. Consequentemente, $\boldsymbol{\Omega}$ assume somente seis estados possíveis, representados pelas matrizes

$$
\begin{aligned}
& {\left[\begin{array}{rrr}
-1+\Delta & & \\
& -1-\Delta & \\
& & 2
\end{array}\right],\left[\begin{array}{lll}
-1-\Delta & \\
& -1+\Delta & \\
& & 2
\end{array}\right]} \\
& {\left[\begin{array}{ccc}
-1+\Delta & \\
& 2 & \\
& & -1-\Delta
\end{array}\right],\left[\begin{array}{lll}
2 & & \\
& -1+\Delta & \\
& & -1-\Delta
\end{array}\right],} \\
& {\left[\begin{array}{ccc}
-1-\Delta & \\
& 2 & \\
& & -1+\Delta
\end{array}\right],\left[\begin{array}{lll}
2 & & \\
& -1-\Delta & \\
& & -1+\Delta
\end{array}\right] \text {. }}
\end{aligned}
$$




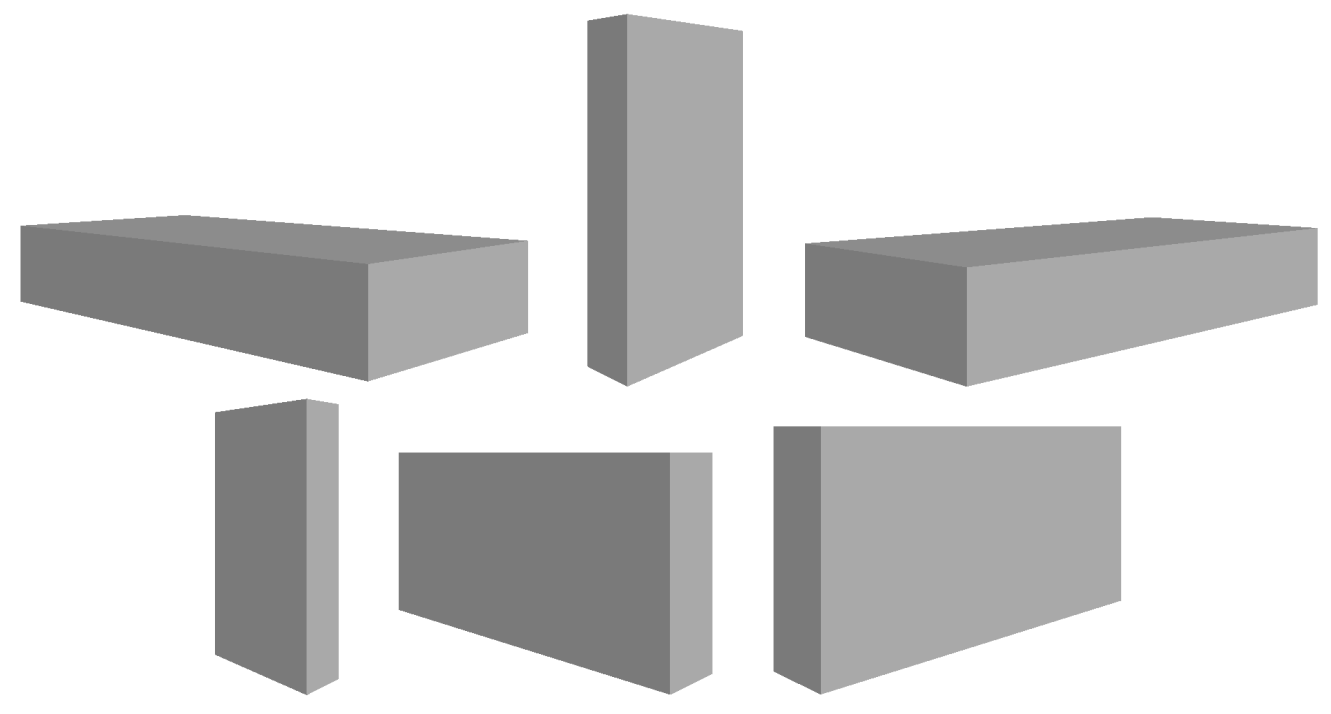

Figura 2.4: Discretização dos estados orientacionais para um objeto intrinsecamente biaxial.

Dessa forma, o problema fica reduzido ao estudo das propriedades termodinâmicas de um modelo efetivo de seis estados. No caso particular de moléculas com simetria cilíndrica2 o sistema pode ser mapeado num modelo de Potts de três estados.

Uma vez tendo o hamiltoniano do sistema, dado por eq.2.32, podemos seguir para a estatística e termodinâmica. De fato, após algumas manipulações algébricas, é possível escrever a função de partição na forma

$$
Z=\sum_{\left\{\boldsymbol{\Omega}_{i}\right\}} \exp \left[\frac{\beta \epsilon}{2 N} \sum_{\mu=1}^{3}\left(\sum_{i=1}^{N} \Omega_{i}^{\mu \mu}\right)^{2}\right]
$$

onde $\beta \epsilon=1 / T$, sendo que $T$ é a temperatura reduzida. Essa expressão é mais apropriada para o uso de representações integrais no contexto de campo médio, uma vez que a soma no argumento da exponencial na eq. 2.34 pode ser fatorizada com o auxílio de identidades gaussianas do tipo

$$
\exp \left(a_{\mu}^{2}\right)=\frac{1}{\sqrt{\pi}} \int_{-\infty}^{+\infty} d x_{\mu} \exp \left(-x_{\mu}^{2}+2 a_{\mu} x_{\mu}\right)
$$

o que nos permite escrever

$$
Z=\frac{1}{\pi^{3 / 2}}\left(\prod_{\mu} \int d x_{\mu}\right) \sum_{\left\{\boldsymbol{\Omega}_{i}\right\}} \exp \left[-\sum_{\mu=1}^{3} x_{\mu}^{2}+\sqrt{\frac{2 \beta \epsilon}{N}} \sum_{\mu=1}^{3} x_{\mu} \sum_{i} \Omega_{i}^{\mu \mu}\right] .
$$

\footnotetext{
${ }^{2}$ É fácil ver que nesses casos devemos ter $\Delta \in\{0,3\}$.
} 
É interessante fazer uma mudança da variáveis do tipo

$$
x_{\mu}^{2}=\frac{N \beta \epsilon}{2} q_{\mu}^{2}
$$

que nos leva à equação

$$
Z=\left(\frac{N \beta \epsilon}{2 \pi}\right)^{3 / 2}\left(\prod_{\mu} \int d q_{\mu}\right) \sum_{\left\{\boldsymbol{\Omega}_{i}\right\}} \exp \left[-\frac{N \beta \epsilon}{2} \sum_{\mu=1}^{3} q_{\mu}^{2}+\beta \epsilon \sum_{\mu=1}^{3} q_{\mu} \sum_{i} \Omega_{i}^{\mu \mu}\right] .
$$

Temos então apenas termos lineares nos momentos de quadrupolo $\boldsymbol{\Omega}_{i}$, o que facilita o cálculo da soma sobre todas as orientações moleculares. Portanto, a menos de constantes irrelevantes, a função de partição assume a forma integral

$$
Z=\int d \mathbf{q} \exp (-N \beta \epsilon \phi)
$$

onde

$$
\begin{aligned}
\phi= & \frac{1}{2}\left(q_{1}^{2}+q_{2}^{2}+q_{3}^{2}\right)+\left(q_{1}+q_{2}+q_{3}\right)-T \ln 2 \\
& -T \ln \left\{\exp \left(\frac{3 q_{1}}{T}\right) \cosh \left[\frac{\left(q_{2}-q_{3}\right) \Delta}{T}\right]\right. \\
& +\exp \left(\frac{3 q_{2}}{T}\right) \cosh \left[\frac{\left(q_{3}-q_{1}\right) \Delta}{T}\right] \\
& \left.+\exp \left(\frac{3 q_{3}}{T}\right) \cosh \left[\frac{\left(q_{1}-q_{2}\right) \Delta}{T}\right]\right\}
\end{aligned}
$$

é o chamado funcional da energia livre do sistema. A conexão com a termodinâmica é feita tomando o limite termodinâmico $(N \rightarrow \infty)$ e aplicando o método da integração assintótica de Laplace. Como resultado, os estados termodinâmicos estáveis são caracterizados por $\left(q_{1}, q_{2}, q_{3}\right)$ que minimizam o funcional $\phi$.

Os pontos extremos de $\phi$ são aqueles para os quais $\partial \phi / \partial q_{i}=0$, com $i=1,2,3$. Essa condição leva a um sistema de equações não-lineares para o qual $q_{1}+q_{2}+q_{3}=0$, que satisfaz a condição de traço do problema. Essa condição sugere que podemos fazer uma mudança de variáveis do tipo

$$
\begin{aligned}
& q_{1}=-\frac{S+\eta}{2}, \\
& q_{2}=-\frac{S-\eta}{2}, \\
& q_{3}=S
\end{aligned}
$$




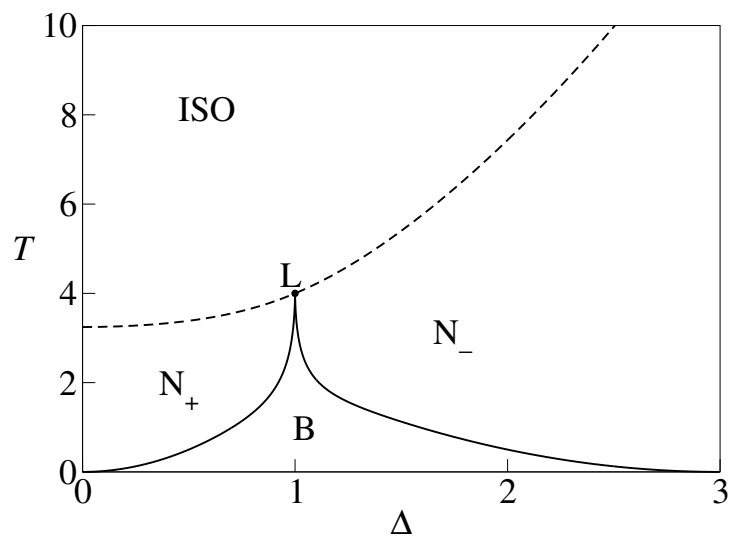

Figura 2.5: Diagrama de fases do modelo Maier-Saupe discretizado, em termos da temperatura reduzida $T$ e da biaxialidade $\Delta$. ISO: isotrópica. $\mathrm{N}_{+}$: uniaxial calamítica. $\mathrm{N}_{+}$: uniaxial discótica. B: biaxial. L: ponto de Landau.

Novamente, calculando os extremos de $\phi=\phi(S, \eta)$, chegamos a um sistema de equações do tipo $S=f_{1}(S, \eta)$ e $\eta=f_{2}(S, \eta)$. Em geral, esse sistema admite mais de uma solução. Contudo, estamos interessados apenas na solução para a qual $\phi$ é mínimo, fixadas a temperatura reduzida $T$ e a biaxialidade $\Delta$ dos objetos que formam o sistema.

A figura 2.5 exibe o diagrama de fases do modelo Maier-Saupe discretizado para objetos intrinsecamente biaxiais. Podemos observar quatro fases: isotrópica (ISO), nemática uniaxial calamítica $\left(\mathrm{N}_{+}\right)$, nemática uniaxial discótica $\left(\mathrm{N}_{-}\right)$e nemática biaxial $(\mathrm{B})$. As fases nemáticas uniaxiais e isotrópica são separadas por uma linha de transição de primeira ordem (linha tracejada). As fases nemáticas uniaxiais e biaxial são delimitadas por linhas de transição de segunda ordem (linhas contínuas). As linhas de primeira ordem encontram as linhas de segunda ordem num ponto multicrítico denominado ponto de Landau, L. O diagrama da figura 2.5 possui a mesma topologia do diagrama obtido por Boccara et al. [43], que consideram graus de liberdade orientacionais contínuos. Isso sugere que a discretização que adotamos com as eqs. 2.33 não modifica qualitativamente a física do problema no nível de campo médio. De fato, em ambos os casos, os estados orientacionais são caracterizados por tensores de quadrupolo. Ainda no contexto de campo médio, Sauerwein e de Oliveira [45] estudaram um modelo de rede para mesógenos biaxiais com orientações discretas, cujo diagrama de fases é qualitativamente consistente com o que obtivemos.

O diagrama na figura 2.5 pode ser interpretado em termos da análise dos níveis de 
energia do modelo como função do parâmetro de biaxialidade, como mostrado na figura 2.6. De um modelo geral, os níveis de energia têm graus de degenerescência variados, sendo que alguns desses níveis se cruzam para certos valores de $\Delta$. Como essas degenerescências contribuem para a parte entrópica da energia livre, é razoável verificar a possibilidade de mudanças qualitativas no diagrama de fases nas vizinhanças dos cruzamentos. De fato, a partir da figura 2.6, vemos que alguns níveis se encontram para $\Delta=0,1,3$. Nos casos de $\Delta=0$ e $\Delta=3$, os mesógenos possuem, respectivamente, simetria cilíndrica prolata (tipo bastão) e oblata (tipo disco), como pode ser verificado através das eqs. 2.33). O caso especial $\Delta=1$ corresponde ao ponto multicrítico de Landau. Um fato interessante é que é possível estabelecer um mapeamento entre as temperaturas de transição de cada lado do ponto de Landau através das expressõe: $\mathrm{s}^{3}$

$$
\Delta \rightarrow \frac{3-\Delta}{1+\Delta} \quad \text { e } \quad \epsilon \rightarrow \frac{4 \epsilon}{(1+\Delta)^{2}}
$$

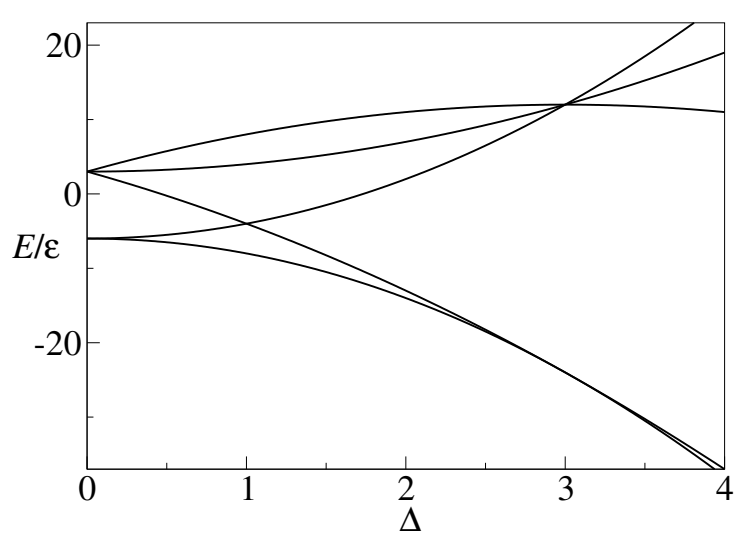

Figura 2.6: Níveis de energia do modelo Maier-Saupe discretizado para moléculas intrinsecamente biaxiais.

\subsubsection{Conexão com a teoria de Landau-de Gennes}

Vamos agora estabelecer contato com o modelo de dispersão para mesógenos biaxiais e a teoria fenomenológica de Landau-de Gennes [1; 4; 46]. É importante lembrar que esse contato é relevante do ponto de vista experimental, pois, partindo de um modelo microscópico, podemos relacionar os coeficientes da expansão de Landau com os parâmetro

\footnotetext{
${ }^{3}$ Boccara et al. 43 também mostram um mapeamento semelhante.
} 
microscópicos do modelo. Primeiramente, devemos introduzir um parâmetro de ordem que, no nosso caso, é um tensor simétrico de traço nulo do tipo

$$
\mathbf{Q}=\left[\begin{array}{lll}
q_{1} & & \\
& q_{2} & \\
& & q_{3}
\end{array}\right] \text {. }
$$

A partir desse tensor, é necessário definir os invariantes do parâmetro de ordem, que são dados apenas por dois invariantes fundamentais,

$$
I_{2}=\operatorname{Tr}^{2} \quad \text { e } \quad I_{3}=\operatorname{Tr} \mathbf{Q}^{3}
$$

De posse dos invariantes do parâmetro de ordem, devemos escrever uma expansão do funcional $\phi$ em termos de $I_{2}$ e $I_{3}$. Devido à simetria e à estabilidade, a expansão é feita com termos até a sexta ordem [46], o que leva a

$$
\phi=\phi_{0}+\frac{A}{2} I_{2}+\frac{B}{3} I_{3}+\frac{C}{4} I_{2}^{2}+\frac{D}{5} I_{2} I_{3}+\frac{E}{6} I_{2}^{3}+\frac{E^{\prime}}{6} I_{3}^{2}+\cdots,
$$

onde $\phi_{0}$ é a energia livre da fase isotrópica. A partir da eq. 2.39), é possível mostrar que os coeficientes da expansão são dados por

$$
\begin{aligned}
A & =1-\frac{3}{T}-\frac{\Delta^{2}}{T}, & B & =-\frac{9\left(1-\Delta^{2}\right)}{2 T^{2}}, \\
C & =\frac{1}{16}, & D & =0, \\
E & =-\frac{41}{7680} & E^{\prime} & =\frac{1}{640}>0 .
\end{aligned}
$$

Esses coeficientes dependem da temperatura reduzida $T$ e do parâmetro de biaxialidade $\Delta$. As coordenadas do ponto de Landau 4 são obtidas quando

$$
A=0 \quad \text { e } \quad B=0,
$$

o que resulta em

$$
\Delta_{L}^{2}=1 \quad \text { e } \quad T_{L}=4
$$

Um fato importante é que o ponto de Landau é estável, pois o coeficiente do termo de quarta ordem é positivo $(C>0)$. Além disso, o coeficiente $E^{\prime}$ também é positivo, o que garante a estabilidade da fase biaxial nas vizinhanças do ponto de Landau [4; 46].

\footnotetext{
${ }^{4}$ Assim como no trabalho de Gramsbergen et al. [46], estamos assumindo que essas condições definem o ponto de Landau.
} 


\subsection{Modelos de Straley e SVD}

Começamos o capítulo discutindo a origem micróscopica das interações de dispersão e como utilizá-las para obter um potencial efetivo de pares que pudesse levar a fases nemáticas biaxiais. No entanto, é possível obter uma energia de interação mais geral que, inclusive, tem como caso particular o modelo baseado em forças de dispersão, como apresentado na seção 2.2. Nesta seção vamos apresentar um modelo bastante geral para nemáticos biaxiais e analisar os casos específicos de interesse.

\subsubsection{Modelo de Straley}

Esse modelo foi introduzido por Straley [15], que escreveu um potencial de pares:

- quadrático nos cossenos diretores que descrevem as orientações relativas das moléculas;

- invariante pela inversão dos eixos (head-tail symmetry);

- simétrico com respeito à troca das partículas.

Vamos supor que os eixos principais das moléculas sejam dados por $\left\{\vec{n}_{1}, \vec{n}_{2}, \vec{n}_{3}\right\}$ e $\left\{\vec{n}_{1}^{\prime}, \vec{n}_{2}^{\prime}, \vec{n}_{3}^{\prime}\right\}$, respectivamente. Assumindo as hipóteses de Straley, o potencial de interação pode ser escrito na forma

$$
\begin{aligned}
V= & a_{1}+\frac{a_{2}}{2}\left[\left(\vec{n}_{1} \cdot \vec{n}_{1}^{\prime}\right)^{2}-1\right]+2 a_{3}\left[\left(\vec{n}_{3} \cdot \vec{n}_{3}^{\prime}\right)^{2}-\left(\vec{n}_{2} \cdot \vec{n}_{2}^{\prime}\right)^{2}\right]+ \\
& +\frac{a_{4}}{2}\left[\left(\vec{n}_{2} \cdot \vec{n}_{2}^{\prime}\right)^{2}-\left(\vec{n}_{2} \cdot \vec{n}_{3}^{\prime}\right)^{2}-\left(\vec{n}_{3} \cdot \vec{n}_{2}^{\prime}\right)^{2}+\left(\vec{n}_{3} \cdot \vec{n}_{3}^{\prime}\right)^{2}\right]
\end{aligned}
$$

onde $a_{i}(i=1,2,3,4)$ são coeficientes que podem ser funções da distância entre as moléculas. Por simplicidade, vamos considerar que tais coeficientes sejam constantes, pois estamos interessados em fases que não têm ordenamento espacial de longo alcance. Assim, Straley propõe reescrever a eq. 2.48 como

$$
V=a_{1}+a_{2} F_{1}(\theta)+a_{3}\left[F_{2}(\theta, \phi)+F_{3}(\theta, \psi)\right]+a_{4} F_{4}(\theta, \phi, \psi),
$$

sendo que $F_{i}(i=1,2,3,4)$ são funções básicas dos ângulos de Euler $(\theta, \phi, \psi)$. Essas funções seriam construídas de tal modo a satisfazer as simetrias das moléculas. No caso particular de objetos com a forma de um paralelepípedo retangular, como mostrado na 
figura 2.3, as funções devem ser invariantes pelas transformações

$$
\begin{aligned}
& \phi \rightarrow \phi+\pi \\
& \psi \rightarrow \psi+\pi \\
& \theta \rightarrow \pi-\theta, \quad \phi \rightarrow \pi-\phi, \quad \psi \rightarrow-\psi
\end{aligned}
$$

Uma maneira natural de encontrar $F_{i}$ seria utilizar um conjunto completo de funções ortogonais definidas em termos dos ângulos de Euler5. Dessa forma, Straley [15] chega a um conjuto de funções básicas dadas por

$$
\begin{aligned}
& F_{1}=\frac{1}{2}\left(3 \cos ^{2} \theta-1\right), \\
& F_{2}=\sin ^{2} \theta \cos 2 \phi, \\
& F_{3}=\sin ^{2} \theta \cos 2 \psi, \\
& F_{4}=\frac{1}{2}\left(1+\cos ^{2} \theta\right) \cos 2 \phi \cos 2 \psi-\cos \theta \sin 2 \phi \sin 2 \psi .
\end{aligned}
$$

Vale a pena lembrar que $(\theta, \phi, \psi)$ especificam a orientação relativa dos mesógenos.

Straley estudou o modelo dado pela eq.2.48 fazendo uma associação com um gás de "paralelepípedos rígidos", relacionando o potencial de interação com o volume excluído de pares. Isso permitiu escrever os coeficientes $a_{i}$ em termos dos parâmetros de forma das moléculas. Um ponto importante é que Straley se baseou na ideia de que as fases biaxiais estariam relacionadas a algum tipo de competição entre as fases uniaxiais calamíticas e discóticas. Dentro dessa premissa, é possível determinar um conjunto de parâmetros que leva a esse tipo de comportamento. Assim, seguindo um tratamento de campo médio, Straley mostrou que o modelo possui um diagrama de fases parecido com o da figura 2.5 . com uma fase biaxial entre duas fases uniaxiais e um ponto de Landau.

\subsubsection{Modelo SVD}

Décadas depois do trabalho de Straley [15], Sonnet, Virga e Durand [16] retomaram a energia de interação na eq. 2.48) e perceberam que ela poderia ser reescrita numa forma mais compacta com a ajuda dos tensores $\mathbf{q}$ e $\mathbf{b}$ definidos pelas eqs.2.31. Como resultado, o estado orientacional de uma molécula seria especificado por um par de tensores de

\footnotetext{
${ }^{5}$ Basicamente, as matrizes de rotação de Wigner.
} 
quadrupolo $(\mathbf{q}, \mathbf{b})$, e o potencial de pares na eq. 2.48) assumiria a forma

$$
V=-U_{0}\left[\mathbf{q} \cdot \mathbf{q}^{\prime}+\gamma\left(\mathbf{q} \cdot \mathbf{b}^{\prime}+\mathbf{b} \cdot \mathbf{q}^{\prime}\right)+\lambda \mathbf{b} \cdot \mathbf{b}^{\prime}\right]
$$

onde $U_{0}$ seria uma constante positiva, e os parâmetros $\gamma$ e $\lambda$ estariam associados à simetria e à forma dos mesógenos. Vamos denotar a energia de interação na eq. 2.52 como modelo SVD. No caso de moléculas com simetria cilíndrica, devemos ter $\gamma=\lambda=0$, o que leva ao modelo de Maier-Saupe, dado pela eq.(2.28).

Em princípio, seria possível obter $\gamma$ e $\lambda$ desde que tenhamos informações sobre as moléculas, mas não há problema em supor que esses parâmetros possam ser escolhidos arbitrariamente, uma vez que a eq. (2.52) foi construída de forma bem geral. Uma escolha particularmente interessante é $\lambda=\gamma^{2}$, denominada aproximação de média geométrica, que resulta em

$$
V=-U_{0}(\mathbf{q}+\gamma \mathbf{b}) \cdot\left(\mathbf{q}^{\prime}+\gamma \mathbf{b}^{\prime}\right)
$$

Essa expressão pode ser interpretada como sendo um potencial de pares associado às forças de dispersão de London. Vale a pena lembrar que a parte anisotrópica do tensor de polarizabilidade molecular, dada pela eq.2.30), é uma combinação linear dos tensores de quadrupolo q e b. Para o tipo de forças de London que discutimos, a interação envolve apenas os tensores $\boldsymbol{\alpha}_{a}$ de cada molécula. Como resultado, o sistema possui um único parâmetro de ordem, e $\gamma$ estaria associado ao grau de biaxialidade molecular.

No caso mais geral, o modelo SVD leva a uma teoria molecular com dois parâmetros de ordem dados por $\mathbf{Q}=\langle\mathbf{q}\rangle$ e $\mathbf{B}=\langle\mathbf{b}\rangle$, uma vez que $\gamma$ e $\lambda$ podem assumir quaisquer valores. De fato, $\mathbf{Q}$ e $\mathbf{B}$ são tensores de quadrupolo que, na base dos seus respectivos autovetores, podem ser escritos nas formas diagonais

$$
\mathbf{Q}=\left[\begin{array}{ccc}
-\frac{1}{2}\left(S_{1}+\eta_{1}\right) & & \\
& -\frac{1}{2}\left(S_{1}-\eta_{1}\right) & \\
& & S_{1}
\end{array}\right]
$$

e

$$
\mathbf{B}=\left[\begin{array}{ccc}
-\frac{1}{2}\left(S_{2}+\eta_{2}\right) & & \\
& -\frac{1}{2}\left(S_{2}-\eta_{2}\right) & \\
& & S_{2}
\end{array}\right],
$$


onde $S_{i}$ e $\eta_{i}$, com $i=1,2$, são parâmetros escalares. Um ponto importante é que Straley [15] já havia mencionado que uma interação de pares mais geral levaria a uma descrição termodinâmica em termos de quatro parâmetros escalares associados ao ordenamento das fases nemáticas. Embora pareça complicado, é possível chegar a algumas interpretações sobre esses tensores. Por exemplo, para moléculas uniaxiais, esperamos que $\mathbf{b} \equiv 0$, o que implica que Q é o único parâmetro de ordem relevante no problema. Assim, levando em consideração apenas as simetrias do parâmetro de ordem, nada impede que haja fases biaxiais num ensemble de moléculas intrinsecamente uniaxiais. Por outro lado, é razoável pensar que $\mathbf{B}$ indica a contribuição da biaxialidade local para o ordernamento do sistema, quer ele seja uniaxial ou biaxial.

É perfeitamento possível desenvolver uma abordagem de campo médio para o modelo SVD, como mostrado por Nascimento et al. [47]. De fato, considere o hamiltoninano definido numa rede completamente conectada,

$$
\mathcal{H}=-\frac{U_{0}}{2 N} \sum_{i, j=1}^{N} \sum_{\mu, \nu}\left[q_{i}^{\mu \nu} q_{j}^{\mu \nu}+\gamma\left(q_{i}^{\mu \nu} b_{j}^{\mu \nu}+b_{i}^{\mu \nu} q_{j}^{\mu \nu}\right)+\lambda b_{i}^{\mu \nu} b_{j}^{\mu \nu}\right]
$$

onde $i, j$ rotulam as partículas do sistema e $\mu, \nu=x, y, z$ especificam os eixos do sistema de referência. Mais uma vez, seguiremos o esquema de discretização das orientações proposto por Zwanzig [41], o que leva a um modelo de seis estados, com os eixos principais de um mesógeno dados por

1. $\vec{n}_{1}=( \pm 1,0,0), \vec{n}_{2}=(0, \pm 1,0), \vec{n}_{3}=(0,0, \pm 1)$

2. $\vec{n}_{1}=( \pm 1,0,0), \vec{n}_{2}=(0,0, \pm 1), \vec{n}_{3}=(0, \pm 1,0)$;

3. $\vec{n}_{1}=(0, \pm 1,0), \vec{n}_{2}=( \pm 1,0,0), \vec{n}_{3}=(0,0, \pm 1)$

4. $\vec{n}_{1}=(0, \pm 1,0), \vec{n}_{2}=(0,0, \pm 1), \vec{n}_{3}=( \pm 1,0,0)$

5. $\vec{n}_{1}=(0,0, \pm 1), \vec{n}_{2}=( \pm 1,0,0), \vec{n}_{3}=(0, \pm 1,0)$

6. $\vec{n}_{1}=(0,0, \pm 1), \vec{n}_{2}=(0, \pm 1,0), \vec{n}_{3}=( \pm 1,0,0)$.

De posse desses estados, os tensores moleculares q e b são dados por matrizes diagonais, simplificando bastante os cálculos. Assim, a função canônica de partição pode ser escrita 
como

$$
Z=\operatorname{Tr} \exp \left\{\frac{1}{2 N T} \sum_{\mu}\left[\left(\sum_{i=1}^{N}\left(q_{i}^{\mu \mu}+\gamma b_{i}^{\mu \mu}\right)\right)^{2}+\omega^{2}\left(\sum_{i=1}^{N} b_{i}^{\mu \mu}\right)^{2}\right]\right\}
$$

onde $T=\left(\beta U_{0}\right)^{-1}$ é uma temperatura reduzida, o traço indica a soma sobre todos os estados orientacionais das moléculas, e também introduzimos um novo parâmetro $\omega^{2}=$ $\lambda-\gamma^{2}$. Do ponto de vista técnico, $\omega^{2}$ nos permite identificar quando devemos usar um tratamento de campo médio via identidades gaussianas $\left(\omega^{2}>0\right)$, ou se é mais apropriado utilizar as representações integrais da função delta de Dirac $\left(\omega^{2}<0\right)$. Em particular, $\omega^{2}=0$ corresponde ao caso de forças de dispersão de London.

Vamos nos restringir aos casos onde $\omega^{2}>0$, que englobam as situações comumente investigadas. Logo, de posse de um conjunto de identidades gaussianas do tipo

$$
\int_{-\infty}^{+\infty} \frac{d x}{\sqrt{\pi}} \exp \left(-x^{2}+2 a x\right)=\exp \left(a^{2}\right)
$$

a função de partição na eq.(2.58) torna-se

$$
\begin{aligned}
Z= & \frac{1}{\pi^{3}}\left(\prod_{\mu} \int d x_{\mu} d y_{\mu}\right) \exp \left[-\sum_{\mu}\left(x_{\mu}^{2}+y_{\mu}^{2}\right)\right] \times \\
& \times \operatorname{Tr}\left\{\exp \left[\sqrt{\frac{2}{N T}} \sum_{\mu}\left(x_{\mu} \sum_{i}\left(q_{i}^{\mu \mu}+\gamma b_{i}^{\mu \mu}\right)+\omega y_{\mu} \sum_{i} b_{i}^{\mu \mu}\right)\right]\right\}
\end{aligned}
$$

Seguindo o procedimento padrão, vamos fazer uma mudança de variáveis dada por

$$
\frac{X_{\mu}}{T}=\sqrt{\frac{2}{N T}} x_{\mu} \quad \text { e } \quad \frac{Y_{\mu}}{T}=\sqrt{\frac{2}{N T}} y_{\mu}
$$

Então, calculando o traço sobre todas as orientações moleculares, a função de partição assume a forma

$$
Z=\prod_{\mu}\left(\frac{N}{2 \pi T} \int d X_{\mu} d Y_{\mu}\right) \exp \left(-\frac{N \phi}{T}\right)
$$

onde

$$
\phi=\frac{1}{2} \sum_{\mu}\left(X_{\mu}^{2}+Y_{\mu}^{2}\right)+\frac{1}{3} \sum_{\mu} X_{\mu}-T \ln 2-T \ln \sigma_{0}
$$

é o funcional da energia livre para a versão de campo médio do modelo SVD discretizado, 
com

$$
\begin{aligned}
\sigma_{0}= & e^{\frac{X_{1}}{T}} \cosh \left[\frac{\gamma\left(X_{2}-X_{3}\right)+\omega\left(Y_{2}-Y_{3}\right)}{T}\right]+ \\
& +e^{\frac{X_{2}}{T}} \cosh \left[\frac{\gamma\left(X_{3}-X_{1}\right)+\omega\left(Y_{3}-Y_{1}\right)}{T}\right]+ \\
& +e^{\frac{X_{3}}{T}} \cosh \left[\frac{\gamma\left(X_{1}-X_{2}\right)+\omega\left(Y_{1}-Y_{2}\right)}{T}\right]
\end{aligned}
$$

Uma vez com o funcional em mãos, precisamos determinar os pontos estacionários de $\phi$ com respeito a $X_{\mu}$ e $Y_{\mu}$, dados pelas condições

$$
\frac{\partial \phi}{\partial X_{\mu}}=0 \quad \text { e } \quad \frac{\partial \phi}{\partial Y_{\mu}}=0
$$

Como resultado, chegamos a um sistema de equações autoconsistentes que satisfazem a condição de traço nulo,

$$
\sum_{\mu} X_{\mu}=\sum_{\mu} Y_{\mu}=0
$$

Na prática, obtemos um sistema de quatro equações com quatro incógnitas, que pode ser tratado numericamente sem grandes problemas.

Um olhar cuidadoso sobre a eq. 2.59 permite identificar que é possível fazer mais uma mudança de variáveis da forma $X_{\mu}=Q_{\mu}+\gamma B_{\mu}$ e $Y_{\mu}=\omega B_{\mu}$, que têm um significado físico sugestivo. De posse dessas novas variáveis, e refazendo todo o formalismo padrão de cálculo da função de partição, chegamos a um funcional da energia livre dado por

$$
\phi=\frac{1}{2} \sum_{\mu}\left(Q_{\mu}^{2}+2 \gamma Q_{\mu} B_{\mu}+\lambda B_{\mu}^{2}\right)+\frac{1}{3} \sum_{\mu}\left(Q_{\mu}+\gamma B_{\mu}\right)-T \ln 2-T \ln \bar{\sigma}_{0}
$$

com

$$
\begin{aligned}
\bar{\sigma}_{0}= & \exp \left(\frac{Q_{1}+\gamma B_{1}}{T}\right) \cosh \left[\frac{\gamma\left(Q_{2}-Q_{3}\right)+\lambda\left(B_{2}-B_{3}\right)}{T}\right]+ \\
& +\exp \left(\frac{Q_{2}+\gamma B_{2}}{T}\right) \cosh \left[\frac{\gamma\left(Q_{3}-Q_{1}\right)+\lambda\left(B_{3}-B_{1}\right)}{T}\right]+ \\
& +\exp \left(\frac{Q_{3}+\gamma B_{3}}{T}\right) \cosh \left[\frac{\gamma\left(Q_{1}-Q_{2}\right)+\lambda\left(B_{1}-B_{2}\right)}{T}\right]
\end{aligned}
$$

Novamente, temos que obter os valores de $Q_{\mu}$ e $B_{\mu}$ que minimizam o funcional $\phi$. Através de um cálculo direto, é simples mostrar que os pontos estacionários de $\phi$ são tais que

$$
\sum_{\mu} Q_{\mu}=\sum_{\mu} B_{\mu}=0
$$




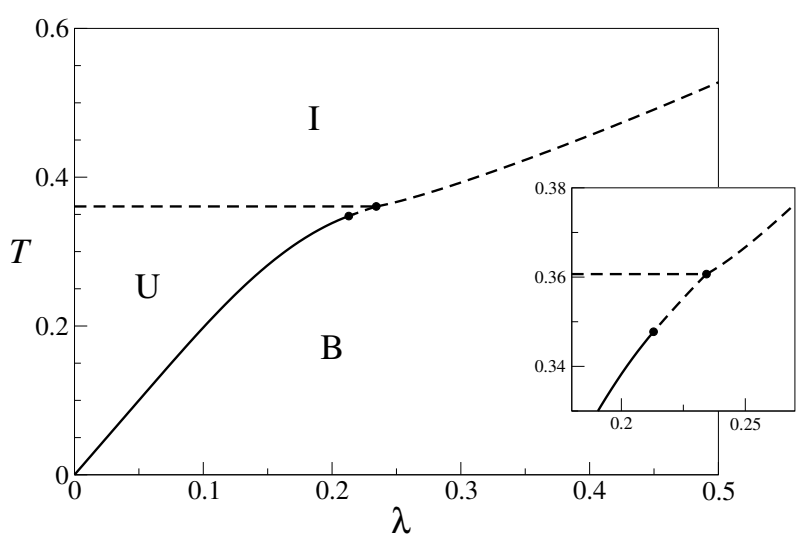

Figura 2.7: Diagrama de fases do modelo SVD discretizado, em termos da temperatura reduzida $T$ e do parâmetro $\lambda$. I: isotrópica. U: uniaxial. B: biaxial. O modelo apresenta pontos tricrítico e triplo, mas não tem ponto de Landau.

o que mostra, novamente, a condição de traço nulo dos parâmetros de ordem.

Obviamente, conseguimos recuperar o modelo de Maier-Saupe para moléculas com simetria cilíndrica, quando $\gamma=\lambda=0$, escrito de forma apropriada para fases uniaxiais calamíticas. Também obtemos, para $\lambda=\gamma^{2}$, o modelo de seis estados para objetos biaixais no contexto de forças de dispersão. Outro ponto importante, mas que não exploramos, é a possibilidade de escrever uma expansão do tipo Landau-de Gennes para o modelo SVD, mais ou menos na linha do trabalho de De Matteis et al. [48] para o caso de graus de liberdade contínuos.

Um caso particular muito curioso do modelo SVD ocorre quando $\gamma=0$ e $\lambda>0$, que foi uma situação investigada por Sonnet, Virga e Durand [16]. Para esta escolha de parâmetros, podemos aplicar sem problemas o formalismo das identidades gaussianas, o que significa que podemos usar o funcional dado pela eq. 2.66) dentro da região $(\gamma, \lambda)$ de interesse. Como resultado, obtemos o diagrama de fases mostrado na figura 2.7, onde aparecem as fases isotrópica (I), nemática uniaxial (U) e nemática biaxial (B). As linhas tracejadas indicam transições de primeira ordem e as linhas contínuas representam transições de segunda ordem.

Qualitativamente, nossa versão discretizada é consistente com os resultados obtidos por Sonnet, Virga e Durand [16]. A fronteira uniaxial-biaxial possui um ponto tricrítico, onde a transição muda de ordem. Além disso, também aparece um ponto triplo onde as fases isotrópica, uniaxial e biaxial coexistem. Note que existe uma transição direta, de 


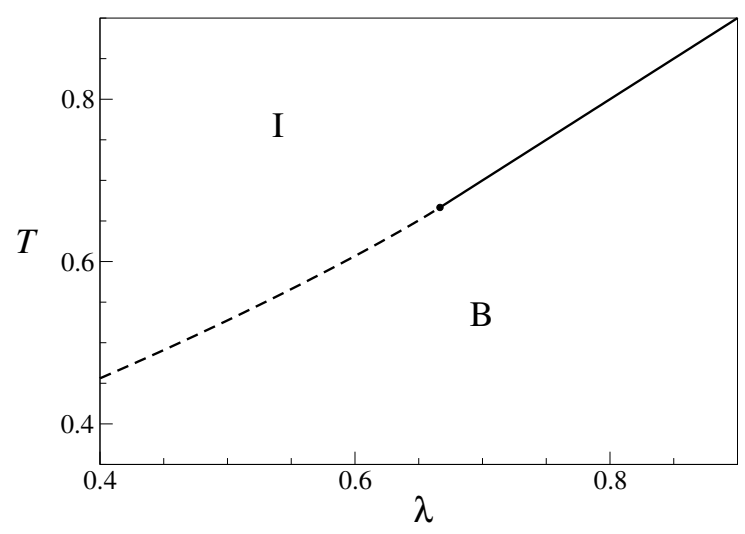

Figura 2.8: Diagrama de fases do modelo SVD discretizado, mostrando a região onde aparece um segundo ponto tricrítico.

primeira ordem, da fase biaxial para a fase isotrópica. Ou seja, para $\gamma=0$ e $\lambda>0$, o modelo SVD não apresenta um ponto multicrítico de Landau. De fato, existe um segundo ponto tricrítico, como mostrado na figura 2.8. Todos esses resultados são bem diferentes daqueles obtidos para o modelo de seis estados dado pela eq.2.32, que corresponde à aproximação de média geométrica $\left(\lambda=\gamma^{2}\right)$, cujo diagrama de fases mostramos na figura 2.5 .

Neste capítulo, estudamos as formulações efetivas mais simples usadas no tratamento de campo médio das fases nemáticas uniaxiais e biaxiais. De uma forma geral, é possível escrever um potencial de pares, dado por uma forma quadrática em termo de tensores de quadrupolo moleculares. Tal modelo é capaz de descrever casos particulares de interesse físico, como o que envolve forças de dispersão de London. No próximo capítulo, generalizaremos este caso para investigar uma mistura binária de mesógenos uniaxiais e biaxiais. 


\section{Modelo para mistura binária de}

\section{objetos uniaxiais e biaxiais}

Neste capítulo apresentaremos uma generalização da versão de campo médio do modelo de Maier-Saupe para uma mistura binária constituída de elementos intrinsecamente uniaxiais e intrinsecamente biaxiais. Então, adotando a discretização das orientações, calcularemos o funcional da energia livre e estabeleceremos contato com as teorias de Landau-de Gennes. Finalmente, apresentaremos os diagramas de fases para os diversos valores de parâmetros do sistema.

\subsection{Misturas binárias e fases nemáticas}

No capítulo anterior vimos que é possível obter mesofases nemáticas biaxiais a partir de modelos hamiltonianos onde os objetos elementares são intrinsecamente biaxiais 1 interagindo através de forças atrativas. Para esses tipos de sistemas, o diagrama de fases, numa formulação canônica, é constituído pela temperatura e por um parâmetro associado ao grau de assimetria do mesógeno. Então, mostramos que, dependendo do modelo em questão, temos fases uniaxiais e biaxiais, além de pontos multicríticos, como o ponto de Landau e pontos tricríticos.

Contudo, exite uma abordagem alternativa para estudar o comportamento termodinâmico de fases nemáticas biaxiais. Essa abordagem consiste em analisar misturas binárias formadas por objetos com formas distintas. Possivelmente, o caso mais conhe-

\footnotetext{
${ }^{1}$ Mais especificamente, objetos com simetria $D_{2 h}$.
} 
cido e discutido na literatura são as chamadas misturas de cilindros e discos, que são uma das diversas maneiras de definir uma mistura de objetos uniaxiais prolatos e oblatos 2 , Em sistemas desse tipo, uma fase biaxial seria possível quando, em média, mesógenos distintos tivessem eixos de simetria orientados perpendicularmente.

Existe muito interesse no comportamento termodinâmico dessas misturas, tanto do ponto de vista teórico [18, 19; 20; 21; 49] como experimental [8; 9; 10; 50, 51]. Em particular, Yu e Saupe [8] é, possivelmente, o primeira trabalho experimental que mostra a existência de uma fase biaxial numa mistura ternária liotrópica, cujo diagrama de fases é dado em termos da temperatura e da concentração de uma das componentes da mistura. Nesse diagrama, a fase biaxial aparece entre duas fases uniaxiais, uma calamítica e outra discótica. Mais recentemente, um arranjo de fases semelhante foi obtido por Akpinar et al. [10] numa mistura liotrópica quartenária. Note que esse tipo de sequência de fases é consistente com os resultados obtidos para modelo de objetos biaxiais interagindo através de forças de dispersão discutido na secão 2.2. Porém, tal sequência de fases também pode ser obtida a partir de uma mistura binária de objetos uniaxiais prolatos e oblatos [21; 51].

De fato, é perfeitamente possível descrever essas misturas binárias através de modelos de rede, do tipo Maier-Saupe, com graus de liberdade adicionais que representam as formas ${ }^{3}$ dos mesógenos [18; 19; 20; 21]. O problema é que temos que lidar com dois tipos de variáveis microscópicas: um associado à orientação e outro que descreveria o tipo de objeto. Do ponto de vista da mecânica estatística, como discutido no trabalho de do Carmo et al. [21], é possível tratar os graus de liberdade associados à forma de duas maneiras distintas: como variáveis aleatórias do tipo annealed ou do tipo quenched.

No contexto de sistemas desordenados, variáveis do tipo annealed são mais apropriadas para descrever estados microscópicos cujos tempos de relaxação são curtos, que é uma situação típica de sistemas fluidos. Por outro lado, sistemas sólidos são melhor representados por variáveis quenched, mais apropriadas para tempos de relaxação muito longos. É importante ter em mente que a maneira como lidamos com os diferentes graus liberdades pode afetar o digrama de fases. De fato, no caso de um modelo de Maier-Saupe para mistura de cilindros e discos, do Carmo et al. [21] mostraram que a estabilidade da fase

\footnotetext{
${ }^{2}$ Outro exemplo seriam esferóides com excentricidades distintas.

${ }^{3}$ Rigorosamente, a simetria dos objetos.
} 
biaxial estaria associada ao tipo de variável que descreve a forma dos objetos: estável para variáveis fixas, do tipo quenched, e instável para variáveis móveis, do tipo annealed. A discussão dessas diferenças foi uma contribuição importante para entender os resultados aparentemente conflitantes entre os trabalhos de Henriques e Henriques [20, que tratam a mistura numa formulação quenched, e de Palffy-Muhoray e colaboradores [18; 19], onde a desordem é representada por variáveis do tipo annealed.

Aqui, iremos dar um passo adiante no entendimento dos efeitos dos graus de liberdade desordenados, associados à forma, sobre as fases nemáticas em misturas binárias de objetos com simetrias diferentes. Seguiremos uma abordagem intermediária entre sistemas constituídos por mesógenos biaxiais e misturas de duas componentes formadas por objetos uniaxiais. Mais especificamente, vamos estudar uma mistura binária de objetos intrinsecamente biaxiais e objetos intrinsecamente uniaxiais. Além disso, vamos restringir nossa análise a uma formulação annealed do problema, haja vista que estamos interessados em sistemas fluidos.

\subsection{Mistura binária de objetos uniaxiais e biaxiais}

Considere uma mistura binária de elementos intrinsecamente uniaxiais e elementos intrinsecamente biaxiais. Uma versão de campo médio para a mistura pode ser formulada com o auxílio do modelo de Maier-Saupe para objetos biaxiais eq. 2.32). Assim, vamos introduzir um hamiltoniano da forma

$$
\mathcal{H}=-\frac{\epsilon}{2 N} \sum_{i, j=1}^{N} \sum_{\mu, \nu=1}^{3} \Omega_{i}^{\mu \nu}\left(\Delta_{i}\right) \Omega_{j}^{\mu \nu}\left(\Delta_{j}\right),
$$

onde $\epsilon$ é uma constante de acoplamento entre os momentos de quadrupolo $\Omega_{i}\left(\Delta_{i}\right)$ e $\Omega_{j}\left(\Delta_{j}\right)$, definidos sobre os sítios $i$ e $j$ de uma rede. Aqui também seguiremos a discretização das orientações proposta por Zwanzig [41], o que simplifica bastante os cálculos. Não é difícil perceber que os momentos de quadrupolo $\Omega_{i}\left(\Delta_{i}\right)$ são dados por matrizes diagonais. Uma representação para essas matrizes seria do tipo

$$
\left(\begin{array}{ccc}
-1+\Delta_{i} & & \\
& -1-\Delta_{i} & \\
& & 2
\end{array}\right)
$$




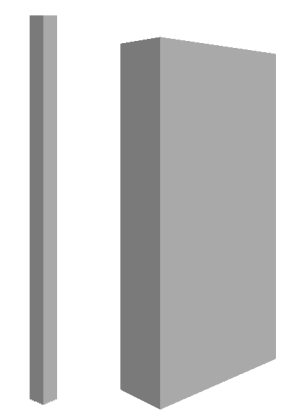

Figura 3.1: Objetos com formas distintas e eixos maiores orientados paralelamente.

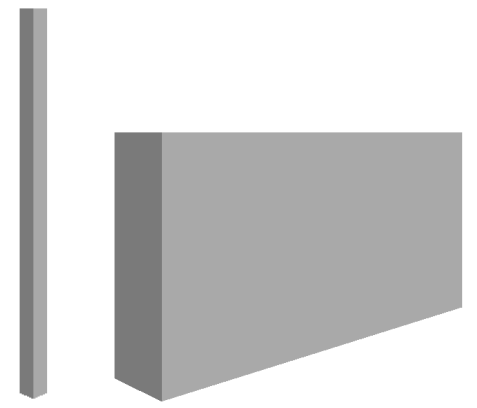

Figura 3.2: Objetos com formas diferentes e eixos maiores orientados perpendicularmente.

É claro que os demais estados possíveis para $\Omega_{i}\left(\Delta_{i}\right)$ seriam formas diagonais obtidas a partir das permutações dos elementos da diagonal principal de (3.2).

Como estamos interessados numa mistura binária, é conveniente escrever o parâmetro de biaxialidade como $\Delta_{i}=n_{i} \Delta$, onde

$$
n_{i}= \begin{cases}1, & \text { se o objeto é biaxial } \\ 0, & \text { se o objeto é uniaxial. }\end{cases}
$$

A introdução da variável $n_{i}$ permite levarmos em conta o grau de liberdade associado à forma do objeto (figuras 3.1 e 3.2.

Numa formulação recozida (annealed) do problema, vamos supor que existam $N_{1}$ objetos intrinsecamente biaxiais e $N_{2}$ objetos intrinsecamente uniaxiais, de tal forma que $N=N_{1}+N_{2}$ é o número de partículas do sistema. Logo, a função de partição canônica da mistura é dada por

$$
Z_{a}=\sum_{\left\{\boldsymbol{\Omega}_{i}\right\}} \sum_{\left\{n_{i}\right\}}^{\prime} \exp \left[\frac{\beta \epsilon}{2 N} \sum_{i, j=1}^{N} \sum_{\mu=1}^{3} \Omega_{i}^{\mu \mu}\left(n_{i}\right) \Omega_{j}^{\mu \mu}\left(n_{j}\right)\right],
$$

onde $\beta \epsilon=1 / T$, sendo que $T$ é a temperatura reduzida, e a segunda soma obedece à 
restrição

$$
\sum_{i=1}^{N} n_{i}=N_{1}
$$

Assim como ocorre no caso da mistura de cilindros e discos [21], podemos lidar com esse vínculo migrando para o ensemble grande canônico através da introdução de um potencial químico. Após algumas manipulações algébricas, a grande função de partição da mistura pode ser escrita como

$$
\Xi=\sum_{\left\{\boldsymbol{\Omega}_{i}\right\}} \sum_{\left\{n_{i}\right\}} \exp \left\{\frac{\beta \epsilon}{2 N} \sum_{\mu=1}^{3}\left[\sum_{i=1}^{N} \Omega_{i}^{\mu \mu}\left(n_{i}\right)\right]^{2}+\beta \epsilon \mu \sum_{i=1}^{N} n_{i}\right\},
$$

onde $\mu$ é o potencial químico reduzido que controla a quantidade de elementos biaxiais no sistema.

Novamente, vamos fatorar a soma na exponencial em (3.5) com o auxílio de identidades gaussianas do tipo

$$
\exp \left(a_{\mu}^{2}\right)=\frac{1}{\sqrt{\pi}} \int_{-\infty}^{+\infty} d x_{\mu} \exp \left(-x_{\mu}^{2}+2 a_{\mu} x_{\mu}\right)
$$

resultando na equação

$$
\begin{aligned}
\Xi & =\frac{1}{\pi^{3 / 2}}\left(\prod_{\mu=1}^{3} \int_{-\infty}^{+\infty} d x_{\mu}\right) \sum_{\left\{\boldsymbol{\Omega}_{i}\right\}} \sum_{\left\{n_{i}\right\}} \exp \left[-\sum_{\mu=1}^{3} x_{\mu}^{2}\right. \\
& \left.+\sqrt{\frac{2 \beta \epsilon}{N}} \sum_{\mu=1}^{3} x_{\mu} \sum_{i} \Omega_{i}^{\mu \mu}\left(n_{i}\right)+\beta \epsilon \mu \sum_{i} n_{i}\right]
\end{aligned}
$$

onde vamos fazer uma mudança de variáveis do tipo

$$
x_{\mu}^{2}=\frac{N \beta \epsilon}{2} q_{\mu}^{2} .
$$

Por fim, a grande função de partição assume a representação integral

$$
\Xi=\int d \mathbf{q} \exp (-N \beta \epsilon \psi)
$$

onde

$$
\begin{aligned}
\psi= & \frac{1}{2}\left(q_{1}^{2}+q_{2}^{2}+q_{3}^{2}\right)+\left(q_{1}+q_{2}+q_{3}\right)-T \ln 2+ \\
& -T \ln \left\{\exp \left(\frac{3 q_{1}}{T}\right)\left[1+z \cosh \left(\frac{\left(q_{2}-q_{3}\right) \Delta}{T}\right)\right]\right. \\
& +\exp \left(\frac{3 q_{2}}{T}\right)\left[1+z \cosh \left(\frac{\left(q_{3}-q_{1}\right) \Delta}{T}\right)\right] \\
& \left.+\exp \left(\frac{3 q_{3}}{T}\right)\left[1+z \cosh \left(\frac{\left(q_{1}-q_{2}\right) \Delta}{T}\right)\right]\right\}
\end{aligned}
$$


é o chamado funcional do grande potencial termodinâmico e $z=\exp (\mu / T)$ é a fugacidade. Para fazermos contato com a termodinâmica devemos tomar o limite $N \rightarrow \infty$ e aplicar o método da integração assintótica de Laplace.

Os pontos estacionários de $\psi$ são obtidos a partir das condições de extremo $\partial \psi / \partial q_{i}=0$. Essas condições levam a um sistema de equações não-lineares da forma $q_{i}=f_{i}\left(q_{1}, q_{2}, q_{3}\right)$. Não é difícil mostrar que esse sistema obedece à propriedade de traço nulo, $q_{1}+q_{2}+q_{3}=0$. Mais uma vez, vamos fazer a mudança de variáveis proposta em 2.40). Portanto, em termos de $S$ e $\eta$, o funcional $\psi$ é dado por

$$
\begin{aligned}
\psi= & \frac{1}{4}\left(3 S^{2}+\eta^{2}\right)-T \ln 2+ \\
& -T \ln \left\{\exp \left[\frac{-3(S+\eta)}{2 T}\right]\left[1+z \cosh \left(\frac{(-3 S+\eta) \Delta}{2 T}\right)\right]+\right. \\
& +\exp \left[\frac{-3(S-\eta)}{2 T}\right]\left[1+z \cosh \left(\frac{(3 S+\eta) \Delta}{2 T}\right)\right]+ \\
& \left.+\exp \left(\frac{3 S}{T}\right)\left[1+z \cosh \left(\frac{\eta \Delta}{T}\right)\right]\right\} .
\end{aligned}
$$

Novamente, a partir do cálculo dos extremos de $\psi=\psi(S, \eta)$, obtemos um sistema de equações acopladas do tipo $S=f_{1}(S, \eta)$ e $\eta=f_{2}(S, \eta)$. Em geral, esse sistema admite mais de uma solução. Contudo, estamos interessados na solução para a qual $\psi$ é mínino, dados a temperatura $T$, a biaxialidade $\Delta$ e o potencial químico $\mu$.

\subsection{Conexão com a teoria de Landau-de Gennes}

Antes de apresentarmos os diagramas de fases da mistura binária, vamos fazer contato com a teoria fenomenológica de Landau-de Gennes [1; 4; 46], seguindo a mesma abordagem descrita em 2.2.1. Vamos começar identificando o parâmetro de ordem do sistema, que é um tensor simétrico de traço nulo,

$$
\mathbf{Q}=\left(\begin{array}{ccc}
q_{1} & & \\
& q_{2} & \\
& & q_{3}
\end{array}\right) \quad, \quad \operatorname{Tr} \mathbf{Q}=0
$$

Em seguida, introduzimos os invariantes do tensor $\mathbf{Q}$, dados por $I_{n}=\operatorname{Tr} \mathbf{Q}^{n}$, com $n=$ $1,2,3, \cdots$. Porém, devido às propriedades de simetria de $\mathbf{Q}$, todos os invariantes $I_{n}$ 
dependem apenas de $I_{2}=\operatorname{Tr} \mathbf{Q}^{2}$ e $I_{3}=\operatorname{Tr} \mathbf{Q}^{3}$. Como o funcional $\psi$ é um invariante rotacional, podemos escrever uma expansão do tipo

$$
\psi=\psi_{0}+\frac{A}{2} I_{2}+\frac{B}{3} I_{3}+\frac{C}{4} I_{2}^{2}+\frac{D}{5} I_{2} I_{3}+\frac{E}{6} I_{2}^{3}+\frac{E^{\prime}}{6} I_{3}^{2}+\cdots,
$$

sendo que $\psi_{0}$ é a energia livre associada à fase isotrópica. Os coeficientes da expansão (3.13) são dados por

$$
\begin{gathered}
A=1-\frac{3+z\left(3+\Delta^{2}\right)}{T(1+z)}, \\
B=\frac{9}{2} \frac{z\left(\Delta^{2}-1\right)-1}{T^{2}(1+z)}, \\
C=\frac{9+z\left[18+9 z+6(1+z) \Delta^{2}-(1-z) \Delta^{4}\right]}{4 T^{3}(1+z)^{2}}, \\
D=\frac{15}{16} \frac{9+z\left[18-6 \Delta^{2}+\Delta^{4}-3 z\left(-3+2 \Delta^{2}+\Delta^{4}\right)\right]}{T^{4}(1+z)^{2}} \\
E=\frac{1053-z\left(c_{0}+z c_{1}+z^{2} c_{2}\right)}{480 T^{5}(1+z)^{3}}
\end{gathered}
$$

onde

$$
\begin{aligned}
& c_{0}=-3159-1215 \Delta^{2}+225 \Delta^{4}-11 \Delta^{6}, \\
& c_{1}=-3159-2430 \Delta^{2}-90 \Delta^{4}+68 \Delta^{6},
\end{aligned}
$$

e

$$
\begin{gathered}
c_{2}=-1053-1215 \Delta^{2}-315 \Delta^{4}-41 \Delta^{6}, \\
E^{\prime}=\frac{243+z\left(d_{0}+z d_{1}\right)}{240 T^{5}(1+z)^{2}}
\end{gathered}
$$

sendo que

$$
d_{0}=486-405 \Delta^{2}-45 \Delta^{4}+\Delta^{6}
$$

e

$$
d_{1}=243-405 \Delta^{2}+225 \Delta^{4}+\Delta^{6}
$$

As coordenadas do ponto de Landau são obtidas através das condições usuais $A=0$ e $B=0$, que levam ao resultado

$$
T_{L}=4, \quad \mu_{L}=-T_{L} \ln \left(\Delta^{2}-1\right)
$$

Ou seja, não existe ponto de Landau para $\Delta^{2}<1$. Além disso, a análise dos coeficientes da expansão sugere que o diagrama de fases da mistura apresenta um comportamento muito rico, como veremos na próxima seção. 


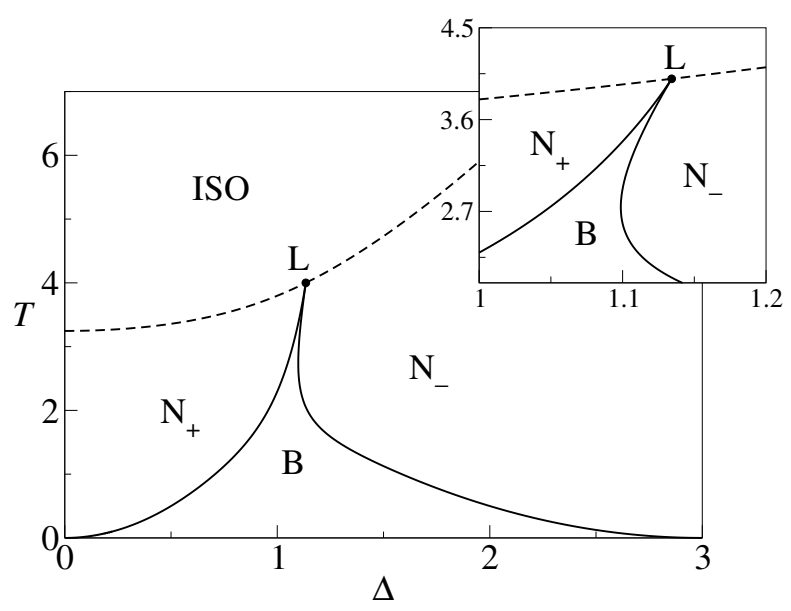

Figura 3.3: Diagrama de fases para $\mu=5$.

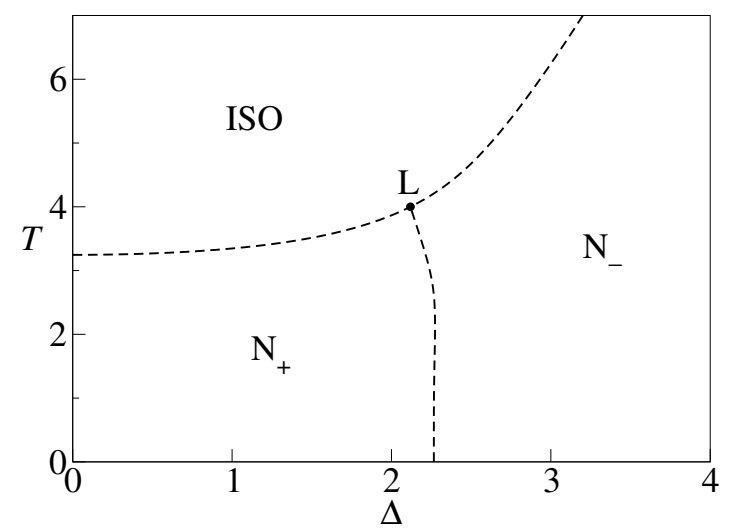

Figura 3.4: Diagrama de fases para $\mu=-5$.

\subsection{Diagramas de fases}

A figura 3.3 mostra o diagrama de fases no plano gerado pela temperatura $T$ e pela biaxialidade $\Delta$ para potencial químico $\mu=5$. As fases nemáticas uniaxiais $\mathrm{N}_{+}$e $\mathrm{N}_{-}$ estão separadas da fase isotrópica ISO por linhas de transição de primeira ordem (linhas tracejadas). Além disso, $\mathrm{N}_{+}$e $\mathrm{N}_{-}$estão separadas da fase biaxial B por linhas de transição de segunda ordem (linhas contínuas). Vemos que existe um comportamento de reentrância da fase B nas vizinhanças do ponto de Landau L. Este comportamento não aparece no caso uniforme, como pode ser visto na figura 2.5 .

A figura 3.4 exibe o diagrama de fases no plano temperatura $T$ versus grau de biaxialidade $\Delta$ para potencial químico $\mu=-5.0$. As fases nemáticas uniaxiais $\left(\mathrm{N}_{+}\right.$e $\left.\mathrm{N}_{-}\right)$ estão separadas da fase ISO por linhas de transição de primeira ordem (linhas tracejadas). 


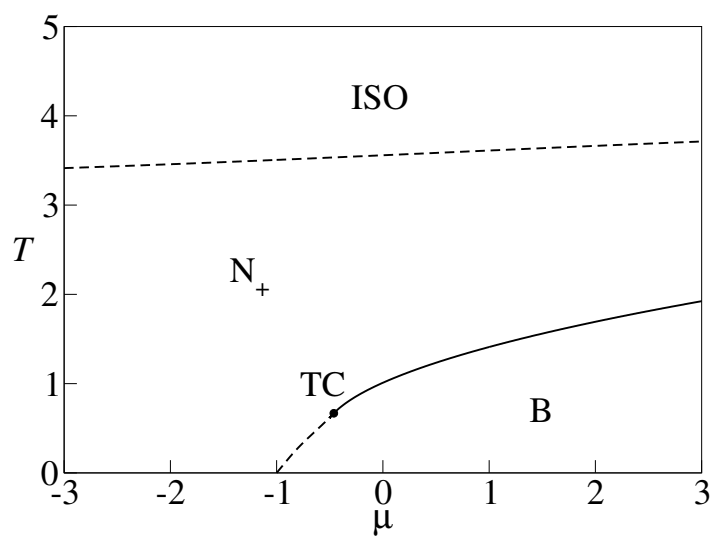

Figura 3.5: Diagrama de fases da mistura binária para $\Delta=1$.

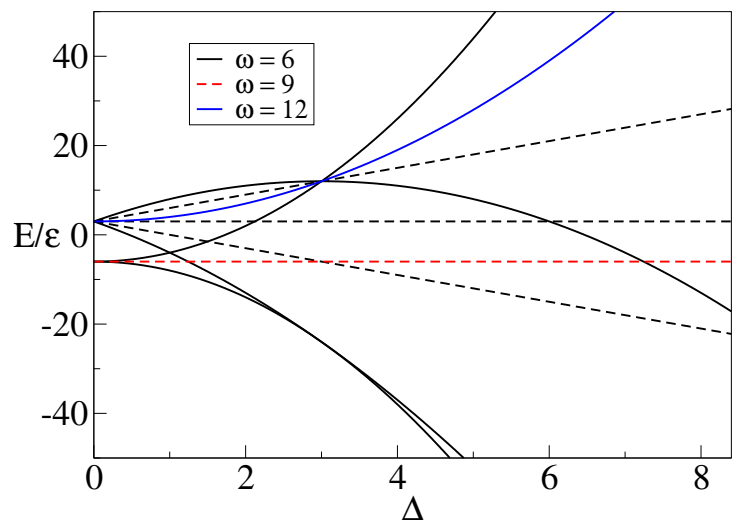

Figura 3.6: Níveis de energia da mistura binária como função do parâmetro de biaxialidade $\Delta$. As degenerescências dos níveis são indicadas por $\omega$.

Também existe uma fronteira de primeira ordem entre as fases $\mathrm{N}_{+}$e $\mathrm{N}_{-}$. Essas linhas de primeira ordem se encontram no ponto de Landau L. Não há fase biaxial estável para esse valor de potencial químico.

A figura 3.5 mostra o diagrama de fase no plano $T \times \mu$ para grau de biaxialidade $\Delta=1$. A fase $\mathrm{N}_{+}$apresenta uma fronteira de primeira ordem com a fase ISO. Não existe transição entre as fases B e ISO para potencial químico finito. Na região de baixas temperaturas, a fase $\mathrm{N}_{+}$exibe uma transição de primeira ordem para a fase B à medida que o potencial químico aumenta. Para temperaturas intermediárias, as fases $\mathrm{N}_{+}$e B estão separadas por transições de segunda ordem. Existe um ponto tricrítico TC ao longo da fronteira entre $\mathrm{N}_{+}$e B. O sistema apresenta diagramas de fases com topologias semelhantes à da figura 3.5 para biaxialidade na região $0<\Delta<1$.

Assim como fizemos no caso do sistema formado por objetos intrinsecamente biaxiais, 


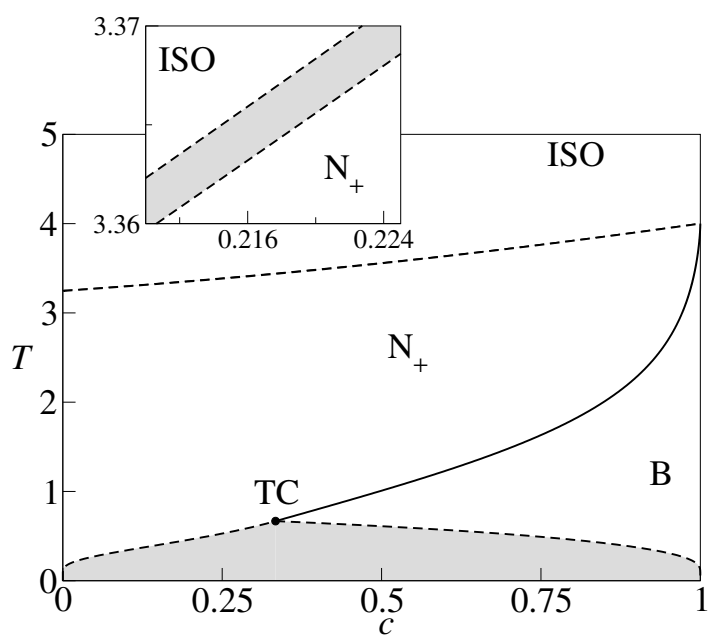

Figura 3.7: Diagrama de fases para $\Delta=1$.

é possível entender as diversas topologias dos diagramas de fases em termos dos níveis de energias da mistura (figura 3.6) como função do parâmetro de biaxialidade $\Delta$. As linhas contínuas indicam os níveis de energia de dois objetos biaxiais, enquanto que as linhas tracejadas representam os níveis de energia de dois objetos uniaxiais ou um objeto uniaxial com um objeto biaxial. Os valores das degenerescências dos níveis são dadas por $\omega$. Essas degenerescências podem influenciar a parte entrópica da energia livre do sistema, levando a mudanças na topologia dos diagramas de fases nas vizinhanças dos cruzamentos dos níveis.

Por outro lado, é interessante, do ponto de vista experimental, um diagrama de fases em termos da concentração em vez do potencial químico. Assim, a figura 3.6 apresenta o diagrama no plano temperatura $T$ contra a concentração $c$ de objetos biaxiais para $\Delta=1$. As linhas de transição de primeira ordem são descritas por regiões de coexistência em escala cinza. Contudo, as linhas críticas no plano $T \times \mu$ são mapeadas em linhas críticas no plano $T \times c$. Não existe transição direta entre as fases B e ISO para $\Delta<1$, o que é consistente com a figura 3.5. A transição ocorre apenas em $c=1$, que corresponde a um sistema biaxial puro. Na região de $\Delta<1$, as fases B e ISO estão sempre separadas pela fase $\mathrm{N}_{+}$para qualquer valor de concentração $c$, o que está de acordo com o cruzamento entre níveis, como mostrado na figura 3.6, e com o resultado dado por 3.25, obtido a partir da expansão de Landau-de Gennes.

A figura 3.8 exibe o diagrama de fases em termos temperatura reduzida $T$ e da con- 


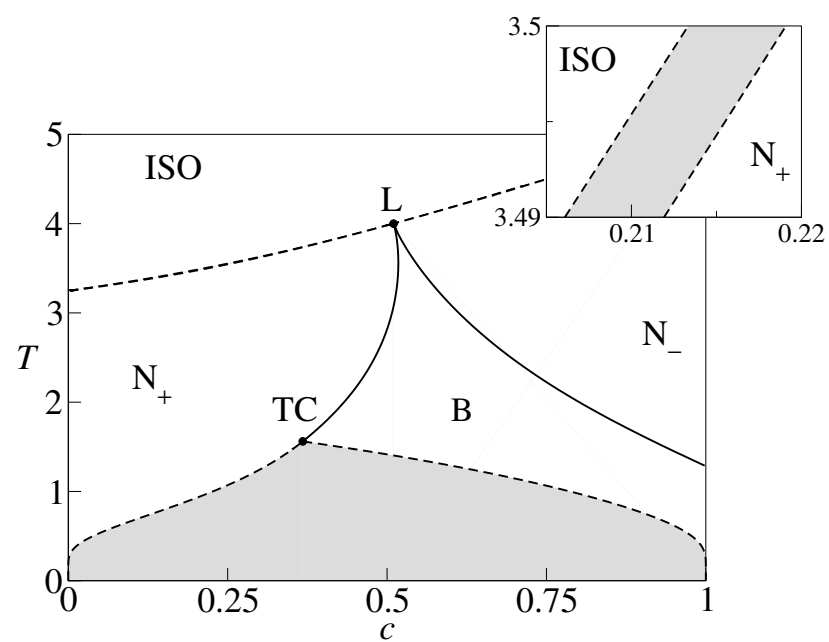

Figura 3.8: Diagrama de fases para $\Delta=1.4$.

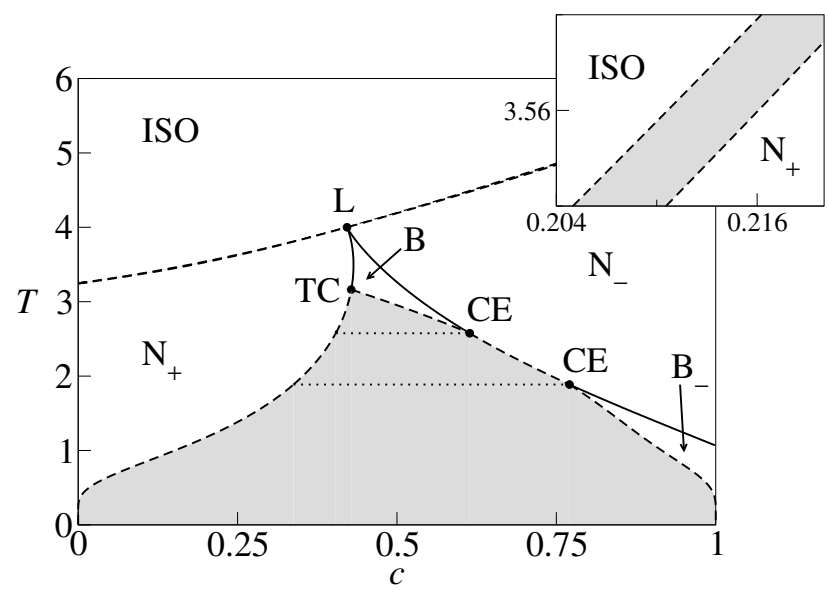

Figura 3.9: Diagrama de fases para $\Delta=1.54$.

centração $c$ para biaxialidade $\Delta=1.4$. Na região de baixas temperaturas, a fase $\mathrm{N}_{+}$sofre uma transição de primeira ordem para a fase B à medida que o concentração de objetos biaxiais aumenta, o que leva ao surgimento de uma região de coexistência $\mathrm{N}_{+}-\mathrm{B}$. Essa região termina num ponto tricrítico TC quando a temperatura aumenta. Por outro lado, a fronteira entre as fases $\mathrm{N}_{-}$e B é sempre de segunda ordem. A fase B é estável nas vizinhanças do ponto de Landau L, além de apresentar um comportamento de reentrância. Diagramas de fases semelhantes ao da figura 3.8 são encontrados para $1<\Delta \leq 1.5$.

De acordo com a figura 3.6, existe um cruzamento de níveis nas vizinhanças de $\Delta=$ 1.55, o que pode levar a mudanças na topologia do diagrama de fases. Então, com o objetivo de verificar se tal mudança ocorre, construímos o diagrama $T \times c$ para $\Delta=1.54$, como mostrado figura 3.9. De fato, esse diagrama de fase exibe uma topologia bastante 


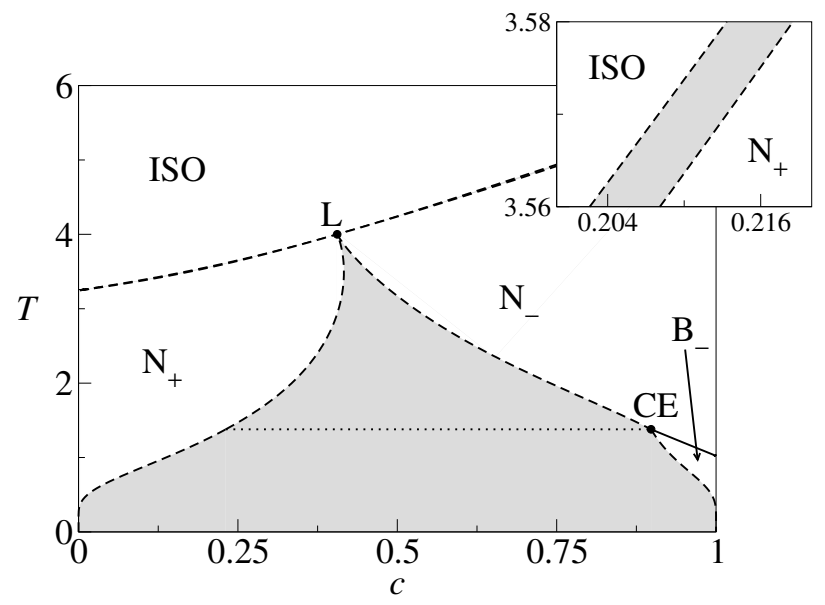

Figura 3.10: Diagrama de fases para $\Delta=1.57$.

complexa. Para baixas temperaturas e altas concentrações, aparece uma fase biaxial que denominamos de $\mathrm{B}_{-}$porque tem $S$ negativo, e que sofre uma transição de segunda ordem para a fase nemática uniaxial $N_{-}$quando a temperatura aumenta. Existe uma região de coexistência entre as fases $\mathrm{N}_{+}$e $\mathrm{B}_{-}$que termina num ponto crítico terminal $\mathrm{CE}$, a partir do qual surge outra região de coexistência, mas agora entre as fases $\mathrm{N}_{+}$e $\mathrm{N}_{-}$. Contudo, a região de coexistência $\mathrm{N}_{+}-\mathrm{N}_{-}$acaba em um segundo ponto crítico terminal associado a uma segunda fase biaxial B. Essa fase apresenta um região de coexistência com a fase $\mathrm{N}_{+}$que termina num ponto tricrítico TC, além de ter um comportamento reentrante nas vizinhanças do ponto de Landau L. Note que a figura 3.6 exibe duas fases nemáticas biaxiais estáveis em regiões desconexas do diagrama de fases.

Diagramas como o mostrado na figura 3.9 ocorrem apenas numa vizinhança pequena de $\Delta=1.55$. Quando aumentamos o grau de biaxialidade, encontramos diagramas semelhantes aos da figura 3.10, onde não existe fase biaxial estável nas vizinhanças do ponto de Landau. O que vemos é uma região de coexistência entre $\mathrm{N}_{+}$e $\mathrm{N}_{-}$nas proximidades de L. Contudo, a outra fase biaxial, $\mathrm{B}_{-}$, ainda está presente em baixas temperaturas e altas concentrações.

Uma outra maneira de entendermos as mudanças nos diagramas de fases é através das projeções dos diversos pontos multicríticos no plano gerado pela temperatura reduzida $T$ e pelo grau de biaxialidade $\Delta$, como mostrado na figura 3.11 . Para $\Delta \leq \Delta_{M_{1}}$, onde $\Delta_{M_{1}}$ é a abscissa do ponto $\mathrm{M}_{1}$, o sistema apresenta pontos tricríticos e críticos terminais, associados a fases nemáticas biaxiais, bem como pontos de Landau. Note que a linha de pontos 


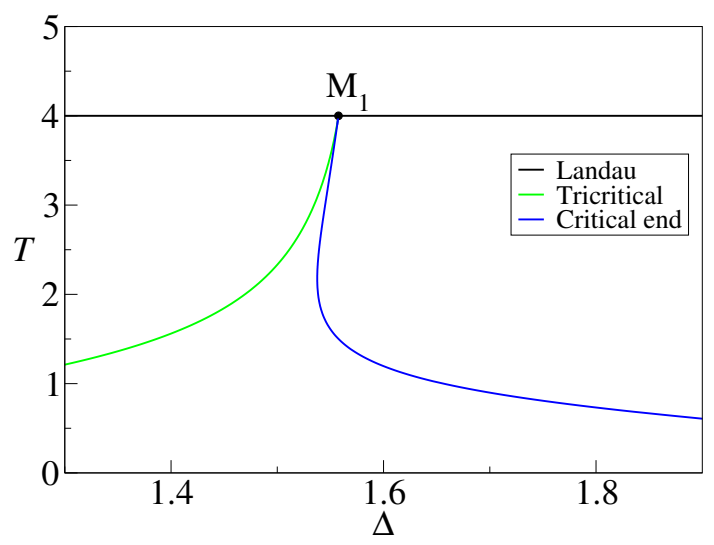

Figura 3.11: Pontos multicríticos no plano $T \times \Delta$.

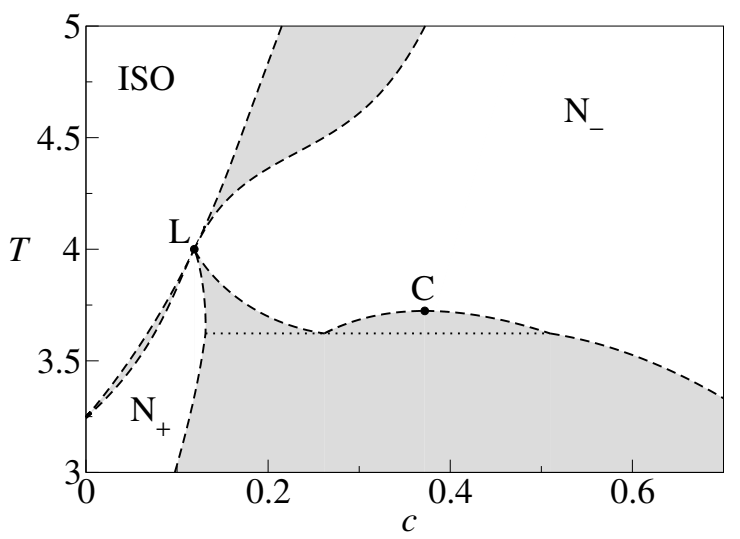

Figura 3.12: Diagrama de fases para $\Delta=2.9$.

críticos terminais tem um comportamento reentrante, o que está de acordo com as Figuras 3.9 3.10. Para $\Delta \geq \Delta_{M_{1}}$, encontramos pontos de Landau e pontos críticos terminais. Estes, também associados a uma fase biaxial (figura 3.10), apresentam temperaturas características que diminuem quando aumentamos a biaxialidade. As linhas de pontos de Landau, pontos tricríticos e pontos críticos terminais se encontram num ponto multicrítico de ordem superior $\mathrm{M}_{1}$. Como os pontos tricríticos e críticos terminais estão associados às fases biaxiais, estas não aparecem para $\Delta \geq \Delta_{M_{1}}$ nas proximidades do ponto de Landau. 


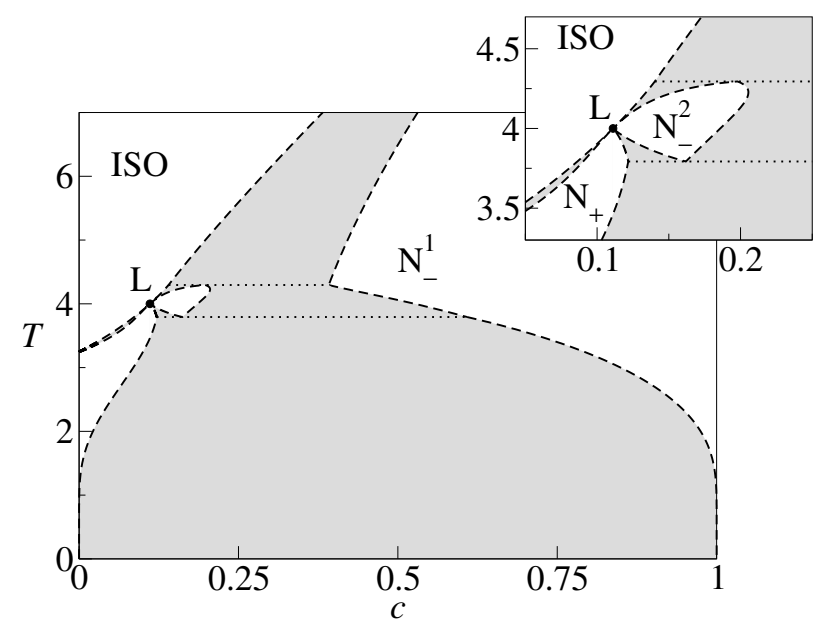

Figura 3.13: Diagrama de fases para $\Delta=3$.

Seguindo a ideia de que mudanças topológicas nos diagramas podem ocorrer nas vizinhanças dos cruzamentos dos níveis de energia (figura 3.6), estudamos o comportamento do sistema para diferentes valores de biaxialidade em torno de $\Delta=3$. Na figura 3.12 mostramos o diagrama para $\Delta=2.9$, que apresenta um ponto crítico simples, $\mathrm{C}$ e um ponto triplo, representado pela linha de conjugação. O ponto crítico C é o ponto terminal da região de coexistência entre duas fases uniaxiais discóticas. Já no ponto triplo ocorre a coexistência de um fase uniaxial calamítica com duas fases uniaxiais discóticas. Existe uma fase biaxial estável em altas concentrações e baixas temperaturas, mas tal fase ocupa uma região muito pequena do diagrama. Não encontramos fase biaxial estável nas vizinhanças do ponto de Landau L.

Na figura 3.13 temos o diagrama de fases para biaxialidade $\Delta=3$. De acordo com (3.2), podemos interpretar o sistema como sendo uma mistura de objetos uniaxiais prolatos e oblatos: basicamente um mistura binária de discos e cilindros. No entanto, é fácil perceber que as energias de interação disco-disco e cilindro-cilindro são diferentes, sendo que o acoplamento mais estável ocorre entre discos com a mesma orientação. Ou seja, temos uma mistura assimétrica de objetos uniaxiais, cujo diagrama de fases é bem diferente daquele associado a uma mistura simétrica [21]. No caso assimétrico (figura 3.13), encontramos duas fases uniaxiais discóticas, $\mathrm{N}_{-}^{1}$ e $\mathrm{N}_{-}^{2}$, dois pontos triplos (representados pelas linhas de conjugação), além do ponto de Landau L. O diagrama para o caso simétrico é mais simples [21], com apenas uma fase uniaxial discótica e um ponto de Landau. Assim como no caso simétrico, a mistura assimétrica não apresenta fase biaxial 


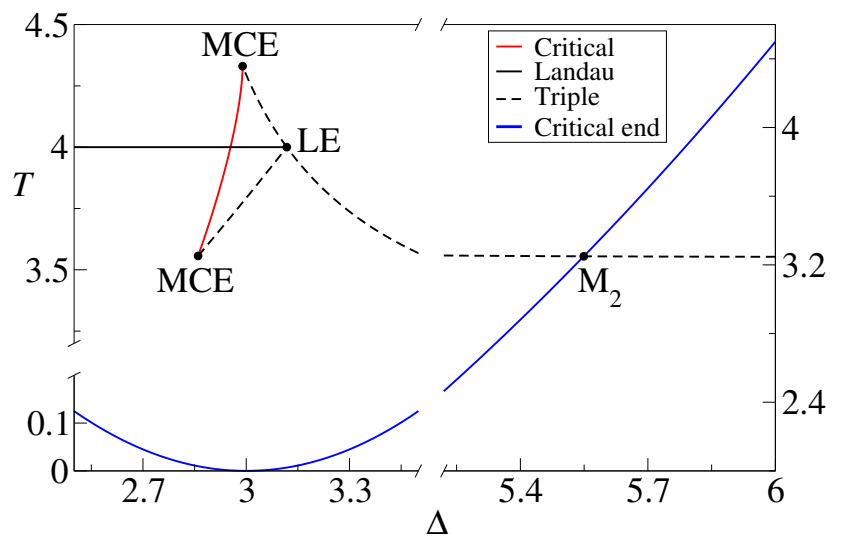

Figura 3.14: Pontos multicríticos no plano $T \times \Delta$.

estável para quaisquer domínios de temperatura e concentração.

Por fim, a figura 3.14 mostra as projeções dos diversos pontos multicríticos no plano temperatura reduzida $T$ contra o grau de biaxialidade $\Delta$. O ponto crítico $\mathrm{C}$ presente na figura 3.12 pertence a uma linha de pontos críticos simples que tem como fronteira dois pontos multicríticos terminais MCE. Cada um dos pontos MCE está associado a uma linha de pontos triplos (figura 3.13), que se encontram com a linha de Landau em um ponto que denominamos ponto terminal de Landau LE. Neste ponto há a coexistência da fase isotrópica, que é crítica, com uma fase uniaxial discótica não-crítica. Além disso, existe uma terceira linha de pontos triplos ligada ao ponto LE. Ou seja, a partir de um dado valor de $\Delta$, o ponto de Landau é substituído por um ponto triplo na região de altas temperaturas. Note que existe uma linha de pontos críticos terminais para baixas temperaturas, sendo que esses pontos estão associados a uma fase biaxial estável presente apenas em altas concentrações. Contudo, à medida que o grau de biaxialidade aumenta, a linha de pontos críticos terminais apresenta um comportamento ascendente no plano $T \times \Delta$, chegando a interceptar a linha de pontos triplos num ponto multicrítico de ordem superior, $\mathrm{M}_{2}$. O surgimento desse ponto indica que pode haver uma região de coexistência entre as fases isotrópica e biaxial.

De fato, a figura 3.15 apresenta o diagrama de fases para $\Delta=6$. Tivemos que modificar as escalas das concentrações para que as regiões de interesse fossem exibidas. Vemos que há uma região de coexistência entre as fases isotrópica e biaxial num dado domínio de temperatura. Como indicado na figura 3.14, existe um ponto triplo (linha de conjugação) onde as fases isotrópica, uniaxial e biaxial coexistem. Por outro lado, há um 


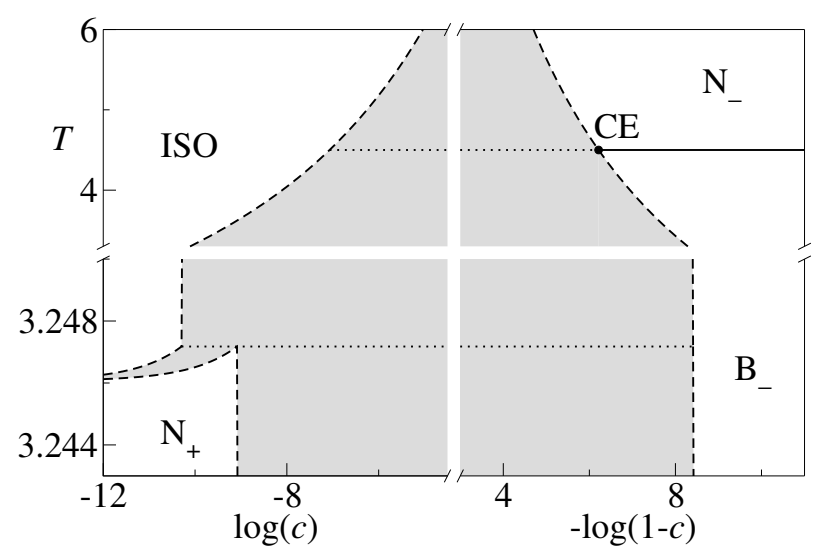

Figura 3.15: Diagrama de fases para $\Delta=6$.

ponto crítico terminal CE em altas temperaturas, associado à fase biaxial. Note que os pontos triplos e críticos terminais ocorrem em concentrações extremas. Acreditamos que esses resultados devem ser refinados com abordagens onde os efeitos de coordenação finita são considerados, como cálculos na árvore de Cayley e simulações de Monte Carlo. 


\section{Interações estéricas e nemáticos}

\section{uniaxiais}

Neste capítulo iremos estudar a transição nemático-isotrópica no contexto de sistemas de objetos anisotrópicos rígidos. Discutiremos a teoria desenvolvida por Onsager para partículas prolatas longas, também chamada de modelo de bastões rígidos, que é mais apropriada para sistemas em baixas densidades. Então, seguindo uma abordagem de campo médio, apresentaremos uma maneira de generalizar a teoria de Onsager, de tal forma que possamos descrever sistemas com densidades maiores e relação de aspecto finita.

\subsection{Sistemas de muitos corpos rígidos}

Há muito interesse nas propriedades termodinâmicas de sistemas formados por partículas não-esféricas que interagem através de potenciais do tipo caroço duro [31; 52]. Devido à ausência de interações atrativas, a temperatura não desempenha um papel relevante no problema. De fato, a única grandeza termodinâmica importante é a entropia associada ao volume livre disponível para as partículas. Consequentemente, todas as fases e transições de fases são efeitos entrópicos [24]. Num primeiro momento, não parece muito óbvio que a entropia por si só favoreça algum tipo de ordernamento. Para exemplificar o comportamento das fases em sistemas com interações estéricas, vamos discutir dois casos interessantes: esferocilindros e elipsóides de revolução.

Esferocilindros são provavelmente os objetos anisotrópicos mais estudados na literatura 


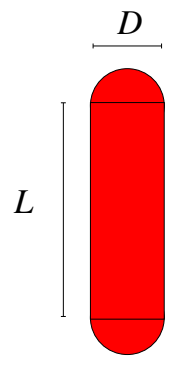

(a)

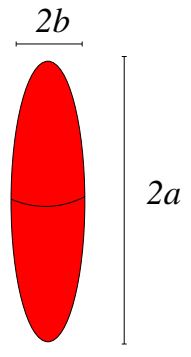

(b)

Figura 4.1: Representação esquemática de objetos anisotrópicos prolatos. (a) esferocilindro de dimensões típicas $L$ e $D$. (b) esferóide com semi-eixos $a$ e $b$.

devido à simplicidade matemática. Um esferocilindro é basicamente um cilindro circular cujas extremidades são capas esféricas, como esquematizado na figura 4.1. (a). Não temos interesse em discutir os diversos tratamentos teóricos associados a tais objetos. Apenas vamos mencionar aspectos gerais do comportamento macroscópico. Para isso, tomemos como exemplo as simulações computacionais desenvolvidas por Bolhuis e Frenkel [53], que obtiveram o diagrama de fases descrito na figura 4.2. Vemos que o sistema apresenta, dada a relação de aspecto $1+L / D$ e a densidade $\rho^{*}$, uma fase isotrópica, mesofases nemática e esmética, e até mesmo fases sólidas de diferentes tipos. Também podemos identificar várias regiões de coexistência. Isso, sem dúvida, é muito interessante, pois as partículas interagem através de potenciais estéricos. Note que no limite $L \rightarrow 0$, teríamos um gás de esferas rígidas, cujo diagrama de fases possivelmente apresenta uma transição sólido-fluido descontínua na região de altas densidades.

Além dos esferocilindros, existe muito interesse em objetos com formas efetivas descritas por elipsóides de revolução, como na figura 4.1.(b). Novamente, vamos apenas mencionar resultados associados ao comportamento das fases, sem discutir as várias abordagens teóricas existentes na literatura. Possivelmente, as simulações de Frenkel e Mulder [54] é um dos trabalhos mais importantes sobre elipsóides rígidos. Nesse trabalho, os autores chegaram ao diagrama de fases esquemático exibido na figura 4.3. Assim como ocorre no caso de esferocilindros, temos fases isotrópica, nemática e sólidas, dadas a relação de aspecto e a densidade de partículas. Todavia, não aparece fase esmética, o que é muito curioso. Ainda no contexto de simulações, Odriozola [55] obtém um diagrama mais rico, especialmente na região envolvendo as fases sólidas, mas não há fases esméticas estáveis. 


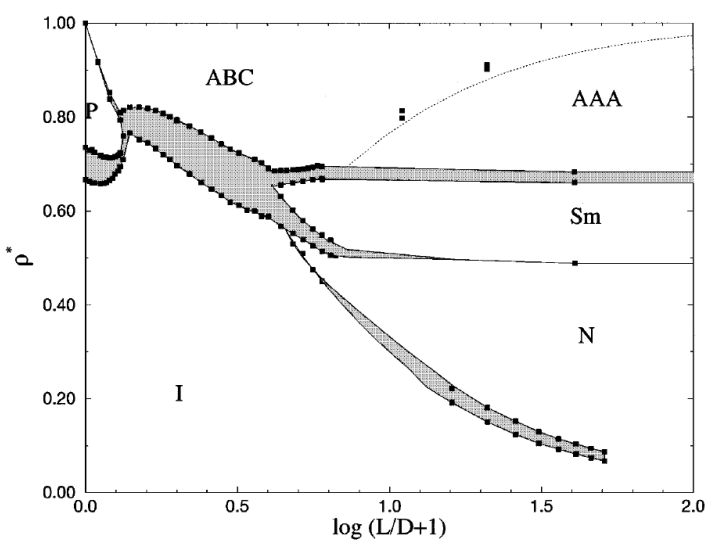

Figura 4.2: Diagrama de fases obtido por Bolhuis e Frenkel para um sistema de esferocilindros rígidos de dimensões típicas $L$ e $D$, onde $\rho^{*}$ é uma densidade reduzida. $I$ : fase isotrópica. $N$ : fase nemática. $S m$ : fase esmética. $P$ : sólido plástico. $A A A$ e $A B C$ : fases sólidas. Regiões hachuradas: coexistência de fases.

De fato, existe um debate interessante sobre a estabilidade de mesofases esméticas em esferóides rígidos [31; 56], e muitos resultados sugerem que tal problemática pode ser superada permitindo deformações na forma esferoidal [56; 57].

A polêmica envolvendo fases esméticas faz parte de uma discussão que tem chamado bastante a atenção: a influência da forma das partículas sobre o comportamento termodinâmico de sistemas com interações estéricas. Entender como essa influência se manisfesta é de enorme interesse para a física de materiais, especialmente no contexto de suspensões coloidais. Por exemplo, a partir de simulações de Monte-Carlo, Damasceno e colaboradores [52] sugerem que objetos com formas efetivas de poliedros convexos têm um comportamento macroscópico rico, com estruturas cristalinas, cristais pláticos e até mesmo cristais líquidos. No caso particular de fases líquido-cristalinas, existe muito interesse nos chamados colóides minerais [28; 29], o que provavelmente se deve à viabilidade de síntese desses materiais.

Do ponto de vista teórico, Onsager [30] desenvolveu um dos primeiros trabalhos com o objetivo de entender o papel da forma das partículas em sistemas coloidais. A teoria de Onsager se baseia nas expansões viriais de um sistema de objetos prolatos rígidos. Como resultado, a energia livre é dada pelo truncamento da série no segundo coeficiente virial. Mais que isso, a energia livre truncada é escrita como um funcional de uma distribuição 


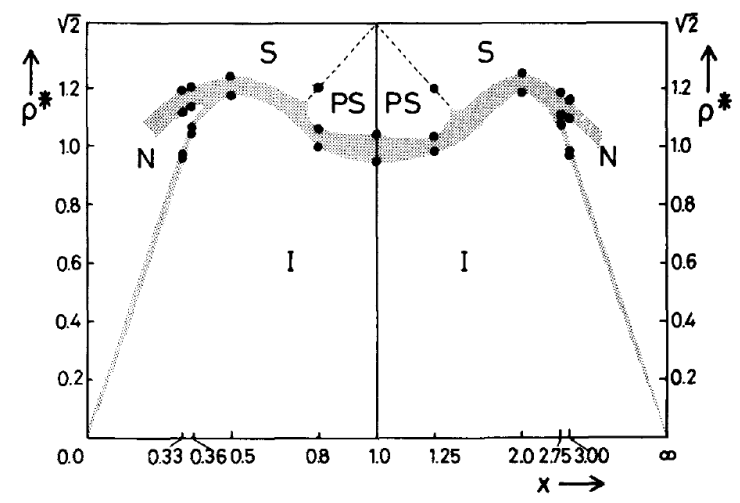

Figura 4.3: Diagrama de fases descrito por Frenkel e Mulder para um sistema de esferóides rígidos de dimensões típicas $2 a$ e $2 b$, onde $\rho^{*}$ é uma densidade reduzida e $x=a / b$ é a relação de aspecto. $I$ : fase isotrópica. $N$ : fase nemática. $P S$ : sólido plástico. $S$ : fase sólida. Regiões hachuradas: coexistência de fases.

associada à probabilidade de haver um objeto com certa orientação. Numa linguagem moderna, podemos dizer que temos uma teoria do funcional densidade para corpos rígidos anisotrópicos [31]. Como veremos na seção 4.2, a teoria de Onsager leva a uma transição nemático-isotrópica de caráter puramente entrópico.

Devido à maneira como é construído, o modelo de Onsager é mais apropriado para descrever sistemas diluídos formados por objetos anisotrópicos muito longos. Isso dificulta bastante o contato com experimentos e simulações, onde os objetos podem ter dimensões características mais realistas. Por exemplo, estimando o terceiro coeficiente virial, Straley [32; 33] conclui que a teoria de Onsager só seria válida quantitativamente para objetos prolatos com relação de aspecto maior que cem. Onsager [30] já apresenta uma discussão para justificar o truncamento da série virial no termo associado ao volume excluído de pares. Certamente, seria interessante desenvolver um modelo simples e que ao mesmo tempo pudesse levar em consideração o tamanho finito dos objetos, bem como densidades finitas. Mostraremos como isso pode ser feito na seção 4.3 .

\subsection{Modelo de "bastões" rígidos}

Nesta seção iremos apresentar o modelo de "bastões" rígidos dentro de uma abordagem heurística na linha da discussão desenvolvida por Sheng [58]. Então, faremos contato 
com o trabalho de Onsager [30], que é basicamente uma teoria do funcional densidade formulada com o auxílio de uma expansão virial truncada no termo referente ao volume excluído de pares.

\subsubsection{Funcional da energia livre}

Vamos considerar um sistema formado por objetos interagentes que têm simetria cilíndrica, onde cada partícula $i$ tem momento e coordenada generalizada $\vec{p}_{i}$ e $\vec{q}_{i}$, respectivamente. É importante lembrar que $\vec{q}$ envolve tanto posição quando orientação. Para um estado particular do sistema, a energia total será da forma

$$
E=\mathcal{K}\left(\vec{p}_{1}, \vec{p}_{2}, \cdots, \vec{p}_{N}\right)+\mathcal{U}\left(\vec{q}_{1}, \vec{q}_{2}, \cdots, \vec{q}_{N}\right)
$$

onde $\mathcal{K}$ é a energia cinética e $\mathcal{U}$ é o potencial de interação entre as partículas. Obviamente, estamos interessados num sistema clássico de muitos corpos. Logo, $\mathcal{K}$ e $\mathcal{U}$ comutam, o que permite escrever a função de partição como

$$
Z=Z_{\text {cin }} Q
$$

onde

$$
Z_{c i n}=\int d \vec{p}_{1} \cdots d \vec{p}_{N} e^{-\beta \mathcal{K}\left(\vec{p}_{1}, \cdots, \vec{p}_{N}\right)},
$$

é a parte de $Z$ relacionada à energia cinética, e

$$
Q=\frac{1}{N !} \int d \vec{q}_{1} \cdots d \vec{q}_{N} e^{-\beta \mathcal{U}\left(\vec{q}_{1}, \cdots, \vec{q}_{N}\right)}
$$

é a parte configuracional, onde incluímos o fator associado à indistiguibilidade das partículas. Além disso, vamos considerar um potencial de pares $u_{i j}=u\left(\vec{q}_{i}, \vec{q}_{j}\right)$ de tal forma que

$$
\mathcal{U}=\sum_{i>j} u_{i j}
$$

O nosso interesse é em sistemas com interações estéricas, de volume excluído, o que nos leva a um potencial de caroço duro

$$
u_{i j}= \begin{cases}\infty, & \text { quando há sobreposição das partículas } \\ 0, & \text { quando não há sobreposição. }\end{cases}
$$


Geralmente, a energia cinética é quadrática nos momentos generalizados, o que resulta num cálculo simples da eq.4.2) em termos de integrais gaussianas. Não é difícil perceber que $Z_{\text {cin }}$ contribui para a energia livre por partícula com termos que dependem apenas da temperatura. Isso significa que podemos focar todo nosso interesse na parte configuracional da função de partição. Mesmo assim, ainda precisamos lançar mão de alguma aproximação. Para exemplificarmos os cálculos que aparecem nesse tipo de problema, seguiremos o trabalho de Onsager [30], na linha de uma expansão virial, dentro de uma abordagem heurística. Vamos começar escrevendo (4.4) como

$$
\begin{aligned}
Q & =\frac{1}{N !} \int d \vec{q}_{1} \cdots d \vec{q}_{N} \prod_{i>j}\left(1-a_{i j}\right), \\
& =\frac{1}{N !} \int d \vec{q}_{1} \cdots d \vec{q}_{N}\left(1-\frac{1}{2} \sum_{i \neq j} a_{i j}+\cdots\right)
\end{aligned}
$$

onde

$$
a_{i j}=1-e^{-\beta u_{i j}}
$$

Um ponto importante consiste em fazer primeiro as integrais sobre as posições, deixando as integrais sobre as orientações para o final. Assim, supondo que a orientação da partícula $i$ é dada pelo vetor unitário $\hat{l}_{i}$, não é difícil mostrar que

$$
Q \simeq \frac{V^{N}}{N !} \int d \hat{l}_{1} \cdots d \hat{l}_{N}\left[1-\frac{1}{2 V} \sum_{i j} V_{e}(i, j)\right]
$$

onde

$$
V_{e}(i, j)=\int d \vec{r}_{i j} a_{i j}
$$

é o volume excluído associado às partículas $i$ e $j, \vec{r}_{i j}$ é o vetor posição relativa e $V$ é o volume do sistema. É importante lembrar que $V_{e}$ é função de $\hat{l}_{i}$ e $\hat{l}_{j}$. Além disso, o volume excluído de pares é definido considerando que as partículas têm orientações fixas.

Para prosseguirmos, vamos adotar a mesma estratégia apresentada por Onsager [30. 41] e supor que existem $N_{\nu}$ partículas com orientação $\hat{l}_{\nu}$, sendo que

$$
N=\sum_{\nu} N_{\nu}
$$

é o número total de partículas do sistema. Aqui, vamos introduzir uma função de distribuição $f\left(\hat{l}_{\nu}\right)$, que é a densidade de probabilidade de haver uma partícula orientada na 
direção $\hat{l}_{\nu}$, o que nos permite escrever

$$
N_{\nu}=\operatorname{Nf}\left(\hat{l}_{\nu}\right) \Delta \hat{l}
$$

onde $\Delta \hat{l}$ é um elemento de ângulo sólido em torno de $\hat{l}_{\nu}$. Dentro desse esquema de discretização, a eq. (4.9) assume a forma

$$
Q \simeq \frac{(\Delta \hat{l})^{N} V^{N}}{N !} \sum_{\left\{N_{\nu}\right\}} \frac{N !}{\prod_{\nu} N_{\nu} !}\left[1-\frac{1}{2 V} \sum_{\nu, \nu^{\prime}} N_{\nu} N_{\nu^{\prime}} V_{e}\left(\nu, \nu^{\prime}\right)\right],
$$

onde $V_{e}\left(\nu, \nu^{\prime}\right)$ é o volume excluído de um par de partículas com orientações $\nu$ e $\nu^{\prime}$, respectivamente. Basicamente, a soma sobre os estados é estimada utilizando a soma sobre os números de ocupação $\left\{N_{\nu}\right\}$.

Nosso interesse é chegar numa expressão válida no limite termodinâmico. Logo, parece razoável estimar a eq. 4.13 usando o termo máximo da soma, especificado por $\left\{\tilde{N}_{\nu}\right\}$. Assim, obtemos a expressão

$$
\begin{aligned}
Q & \simeq \frac{(\Delta \hat{l})^{N} V^{N}}{\prod_{\nu} \tilde{N}_{\nu} !}\left[1-\frac{N^{2}}{2 V} \sum_{\nu, \nu^{\prime}} \frac{\tilde{N}_{\nu}}{N} \frac{\tilde{N}_{\nu^{\prime}}}{N} V_{e}\left(\nu, \nu^{\prime}\right)\right], \\
& \simeq \frac{(\Delta \hat{l})^{N} V^{N}}{\prod_{\nu} \tilde{N}_{\nu} !}\left[1-\frac{\rho}{2} \sum_{\nu, \nu^{\prime}} \frac{\tilde{N}_{\nu}}{N} \frac{\tilde{N}_{\nu^{\prime}}}{N} V_{e}\left(\nu, \nu^{\prime}\right)\right]^{N},
\end{aligned}
$$

onde $\rho=\frac{N}{V}$ é a densidade de partículas. Note que, nessa abordagem, estamos considerando que o sistema está numa situação de baixas densidades. Agora vamos tomar o logaritmo da função de partição e utilizar a aproximação de Stirling, de tal modo que podemos escrever

$$
\begin{aligned}
\ln Q \simeq & N\left\{\ln (\Delta \hat{l})-\ln \rho+1-\sum_{\nu} \frac{\tilde{N}_{\nu}}{N} \ln \frac{\tilde{N}_{\nu}}{N}+\right. \\
& \left.+\ln \left[1-\frac{\rho}{2} \sum_{\nu, \nu^{\prime}} \frac{\tilde{N}_{\nu}}{N} \frac{\tilde{N}_{\nu^{\prime}}}{N} V_{e}\left(\nu, \nu^{\prime}\right)\right]\right\} .
\end{aligned}
$$

Finalmente, de posse da relação 4.12 e tomando o limite do contínuo $\Delta \hat{l} \rightarrow 0$, chegamos à chamada densidade de energia livre para um sistema homogêneo de bastões rígidos,

$$
\begin{aligned}
\mathcal{F}=-\frac{k T}{V} \ln Q= & k T\left[\rho(\ln \rho-1)+\rho \int d \hat{l} f(\hat{l}) \ln f(\hat{l})\right]- \\
& -k T \rho \ln \left[1-\frac{\rho}{2} \int d \hat{l} d \hat{l}^{\prime} f(\hat{l}) f\left(\hat{l}^{\prime}\right) V_{e}\left(\hat{l}, \hat{l}^{\prime}\right)\right],
\end{aligned}
$$


em que usamos a restrição

$$
1=\int d \hat{l} f(\hat{l})
$$

devido à normalização da distribuição orientacional. Uma equação semelhante é obtida por Vertogen e de Jeu [59], seguindo uma aproximação de van der Waals para o volume livre por partícula.

Para fazermos contato com a teoria de Onsager [30], devemos consider que o sistema está no limite de baixas densidades. Isso significa que podemos desenvolver apropriadamente a eq.4.16 em série de potências de $\rho$. Como resultado, obtemos

$$
\begin{aligned}
\mathcal{F}= & k T\left[\rho(\ln \rho-1)+\rho \int d \hat{l} f(\hat{l}) \ln f(\hat{l})+\right. \\
& \left.+\frac{\rho^{2}}{2} \int d \hat{l} d \hat{l}^{\prime} f(\hat{l}) f\left(\hat{l}^{\prime}\right) V_{e}\left(\hat{l}, \hat{l}^{\prime}\right)\right]
\end{aligned}
$$

É importante mencionar que Onsager chega a uma expressão semelhante supondo também que os bastões são muito longos, o que permitiria considerar apenas o volume excluído de pares. Note que optamos por escrever uma densidade de energia livre (energia por volume), pois é a forma mais comum de trabalhar em física de cristais líquidos. Por outro lado, Onsager chega a uma energia livre por partícula, num esquema habitual de física estatística.

\subsubsection{Distribuição estacionária e equações de estado}

Agora devemos encontrar a distribuição orientacional de equilíbrio para darmos continuidade à nossa análise. Vamos começar reescrevendo a eq.4.18 na forma

$$
\frac{\mathcal{F}}{k T}=\rho(\ln \rho-1)+\rho J[f]
$$

em que

$$
\begin{aligned}
J[f]= & \int d \hat{l} f(\hat{l}) \ln f(\hat{l})+\frac{\rho^{2}}{2} \int d \hat{l} d \hat{l}^{\prime} f(\hat{l}) f\left(\hat{l}^{\prime}\right) V_{e}\left(\hat{l}, \hat{l}^{\prime}\right)+ \\
& +\lambda\left(\int d \hat{l} f(\hat{l})-1\right)
\end{aligned}
$$

é um funcional de $f(\hat{l})$, e introduzimos o multiplicador de Lagrange $\lambda$ devido ao vínculo dado pela eq.(4.17). Na prática, temos um problema variacional associado ao funcional 
$J[f]$, cuja distribuição estacionária é obtida resolvendo uma equação de Euler-Lagrange, dada por

$$
\ln f(\hat{l})+1+\lambda+\rho \int d \hat{l}^{\prime} f\left(\hat{l}^{\prime}\right) V_{e}\left(\hat{l}, \hat{l}^{\prime}\right)=0 .
$$

É possível eliminar $\lambda$ usando a condição de normalização de $f(\hat{l})$, o que leva ao resultado

$$
\begin{aligned}
f(\hat{l}) & =\frac{1}{Z} \exp \left[-\rho \int d \hat{l}^{\prime} f\left(\hat{l}^{\prime}\right) V_{e}\left(\hat{l}, \hat{l}^{\prime}\right)\right], \\
Z & =\int d \hat{l} \exp \left[-\rho \int d \hat{l}^{\prime} f\left(\hat{l}^{\prime}\right) V_{e}\left(\hat{l}, \hat{l}^{\prime}\right)\right] .
\end{aligned}
$$

Logo, conhecendo o volume excluído de pares, a distribuição estacionária é dada resolvendo uma equação integral não-linear, sendo que também temos que especificar a densidade $\rho$.

A partir da distribuição 4.22, a densidade de energia livre 4.18 assume a forma

$$
\frac{\mathcal{F}}{k T}=\rho(\ln \rho-1)-\frac{\rho^{2}}{2} \int d \hat{l} d \hat{l}^{\prime} f(\hat{l}) f\left(\hat{l}^{\prime}\right) V_{e}\left(\hat{l}, \hat{l}^{\prime}\right)-\rho \ln Z
$$

É importante mencionar que essa ainda não é a energia livre de equilíbrio, haja vista que chegamos a essa equação usando a expressão associada à distribuição estacionária. Em princípio, devemos encontrar todas as funções $f(\hat{l})$ que são soluções da eq. 4.22 para $\rho$ fixo. De posse dessas soluções, a distribuição de equilíbrio é aquela com menor valor da eq. 4.23 . Outro ponto importante é que, de posse da distribuição $f(\hat{l})$, podemos determinar a média do segundo polinômio de Legendre,

$$
S \equiv\left\langle P_{2}(\cos \theta)\right\rangle=\int d \hat{l} f(\hat{l}) P_{2}(\cos \theta)
$$

onde $\theta$ é o ângulo entre o diretor molecular e o diretor nemático do sistema. Por simplicidade, podemos definir o sistema de referência do laboratório de tal forma que o eixo $z$ seja paralelo ao diretor nemático.

Uma vez que temos a densidade de energia livre, podemos determinar as equações de estado por meio das relações

$$
\begin{aligned}
& P=-\mathcal{F}+\rho \frac{\partial \mathcal{F}}{\partial \rho}, \\
& \mu=\frac{\partial \mathcal{F}}{\partial \rho},
\end{aligned}
$$

onde $P$ é a pressão e $\mu$ é o potencial químico. Note que, do ponto de vista do formalismo termodinâmico, a pressão é a transformada de Legendre de $-\mathcal{F}$, onde a variável independente passa a ser $\mu$. Outro ponto importante é que, em sistemas com interações estéricas, 
geralmente se trabalha com a fração de empacotamento

$$
\eta=\rho v_{0}
$$

onde $v_{0}$ é o volume de uma partícula. Assim, vamos definir um volume excluído adimensional

$$
\overline{V_{e}}\left(\hat{l}, \hat{l}^{\prime}\right)=\frac{V_{e}\left(\hat{l}, \hat{l}^{\prime}\right)}{v_{0}},
$$

o que nos possibilita escrever as equações de estado em termos de uma pressão reduzida

$$
\bar{P} \equiv \frac{P v_{0}}{k T}=\eta+\frac{\eta^{2}}{2} \int d \hat{l} d \hat{l}^{\prime} f(\hat{l}) f\left(\hat{l}^{\prime}\right) \overline{V_{e}}\left(\hat{l}, \hat{l}^{\prime}\right)
$$

e de um potencial químico reduzido

$$
\bar{\mu} \equiv \frac{\mu}{k T}+\ln v_{0}=\ln \eta-\ln Z
$$

Note que, para o caso de um gás de esferas duras, a equação da pressão é consistente com os resultados da expansão virial,

$$
\bar{P} \equiv \frac{P v_{0}}{k T}=\eta+4 \eta^{2}
$$

No caso de num fluido isotrópico formado por moléculas não-esféricas, o segundo coeficiente virial seria dado pela média isotrópica do volume excluído de pares,

$$
B_{2}=\frac{\left\langle V_{e}\right\rangle_{i s o}}{2}
$$

É claro que não é uma tarefa fácil calcular $V_{e}$ para dois objetos anisotrópicos com orientações fixas. Contudo, no caso de corpos rígidos convexos, existe um resultado analítico para a média isotrópica do volume excluído [60; 61], que é dada por

$$
\left\langle V_{e}\right\rangle_{i s o}=2 v_{0}+\frac{S M}{2 \pi}
$$

onde $S$ e $M$ são, respectivamente, a área superficial e a curvatura média de um objeto.

\subsubsection{Transição nemática-isotrópica num sistema de cilindros longos}

Agora precisamos obter uma equação que descreva o volume excluído para um par de objetos convexos prolatos muito longos. Vamos usar como exemplo um sistema formado 


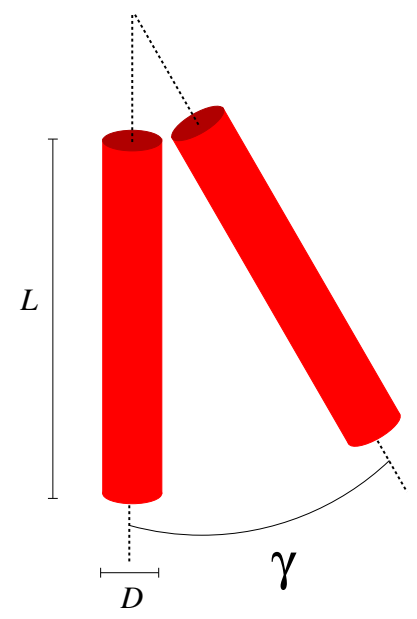

Figura 4.4: Representação de uma configuração de dois cilindros circulares, de tamanhos característicos $L$ e $D$, e com orientação relativa $\gamma$.

por cilindros circulares de comprimento $L$ e diâmetro $D$, como mostrado na figura 4.4 . Onsager [30] chega a uma expressão complicada para o volume excluído de pares. Porém, essa expressão assume uma forma mais simples quando $L \gg D$. Note que essa suposição é consistente com o truncamento da expansão virial no segundo termo. Assim, nesse limite, o volume excluído é dado por

$$
V_{e}^{(c)}=2 D L^{2}|\sin \gamma|
$$

onde $\gamma$ é o ângulo entre os eixos de simetria dos cilindros.

Objetos com formas efetivas de esferocilindros, esquematizados na figura 4.1, também são bastante utilizados para estudos de fases e transições de fases em sistemas de caroço duro. Esses objetos são cilindros de comprimento $L$, com extremidades dadas por capas semi-esféricas de diâmetro $D$. Esferocilindros apresentam uma equação bem simples para o volume excluído de pares [31, dada por

$$
V_{e}^{(e c)}=\frac{4 \pi D^{3}}{3}+2 \pi D^{2} L+2 D L^{2} \sin \gamma
$$

De fato, o traballho de Onsager [30] já apresenta esse resultado. Não é difícil perceber que a eq.4.33 pode ser obtida a partir da eq.4.34 quando os objetos são prolatos e muito longos.

O ponto que queremos destacar é que não estamos interessados nos aspectos quantitativos do problema. Nosso objetivo é estudar a transição entre as fases nemática e isotrópica, que ocorre em esferocilindros, cilindros circulares e esferóides. Assim, parece 
razoável obter uma aproximação para o volume excluído sem que haja perdas qualitativas relevantes. Uma maneira de fazer isso é supondo que possamos expandir $V_{e}^{(c)}$ numa série de Legendre,

$$
V_{e}^{(c)}=a_{0}+a_{2} P_{2}(\cos \gamma)+a_{4} P_{4}(\cos \gamma)+\cdots
$$

onde $a_{i}$ são os coeficientes associados aos polinômios de Legendre $P_{i}$, com $i=0,2,4, \cdots$, por simetria. Então, vamos truncar a série no segundo termo, o que geralmente é chamado de aproximação $P_{2}$, como descrito, por exemplo, no trabalho de Vroege [29].

Para determinar os coeficientes, podemos usar configurações cujos volumes excluídos possam ser estimados sem dificuldade. Configurações razoáveis seriam quando os cilindros são paralelos ou perpendiculares. Assim, considerando $L \gg D$, não é difícil mostrar que

$$
V_{e}^{(c)} \simeq 4 L^{2} D\left[1-P_{2}(\cos \gamma)\right]
$$

Ou seja, levando em consideração apenas o segundo polinômio de Legendre, $V_{e}^{(c)}$ apresenta uma expressão que é matematicamente semelhante ao potencial de interação descrito no modelo de Maier-Saupe. Isso sugere que podemos descrever o estado orientacional do mesógeno através de um tensor do tipo

$$
\boldsymbol{\sigma}=\frac{1}{2}(3 \vec{l} \otimes \vec{l}-\mathbf{1})
$$

onde $\vec{l}$ é o vetor unitário ao longo do eixo de simetria do cilindro, e $\mathbf{1}$ é o tensor identidade. Por fim, o volume excluído para dois cilindros muito longos será dado por

$$
V_{e}^{(c)}=4 L^{2} D\left(1-\frac{2}{3} \boldsymbol{\sigma}_{1} \cdot \boldsymbol{\sigma}_{2}\right)
$$

onde $\boldsymbol{\sigma}_{1} \cdot \boldsymbol{\sigma}_{2} \equiv \operatorname{Tr}\left(\boldsymbol{\sigma}_{1} \boldsymbol{\sigma}_{2}\right)$.

De posse da expressão 4.38), podemos escrever as equações de estado 4.28)-4.29 de forma explícita, o que nos permite fazer o estudo do comportamento das fases isotrópica e nemáticas uniaxiais. Na figura 4.5 mostramos o gráfico $S \times \bar{\eta}$, onde o parâmetro $S$ é dado pela eq. 4.24 e $\bar{\eta}=\kappa \eta$ é uma fração volumétrica reduzida, sendo que $\kappa=\frac{L}{D}$ é a relação de aspecto de um cilindro. Na prática, $\bar{\eta}$ é basicamente o único parâmetro independentẹ no modelo quando $L \gg D$. As linhas pretas indicam as soluções estáveis e as linhas vermelhas são as soluções não-estáveis, que englogam situações metaestáveis e instáveis.

\footnotetext{
${ }^{1}$ Onsager [30] analisa o problema, no ensemble canônico, em termos dessa grandeza.
} 


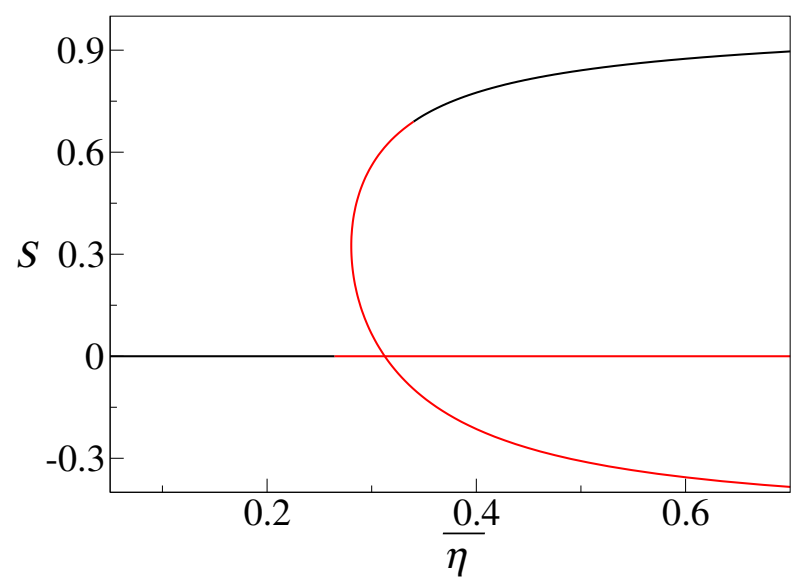

Figura 4.5: Parâmetro $S \times$ fração volumétrica $\bar{\eta}$. Linhas pretas: soluções estáveis. Linhas vermelhas: soluções não-estáveis ( metaestáveis e/ou instáveis).

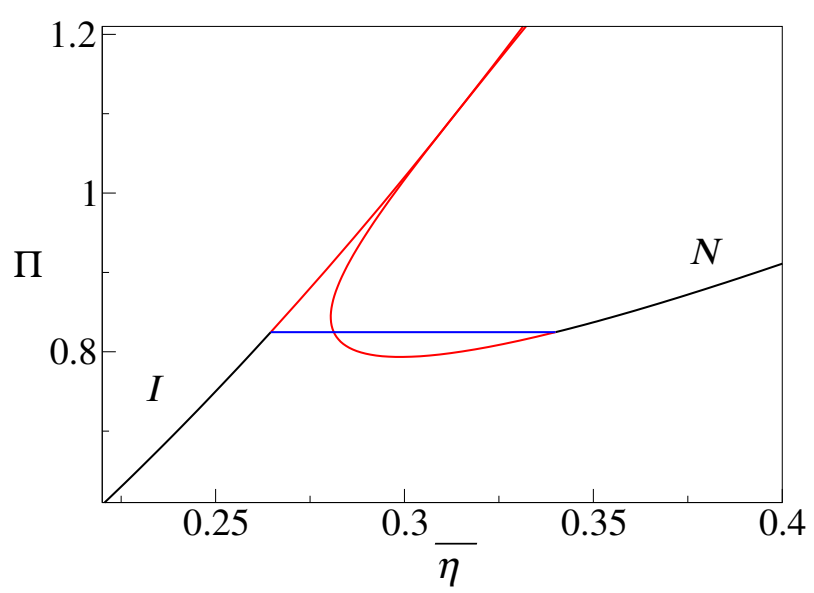

Figura 4.6: Pressão $\Pi \times$ fração volumétrica $\bar{\eta}$. Linhas pretas: soluções estáveis. Linhas vermelhas: soluções não-estáveis ( metaestáveis e/ou instáveis). Linha azul: coexistência de fases. $I$ : fase isotrópica. $N$ : fase nemática.

Claramente, o sistema apresenta um transição nemático-isotrópica de primeira ordem. Para $\bar{\eta}$ grande, existem duas soluções nemáticas uniaxiais: calamítica $(S>0)$ e discótica $(S<0)$. Contudo, apenas a solução calamítica é favorecida termodinamicamente.

Na figura 4.6 exibimos o gráfico $\Pi \times \bar{\eta}$, onde $\Pi=\kappa \bar{P}$ é uma pressão reduzida. Aqui, também fica evidente o caráter descontínuo da transição, que aparece na forma de uma coexistência (linha azul) entre as fases isotrópica e nemática uniaxial. Obviamente, na coexistência, as pressões de $I$ e $N$ devem ser iguais. É importante mencionar que é perfeitamente possível estabelecer uma construção de Maxwell, uma vez que esta é equivalente às condições de equilíbrio. Diferentemente do que o ocorre no modelo de Maier-Saupe, 
a transição nemática-isotrópica num modelo de caroço duro é de natureza puramente entrópica, sem influência da temperatura.

\subsection{Teoria do funcional densidade para nemáticos em altas densidades}

Nesta seção iremos apresentar uma formulação alternativa do problema, discutida no trabalho de Nascimento et al. [62], com o objetivo de estudar a transição nemáticaisotrópica em situações que vão além do regime da teoria de Onsager, que é mais apropriada para baixas densidades. Além disso, também mudaremos o foco para um sistema de esferóides prolatos, que são formas mais sugestivas para descrever moléculas líquidocristalinas.

\subsubsection{Funcional da energia livre}

Assim como fizemos na seção anterior, vamos estudar um sistema de objetos convexos rigídos e prolatos, que interagem através de um potencial do tipo caroço duro 4.6). Cada objeto $i$ do sistema é descrito por uma coordenada generalizada $\vec{q}_{i}$, que representa a posição e a orientação do objeto. Apenas a parte configuracional da função de partição é relevante para o problema, o que nos permite escrever a energia livre configuracional

$$
F=-k T \ln \left\{\frac{1}{N !}\left(\prod_{i=1}^{N} \int d \vec{q}_{i}\right) \exp \left(-\frac{1}{2 k T} \sum_{i \neq j} u_{i j}\right)\right\} .
$$

Para simplificar a análise, vamos introduzir a grandeza

$$
G_{N}=\left(\prod_{i=1}^{N} \int d \vec{q}_{i}\right) \exp \left(-\frac{1}{2 k T} \sum_{i \neq j} u_{i j}\right)
$$

cuja interpretação seria o número de estados disponíveis para $N$ partículas distinguíveis. Agora vamos supor que adicionamos uma partícula ao sistema. Não é difícil perceber que o análogo à eq.4.40 é do tipo

$$
G_{N+1}=\left(\prod_{i=1}^{N} \int d \vec{q}_{i}\right) \exp \left(-\frac{1}{2 k T} \sum_{i \neq j} u_{i j}\right)\left[\int d \vec{q}_{N+1} \exp \left(-\frac{1}{k T} \sum_{i=1}^{N} u_{i(N+1)}\right)\right] \text {. }
$$


Além disso, a probabilidade de encontrar o sistema na configuração $\left(\vec{q}_{1}, \cdots, \vec{q}_{N}\right)$ é dada por

$$
P\left(\vec{q}_{1}, \cdots, \vec{q}_{N}\right)=\frac{1}{G_{N}} \exp \left(-\frac{1}{2 k T} \sum_{i \neq j} u_{i j}\right),
$$

o que nos permite escrever a eq.4.41) como

$$
\begin{aligned}
G_{N+1} & =G_{N}\left\langle\int d \vec{q}_{N+1} \exp \left(-\frac{1}{k T} \sum_{i=1}^{N} u_{i(N+1)}\right)\right\rangle, \\
& =G_{N}\left(\prod_{i=1}^{N} \int d \vec{q}_{i}\right) P\left(\vec{q}_{1}, \cdots, \vec{q}_{N}\right)\left[\int d \vec{q}_{N+1} \exp \left(-\frac{1}{k T} \sum_{i=1}^{N} u_{i(N+1)}\right)\right],
\end{aligned}
$$

onde a média é calculada usando a probabilidade 4.42).

Porém, como todas as partículas do sistema são equivalentes, a eq.4.40 assume a forma

$$
G_{N}=\left\langle\int d \vec{q}_{1} \exp \left(-\frac{1}{k T} \sum_{i \neq 1}^{N} u_{1 i}\right)\right\rangle^{N} .
$$

De fato, a integral basicamente determina o "volume" médio disponível por partícula num ensemble constituído de $N$ partículas. Uma maneira sugestiva de escrever (4.44) é através da expressão

$$
G_{N}=\left\{\int d \vec{q}_{1}\left[1-W\left(\vec{q}_{1}\right)\right]\right\}^{N}
$$

onde

$$
W\left(\vec{q}_{1}\right)=\left\langle 1-\exp \left(-\frac{1}{k T} \sum_{i \neq 1}^{N} u_{1 i}\right)\right\rangle,
$$

pode ser entendido como a média da fração de volume excluído. Consequentemente, a energia livre (4.39) assume a forma

$$
F=-k T \ln \frac{1}{N !}\left\{\int d \vec{q}_{1}\left[1-W\left(\vec{q}_{1}\right)\right]\right\}^{N} .
$$

Note que apenas reescrevemos a energia livre numa forma que explicita os efeitos das interações estéricas. Além disso, do ponto de vista formal, não há problema em admitir que, na eq.4.46), o potencial $u_{i j}$ também possa está associado a forças atrativas.

Dentro de uma perspectiva de campo médio, podemos fazer algumas considerações sobre a eq.4.46), que está associada à fração de volume acessível para uma partícula. Grosso modo, podemos dizer que

$$
W \simeq \frac{N}{V} v_{e f f}
$$


onde $v_{\text {eff }}$ é o volume efetivo ocupado por uma partícula. No caso de esferas rígidas de volume $v_{0}$, o volume excluído por partícula é $4 v_{0}$, de tal forma que, para baixas densidades, podemos escrever

$$
v_{e f f}=4 v_{0}
$$

consistente com a aproximação de van der Waals. Contudo, o volume médio disponível por partícula tende a diminuir à medida que a densidade aumenta, o que pode ser interpretado como uma redução no volume excluído por partícula. De fato, para esferas rígidas, os resultados para o empacotamento denso sugerem que

$$
v_{e f f} \simeq \frac{4}{3} v_{0}
$$

Assim, vamos supor que

$$
W\left(\vec{q}_{1}\right)=\lambda \int d \vec{q}_{2} \rho\left(\vec{q}_{2}\right)\left\{1-\exp \left[-\frac{u\left(\vec{q}_{1}, \vec{q}_{2}\right)}{k T}\right]\right\}
$$

onde $\rho(\vec{q})$ é a função densidade local de partículas que têm coordenada generalizada $\vec{q}$. O parâmetro $\lambda$ é introduzido com o objetivo de fazer contato com as simulações e com os experimentos. Escrita dessa forma, fica mais claro que $W$ determina a fração de volume indisponível, em média, para uma dada partícula devido à presença das demais partículas do sistema.

Agora vamos desenvolver uma teoria do funcional densidade para o nosso problema. Para isso, seguiremos um esquema semelhante ao descrito quando introduzimos as equações 4.11) e 4.12). A diferença é que vamos trabalhar com a densidade de partículas, ao invés de lidar com a distribuição orientacional. Então, considere que a densidade $\rho(\vec{q})$ seja uma função que varia muito lentamente com $\vec{q}$. Também vamos supor que existam $N_{\nu}$ partículas com coordenada generalizada $\overrightarrow{q_{\nu}}$, que basicamente especifica uma região $\nu$ do espaço de fase. Podemos tomar um elemento de volume $\Delta \vec{q}_{\nu}$ suficientemente pequeno em torno de $\vec{q}_{\nu}$, de tal modo que $\rho(\vec{q})$ seja aproximadamente constante. Assim, a energia livre $F_{\nu}$ na região $\nu$ será da forma 4.47, que pode ser escrita como

$$
F_{\nu}=-k T \ln \left\{\frac{1}{\rho\left(\vec{q}_{\nu}\right)}\left[1-W\left(\vec{q}_{\nu}\right)\right]\right\}^{\rho\left(\vec{q}_{\nu}\right) \Delta \vec{q}_{\nu}} .
$$

Por outro lado, a energia livre é uma grandeza aditiva, de tal forma que a energia livre do sistema será dada por

$$
F=k T \sum_{\nu} \Delta \vec{q}_{\nu} \rho\left(\vec{q}_{\nu}\right) \ln \rho\left(\vec{q}_{\nu}\right)-k T \sum_{\nu} \Delta \vec{q}_{\nu} \rho\left(\vec{q}_{\nu}\right) \ln \left[1-W\left(\vec{q}_{\nu}\right)\right]
$$


que, tomando o limite do contínuo $\Delta \vec{q}_{\nu} \rightarrow 0$, leva ao funcional

$$
F=k T \int d \vec{q}_{1} \rho\left(\vec{q}_{1}\right) \ln \rho\left(\vec{q}_{1}\right)-k T \int d \vec{q}_{1} \rho\left(\vec{q}_{1}\right) \ln \left[1-W\left(\vec{q}_{1}\right)\right] .
$$

Nosso objetivo é estudar o comportamento das fases de um ensemble de corpos anisotrópicos com simetria cilíndrica, onde cada objeto do sistema é especificado pela coordenada generalizada $\vec{q}=(\vec{r}, \hat{l})$, onde $\vec{r}$ é o vetor posição e $\hat{l}$ é o vetor unitário associado à orientação. Supondo que o sistema seja translacionalmente homogêneo, é possível escrever a densidade local como

$$
\rho(\vec{q})=\rho_{o} f(\hat{l})
$$

onde $\rho_{o}=\frac{N}{V}$ é simplesmente a densidade de partículas, e $f(\hat{l})$ é uma função de distribuição orientacional que deve satisfazer a condição

$$
\int d \hat{l} f(\hat{l})=1
$$

Logo, partindo dessas considerações, o funcional da energia livre (4.54) assume a forma

$$
\begin{aligned}
F= & k T V\left\{\rho_{o} \ln \rho_{o}+\rho_{o} \int d \hat{l}_{1} f\left(\hat{l}_{1}\right) \ln f\left(\hat{l}_{1}\right)-\right. \\
& \left.-\rho_{o} \int d \hat{l}_{1} f\left(\hat{l}_{1}\right) \ln \left[1-\lambda \rho_{o} \int d \hat{l}_{2} f\left(\hat{l}_{2}\right) V_{e}\left(\hat{l}_{1}, \hat{l}_{2}\right)\right]\right\},
\end{aligned}
$$

onde

$$
V_{e}\left(\hat{l}_{1}, \hat{l}_{2}\right)=\int d \vec{r}_{12}\left(1-e^{-\beta u_{12}}\right)
$$

é o volume excluído para um par de partículas com orientações fixas $\hat{l}_{1}$ e $\hat{l}_{2}$, respectivamente, e $\vec{r}_{12}$ é o vetor posição relativa. É claro que, no limite de baixas densidades, o sistema é descrito por uma energia livre análoga àquela obtida por Onsager [30].

Uma observação importante é que a eq. 4.57) é válida apenas quando o argumento do logaritmo é positivo. Portanto, devemos ter

$$
\lambda \rho_{o} \int d \hat{l}_{2} f\left(\hat{l}_{1}\right) V_{e}\left(\hat{l}_{1}, \hat{l}_{2}\right)<1
$$

Por outro lado, a distribuição orientacional de equilíbrio não é conhecida a priori e depende da densidade $\rho_{o}$. Dentro de um perspectiva heurística, é razoável pensar que as partículas tendem a se alinhar cada vez mais à medida que $\rho_{o}$ aumenta, devido à redução no volume excluído médio. Ou seja, esperamos que a densidade tenha um limite superior, que pode ser estimado como o inverso do menor valor para o volume excluído de 
pares. Assim, parece razoável supor que a função de distribuição orientacional dependa sensivelmente de $\rho_{o}$.

Diferentemente do que fizemos na seção 4.2.1, aqui iremos manter a dependência logarítmica da energia livre 4.57) com o objetivo de estudar o comportamento termodinâmico do sistema para densidades finitas. Isso tem uma consequência muito interessante: a distribuição orientacional de equilíbrio $f(\hat{l})$ é uma função contínua de $\hat{l}$, mas é nula sobre uma região do espaço orientacional. Em outras palavras, existem regiões do espaço de orientações que não são acessíveis a um sistema de objetos anisotrópicos.

\subsubsection{Distribuição estacionária para esferóides prolatos}

Vamos aplicar a teoria até aqui desenvolvida para o caso de esferóides rígidos. Considere um sistema formado por elipsóides de revolução com semieixos maior e menor dados por $L / 2$ e $W / 2$, como mostrado na figura 4.7. O volume de um elipsóide é da forma

$$
v_{0}=\frac{\pi L^{3}}{6 \kappa^{2}}
$$

onde $\kappa=L / W$ é a relação de aspecto. Assim como fizemos na seção 4.2.3, vamos aproximar o volume excluído de pares por uma expansão de Legendre truncada no segundo termo. Ou seja, $V_{e}$ é dado por uma expressão do tipo

$$
\begin{aligned}
& V_{e}=C-\frac{2 D}{3} \boldsymbol{\sigma}_{1} \cdot \boldsymbol{\sigma}_{2}, \\
& \boldsymbol{\sigma}_{i} \equiv \boldsymbol{\sigma}\left(\hat{l}_{i}\right)=\frac{1}{2}\left(3 \hat{l}_{i} \otimes \hat{l}_{i}-\mathbf{1}\right),
\end{aligned}
$$

onde $\hat{l}$ é o vetor unitário ao longo do semieixo maior do elipsoide, e os parâmetros positivos $C$ e $D$ podem ser especificados, por exemplo, determinando configurações cujos volumes excluídos são estimados facilmente. Porém, devido à forma da energia livre (4.57), é mais apropriado absorver o parâmetro $\lambda$ na equação de $V_{e}$, o que leva a

$$
\begin{aligned}
\lambda V_{e} & =c-\frac{2 d}{3} \boldsymbol{\sigma}_{1} \cdot \boldsymbol{\sigma}_{2}, \\
c & =\lambda C, \\
d & =\lambda D .
\end{aligned}
$$

Vale a pena lembrar que não estamos supondo que os elipsóides sejam muito longos, de modo que podemos ir além do regime onde a teoria de Osanger é mais apropriada quantitativamente. 


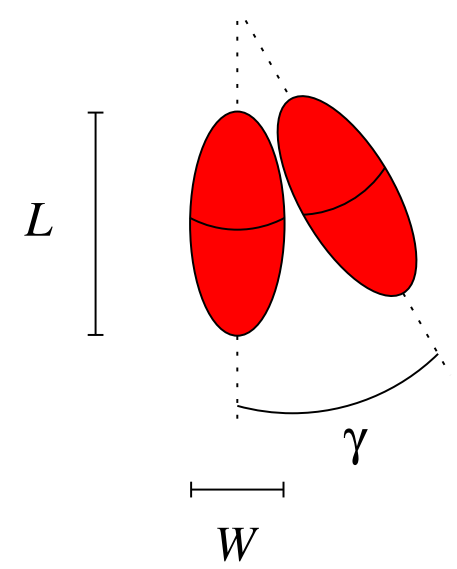

Figura 4.7: Representação esquemática de uma configuração de dois elipsóides idênticos, de tamanhos característicos $L$ e $W$, e com orientação relativa $\gamma$.

Utilizando as equações (4.62) e 4.57), obtemos uma expressão para a densidade de energia livre,

$$
\begin{aligned}
\mathcal{F}= & k T\left\{\rho_{o} \ln \rho_{o}+\rho_{o} \int d \hat{l}_{1} f\left(\hat{l}_{1}\right) \ln f\left(\hat{l}_{1}\right)-\right. \\
& \left.-\rho_{o} \int d \hat{l}_{1} f\left(\hat{l}_{1}\right) \ln \left[1-\rho_{o}\left(c-\frac{2 d}{3} \boldsymbol{\sigma}_{1} \cdot \mathbf{Q}\right)\right]\right\},
\end{aligned}
$$

onde

$$
\mathbf{Q}=\int d \hat{l} f(\hat{l}) \boldsymbol{\sigma}(\hat{l})
$$

é um tensor simétrico de traço nulo que descreve o estado orientacional do sistema. Em outras palavras, $\mathbf{Q}$ é um tensor com simetria quadrupolar. Os autovetores de $\mathbf{Q}$ indicam as direções de ordenamento, e os autovalores dão uma medida do grau de ordenamento ao longo dos eixos principais. Além disso, devemos ter

$$
1-\rho_{o}\left(c-\frac{2 d}{3} \boldsymbol{\sigma}_{1} \cdot \mathbf{Q}\right)>0,
$$

para que a densidade de energia livre seja bem definida.

Agora, vamos introduzir o parâmetro adimensional

$$
\phi=\frac{\rho_{o} c-1}{\rho_{o} d} \in(-\infty, 1]
$$

de tal modo que a densidade é dada por

$$
\rho_{o}=\frac{1}{c-\phi d}
$$


Não é difícil perceber que, no limite de baixas densidades, temos

$$
\rho_{o} \rightarrow 0^{+} \text {quando } \phi \rightarrow-\infty
$$

Por outro lado, no limite de altas densidades,

$$
\rho_{o} \rightarrow \frac{1}{c-d} \text { quando } \phi \rightarrow 1^{-}
$$

que corresponde à densidade máxima de empacotamento do modelo. Logo, em termos de $\phi$, a densidade de energia livre 4.63 pode ser escrita como

$$
\begin{aligned}
\mathcal{F}= & k T \rho_{o}\left[-\ln d+\int d \hat{l}_{1} f\left(\hat{l}_{1}\right) \ln f\left(\hat{l}_{1}\right)-\right. \\
& \left.-\int d \hat{l}_{1} f\left(\hat{l}_{1}\right) \ln \left(\frac{2}{3} \boldsymbol{\sigma}_{1} \cdot \mathbf{Q}-\phi\right)\right],
\end{aligned}
$$

sendo que a eq.4.65 passa a ter uma forma mais simples,

$$
\frac{2}{3} \boldsymbol{\sigma} \cdot \mathrm{Q}-\phi \geq 0
$$

A partir da densidade de energia livre 4.70, temos que seguir os procedimentos típicos de cálculo das variações e determinar a distribuição estacionária, tendo em mente a restrição 4.56). Ou seja, na linha do que discutimos na seção 4.2.2, temos de minimizar um funcional do tipo

$$
\begin{aligned}
J[f]= & \int d \hat{l}_{1} f\left(\hat{l}_{1}\right) \ln f\left(\hat{l}_{1}\right)-\int d \hat{l}_{1} f\left(\hat{l}_{1}\right) \ln \left[\frac{2}{3} \boldsymbol{\sigma}_{1} \cdot \int d \hat{l}_{2} f\left(\hat{l}_{2}\right) \boldsymbol{\sigma}_{2}-\phi\right]+ \\
& +\lambda^{\prime}\left(\int d \hat{l}_{1} f\left(\hat{l}_{1}\right)-1\right)
\end{aligned}
$$

onde $\lambda^{\prime}$ é um multiplicador de Lagrange. Como resultado, é possível mostrar que a distribuição estacionária pode ser escrita como

$$
f(\hat{l})=\frac{1}{Z}\left[\frac{2}{3} \boldsymbol{\sigma}(\hat{l}) \cdot \mathbf{Q}-\phi\right] \exp [\boldsymbol{\sigma}(\hat{l}) \cdot \boldsymbol{\Psi}],
$$

onde

$$
Z=\int d \hat{l}\left[\frac{2}{3} \boldsymbol{\sigma}(\hat{l}) \cdot \mathbf{Q}-\phi\right] \exp [\boldsymbol{\sigma}(\hat{l}) \cdot \mathbf{\Psi}]
$$

sendo que

$$
\boldsymbol{\Psi}=\frac{2}{3} \int d \hat{l}\left[\frac{2}{3} \boldsymbol{\sigma}(\hat{l}) \cdot \mathbf{Q}-\phi\right]^{-1} f(\hat{l}) \boldsymbol{\sigma}(\hat{l})
$$

é um parâmetro tensorial auxiliar. A condição dada pela eq.4.71 implica que a distribuição $f(\hat{l})$ deve ser nula em algumas regiões do espaço orientacional. 


\subsubsection{Equações de estado e transição nemático-isotrópica}

Sabemos que um sistema de uma única componente, formado por moléculas com simetria cilíndrica, exibe apenas fases nemáticas uniaxiais além da fase isotrópica [1] na ausência de campos externos. Logo, iremos focar nossa atenção nas fases uniaxiais, de tal modo que vamos supor que a eq.4.64 é do tipo

$$
\mathbf{Q}=\frac{S}{2}(3 \hat{N} \otimes \hat{N}-\mathbf{1})
$$

onde $S$ é um parâmetro que mede o grau de ordenamento do sistema, e $\hat{N}$ é o vetor unitário diretor nemático, associado à direção da orientação média dos mesógenos. É possível mostrar que $\boldsymbol{\Psi}$ também é um tensor uniaxial e que tem o mesmo sistema de eixos principais de $\mathbf{Q}$, o que leva a

$$
\Psi=\Psi\left(\hat{N} \otimes \hat{N}-\frac{1}{3} \mathbf{1}\right)
$$

Dentro dessa premissa de uniaxialidade, a densidade de energia livre 4.70 pode ser reescrita como

$$
\begin{aligned}
\frac{\mathcal{F}}{k T} & =-\rho_{o} \ln d+\rho_{o} \Psi S-\rho_{o} \ln Z, \\
Z & =\int d x\left[S P_{2}(x)-\phi\right] \exp \left[\Psi P_{2}(x)\right],
\end{aligned}
$$

onde $x=\hat{l} \cdot \hat{N}$ e $P_{2}(x)$ é o segundo polinômio de Legendre. Por outro lado, a distribuição estacionária 4.73 será dada por

$$
f(x)=\frac{1}{Z}\left[S P_{2}(x)-\phi\right] \exp \left[\Psi P_{2}(x)\right]
$$

Note que as integrais que aparecem na descrição do problema devem ser calculadas levando em consideração que $S P_{2}(x)-\phi>0$. Ou seja, para garantir que a função de distribuição seja não-negativa, temos que impor a condição $f(x)=0$ para $S P_{2}(x)-\phi<0$.

Devido à forma truncada do volume excluído, não precisamos determinar $f(x)$ numericamente, pois podemos descrever o sistema através de duas equações acopladas,

$$
\begin{aligned}
& S=\frac{1}{Z} \int d x P_{2}(x)\left[S P_{2}(x)-\phi\right] \exp \left[\Psi P_{2}(x)\right], \\
& \Psi=\frac{1}{Z} \int d x P_{2}(x) \exp \left[\Psi P_{2}(x)\right] .
\end{aligned}
$$


Assim, os limites de integração seriam dados, de forma explícita, por

$$
\begin{aligned}
& \left(x_{0}, 1\right), \text { quando } S>0 \\
& \left(0, x_{0}\right), \text { quando } S<0 .
\end{aligned}
$$

onde $x_{0}$ é obtido resolvendo a equação $S P_{2}\left(x_{0}\right)=\phi$. Na prática, primeiro supomos um certo valor de $\Psi \in(-\infty, \infty)$, o que permite encontrar $x_{0}$ numericamente. Então, tendo em vista que $\phi / S=P_{2}\left(x_{0}\right), S$ pode ser determinado a partir de 4.80 . Por fim, $\phi$ é dado usando a identidade $\phi=S P_{2}\left(x_{0}\right)$. Como resultado, é possível associar $S$ e $\phi$ a cada valor de $\Psi$.

Para obtermos as equações de estado, lançamos mão das expressões 4.25, o que nos leva à pressão adimensional,

$$
\Pi \equiv \frac{P d}{k T}=\frac{1}{Z} \int d x \exp \left[\Psi P_{2}(x)\right]
$$

e ao potencial químico adimensional

$$
\mu^{*} \equiv-\ln d+S \Psi+\frac{1}{Z d} \int d x \exp \left[\Psi P_{2}(x)\right]-\ln Z
$$

É fácil mostrar que, no limite $S \rightarrow 0$, obtemos

$$
\frac{P_{i s o}}{k T}=\frac{\rho_{o}}{1-\rho_{o} c},
$$

que é basicamente a equação de van der Waals para um sistema de objetos anisotrópicos rígidos numa fase isotrópica. Note que podemos identificar $c$ com o segundo coeficiente virial.

Na figura 4.8 mostramos o gráfico do parâmetro $S$ contra $\phi$. As linhas pretas são as soluções estáveis, as linhas verdes e vermelhas representam as soluções metaestáveis e instáveis, respectivamente. Como esperado, vemos que $S \rightarrow 1$ quando $\phi \rightarrow \infty$, o que corresponde à situação onde todos os esferóides estão ordenados paralelamente. Ou seja, para altas densidades, somente a fase uniaxial calamítica $(S>0)$ é termodinamicamente estável. Note que o ramo uniaxial discótico $(S<0)$ torna-se localmente estável à medida que $\phi$ aumenta. A solução isotrópica $(S=0)$ também é metaestável para altas densidades, mas é globalmente estável quando o sistema fica mais diluído. É interessante notar que os estados que não são estáveis têm um comportamento bem peculiar; em especial, a 


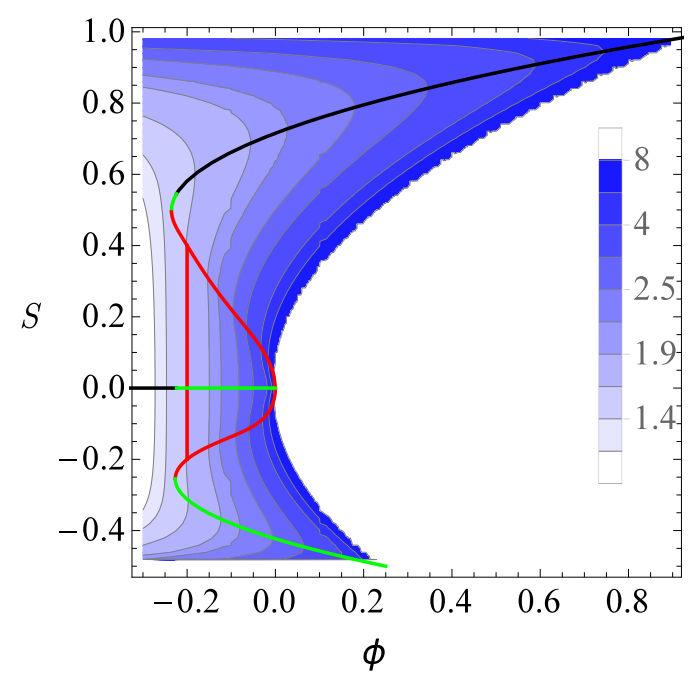

Figura 4.8: $S \times \phi$ para soluções uniaxiais. Linhas pretas: soluções estáveis. Linhas verdes: soluções metaestáveis. Linhas vermelhas: soluções instáveis.

solução instável, representada pela linha vertical vermelha em $\phi=-0.2$, para a qual $-0.2<S<0.4$. Esse tipo de comportamento não é observado no modelo de Onsager, como mostrado na figura 4.5 .

O sistema apresenta uma transição nemático-isotrópica de primeira ordem. Na figura 4.9. (a) exibimos o gráfico $\mathcal{F} / \rho_{o} \times \phi$, onde a grandeza $\mathcal{F} / \rho_{o}$ é simplesmente a energia livre de Helmholtz por partícula. Nesse gráfico, vemos que a transição é descrita pela tangente dupla; a condição de equilíbrio de pressões iguais é dada pela tangente comum, sendo que o ponto onde a tangente cruza o eixo vertical está associado ao potencial químico. De forma equivalente, é possível usar uma construção de Maxwell para estudar a transição, como mostrado em na figura 4.9(b). Nesse caso, a linha azul indica a pressão na coexistência, de tal forma que a linha preta tracejada determina as regiões cujas áreas devem ser iguais. Não é difícil mostrar a equivalência entre as condições de equilíbrio, a construção da tangente dupla e a construção de Maxwell. Em qualquer um desses cenários, é possível concluir que o sistema apresenta uma separação de fases para a qual $\phi_{I}=-0.365$ e $\phi_{N}=0.163$, independentemente dos valores da relação de aspecto. 


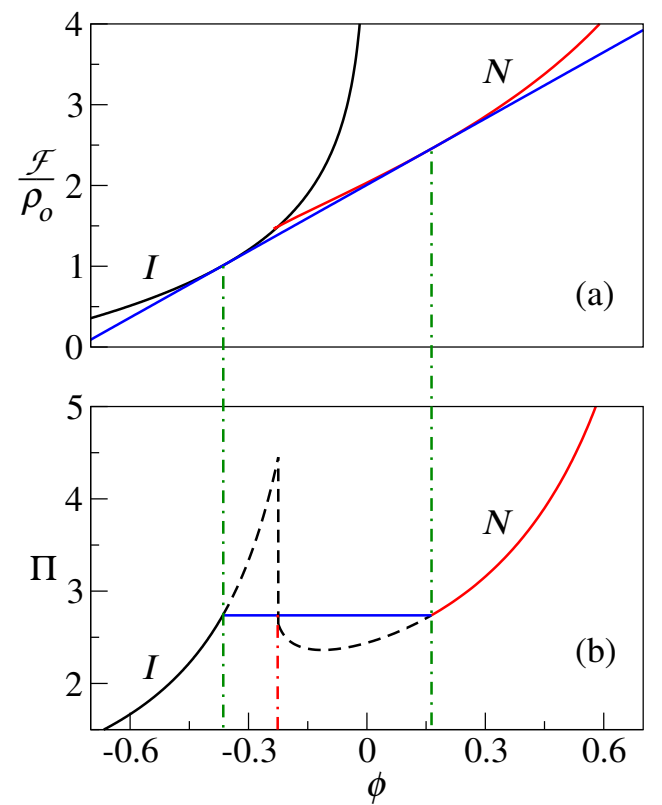

Figura 4.9: Coexistência de fases. (a) Tangente dupla. (b) Construção de Maxwell. I: fase isotrópica. $N$ : fase nemática 


\section{Capítulo 5}

\section{Conclusões e Perspectivas}

Nosso objetivo nesta tese foi estudar modelos microscópicos que possam descrever fases nemáticas de cristais líquidos. Grosso modo, investigamos dois tipos de modelos, que diferem quanto à natureza das interações. Começamos com potenciais de pares associados a forças atrativas, cuja situação mais simples envolve a energia de dispersão de London. Esse tipo de interação é a base para o entendimento do modelo de Maier-Saupe, que é um dos modelos mais importantes para descrever fases nemáticas. Então, passamos para os sistemas de caroço duro, onde um dos trabalhos mais importante foi desenvolvendo por Onsager, com o conhecido modelo de bastões rígidos. Esse modelo, na prática, é formulado no contexto das teorias do funcional densidade, muito importantes para a física de fluidos.

No contexto de forças atrativas, discutimos o modelo de Maier-Saupe para mesógenos intrinsecamente biaxiais. O diagrama de fases apresenta estruturas uniaxiais calamíticas e discóticas, além das fases isotrópica e biaxial. Também encontramos um ponto multicrítico especial denominado ponto de Landau. Em seguida, estudamos o potencial de pares mais geral descrito por Straley, que foi posteriormente reformulado por Sonnet, Virga e Durand. Esse modelo leva a diagrama de fases com topologias distintas, exibindo, por exemplo, pontos triplos e tricríticos. Todos os modelos de objetos biaxiais foram investigados dentro do esquema de discretização das orientações proposto por Zwanzig.

Ainda na linha de interações atrativas, introduzimos um modelo para uma mistura binária de objetos uniaxiais e biaxiais. Nosso objetivo com esse modelo é estudar como as variações de forma dos mesógenos influenciam o diagrama de fases. Adotamos a simplificação de que os objetos se orientam nas direções dos eixos cartesianos, além de tratar a mistura no limite annealed. Assim, numa versão de campo médio, conseguimos obter 
expressões analíticas, tanto do funcional da energia livre quanto das equações de campo médio. Também fizemos contato com as teorias fenomenológicas de Landau-de Gennes, desenvolvendo uma expansão do funcional da energia livre em termos dos invariantes do parâmetro de ordem. Mostramos que o modelo de mistura possui diagramas de fases com topologias muito ricas, exibindo fases reentrantes, pontos de Landau e uma grande variedade de pontos multicríticos (críticos, tricríticos, críticos terminais, etc).

No caso de interações estéricas, estudamos uma teoria do funcional densidade para sistemas anisotrópicos, construída a partir de uma aproximação de campo médio. Nosso interesse foi obter um modelo que fosse capaz de descrever fases nemáticas em altas densidades. Vimos que a distribuição orientacional estacionária pode assumir valores nulos em certas regiões do espaço de orientações. Além disso, o sistema exibe um comportamento "de não-equilíbrio" muito interessante, diferente do obtido pelo modelo de bastões rígidos descrito pela teoria de Onsager. Por fim, mostramos que o modelo leva a uma transição descontínua nemática-isotrópica, caracterizada por uma região de coexistência de fases.

Nesta tese investigamos como as variações de forma dos mesógenos podem influcenciar o diagrama de fases de sistemas líquido-cristalinos. Isso foi feito através de uma mistura binária de objetos intrinsecamente uniaxiais e objetos intrinsecamente biaxiais. Todavia, temos interesse em seguir uma estratégia alternativa e simular as mudanças na forma dos objetos com o auxílio de graus de liberdade elásticos. Um modelo desse tipo pode ser interessante para descrever, por exemplo, o comportamento reentrante da fase isotrópica no diagrama encontrado por Yu e Saupe [8].

Além disso, vale a pena lembrar que estudados a mistura através de uma aproximação de campo médio, onde as flutuações não são levadas em consideração. Logo, é importante desenvolver abordagens que incluam efeitos de coordenação finita, tais como cálculos numa árvore de Cayley ou simulações de Monte Carlo. Isso permitiria verificar como as flutuações poderiam modificar o diagrama de fases.

Para sistemas anisotrópicos rígidos, seria interessante estudar, dentro do modelo que introduzimos, fases nemáticas biaxiais e estruturas esméticas. Outra proposta de trabalho, que pretendemos desenvolver, é uma teoria do funcional densidade que combine forças atrativas e repulsivas. Um tratamento desse tipo poderia levar a um fluido, do tipo van der Waals, com fases líquida e vapor, além de mesofase nemática. 


\section{Referências Bibliográficas}

[1] P. de Gennes and J. Prost, The Physics of Liquid Crystals (Clarendon Press, 2005).

[2] S. Singh, Liquid Crystals, Fundamentals (World Scientific, 2002).

[3] P. Palffy-Muhoray, Phys. Today 60, 54 (2007).

[4] A. M. F. Neto and S. R. Salinas, The Physics of Lyotropic Liquid Crystals (Oxford University Press, 1993).

[5] G. R. Luckhurst, Thin Solids Films 393, 40 (2001).

[6] M. J. Freiser, Phys. Rev. Lett. 24, 1041 (1970).

[7] M. J. Freiser, Mol. Cryst. Liq. Cryst. 14, 165 (1971).

[8] L. J. Yu and A. Saupe, Phys. Rev. Lett. 40, 1000 (1980).

[9] E. Akpinar, D. Reis, and A. M. F. Neto, Eur. Phys. J. E. 35, 50 (2012).

[10] E. Akpinar, D. Reis, and A. M. F. Neto, Liq. Cryst. 39, 881 (2012).

[11] G. R. Luckhurst and T. J. Sluckin, eds., Biaxial Nematic Liquid Crystals: Theory, Simulation and Experiment (Wiley, 2015).

[12] L. A. Madsen, T. J. Dingemans, M. Nakata, and E. T. Samulski, Phys. Rev. Lett. 92, 145505 (2004).

[13] B. R. Acharya, A. Primak, and S. Kumar, Phys. Rev. Lett. 92, 145506 (2004).

[14] K. Merkel, A. Kocot, J. K. Vij, R. Korlacki, G. H. Mehl, and T. Meyer, Phys. Rev. Lett. 93, 237801 (2004). 
[15] J. P. Straley, Phys. Rev. A 10, 1881 (1974).

[16] A. M. Sonnet, E. G. Virga, and G. E. Durand, Phys. Rev. E 67, 061701 (2003).

[17] R. Alben, J . Chem. Phys. 59, 4299 (1973).

[18] P. Palffy-Muhoray and J. R. de Bruyn, J. Chem. Phys. 82, 5294 (1985).

[19] S. R. Sharma, P. Palffy-Muhoray, B. Bergersen, and D. A. Dunmur, Phys. Rev. A 32, 3752 (1985).

[20] E. F. Henriques and V. B. Henriques, J. Chem. Phys. 107, 9036 (1997).

[21] E. do Carmo, D. B. Liarte, and S. R. Salinas, Phys. Rev. E 81, 062701 (2010).

[22] L. Longa, G. Pajak, and T. Wydro, Phys. Rev. E 76, 062503 (2007).

[23] S. Belli, A. Patti, M. Dijkstra, and R. van Roij, Phys. Rev. Lett. 107, 148303 (2011).

[24] D. Frenkel, Nat. Materials 14, 9 (2015).

[25] F. M. van der Kooij and H. N. W. Lekkerkerker, J. Phys. Chem. B 102, 7829 (1998),

[26] A. Kuijk, D. V. Buelov, A. V. Petukhov, A. van Blaaderen, and A. Imhof, Faraday Discussions 159, 181 (2012).

[27] B. J. Lemaire, P. Davidson, J. Ferre, J. P. Jamet, P. Panine, I. Dozov, and J. P. Jolivet, Phys. Rev. Lett. 88, 125507 (2002).

[28] H. N. W. Lekkerkerker and G. J. Vroege, Phil. Trans. R. Soc. A 371, 20120263 $(2013)$.

[29] G. J. Vroege, Liq. Cryst. 41, 342 (2013).

[30] L. Onsager, Ann. NY Acad. Sci. 51, 627 (1949).

[31] L. Mederos, E. Velasco, and Y. Martines-Raton, J. Phys.: Condens. Matter 26, 463101 (2014).

[32] J. P. Straley, Mol. Cryst. Liq. Cryst. 22, 333 (1973). 
[33] J. P. Straley, Mol. Cryst. Liq. Cryst. 24, 7 (1973).

[34] A. D. Buckingham, Adv. Chem. Phys. 12, 102 (1967).

[35] W. J. Goossens, Mol. Cryst. and Liq. Cryst. 12, 237 (1971).

[36] B. W. van der Meer, G. Vertogen, A. J. Dekker, and J. G. J. Ypma, J. Chem. Phys. 65, 3935 (1976).

[37] C. Cohen-Tannoudji, B. Diu, and F. Laloe, Quantum Mechanics, Vol. 2 (Wiley, 1992).

[38] P. M. Chaikin and T. C. Lubensky, Principles of Condensed Matter Physics (Cambridge University Press, 1995).

[39] P. Atkins and R. Friedman, Molecular Quantum Mechanics, 4th ed. (Oxford University Press, 2005).

[40] P. Palffy-Muhoray and X. Zheng, Discrete and Continuous Dynamical Systems, Series B 15, 465 (2011).

[41] R. Zwanzig, J. Chem. Phys. 39, 1714 (1963).

[42] R. Zwanzig, J. Chem. Phys. 24, 855 (1956).

[43] N. Boccara, R. Mejdani, and L. D. Seze, J. Phys. 38, 149 (1977).

[44] H. Goldstein, Classical Mechanics (Addison-Wesley, 1980).

[45] R. A. Sauerwein and M. J. de Oliveira, J. Chem. Phys. 144, 194904 (2016).

[46] E. F. Gramsbergen, L. Longa, and W. H. de Jeu, Phys. Rep. 135, 195 (1986).

[47] E. S. Nascimento, A. P. Vieira, and S. R. Salinas, Braz. J. Phys. 46, 664 (2016).

[48] G. D. Matteis, A. M. Sonnet, and E. G. Virga, Continuum Mech. Thermodyn. 20, 347 (2008).

[49] E. S. Nascimento, E. F. Henriques, A. P. Vieira, and S. R. Salinas, Phys. Rev. E 92, $062503(2015)$. 
[50] L. Q. Amaral, O. R. Santos, W. S. Braga, N. M. Kimura, and A. J. Palangana, Liq. Cryst. 42, 240 (2015).

[51] L. Q. Amaral, Liq. Cryst. 37, 627 (2010).

[52] P. F. Damasceno, M. Engel, and S. C. Glotzer, Science 27, 453 (2012).

[53] P. Bolhuis and D. Frenkel, J. Chem. Phys. 106, 666 (1997).

[54] D. Frenkel and B. Mulder, Mol. Phys. 55, 1171 (1985).

[55] G. Odriozola, J. Chem. Phys. 136, 134505 (2012).

[56] E. G, Mol. Phys. 76, 1359 (1992).

[57] Y. Martines-Raton and E. Velasco, J. Chem. Phys. 129, 054907 (2008).

[58] E. B. Priestley, P. J. Wojtowicz, and P. Sheng, Introduction to Liquid Crystals (Springer, 1974).

[59] G. Vertogen and W. H. de Jeu, Thermotropic Liquid Crystals, Fundamentals (Springer Series in Chem. Phys, 1988).

[60] T. Kihara, Rev. Mod. Phys. 25, 831 (1953).

[61] M. Piastra and E. G. Virga, Phys. Rev. E 91, 062503 (2015).

[62] E. S. Nascimento, P. Palffy-Muhoray, J. M. Taylor, and E. G. Virga, Phys. Rev. E 96, 022704 (2017).

[63] R. Rosso, Liq. Cryst. 34, 737 (2007).

[64] M. Osipov and S. Pikin, J. Phys. II France 34, 1223 (1995).

[65] A. Sonnet and E. Virga, Phys. Rev. E 77, 031704 (2008). 


\section{Representação irredutível de um tensor simétrico}

Considere um tensor simétrico A que representa uma certa grandeza molecular (polarizabilidade elétrica, o tensor de inércia, etc.). Vamos supor que A tenha uma decomposição espectral do tipo

$$
\mathbf{A}=\sum_{i} a_{i} \vec{n}_{i} \otimes \vec{n}_{i}
$$

onde $a_{i}(i=1,2,3)$ é o autovalor de $\mathbf{A}$ associado ao autovetor $\vec{n}_{i}$. Em princípio, os autovetores podem ser todos diferentes. Isso permitiria descrever moléculas que não têm simetria cilíndrica.

Podemos expandir A na forma

$$
\begin{aligned}
\mathbf{A} & =\mathbf{A}_{i}+\mathbf{A}_{a}, \\
\mathbf{A}_{i} & =\frac{\operatorname{Tr}(\mathbf{A})}{3} \mathbf{1} \\
\mathbf{A}_{a} & =\mathbf{A}-\frac{\operatorname{Tr}(\mathbf{A})}{3} \mathbf{1},
\end{aligned}
$$

onde $\mathbf{A}_{i}$ e $\mathbf{A}_{a}$ são, respectivamente, as partes isotrópica e anisotrópica de $\mathbf{A}$, e do tensor identidade 1. $\mathbf{A}_{a}$ estaria associada a desvios da simetria esférica contidos em A. De posse da decomposição espectral A.1, é possível escrever a parte anisotrópica como

$$
\mathbf{A}_{a}=\frac{1}{3} \sum_{i} \Lambda_{i} \vec{n}_{i} \otimes \vec{n}_{i}
$$


com

$$
\begin{aligned}
& \Lambda_{1}=2 a_{1}-a_{2}-a_{3}, \\
& \Lambda_{2}=2 a_{2}-a_{3}-a_{1}, \\
& \Lambda_{3}=2 a_{3}-a_{1}-a_{2},
\end{aligned}
$$

de modo que $\mathbf{A}_{a}$ é dada em termos dos autovetores e autovalores de $\mathbf{A}$.

Agora vamos seguir uma abordagem na linha do trabalho de Rosso [63] bem como Osipov e Pikin [64] e introduzir os tensores de quadrupolo

$$
\begin{aligned}
& \mathbf{q}=\vec{n}_{1} \otimes \vec{n}_{1}-\frac{1}{3} \mathbf{1}, \\
& \mathbf{b}=\vec{n}_{2} \otimes \vec{n}_{2}-\vec{n}_{3} \otimes \vec{n}_{3},
\end{aligned}
$$

construídos a partir dos autovetores $\vec{n}_{i}(i=1,2,3)$. A ideia é que $\mathbf{q}$ e $\mathbf{b}$ formam uma base para o espaço vetorial dos tensores simétricos de traço nulo que podem representar moléculas intrinsecamente biaxiais. Logo, é perfeitamente possível escrever $\mathbf{A}_{a}$ como uma combinação linear dos tensores A.5,

$$
\mathbf{A}_{a}=c_{1} \mathbf{q}+c_{2} \mathbf{b}
$$

onde $c_{1}$ e $c_{2}$ são constantes. De fato, essas constantes podem ser determinadas com o auxílio da eq. A.3. Então obtemos

$$
\begin{aligned}
c_{1} & =a_{1}-\frac{1}{2}\left(a_{2}+a_{3}\right) \\
c_{2} & =\frac{1}{2}\left(a_{2}-a_{3}\right),
\end{aligned}
$$

onde estamos admitindo que $a_{1}$ é o maior autovalor de $\mathbf{A}$.

Seguindo um raciocínio análogo ao que discutimos, Osipov e Pikin [64] expressam a parte anisotrópica da susceptibidade magnética através de uma conbinação linear de tensores simétricos com traço nulo. Uma decomposição semelhante também é mostrada, por exemplo, no trabalho de Sonnet e Virga [65], que expandem a polarizabilidade elétrica com uma superposição dos tensores $\mathbf{1}, \mathbf{q}$ e $\mathbf{b}$. 
APÊNDICE B

\section{Publicações}

A seguir estão as publicações que são fruto deste trabalho. 


\title{
Lattice Statistical Models for the Nematic Transitions in Liquid-Crystalline Systems
}

\author{
E. S. Nascimento ${ }^{1}$ • A. P. Vieira ${ }^{1}$ - S. R. Salinas ${ }^{1}$ (iD
}

Received: 14 July 2016 / Published online: 25 August 2016

C) Sociedade Brasileira de Física 2016

\begin{abstract}
We investigate the connections between some simple Maier-Saupe lattice models, with a discrete choice of orientations of the microscopic directors, and a recent proposal of a two-tensor formalism to describe the phase diagrams of nematic liquid-crystalline systems. This twotensor proposal is used to formulate the statistical problem in terms of fully connected lattice Hamiltonians, with the local nematic directors restricted to the Cartesian axes. Depending on the choice of interaction parameters, we regain all of the main features of the original mean-field two-tensor calculations. With a standard choice of parameters, we obtain the well-known sequence of isotropic, uniaxial, and biaxial nematic structures, with a Landau multicritical point. With another suitably chosen set of parameters, we obtain two tricritical points, according to some recent predictions of the two-tensor calculations. The simple statistical lattice models are quite easy to work with, for all values of parameters, and the present calculations can be carried out beyond the mean-field level.
\end{abstract}

Keywords Liquid crystals $\cdot$ Nematic transitions $\cdot$ Biaxial nematic transitions $\cdot$ Maier-Saupe models

\section{Introduction}

In a number of recent investigations of the phase diagrams of nematic liquid-crystalline systems, with a view to

S. R. Salinas ssalinas@if.usp.br

1 Instituto de Física, Universidade de São Paulo, São Paulo, SP 05508-090, Brazil characterizing biaxial nematic structures, we have introduced some simple lattice models with Maier-Saupe pair interactions [1-4]. We claim that these schematic statistical lattice models, with a restrict choice of orientational variables, and adequately chosen interaction parameters, are already able to account for the qualitative features of the complex phase diagrams of nematic systems. Also, they are amenable to relatively easy statistical mechanics calculations, for a wide choice of parameters, and these calculations may be carried out beyond a mean-field scenario [2]. We were then motivated to investigate the connections of these statistical formulations with some proposals of a quite general two-tensor formalism, at the mean-field level, which describes the classical isotropic-uniaxial-biaxial sequence of phases in the nematic systems as well as the onset of a tricritical point for a particular choice of interaction parameters $[5,6]$.

We first review the definitions of the "order parameters" and the general form of interaction energy used by Sonnet, Virga, and Durand [5]. This SVD interaction reproduces an early proposal of Straley [7] for the most general form of the interaction between a pair of nematogenic units. In Section 2, we use this pair interaction to introduce a fully connected lattice model, with a restriction of the microscopic nematic directors to point along the Cartesian axis, which is reminiscent of an old proposal of Zwanzig to treat the Onsager model of rigid cylinders [8, 9]. If we adopt this Zwanzig suppression of microscopic fluctuations, the statistical problem is considerably simplified. In Section 3, we describe some particular cases. For the simplest choice of interaction parameters, which corresponds to the so-called MSZ model, we regain the weak first-order transition between the isotropic and the uniaxial nematic phases $[2,10]$. The inclusion of a special form of extra (intrinsically biaxial) interactions, which has been 
used by most investigators in this area, leads to the usual isotropic-uniaxial-biaxial sequence, and to a Landau multicritical point [4]. With this choice of parameters, we define an MSZ6 model, which is equivalent to a lattice statistical model originally proposed by Boccara, Medjani, and de Sèze [11]. Although it is not our aim in the present paper to offer a thorough review of the literature, present results are in qualitative agreement with earlier numerical calculations for lattice systems of non-cylindrically symmetric particles $[12,13]$. With another particular choice of energy parameters, we do confirm the existence of a pair of tricritical points, according to the predictions of Matteis and Virga [6].

\section{The Two-Tensor Order Parameter}

According to Sonnet, Virga, and Durand [5], the usual molecular nematic tensor $\mathbf{q}$ is given by the $3 \times 3$ matrix,

$\mathbf{q}=\vec{n}_{1} \otimes \vec{n}_{1}-\frac{1}{3} \mathbf{I}$,

where $\mathbf{I}$ is a unit matrix, the symbol $\otimes$ indicates a tensor product, and $\vec{n}_{1}$, with $\left|\vec{n}_{1}\right|=1$, is the director associated with a uniaxial nematogenic molecule. In a more explicit notation, we write

$\mathbf{q}=\left(\begin{array}{ccc}n_{1 x} n_{1 x}-\frac{1}{3} & n_{1 x} n_{1 y} & n_{1 x} n_{1 z} \\ n_{1 y} n_{1 x} & n_{1 y} n_{1 y}-\frac{1}{3} & n_{1 y} n_{1 z} \\ n_{1 z} n_{1 x} & n_{1 z} n_{1 y} & n_{1 z} n_{1 z}-\frac{1}{3}\end{array}\right)$,

which corresponds to the well-known traceless nematic tensor order parameter. Also, we can write the compact form

$q^{\mu \nu}=\left(n_{1 \mu} n_{1 v}-\frac{1}{3} \delta_{\mu \nu}\right)$

where $\mu, v=x, y, z$.

In order to account for the biaxiality, Sonnet, Virga, and Durand consider two additional unit vectors, $\vec{n}_{2}$ and $\vec{n}_{3}$, along the two minor axes of a nematogenic molecule, so that $\vec{n}_{2}$ is normal to $\vec{n}_{3}$, and both of them are normal to the director $\vec{n}_{1}$ along the major axis $\left(\vec{n}_{2} \cdot \vec{n}_{3}=0\right.$ and $\vec{n}_{1} \cdot \vec{n}_{2}=\vec{n}_{1} \cdot \vec{n}_{3}=0$ ). We then define a second traceless tensor,

$\boldsymbol{b}=\vec{n}_{2} \otimes \vec{n}_{2}-\vec{n}_{3} \otimes \vec{n}_{3}$,

which can be written as

$\mathbf{b}=\left(\begin{array}{lll}n_{2 x} n_{2 x}-n_{3 x} n_{3 x} & n_{2 x} n_{2 y}-n_{3 x} n_{3 y} & n_{2 x} n_{2 z}-n_{3 x} n_{3 z} \\ n_{2 y} n_{2 x}-n_{3 y} n_{3 x} & n_{2 y} n_{2 y}-n_{3 y} n_{3 y} & n_{2 y} n_{2 z}-n_{3 y} n_{3 z} \\ n_{2 z} n_{2 x}-n_{3 z} n_{3 x} & n_{2 z} n_{2 y}-n_{3 z} n_{3 y} & n_{2 z} n_{2 z}-n_{3 z} n_{3 z}\end{array}\right)$

In a more compact form, we write

$b^{\mu v}=\left(n_{2 \mu} n_{2 v}-n_{3 \mu} n_{3 v}\right)$, from which it is easy to see that

$\operatorname{Tr} \mathbf{b}=\sum_{\mu} b^{\mu \mu}=0$,

since $\vec{n}_{2}$ and $\vec{n}_{3}$ are unit vectors.

Consider a pair of nematogenic molecules, which are associated with the local tensor parameters $(\mathbf{q}, \mathbf{b})$ and $\left(\mathbf{q}^{\prime}, \mathbf{b}^{\prime}\right)$. We now write the orientational interaction energy between these molecules,

$V=-U_{0}\left\{\mathbf{q} \cdot \mathbf{q}^{\prime}+\gamma\left(\mathbf{q} \cdot \mathbf{b}^{\prime}+\mathbf{b} \cdot \mathbf{q}^{\prime}\right)+\lambda \mathbf{b} \cdot \mathbf{b}^{\prime}\right\}$,

where $U_{0}>0$ is a typical interaction energy parameter, and the dimensionless parameters $\gamma$ and $\lambda$ may be arbitrarily chosen. If $\gamma=\lambda=0$, we recover the interaction energy associated with the usual form of the Maier-Saupe model, which is known to lead to a uniaxial nematic phase. If either $\gamma \neq 0$ or $\lambda \neq 0$, the biaxial components contribute to the interaction energy, and we may anticipate the existence of a biaxial nematic phase. As it has been pointed out by Sonnet, Virga, and Durand [5], this expression of $V$ is the most general orientational form of the interaction energy between a pair of molecules if we restrict to linear terms of the products of the tensor order parameters and require invariance under the exchange of molecules. Also, this expression of $V$ corresponds to the general form of the pair interaction energy that has been proposed in the pioneering work of Straley [7].

According to the mean-field calculation of Sonnet, Virga, and Durand [5], there is a wide range of acceptable values of the parameters $\gamma$ and $\lambda$. For example, we can make the choice $\gamma=0$, which eliminates the couplings between uniaxial and biaxial terms. The interaction energy in this case reduces to the expression

$V=-U_{0}\left\{\mathbf{q} \cdot \mathbf{q}^{\prime}+\lambda \mathbf{b} \cdot \mathbf{b}^{\prime}\right\}$.

Besides the expected isotropic-uniaxial-biaxial sequence of phases, it has been shown that there is a tricritical point in a phase diagram in terms of $\lambda$ and the temperature $T$. In a more recent work, De Matteis and Virga [6] have shown that there are indeed two distinct tricritical points. In the present article, we show that calculations for the corresponding lattice statistical model, with the suppression of most orientational fluctuations, do lead to the same qualitative behavior.

An interesting choice of parameters, $\lambda=\gamma^{2}$, is related to the geometric-mean approximation, which is suggested by an analysis of London's dispersion forces, and which is the most investigated case in the literature (see, for example, the recent work by Luckhurst and collaborators [14]). The interaction energy in this case assumes the simple form

$V=-U_{0}(\mathbf{q}+\gamma \mathbf{b}) \cdot\left(\mathbf{q}^{\prime}+\gamma \mathbf{b}^{\prime}\right)$. 
Again, the elementary lattice model reproduces all the qualitative findings of more involved calculations.

\section{Statistical Formulation of the SVD Model}

Given the general form of the interaction energy between pairs of molecules, (8), we write a fully-connected lattice Hamiltonian,

$\mathcal{H}=-\frac{U_{0}}{2 N} \sum_{i, j=1}^{N} \sum_{\mu \nu}\left[q_{i}^{\mu \nu} q_{j}^{\mu \nu}+\gamma\left(q_{i}^{\mu \nu} b_{j}^{\mu \nu}+b_{i}^{\mu \nu} q_{j}^{\mu \nu}\right)+\lambda b_{i}^{\mu v} b_{j}^{\mu \nu}\right]$,

where $i, j=1,2, \ldots N$, and $\mu, v=x, y, z$. It is then

convenient to rewrite this Hamiltonian in the form

$\mathcal{H}=-\frac{U_{0}}{2 N} \sum_{\mu \nu}\left[\left(\sum_{i=1}^{N} q_{i}^{\mu \nu}\right)^{2}+2 \gamma\left(\sum_{i=1}^{N} q_{i}^{\mu \nu}\right)\left(\sum_{i=1}^{N} b_{i}^{\mu \nu}\right)+\lambda\left(\sum_{i=1}^{N} b_{i}^{\mu \nu}\right)^{2}\right]$

In the Zwanzig-type models, with local directors restricted to the Cartesian directions, as we explicitly indicate in
Appendix A, this expression is further simplified, since the local tensors do not have off-diagonal elements. In this case, we have

$\mathcal{H}=-\frac{U_{0}}{2 N} \sum_{\mu=1,2,3}\left[\left(\sum_{i=1}^{N} q_{i}^{\mu \mu}\right)^{2}+2 \gamma\left(\sum_{i=1}^{N} q_{i}^{\mu \mu}\right)\left(\sum_{i=1}^{N} b_{i}^{\mu \mu}\right)+\lambda\left(\sum_{i=1}^{N} b_{i}^{\mu \mu}\right)^{2}\right]$. by

The canonical partition function of this problem is given

$Z=\operatorname{Tr} \exp \left\{\frac{\beta U_{0}}{2 N} \sum_{\mu}\left[\left(\sum_{i=1}^{N} q_{i}^{\mu \mu}\right)^{2}+2 \gamma\left(\sum_{i=1}^{N} q_{i}^{\mu \mu}\right)\left(\sum_{i=1}^{N} b_{i}^{\mu \mu}\right)+\lambda\left(\sum_{i=1}^{N} b_{i}^{\mu \mu}\right)^{2}\right]\right\}$

where $\beta=1 / T$ is the inverse of temperature $T$, and the trace indicates a sum over the (microscopic) configurations

of the tensors $\mathbf{q}$ and $\mathbf{b}$. We now introduce a new parameter,

$\omega^{2}=\lambda-\gamma^{2}$,

and write

$Z=\operatorname{Tr} \exp \left\{\sum_{\mu} \frac{\beta U_{0}}{2 N}\left[\sum_{i=1}^{N}\left(q_{i}^{\mu \mu}+\gamma b_{i}^{\mu \mu}\right)\right]^{2}+\frac{\beta U_{0}}{2 N} \omega^{2}\left[\sum_{i=1}^{N} b_{i}^{\mu \mu}\right]^{2}\right\}$

If $\omega^{2}>0$, it is quite standard to linearize the quadratic terms by the introduction of two integral Gaussian identities. Otherwise, if $\omega^{2}<0$, we can resort to integral representations of two Dirac delta functions. Note the particular cases: (i) $\gamma=\lambda=0$, which corresponds to the well-known uniaxial model; (ii) $\omega=0$, in other words, $\lambda=\gamma^{2}$, which corresponds to the most widely used case (and to the geometrical mean approximation of Luckhurst and collaborators [3]); (iii) $\gamma=0$, which has been analyzed in detail by Sonnet and collaborators [5], and by De Matteis and Virga [6]. 
We now resort to a set of Gaussian integral identities of the form

$$
\int_{-\infty}^{+\infty} \frac{d x}{\sqrt{\pi}} \exp \left(-x^{2}+2 a x\right)=\exp \left(a^{2}\right)
$$

With a convenient choice of variables, and for $\omega^{2}>0$, it is easy to write

$$
\begin{aligned}
Z= & \prod_{\mu}\left(\int\left(\frac{\beta U_{0} N}{2 \pi}\right)^{1 / 2} d x_{\mu} \int\left(\frac{\beta U_{0} N}{2 \pi}\right)^{1 / 2} d y_{\mu}\right) \exp \left[-\frac{1}{2} \beta U_{0} N \sum_{\mu}\left(x_{\mu}^{2}+y_{\mu}^{2}\right)\right] \\
& \times\left\{\operatorname{Tr} \exp \left[\sum_{\mu} \beta U_{0} x_{\mu}\left(q^{\mu \mu}+\gamma b^{\mu \mu}\right)+\sum_{\mu} \beta U_{0} x_{\mu} \omega y_{\mu} b^{\mu \mu}\right]\right\}^{N},
\end{aligned}
$$

where the single-site trace has to be calculated with the microscopic configurations given in Appendix A. If we carry out the sum over these accessible microscopic configurations, it is straightforward to show that

$Z=\int\left[d x_{\mu} d y_{\mu}\right] \exp \left[-\frac{1}{2} \beta U_{0} N \sum_{\mu}\left(x_{\mu}^{2}+y_{\mu}^{2}\right)+N \ln 2-\frac{1}{2} \beta U_{0} N \sum_{\mu} x_{\mu}+N \ln \mathcal{Z}_{0}\right]$,

with

$$
\begin{aligned}
\mathcal{Z}_{0}= & \exp \left(\beta U_{0} x_{1}\right) \cosh \left[\beta U_{0} \gamma\left(x_{2}-x_{3}\right)+\beta U_{0} \omega\left(y_{2}-y_{3}\right)\right] \\
& +\exp \left(\beta U_{0} x_{2}\right) \cosh \left[\beta U_{0} \gamma\left(x_{1}-x_{3}\right)+\beta U_{0} \omega\left(y_{1}-y_{3}\right)\right] \\
& +\exp \left(\beta U_{0} x_{3}\right) \cosh \left[\beta U_{0} \gamma\left(x_{1}-x_{2}\right)+\beta U_{0} \omega\left(y_{1}-y_{2}\right)\right]
\end{aligned}
$$

Therefore, we write

$Z=\int\left[d x_{\mu} d y_{\mu}\right] \exp \left[-\beta U_{0} N f\right]$,

and minimize the density of free energy $f$ with respect to the variables $\left\{x_{\mu}\right\}$ and $\left\{y_{\mu}\right\}$.

From the saddle-point equations,

$\frac{\partial f}{\partial x_{\mu}}=0 ; \quad \frac{\partial f}{\partial y_{\mu}}=0$,

we show that

$\sum_{\mu} x_{\mu}=\sum_{\mu} y_{\mu}=0$,

which is the traceless property of the tensors $\boldsymbol{x}$ and $\boldsymbol{y}$. We thus have to analyze a system of just four equations and four unknowns, and it is not difficult to guess some acceptable solutions.

From the physical point of view, this problem is more appealing if we introduce the change of variables,

$x_{\mu}=Q_{\mu}+\gamma B_{\mu}$

and

$y_{\mu}=\omega B_{\mu}$.

In terms of these new variables, we have

$Z=\int\left[d Q_{\mu} d B_{\mu}\right] \exp \left[-\beta U_{0} N f\right]$

so that

$$
\begin{aligned}
f= & \frac{1}{2} \sum_{\mu}\left[Q_{\mu}^{2}+2 \gamma Q_{\mu} B_{\mu}+\lambda B_{\mu}^{2}\right] \\
& -\frac{1}{\beta U_{0}} \ln 2-\frac{1}{3} \sum_{\mu}\left(Q_{\mu}+\gamma B_{\mu}\right)-\frac{1}{\beta U_{0}} \ln \widetilde{\mathcal{Z}_{0}},
\end{aligned}
$$

with

$$
\begin{aligned}
\widetilde{Z}_{0}= & \exp \left[\beta U_{0}\left(Q_{1}+\gamma B_{1}\right)\right] \cosh \left\{\beta U_{0}\left[\gamma\left(Q_{2}-Q_{3}\right)+\lambda\left(B_{2}-B_{3}\right)\right]\right\} \\
& +\exp \left[\beta U_{0}\left(Q_{2}+\gamma B_{2}\right)\right] \cosh \left\{\beta U_{0}\left[\gamma\left(Q_{1}-Q_{3}\right)+\lambda\left(B_{1}-B_{3}\right)\right]\right\} \\
& +\exp \left[\beta U_{0}\left(Q_{3}+\gamma B_{3}\right)\right] \cosh \left\{\beta U_{0}\left[\gamma\left(Q_{1}-Q_{2}\right)+\lambda\left(B_{1}-B_{2}\right)\right]\right\}
\end{aligned}
$$


Now, it is relatively easy to obtain the minima of the function $f$ and to check the traceless property,

$\sum_{\mu} Q_{\mu}=\sum_{\mu} B_{\mu}=0$.

These expressions are particularly convenient to be used for writing an expansion of the free energy in the vicinity the isotropic phase and comparing with a general Landau-de Gennes phenomenological expansion.

\section{Analyses of Some Special Cases}

We now consider some special cases of our general expressions. As we have mentioned in the Introduction, we first show that the simplest choice of interaction parameters, corresponding to the simple MSZ model, leads to the weak first-order transition between an isotropic and a uniaxial nematic phase. We then analyze a special choice of parameters that leads to a phase diagram with a rich tricritical behavior. Finally, we consider a choice of parameters according to the usual geometric mean approximation, which leads to the well-known isotropic-uniaxial-biaxial sequence of phases, and to a Landau multicritical point.

\subsection{Uniaxial Nematic Transition}

The uniaxial nematic transition may be described by the simple pair interaction energy

$V_{u}=-U_{0} \mathbf{q} \cdot \mathbf{q}^{\prime}=-U_{0} \sum_{\mu \nu} q^{\mu \nu}\left(q^{\prime}\right)^{\mu \nu}$,

which can also be written as

$V_{u}=-U_{0}\left(\vec{n}_{1} \cdot \vec{n}_{1}^{\prime}\right)^{2}+U_{0}$

This expression corresponds to the uniaxial term of the general form of interaction energy proposed by Straley (see the general form of interaction in Appendix B).

This special case corresponds to the standard MaierSaupe model, which has been analyzed by numerous investigators. It leads to the usual weak first-order transition between the isotropic and the uniaxial nematic structure. In previous articles, we have shown that the restricted choice of the microscopic degrees of freedom, as described in Appendix A, leads to the definition of the Maier-SaupeZwanzig, or MSZ, lattice statistical model, with the typical thermodynamic behavior of any simple interactions of uniaxial type.

\subsection{Particular Case with $\gamma=0$ and $\lambda \neq 0$}

This particular case has been analyzed in detail by Sonnet, Virga, and Durand [5], who pointed out the existence of a novel tricritical behavior. Note that we can write

$$
\begin{aligned}
\mathbf{b} \cdot \mathbf{b}^{\prime}= & \sum_{\mu v}\left(n_{2 \mu} n_{2 v}-n_{3 \mu} n_{3 v}\right)\left(n_{2 \mu}^{\prime} n_{2 v}^{\prime}-n_{3 \mu}^{\prime} n_{3 v}^{\prime}\right) \\
= & \left(\vec{n}_{2} \cdot \vec{n}_{2}^{\prime}\right)^{2}-\left(\vec{n}_{2 \cdot} \cdot \vec{n}_{3}^{\prime}\right)^{2}-\left(\vec{n}_{3 \cdot \vec{n}_{2}^{\prime}}\right)^{2} \\
& +\left(\vec{n}_{3} \cdot{\overrightarrow{n^{\prime}}}_{3}\right)^{2},
\end{aligned}
$$

which corresponds to the last term in the biaxial contribution to Straley's interaction energy (see Appendix B). Therefore, if $\gamma=0$ and $\lambda \neq 0$, we have

$$
\begin{aligned}
V_{\lambda}=-U_{0}\{ & \left(\vec{n}_{1} \cdot{\overrightarrow{n^{\prime}}}_{1}\right)^{2}-1 \\
+ & +\lambda\left[\left(\vec{n}_{2} \cdot \vec{n}_{2}\right)^{2}-\left(\vec{n}_{2} \cdot \vec{n}_{3}^{\prime}\right)^{2}\right. \\
& \left.\left.-\left(\vec{n}_{3} \cdot \vec{n}_{2}^{\prime}\right)^{2}+\left(\vec{n}_{3} \cdot \vec{n}_{3}\right)^{2}\right]\right\} .
\end{aligned}
$$

In this case, it is convenient to write the lattice Hamiltonian

$$
\begin{aligned}
\mathcal{H}_{\lambda} & =-U_{0} \frac{1}{2 N} \sum_{i, j=1}^{N} \sum_{\mu \nu}\left[q_{i}^{\mu \nu} q_{j}^{\mu \nu}+\lambda b_{i}^{\mu \nu} b_{j}^{\mu \nu}\right] \\
& =-U_{0} \frac{1}{2 N} \sum_{\mu \nu}\left[\left(\sum_{i} q_{i}^{\mu \nu}\right)^{2}+\lambda\left(\sum_{i} b_{i}^{\mu \nu}\right)^{2}\right]
\end{aligned}
$$

which is associated with a partition function that may be easily simplified by the use of two Gaussian integral identities. Calculations are straightforward, and lead to a free energy functional and a set of equations of state that can be numerically analyzed in great detail.

In Fig. 1, we draw a phase diagram in terms of the parameter $\lambda$ and the temperature $T$ (in units of energy $U_{0}$ ). We indicate isotropic $(I)$, uniaxial $(U)$ and biaxial $(B)$ nematic regions. Broken lines indicate first-order transitions. The solid line represents the second-order transition between uniaxially and biaxially ordered nematic phases. In the inset of this figure we show a tricritical point (at the end of the second-order line) and a triple point (at the meeting of three first-order boundaries). This phase diagram qualitatively agrees with the predictions of the calculations by Sonnet and collaborators [5]. 


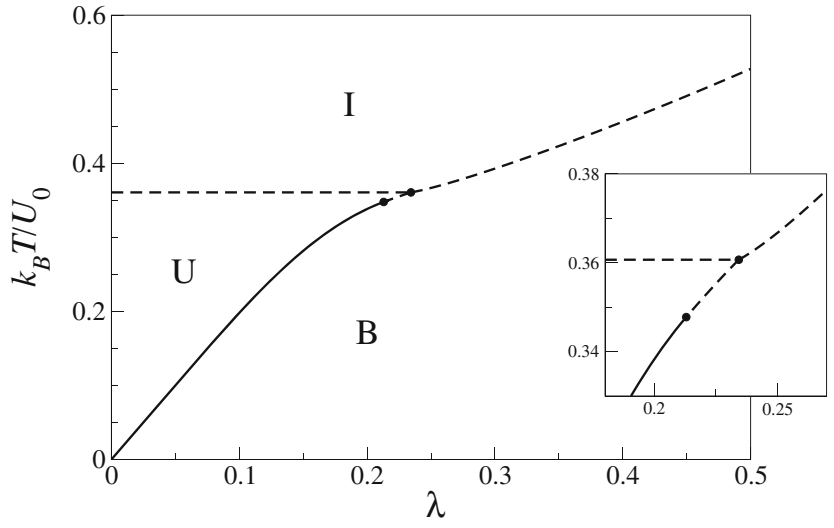

Fig. 1 Phase diagram in terms of temperature $T$ (in convenient units) and parameter $\lambda$, with $\gamma=0$. Dashed lines indicate first-order transitions (the solid line is a second-order transition). We also indicate nematic uniaxial $(U)$, nematic biaxial $(B)$ and isotropic $(I)$ regions. This diagram corresponds to the particular case analyzed by De Matteis and Virga

We now refer to Fig. 2, which is an amplification of Fig. 1 for $0.4 \lesssim \lambda \lesssim 0.8$. We clearly see a second tricritical point, in agreement with calculations of De Matteis and Virga [6]. Therefore, although it may be difficult to experimentally characterize the tricritical behavior, we show that the simple statistical lattice model reproduces the main findings of the recent (and much more involved) calculations for the phase diagram with this particular choice of energy parameters.

\subsection{Geometric Mean Approximation}

Most of the theoretical investigations of biaxial nematic structures use a pair interaction of the form

$$
\begin{aligned}
V_{l} & =-U_{0}[\mathbf{q}+\gamma \mathbf{b}] \cdot\left[\mathbf{q}^{\prime}+\gamma \mathbf{b}^{\prime}\right] \\
& =-U_{0}\left\{\mathbf{q} \cdot \mathbf{q}^{\prime}+\gamma\left(\mathbf{q} \cdot \mathbf{b}^{\prime}+\mathbf{b} \cdot \mathbf{q}^{\prime}\right)+\gamma^{2} \mathbf{b} \cdot \mathbf{b}^{\prime}\right\},
\end{aligned}
$$

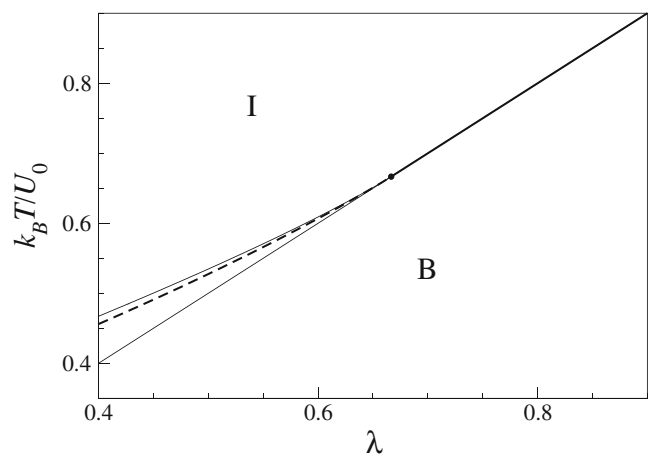

Fig. 2 Second tricritical point (for $\lambda>0.6$ ) in the $\lambda-T$ phase diagram with $\gamma=0$. The first-order boundary (dashed line) meets tangentially with the critical boundary (solid line) at a tricritical point. We also draw the stability lines of the isotropic and uniaxial nematic solutions

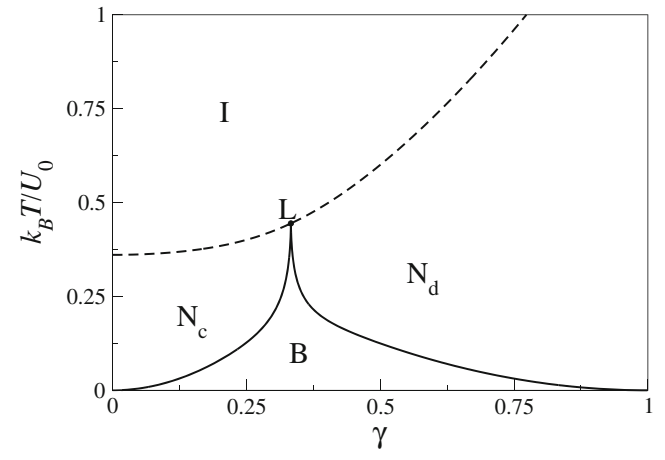

Fig. 3 Phase diagram in terms of temperature $T$ (in convenient units) and the parameter $\gamma$, with $\lambda=\gamma^{2}$, which corresponds to the usual geometric mean approximation. We indicate the isotropic $(I)$, biaxial $(B)$, and two distinct uniaxial nematic phases $\left(N_{c}\right.$ and $\left.N_{d}\right)$. The dashed line is a first-order transition. Solid lines are second-order transitions that meet at the Landau multicritcial point $(L)$

where $\gamma$ is a parameter of biaxiality. From the general expression of (8), we have

$\lambda=\gamma^{2} \quad \Longrightarrow \quad \omega^{2}=\lambda-\gamma^{2}=0$,

which corresponds to the geometric mean approximation of Luckhurst and collaborators (and to the simple MSZ6 model of our own previous article [4]).

According to the calculations of Section 2, it is convenient to define

$\mathbf{Q}=\mathbf{q}+\gamma \mathbf{b}$,

and write the usual quadratic energy for a Hamiltonian with pair interactions. In the language of the six-state model (as in Appendix A), it is easy to see that

$$
\begin{aligned}
\mathbf{Q}_{1} & =\left(\begin{array}{ccc}
2 / 3 & 0 & 0 \\
0 & -1 / 3 & 0 \\
0 & 0 & -1 / 3
\end{array}\right)+\gamma\left(\begin{array}{ccc}
0 & 0 & 0 \\
0 & 1 & 0 \\
0 & 0 & -1
\end{array}\right) \\
& =\left(\begin{array}{ccc}
2 / 3 & 0 & 0 \\
0 & -1 / 3+\gamma & 0 \\
0 & -1 / 3-\gamma
\end{array}\right),
\end{aligned}
$$

with the additional five tensors, $\mathbf{Q}_{2}$ to $\mathbf{Q}_{6}$, given by permutations of the diagonal elements.

In Fig. 3, we show a phase diagram that has been obtained by many authors (see, for example, the recent review of computer simulations by Berardi and coworkers [15]). 


\section{Conclusions}

We have used a general form of the interaction energy between pairs of molecules to introduce a class of fully connected elementary lattice models. If the microscopic nematic directors are restricted to point along the Cartesian axis, the statistical problem is amenable to standard calculations. We have used this approach to account for the main features of complex phase diagrams, in terms of temperature and a wide choice of model parameters. With the simplest choice of parameters, we reproduce the well-known weak first-order transition between the isotropic and the uniaxial nematic phases. With a choice of interaction parameters according to a geometric mean rule, which has been used by a large number of investigators, we reproduce the wellknown sequence of isotropic to uniaxial nematic and then to a biaxial nematic phase as a function of decreasing temperatures. Also, we locate a Landau multicritical point and can easily make contact with the standard Landau-de Gennes phenomenological expansions of the free energy, with the advantage of calculating the expansion coefficients in terms of the model parameters. For a special choice of parameters, we reproduce a phase diagram with a rich tricritical behavior, as it has been pointed out in the recent literature. We claim that these elementary lattice models can be easily used to explore new situations and to go beyond the simplest mean-field calculations.

\section{Appendix A}

In the six-state model, we have the following microscopic configurations of the local nematic directors:
(i) $\quad \vec{n}_{1}=( \pm 1,0,0) ; \quad \vec{n}_{2}=(0, \pm 1,0) ; \quad \vec{n}_{3}=(0,0, \pm 1)$
(ii) $\quad \vec{n}_{1}=( \pm 1,0,0) ; \quad \vec{n}_{2}=(0,0, \pm 1) ; \quad \vec{n}_{3}=(0, \pm 1,0)$
(iii) $\quad \vec{n}_{1}=(0, \pm 1,0) ; \quad \vec{n}_{2}=( \pm 1,0,0) ; \quad \vec{n}_{3}=(0,0, \pm 1)$
(iv) $\quad \vec{n}_{1}=(0, \pm 1,0) ; \quad \vec{n}_{2}=(0,0, \pm 1) ; \quad \vec{n}_{3}=( \pm 1,0,0)$
(v) $\vec{n}_{1}=(0,0, \pm 1) ; \quad \vec{n}_{2}=( \pm 1,0,0) ; \quad \vec{n}_{3}=(0, \pm 1,0)$
(vi) $\quad \vec{n}_{1}=(0,0, \pm 1) ; \quad \vec{n}_{2}=(0, \pm 1,0) ; \quad \vec{n}_{3}=( \pm 1,0,0)$

\section{Appendix B}

The most general form of the orientational interaction energy between two biaxial objects is given by the Straley formula,

$$
\begin{aligned}
V= & \alpha+\frac{1}{2} \beta\left[\left(\vec{n}_{1} \cdot \vec{n}_{1}\right)^{2}-1\right] \\
& +2 \gamma\left[\left(\vec{n}_{3} \cdot \vec{n}_{3}\right)^{2}-\left(\vec{n}_{2} \cdot \vec{n}^{\prime}\right)^{2}\right] \\
& +\frac{1}{2} \delta\left[\left(\vec{n}_{2} \cdot \vec{n}^{\prime}\right)^{2}-\left(\vec{n}_{2} \cdot \vec{n}_{3}\right)^{2}\right. \\
& \left.-\left(\vec{n}_{3} \cdot \vec{n}_{2}^{\prime}\right)^{2}+\left(\vec{n}_{3} \cdot \vec{n}_{3}\right)^{2}\right] .
\end{aligned}
$$

According to Straley [7], this expression is obtained under the following assumptions: (i) it is restricted to quadratic terms of sines and cosines of the relative directions, (ii) it is invariant under the inversion of the axes, and (iii) it is symmetric under the exchange of particles.

Also, according to the work of Straley, we can write

$$
V=\alpha+\beta F_{1}(\theta)+\gamma\left[F_{2}(\theta, \phi)+F_{3}(\theta, \psi)\right]+\delta F_{4}(\theta, \phi, \psi),
$$

in terms of four basis functions and the Euler angles $(\theta, \phi, \psi)$. If we restrict the calculations to molecules with the symmetry of a rectangular parallelepiped, the associated distribution functions are invariant under the transformations

$$
\begin{aligned}
\phi & \rightarrow \phi+\pi ; \\
\psi & \rightarrow \psi+\pi ; \\
\theta & \rightarrow \pi-\theta ; \quad \phi \rightarrow \pi-\phi ; \quad \psi \rightarrow-\psi,
\end{aligned}
$$

so that we have

$$
\begin{aligned}
& F_{1}=\frac{1}{2}\left(3 \cos ^{2} \theta-1\right) ; \quad F_{2}=\operatorname{sen}^{2} \theta \cos 2 \phi ; \quad F_{3}=\operatorname{sen}^{2} \theta \cos 2 \psi ; \\
& F_{4}=\frac{1}{2}\left(1+\cos ^{2} \theta\right) \cos 2 \phi \cos 2 \psi-\cos \theta \operatorname{sen} 2 \phi \operatorname{sen} 2 \psi
\end{aligned}
$$


The general form of interaction of Sonnet, Virga, and

Durand, which can be written as

$$
\begin{aligned}
V= & -U_{0}\left\{\left(\vec{n}_{1} \cdot \vec{n}_{1}{ }_{1}\right)^{2}-1\right. \\
& +\gamma\left[\left(\vec{n}_{1} \cdot \vec{n}_{2}\right)^{2}-\left(\vec{n}_{1} \cdot \vec{n}_{3}^{\prime}\right)^{2}+\left(\vec{n}_{2} \cdot \vec{n}_{1}^{\prime}\right)^{2}-\left(\vec{n}_{3} \cdot \vec{n}_{1}\right)^{2}\right] \\
& \left.+\lambda\left[\left(\vec{n}_{2} \cdot \vec{n}_{2}\right)^{2}-\left(\vec{n}_{2} \cdot \vec{n}_{3}^{\prime}\right)^{2}-\left(\vec{n}_{3} \cdot \vec{n}_{2}^{\prime}\right)^{2}+\left(\vec{n}_{3} \cdot \vec{n}_{3}\right)^{2}\right]\right\}
\end{aligned}
$$

has been shown to be equivalent to the Straley expression [5].

\section{References}

1. E. do Carmo, D.B. Liarte, S.R. Salinas, Phys. Rev. E 81, 062701 (2010)

2. E. do Carmo, A.P. Vieira, S.R. Salinas, Phys. Rev. E 83, 011701 (2011)

3. D.B. Liarte, S.R. Salinas, C.S.O. Yokoi, Phys. Rev. E, 84 (2011)

4. E.S. Nascimento, E.F. Henriques, A.P. Vieira, S.R. Salinas, Phys. Rev. E 92, 64-82 (2014). 2015.pore

5. A. Sonnet, E.G. Virga, G.E. Durand, Phys. Rev. E 67, 061701 (2003)
6. G. De Matteis, E.G. Virga, Phys. Rev. E 71, 061703 (2005)

7. J.P. Straley, Phys. Rev. A 10, 1881 (1974)

8. R. Zwanzig, J. Chem. Phys. 39, 1714 (1963)

9. M.J. de Oliveira, A.M. Figueiredo-Neto, Phys. Rev. A 34, 3481 (1986)

10. D.B. Liarte, S.R. Salinas, in Perspectives and Challenges in Statistical Physics and Complex Systems for the Next Decade, ed. by G. Viswanathan, M.G.E. Luz, E.P. Raposo (World Scientific, Singapore, 2014)

11. N. Boccara, R. Medjani, L. de Sèze, J. Phys. 38, 149 (1977)

12. G.R. Luckhurst, S. Romano, Mol. Phys. 40, 129 (1980)

13. G.R. Luckhurst, C. Zannoni, P.L. Nordio, U. Segre, Mol. Phys. 30, 1345 (1975)

14. G.R. Luckhurst, S. Naemura, T. Sluckin, K.S. Thomas, S.S. Turzi, Phys. Rev. E 85, 031705 (2012)

15. R. Berardi, L. Muccioli, S. Orlandi, M. Ricci, C. Zannoni, J. Phys. Condens. Matter 20, 463101 (2008) 


\title{
Elementary lattice models for the nematic transitions in liquid-crystalline systems
}

\author{
S. R. Salinas and E. S. Nascimento \\ Instituto de Física, Universidade de São Paulo, São Paulo, Brazil
}

\begin{abstract}
The Maier-Saupe theory of the nematic phase transitions, with the requirement that the molecular directors are restricted to the Cartesian axes, can be formulated as a fully-connected lattice problem, which is amenable to standard statistical-mechanics calculations. A simple three-state Maier-Saupe-Zwanzig (MSZ) model describes the weak first-order transition between uniaxial nematic and isotropic phases in liquid crystals, and provides a framework for several investigations. We describe calculations for a disordered MSZ model, which has been conceived to represent a binary mixture of rods and discs. Conflicting results in the literature about the stability of a long searched for biaxial phase are shown to be related to distinct treatments of disorder degrees of freedom. Effects of intrinsic biaxiality can be accounted for by analyzing a six-state MSZ model. We then used this model to investigate the phase diagrams of a mixture of uniaxial and intrinsically biaxial molecules, which is shown to display several nematic phases, reentrant regions, and a Landau multicritical point. We finally point out the connections of the elementary statistical models with a recent proposal of a mean-field two-tensor formalism.
\end{abstract}

\section{KEYWORDS}

Biaxial nematic; lattice statistical models; Maier-Sape theory

In some recent investigations of the stability of biaxial structures in liquid-crystalline systems, we used the standard Maier-Saupe mean-field theory of the nematic transition to introduce a class of elementary lattice statistical models [1-6]. According to an old proposal of Zwanzig to treat the Onsager model of rigid cylinders [7, 8], we restricted the microscopic nematic directors to point along the Cartesian axis. Due to this suppression of microscopic fluctuations, the statistical problem is considerably simplified. The simplest of these lattice models, with pair interactions of uniaxial nature, is a three-state model, which we call MSZ, and which is amenable to standard and detailed statistical-mechanics calculations. In the context of this MSZ model, we are able to describe the well-known first-order nematic phase transition, and can easily write the coefficients of series expansion of a free energy functional to make contact with the usual Landau - de Gennes phenomenological expansion.

It has been instructive to use the MSZ model to consider a binary mixture of interacting uniaxial elements, which mimics a mixture of discs and rods. This model system is an attempt to make contact with the diversity of micellar shapes in a lyotropic liquid crystal, and may

CONTACT S.R. Salinas ssalinas@if.usp.br E Instituto de Física, Universidade de São Paulo, São Paulo, 05508-090, Brazil. (C) 2017 Taylor \& Francis Group, LLC 
account for the existence of a biaxial phase, as it has been found in the early experiments of Yu and Saupe [9], and in a number of more recent experiments in São Paulo [10-12]. Models for a binary mixture of discs and rods had already been considered by several investigators, with conflicting results for the stability of a biaxial structure. According to the work of Palffy-Muhoray and collaborators [13], a biaxial structure cannot be thermodynamically stable. However, quite similar calculations by Henriques and Henriques [14, 15] did predict a temperature-concentration phase diagram with a thermodynamically stable biaxial phase, which is bordered by two distinct nematic structures, with critical lines ending at a Landau point. We have then decided to resort to the analysis of an elementary MSZ model for a binary mixture, which includes two distinct sets of degrees of freedom, orientational and shapedisordered degrees of freedom, and have shown that the stability of the biaxial structure depends on the ratio between the relaxation times associated with these distinct degrees of freedom $[1,4]$. According to our calculations, if orientational and shape degrees of freedom are both thermalized during the experimental time, there is no possibility of a thermodynamically stable biaxial phase, as it had been found by Palffy-Muhoray and collaborators. However, we predicted the existence of a biaxial structure if the shape-disorder degrees of freedom are quenched, fixed, as in a solid-state compound. Moreover, we have been able to use the MSZ formalism to show the possibility of occurrence of a biaxial structure for a small departure from complete thermalization.

We have as well introduced some modifications of the original MSZ model with the purpose of including some special forms of extra, intrinsically biaxial interactions, along the same lines that have been pursued in earlier theoretical investigations. This leads to definition of a six-state MSZ6 model, which may be shown to be equivalent to a lattice statistical model originally proposed by Boccara, Medjani, and de Sèze [16], to reproduce the wellknown sequence of isotropic, uniaxial, and biaxial nematic phases as a function of decreasing temperature [5].

The introduction of a six-state MSZ6 model opens the possibility of investigating the phase diagram of a binary mixture of intrinsically uniaxial and intrinsically biaxial molecular elements. We can draw a number of characteristic global phase diagrams, in terms of temperature $T$ and the concentration $c$ of intrinsically biaxial elements, for different values of a parameter $\Delta$ that gauges the degree of intrinsic biaxiality. If we consider shape degrees of freedom that are allowed to thermalize together with the orientational (quadrupolar) variables, it is easy to obtain rich phase structures, including uniaxial and biaxial nematic regions, reentrant boundaries, and many distinct multicritical points [5]. Also, we have used this formalism to write an expansion of the free energy in order to make contact with the Landau-de Gennes phenomenology.

More recently, we have investigated the connections of these statistical formulations with a proposal of a quite general two-tensor formalism, at the mean-field level, which is able to describe the classical isotropic-uniaxial-biaxial sequence of phases in the nematic systems as well as some novel features, as the onset of a tricritical point for a particular choice of interaction parameters $[17,18]$.

In Section 2, we introduce the MSZ statistical model and make some comments on the calculations for a binary system to mimic the phase diagram of a mixture of rods and discs. In Section 3, we comment on the connections of elementary statistical models with the meanfield, two-tensor, formalism of Sonnet, Virga, and Durand [17], which is known to be equivalent to an early proposal of Straley [19] for the most general form of the interaction between a pair of molecular units. 


\section{The elementary MSZ model}

According to the ideas of the Maier-Saupe theory for the nematic transition, we consider a lattice of $N$ sites and write the fully-connected Hamiltonian

$$
\mathcal{H}_{M S Z}=-\frac{A}{N} \sum_{1 \leq i<j \leq N} \sum_{\mu, v=x, y, z} S_{i}^{\mu \nu} S_{j}^{\mu \nu},
$$

where the first sum is over all pairs of lattice sites, and the parameter $A>0$ is suitably scaled by the number $N$ of sites in order to preserve the existence of the thermodynamic limit. The local "quadrupolar" degrees of freedom are given by

$$
S_{i}^{\mu \nu}=\frac{1}{2}\left(3 n_{i}^{\mu} n_{i}^{\nu}-\delta_{\mu \nu}\right)
$$

where $\vec{n}_{i}$ is the unit director associated with a molecule at site $i=1,2, \ldots, N$, and $\delta_{\mu \nu}$ is the Kronecker delta. In the MSZ model, we further assume that the local directors $\vec{n}_{i}$ are restricted to the Cartesian axes,

$$
\vec{n}_{i}=\left\{\begin{array}{l}
( \pm 1,0,0) \\
(0, \pm 1,0) \\
(0,0, \pm 1)
\end{array}\right.
$$

which leads to a three-state statistical model. It should be pointed out that this problem is amenable to quite simple and standard statistical-mechanics calculations. Moreover, this elementary MSZ model leads to essentially the same qualitative results as obtained from slightly more involved calculations for the original Maier-Saupe model with a continuous distribution of orientational degrees of freedom [20].

The consideration of a mixture of rods and discs is a simple and convenient starting point to investigate the existence of a biaxial phase, as in the experimental work of Yu and Saupe [9] for the phase diagram of a lyotropic system. In the presence of two types of degrees of freedom, one of them of orientational nature, as represented by the quadrupolar interactions, and the other one of shape-disorder nature, as represented by the presence of either rods or discs at the lattice sites, it is important to distinguish between quenched (fixed) and annealed (thermalized) types of disorder. The MSZ model turns out to provide a unifying and powerful tool to analyze this binary system in the quenched, annealed, and even intermediate regimes.

In the simplest three-state MSZ model for a mixture of rod-shaped and disc-shaped molecules, we include a disorder variable $\lambda_{i}$, for $1 \leq i \leq N$, and write the energy

$$
\mathcal{H}=-\frac{A}{N} \sum_{1 \leq i<j \leq N} \sum_{\mu, v=1,2,3} \lambda_{i} \lambda_{j} S_{i}^{\mu v} S_{j}^{\mu v},
$$

so that $\lambda_{i}=-1$ represents oblate-shaped molecules (discs), and $\lambda_{i}=+1$ represents prolateshaped molecules (rods), and $A>0$ is a coupling constant.

In the fixed (quenched) case, we assume that the disorder variables $\lambda_{i}$ are independent and identically distributed with probability

$$
P\left(\lambda_{i}\right)= \begin{cases}c, & \text { for } \lambda_{i}=+1 \\ 1-c, & \text { for } \lambda_{i}=-1\end{cases}
$$

This disordered model, as the MSZ model itself, is exactly solvable. Details of the calculations are given in our previous publications [1]. The free energy is self-averaging, so that it 


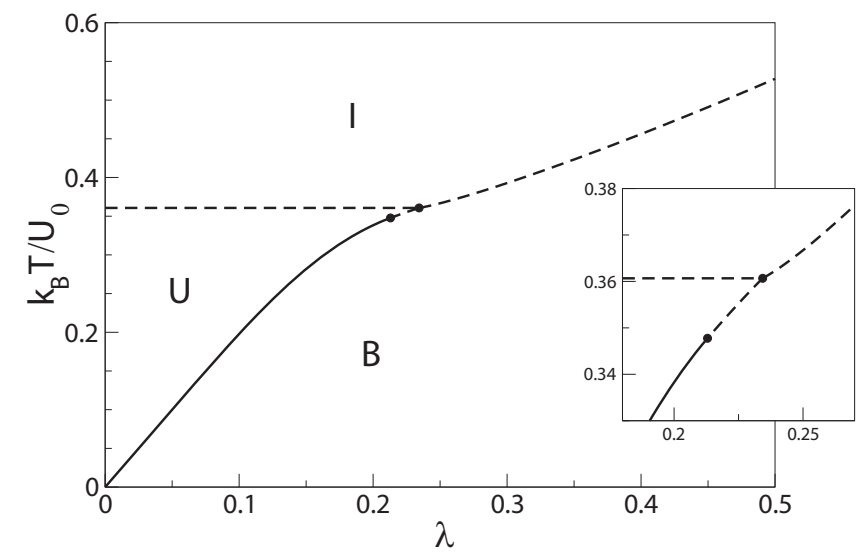

Figure 1. Phase diagram in terms of temperature $T$ (in convenient units) and parameter $\lambda$, with $\gamma=0$. Dashed lines indicate first-order transitions; the solid line is a second-order transition. We also indicate nematic uniaxial (U), nematic biaxial (B) and isotropic (I) regions.

is given by a typical configuration of the disorder variables, and we do not have to resort to the replica method. As it should be anticipated, the phase diagram, in terms of temperature $T$ and concentration $c$, displays an isotropic region, at high temperatures, and three nematic regions below a first-order boundary (see Figure 1 in the article by Carmo, Liarte and Salinas [1]). Two of these ordered structures are distinct nematic uniaxial phases (prolate and oblate phases, as in the experiment of Yu and Saupe). The intermediate structure is a biaxial nematic phase, which is separated from the uniaxial phases by two second-order lines that end at a Landau multicritical point. The biaxial structure is stable in this $T-c$ phase diagram.

In the annealed, thermalized case, the disorder variables are no longer fixed, but may change in order to minimize the free energy. Again, the free energy can be obtained by a standard calculation, but the resulting phase diagram does not display a thermodynamically stable biaxial phase $[1,4]$. There is just a first-order boundary of coexistence between two distinct nematic phases. The biaxial solution of this problem is still there, but it is associated with a larger value of the free energy, so it has to be thermodynamically discarded. These calculations indicate that the basic MSZ model works quite well, with either quenched (fixed) or annealed (mobile, thermalized) disorder variables, but the biaxial phase is stable for fixed (quenched) polymorphism only, as it had already been pointed in previous investigations for similar model systems [14]. Annealed polymorphism is certainly more adequate for liquid systems, but the annealed calculation is unable to predict the experimentally observable biaxial structure.

The general results for both quenched and annealed cases may be studied by means of an expansion of the free energy in terms of the invariants of second and third order, $I_{2}$ and $I_{3}$, of the tensor order parameter. From a Landau - de Gennes analysis, we know that it is necessary to have a positive coefficient $E$ of the term depending on the square of the cubic invariant, $E I_{3}{ }^{2}$, in order to stabilize a biaxial nematic phase in the immediate vicinity of the Landau multicritical point [21]. We have been able to find explicit expressions for all coefficients. In particular $E=1 / 15$ is positive in the quenched case, and negative, $E=-1 / 135$, in the annealed case, which further supports our conclusions, and makes room for the investigation of an intermediate case, between fully quenched and fully annealed distributions of shapes.

At this point, we considered a formalism that takes into account two different heat baths, one of them associated with the quicker orientational variables, of quadrupolar nature, which 
we call $\left\{\sigma_{i}\right\}$, and the other one associated with the slower shape-disordered degrees of freedom, which we call $\left\{\lambda_{i}\right\}$. We begin by assigning a conditional probability of occurrence of a configuration $\sigma$ of the orientational variables, for a given, and fixed, set of disorder variables,

$$
P(\sigma \mid \lambda)=\frac{1}{Z_{\sigma}} \exp [-\beta \mathcal{H}(\sigma, \lambda)]
$$

where $Z_{\sigma}$ is an unconstrained partition function,

$$
Z_{\sigma}=Z_{\sigma}(\lambda)=\sum_{\sigma} \exp [-\beta \mathcal{H}(\sigma, \lambda)]
$$

If the statistical problem is carried further out, it turns to be equivalent to the purely quenched case. Let us then assume that the disorder variables are not fixed, but that they are allowed to change with time in accordance with a set of Langevin equations,

$$
\Gamma \frac{\partial \lambda_{i}}{\partial t}=-z(t) \lambda_{i}-\frac{\partial \mathcal{H}}{\partial \lambda_{i}}+\eta_{i}(t)
$$

where $z(t)$ is a Lagrange multiplier associated with the chemical potential, $\left\langle\eta_{i}(t)\right\rangle=0$, and there is an Einstein relation,

$$
\left\langle\eta_{i}(t) \eta_{j}\left(t^{\prime}\right)\right\rangle=2 \Gamma T_{\lambda} \delta_{i j} \delta\left(t^{\prime}-t^{\prime}\right),
$$

where we have introduced the temperature $T_{\lambda}$ of a second heat bath associated with the disorder variables. We now replace $\partial \mathcal{H} / \partial \lambda_{i}$ by its average value, define an effective Hamiltonian, and carry out a conventional statistical mechanics calculation [1, 4]. It is natural to define a ratio $n=T / T_{\lambda}$ between the temperatures of the heat baths, which are associated with fast and slow degrees of freedom, and which should be considered as a ratio of two distinct relaxation times. From an exact expansion of a suitable thermodynamic potential about the Landau multicritical point, located at $\beta=4 / 3$, and $\mu=0$, where $\mu$ is a chemical potential, we have the coefficient $E=(2 / 27)\left[(9 / 10)-n \operatorname{sech}^{2}(2 n \mu / 3)\right]$. For $n=1$, we regain the previous annealed result. For $n<9 / 10$, which is a small departure from the fully annealed situation, the sign of $E$ indicates that the biaxial phase becomes stable.

In conclusion, using the basic MSZ model, we were able to investigate the effects of the type of disorder - quenched, annealed, or intermediate - on the stability of a biaxial nematic phase of a mixture of rod-like and disc-like molecules. The biaxial phase is stable in the quenched case, but thermodynamically unstable for thermalized disorder variables. In a situation of partial annealing, a small departure from the condition of total thermalization should be sufficient to recover thermodynamic stability.

\section{Connections with a mean-field two-tensor formalism}

In a relatively recent publication, Sonnet, Virga, and Durand [17] proposed a two-tensor formalism to investigate the nematic phase transitions. Besides the usual uniaxial tensor order parameter, given by

$$
q^{\mu \nu}=\left(n_{1 \mu} n_{1 v}-\frac{1}{3} \delta_{\mu \nu}\right)
$$

where the unit vector $\vec{n}_{1}$ is the usual director associated with a uniaxial molecule, they consider an extra, biaxial tensor order parameter, given by

$$
b^{\mu v}=\left(n_{2 \mu} n_{2 v}-n_{3 \mu} n_{3 v}\right),
$$


with two additional unit vectors, $\vec{n}_{2}$ and $\vec{n}_{3}$, along the two minor axes of a (brick) molecule, so that $\vec{n}_{2}$ is normal to $\vec{n}_{3}$, and both of them are normal to the director $\vec{n}_{1}$ along the major axis $\left(\vec{n}_{2} \cdot \vec{n}_{3}=0\right.$ and $\left.\vec{n}_{1} \cdot \vec{n}_{2}=\vec{n}_{1} \cdot \vec{n}_{3}=0\right)$.

In this treatment, the pair interaction between two molecules, associated with the local tensor parameters $(\mathbf{q}, \mathbf{b})$ and $\left(\mathbf{q}^{\prime}, \mathbf{b}^{\prime}\right)$, is given by

$$
V=-U_{0}\left\{\mathbf{q} \cdot \mathbf{q}^{\prime}+\gamma\left(\mathbf{q} \cdot \mathbf{b}^{\prime}+\mathbf{b} \cdot \mathbf{q}^{\prime}\right)+\lambda \mathbf{b} \cdot \mathbf{b}^{\prime}\right\},
$$

where $U_{0}>0$ is a typical energy parameter, and the dimensionless parameters $\gamma$ and $\lambda$ may be arbitrarily chosen. If $\gamma=\lambda=0$, we recover the interaction energy associated with the usual form of the Maier-Saupe model, which is known to lead to a uniaxial nematic phase. If either $\gamma \neq 0$ or $\lambda \neq 0$, the biaxial components contribute to the interaction energy, and we may anticipate the existence of a biaxial nematic phase. If we restrict the interactions to linear terms of the products of the tensor order parameters and require invariance under the exchange of molecules, this expression is the most general orientational form of the interaction energy between a pair of molecules Also, it does correspond to general form of the pair interaction energy that has been proposed in the pioneering work of Straley [19].

We now use this pair interaction energy to define a set of fully-connected statistical models, with the Zwanzig restrictions to the nematic directors. It is easy to see that we have to consider just six microscopic states,

$$
\begin{aligned}
& \text { (i) } \vec{n}_{1}=( \pm 1,0,0) ; \vec{n}_{2}=(0, \pm 1,0) ; \vec{n}_{3}=(0,0, \pm 1) \text {, } \\
& \text { (ii) } \vec{n}_{1}=( \pm 1,0,0) ; \vec{n}_{2}=(0,0, \pm 1) ; \vec{n}_{3}=(0, \pm 1,0) \text {, } \\
& \text { (vi) } \vec{n}_{1}=(0,0, \pm 1) ; \vec{n}_{2}=(0, \pm 1,0) ; \vec{n}_{3}=( \pm 1,0,0) \text {. }
\end{aligned}
$$

It is interesting to look at some special cases of physical interest:

(i) For $\gamma=\lambda=0$, we recover the simple three-state MSZ model (and the standard theory of Maier and Saupe for the uniaxial nematic transition).

(ii) The choice $\lambda=\gamma^{2}$, corresponds to the "geometric mean approximation", which has been extensively used in the literature. The phase diagram, in terms of temperature $T$ and the parameter $\gamma$, displays the well-known sequence of isotropic, uniaxial, and biaxial nematic phases, for a fixed value of $\gamma$, and decreasing values of temperature. We recover the well-known results of our MSZ6 model [6].

(iii) In the particular case $\gamma=0$ and $\lambda \neq 0$, there is no direct couplings between uniaxial and biaxial terms. We confirmed the detailed mean-field analysis of De Matteis and Virga [6]. In Figure 1, we show a plot of the phase diagram in terms of temperature $T$ and the parameter $\lambda$. The statistical calculations, with the restriction of the directors to point along the Cartesian directions, indicate the existence of two distinct tricritical points. Particular choices of this type may be relevant to explain some features of the more recently explored thermotropic liquid crystals.

\section{Conclusions}

In conclusion, we have used the mean-field Maier-Saupe theory of the nematic transition, with a restriction of the microscopic molecular directors to point along the Cartesian axis, to introduce a class of simple and exactly soluble lattice statistical models. We analyzed several cases of physical interest. We report detailed calculations for a disordered three-state model that has been conceived to account for the suggestions of a biaxial structure in the phase 
diagram of mixtures of rods and discs. We point out that conflicting results in the literature for the stability of this biaxial phase are related to distinct treatments of the shape-disorder degrees of freedom. Effects of intrinsic biaxiality can be accounted for by analyzing a six-state MSZ model. We mention an investigation of the phase diagram of a mixture of uniaxial and intrinsically biaxial molecules, which includes several nematic phases, reentrant regions, and a Landau multicritical point. We finally point out the connections of the elementary statistical models with a proposal of a mean-field two-tensor formalism to describe the nematic transitions.

\section{Acknowledgments}

We thank the support of the National Institute of Science and Technology of Complex Fluids (INCTFCx0 and of the Brazilian agencies FAPESP and CNPq.

\section{References}

[1] do Carmo, E., Liarte, D. B., \& Salinas, S. R. (2010). Phys. Rev. E 81, 062701.

[2] do Carmo, E., Vieira, A. P., \& Salinas, S. R. (2011). Phys. Rev. E 83, 011701.

[3] Liarte, D. B., Salinas, S. R., \& Yokoi, C. S. O. (2011). Phys. Rev. E 84.

[4] Henriques, E. F., \& Salinas, S. R. (2012). Eur. J. Phys. E 35, 14.

[5] Nascimento, E. S., Henriques, E. F., Vieira, A. P., \& Salinas, S. R. (2015). Phys. Rev. E 92, 062503.

[6] Nascimento, E. S., Vieira, A. P., \& Salinas, S. R. (2016). Braz. J. Phys. 46, 664.

[7] Zwanzig, R. (1963). J. Chem. Phys. 39, 1714.

[8] de Oliveira, M. J., \& Figueiredo-Neto, A. M. (1986). Phys. Rev. A 34, 3481.

[9] Yu, L. J., \& Saupe, A. (1980). Phys. Rev. Lett. 45, 1000.

[10] Figueiredo-Neto, A. M., \& Salinas, S. R. A. (2005). The Physics of Lyotropic Liquid Crystals: Phase Transitions and Structural Properties, Oxford University Press: New York.

[11] Amaral, L. Q. (2010). Liq. Cryst. 37, 627.

[12] Figueiredo Neto, A. M., \& Galerne, Y. (2015). Lyotropic Systems. In: Biaxial Nematic Liquid Crystals: Theory, Simulation and Experiment, Luckhurst, G. R. \& Sluckin, T. (Eds.) pp. 285-304. Wiley.

[13] Palffy-Muhoray, P., de Bruyn, J. R., \& Dunmur, D. A. (1985). J. Chem. Phys. 82, 5294.

[14] Henriques, E. F., \& Henriques, V. B. (1997). J. Chem. Phys. 107, 8036.

[15] Henriques, E. F., Passos, C. B., Henriques, V. B., \& Amaral, L. Q. (2008). Liq. Cryst. 35, 555.

[16] Boccara, N., Medjani, R., \& de Sèze, L. (1977). Journal de Physique 38, 149.

[17] Sonnet, A., Virga, E. G., \& Durand, G. E. (2003). Phys. Rev. E 67, 061701.

[18] De Matteis, G., \& Virga, E. G. (2005). Phys. Rev. E 71, 061703.

[19] Straley, J. P. (1974). Phys. Rev. A 10, 1881.

[20] Liarte, D. B., \& Salinas, S. R. (2014). In: Perspectives and Challenges in Statistical Physics and Complex Systems for the Next Decade, Viswanathan, G., Luz, M. G. E., \& Raposo, E. P. (Eds.) World Scientific: Singapore.

[21] Gramsbergen, E. F., Longa, L., \& de Jeu, W. H. (1986). Phys. Rep. 135, 195. 


\title{
Maier-Saupe model for a mixture of uniaxial and biaxial molecules
}

\author{
E. S. Nascimento, ${ }^{1}$ E. F. Henriques, ${ }^{2}$ A. P. Vieira, ${ }^{1, *}$ and S. R. Salinas ${ }^{1}$ \\ ${ }^{1}$ Instituto de Física, Universidade de São Paulo, Caixa Postal 66318, 05314-970 São Paulo, SP, Brazil \\ ${ }^{2}$ Instituto de Física e Matemática, Universidade Federal de Pelotas, Caixa Postal 354, 96010-900 Pelotas, RS, Brasil
}

(Received 1 July 2015; published 1 December 2015)

\begin{abstract}
We introduce shape variations in a liquid-crystalline system by considering an elementary Maier-Saupe lattice model for a mixture of uniaxial and biaxial molecules. Shape variables are treated in the annealed (thermalized) limit. We analyze the thermodynamic properties of this system in terms of temperature $T$, concentration $c$ of intrinsically biaxial molecules, and a parameter $\Delta$ associated with the degree of biaxiality of the molecules. At the mean-field level, we use standard techniques of statistical mechanics to draw global phase diagrams, which are shown to display a rich structure, including uniaxial and biaxial nematic phases, a reentrant ordered region, and many distinct multicritical points. Also, we use the formalism to write an expansion of the free energy in order to make contact with the Landau-de Gennes theory of nematic phase transitions.
\end{abstract}

DOI: 10.1103/PhysRevE.92.062503

PACS number(s): $61.30 . \mathrm{Cz}$

\section{INTRODUCTION}

The characterization of biaxial nematic phases in a number of thermotropic liquid-crystalline systems [1-3] stimulated a revival of interest in the investigation of theoretical models to describe biaxial structures [4]. About 40 years ago, Freiser [5] showed the existence of uniaxial and biaxial nematic phases in a generalization of the mean-field Maier-Saupe theory of the nematic transition with the addition of suitably asymmetric degrees of freedom. A nematic biaxial phase has also been shown to exist in a lattice model with steric interactions between platelets [6] and in a number of calculations for model systems with soft- and hard-core interactions [7-9]. The early experimental results, however, referred to a lyotropic liquidcrystalline mixture [10], which should be better represented by a model of uniaxial nematogenic elements $[11,12]$ and which motivated the use of an elementary version of the Maier-Saupe theory [13-15] to investigate a lattice statistical model for a binary mixture of cylinders and disks. We now propose an extension of this elementary model, along the lines of Freiser's generalization of the Maier-Saupe theory, in order to analyze the global phase diagram of a mixture of uniaxial and biaxial molecules.

In some analytical [16] and numerical [11] calculations, it has been pointed out that shape variations play an important role in the stability of biaxial nematic phases. In spite of the complexity of liquid-crystalline systems, whose complete description may require the introduction of more realistic, and necessarily involved, theoretical models, we believe that there is still room for the investigation of elementary statistical lattice models, with the addition of some ingredients that may be essential to describe the main features of the thermodynamic behavior. Along the lines of Freiser's early work, we then add extra degrees of freedom, of a biaxial nature, to an elementary lattice model, which leads to the definition of a six-state Maier-Saupe (MS6) model. This MS6 model is similar to an earlier proposal by Bocarra and collaborators [7] and may be regarded as a generalization of a previously used three-state Potts model to describe the uniaxial nematic

\footnotetext{
*apvieira@if.usp.br
}

transition [17]. Shape variations are taken into account by introducing a "biaxiality parameter," $\Delta$, and by considering a binary mixture of molecules with $\Delta=0$ (intrinsically uniaxial molecules) and $\Delta \neq 0$ (intrinsically biaxial molecules). This model system is sufficiently simple to be amenable to detailed statistical mechanics calculations for either quenched [18] or annealed mixtures of molecules. We then carry out calculations to obtain global phase diagrams and write an expansion of the free energy for comparison with the standard form of the phenomenological Landau-de Gennes theory of phase transitions.

This paper is organized as follows. In Sec. II, we define the MS6 model for a binary mixture of molecules and formulate the statistical problem. In Sec. III, we analyze the mean-field equations, draw a number of characteristic phase diagrams, and put the problem in the context of the Landau-de Gennes theory. Section IV is devoted to the final discussion and to some conclusions.

\section{THE SIX-STATE MAIER-SAUPE MODEL}

The standard formulations of the Maier-Saupe theory of nematic phase transitions [19] can be described in terms of the Hamiltonian

$$
\mathcal{H}=-\epsilon \sum_{(i, j)} \sum_{\alpha, \beta=1,2,3} \Omega_{i}^{\alpha \beta} \Omega_{j}^{\alpha \beta},
$$

where $\epsilon$ is a positive parameter, $(i, j)$ means that the sum is over pairs of molecules at sites $i$ and $j$, and $\boldsymbol{\Omega}_{i}$ is the symmetric traceless quadrupole tensor associated with a molecule at site $i$. In general, $\boldsymbol{\Omega}_{i}$ may be written in terms of the direction cosines or Euler angles which connect the laboratory and molecular frames. From the traceless condition, we write the eigenvalues, $\Lambda_{1}=-1+\Delta, \Lambda_{2}=-1-\Delta$, and $\Lambda_{3}=2$, of the tensor $\Omega$, where $\Delta$ is a parameter that gauges the degree of biaxiality [20]. The relation between $\Delta$ and molecular anisotropy is made explicit in the Appendix.

The problem is considerably simplified if we resort to a discretization of directions, which has been used to describe the isotropic-nematic transition [17]. We then assume that the principal molecular axes are restricted to the directions of the Cartesian coordinates of the laboratory. Therefore, the 
quadrupole tensor $\boldsymbol{\Omega}$ can assume only six states, represented by the matrices

$$
\begin{aligned}
& \left(\begin{array}{ccc}
-1+\Delta & 0 & 0 \\
0 & -1-\Delta & 0 \\
0 & 0 & 2
\end{array}\right), \quad\left(\begin{array}{ccc}
-1+\Delta & 0 & 0 \\
0 & 2 & 0 \\
0 & 0 & -1-\Delta
\end{array}\right), \\
& \left(\begin{array}{ccc}
-1-\Delta & 0 & 0 \\
0 & -1+\Delta & 0 \\
0 & 0 & 2
\end{array}\right), \quad\left(\begin{array}{ccc}
-1-\Delta & 0 & 0 \\
0 & 2 & 0 \\
0 & 0 & -1+\Delta
\end{array}\right) \text {, } \\
& \left(\begin{array}{ccc}
2 & 0 & 0 \\
0 & -1-\Delta & 0 \\
0 & 0 & -1+\Delta
\end{array}\right), \quad\left(\begin{array}{ccc}
2 & 0 & 0 \\
0 & -1+\Delta & 0 \\
0 & 0 & -1-\Delta
\end{array}\right) \text {, }
\end{aligned}
$$

which leads to the definition of the MS6 model. If the molecules are intrinsically uniaxial $(\Delta=0)$, we regain a threestate model, which has been used to describe the transition from the isotropic to the uniaxial nematic phase [17] and to investigate the existence of a biaxial nematic phase in a binary mixture of cylinders and disks [13-15].

The thermodynamic behavior of the MS6 model is determined from the canonical partition function

$$
Z=\sum_{\left\{\boldsymbol{\Omega}_{i}\right\}} \exp \left[\beta \epsilon \sum_{(i, j)} \sum_{\alpha=1,2,3} \Omega_{i}^{\alpha \alpha} \Omega_{j}^{\alpha \alpha}\right]
$$

where $\beta \epsilon=1 / T$, so that $T$ is the temperature in suitable units, and the first sum is over all microscopic configurations $\left\{\boldsymbol{\Omega}_{i}\right\}$ of this MS6 model. This problem is further simplified if we consider a fully connected model, with equal interactions between all pairs of sites. At this mean-field level, if $\Delta=0$, we anticipate just a first-order transition between an isotropic and a uniaxial nematic phase. If $\Delta \neq 0$, however, we can describe the transition to a stable biaxial nematic phase.

We now turn to a mixture of intrinsically uniaxial $(\Delta=0)$ and intrinsically biaxial $(\Delta \neq 0)$ molecules. In this mixture we have two sets of degrees of freedom: (i) orientational degrees of freedom, $\left\{\boldsymbol{\Omega}_{i}\right\}$, of a quadrupolar nature, and (ii) shape-disordered degrees of freedom, $\left\{\Delta_{i}\right\}$, with either $\Delta_{i}=0$ or $\Delta_{i}=\Delta \neq 0$, at all lattice sites. These two sets of degrees of freedom may be associated with quite different relaxation times, which leads to the distinction between annealed and quenched situations [18,24]. In the quenched case, the "shape-disordered" degrees of freedom never reach thermal equilibrium during the experimental times. Given a configuration $\left\{\Delta_{i}\right\}$, we calculate a partition function, $Z=$ $Z\left(\left\{\Delta_{i}\right\}\right)$, and a configuration-dependent free energy, $f\left(\left\{\Delta_{i}\right\}\right)$. The free energy of the system is the average of $f\left(\left\{\Delta_{i}\right\}\right)$ over the shape-disordered degrees of freedom, and the concentration of intrinsically biaxial molecules is not a true variable of equilibrium thermodynamics. In the annealed case, the two sets of degrees of freedom are supposed to thermalize during the experimental time, so that the concentration and chemical potential are thermodynamically conjugate variables. In the annealed case, given the concentration, both types of particles are free to move across the system in order to minimize the free energy. In this work, we consider annealed disorder only, which is more appropriate to a liquid-crystalline system.
In the annealed case, consider a binary mixture of $N_{1}$ intrinsically biaxial molecules $(\Delta \neq 0)$ and $N_{2}=N-N_{1}$ uniaxial molecules $(\Delta=0)$. Given the numbers of uniaxial and biaxial molecules, the canonical partition function is a sum over the orientational and disorder configurations,

$$
Z_{a}=\sum_{\left\{\boldsymbol{\Omega}_{i}\right\}\left\{\Delta_{i}\right\}} \sum^{\prime} \exp \left[\beta \epsilon \sum_{(i, j)} \sum_{\alpha=1,2,3} \Omega_{i}^{\alpha \alpha}\left(\Delta_{i}\right) \Omega_{j}^{\alpha \alpha}\left(\Delta_{j}\right)\right],
$$

where the prime in the second sum indicates the restriction

$$
\sum_{i=1}^{N} \Delta_{i}=N_{1} \Delta
$$

At this stage, in order to deal with the restricted sum in Eq. (4), it is convenient to introduce a chemical potential and change to a grand ensemble. First, we redefine the shape variable of molecule $i$ such that

$$
\Delta_{i}=n_{i} \Delta
$$

where

$$
n_{i}= \begin{cases}0 & \text { for a uniaxial object } \\ 1 & \text { for a biaxial object. }\end{cases}
$$

Then the grand partition function is given by

$$
\Xi_{a}=\sum_{\left\{\boldsymbol{\Omega}_{i}\right\}} \sum_{\left\{n_{i}\right\}} \exp \left[\beta \epsilon \sum_{(i, j)} \sum_{\alpha=1,2,3} \Omega_{i}^{\alpha \alpha}\left(n_{i}\right) \Omega_{j}^{\alpha \alpha}\left(n_{j}\right)+\beta \mu \sum_{i} n_{i}\right],
$$

where $\mu$ is the chemical potential, which controls the number of biaxial molecules. We remark that the sums over configurations in Eq. (8) are no longer restricted, which makes it possible to carry out the calculations in the mean-field limit, as detailed in the next section.

\section{MEAN-FIELD CALCULATIONS}

The mean-field version of the MS6 model is given by the Hamiltonian

$$
\begin{aligned}
\mathcal{H}_{\mathrm{MF}} & =-\frac{\epsilon}{2 N} \sum_{i, j=1}^{N} \sum_{\alpha=1}^{3} \Omega_{i}^{\alpha \alpha}\left(n_{i}\right) \Omega_{j}^{\alpha \alpha}\left(n_{j}\right) \\
& =-\frac{\epsilon}{2 N} \sum_{\alpha=1}^{3}\left[\sum_{i=1}^{N} \Omega_{i}^{\alpha \alpha}\left(n_{i}\right)\right]^{2}
\end{aligned}
$$

The grand partition function $\Xi_{a}$ in Eq. (8) can be factorized using three Gaussian identities,

$$
\begin{aligned}
& \exp \left[\frac{\beta \epsilon}{2 N}\left(\sum_{i=1}^{N} \Omega_{i}^{\alpha \alpha}\left(n_{i}\right)\right)^{2}\right] \\
& \propto \int_{-\infty}^{+\infty} d q_{\alpha} \exp \left[-\frac{\beta \epsilon N}{2} q_{\alpha}^{2}+\beta \epsilon q_{\alpha} \sum_{i=1}^{N} \Omega_{i}^{\alpha \alpha}\left(n_{i}\right)\right],
\end{aligned}
$$

with $\alpha \in\{1,2,3\}$. This factorization effectively decouples the problem of calculating the grand partition function, and the 
sums over $\left\{\boldsymbol{\Omega}_{i}\right\}$ and $\left\{n_{i}\right\}$ can be performed in a straightforward way, so that we can write

$$
\Xi_{a} \propto \int_{-\infty}^{+\infty} d q_{1} \int_{-\infty}^{+\infty} d q_{2} \int_{-\infty}^{+\infty} d q_{3} \exp (-N \beta \epsilon \psi),
$$

where $\psi$ is a functional of $\left\{q_{\alpha}\right\}$. In the thermodynamic limit, the integral can be calculated by standard saddle-point techniques. Thermodynamic equilibrium is then associated with the minimization of $\psi$ with respect to $\left\{q_{\alpha}\right\}$, from which we obtain self-consistent mean-field equations for these quantities. These equations show that $q_{1}+q_{2}+q_{3}=0$, which suggests the introduction of a symmetric traceless tensor,

$$
\mathbf{Q}=\left(\begin{array}{ccc}
q_{1} & 0 & 0 \\
0 & q_{2} & 0 \\
0 & 0 & q_{3}
\end{array}\right)
$$

as an appropriate thermal average of $\boldsymbol{\Omega}_{i}\left(n_{i}\right)$. Using the traceless condition, it is convenient to rewrite $\mathbf{Q}$ as

$$
\mathbf{Q}=\frac{1}{2}\left(\begin{array}{ccc}
-S-\eta & 0 & 0 \\
0 & -S+\eta & 0 \\
0 & 0 & 2 S
\end{array}\right)
$$

in terms of two scalar parameters, $S$ and $\eta$ [25]. The isotropic phase is given by $S=\eta=0$. The nematic uniaxial phase is given by $S \neq 0$ and $\eta=0$ (or $\eta= \pm 3 S$ ). In the biaxial phase, we have $S \neq 0$ and $\eta \neq 0$.

In the following paragraphs we write explicit expressions for the thermodynamic potentials of the uniform system and of the annealed binary mixture. From these expressions, it is easy to perform numerical calculations to draw a plethora of global phase diagrams in terms of the model parameters. In order to asymptotically check the numerical findings, and to make contact with established phenomenological results, we may also write an expansion of the thermodynamic potential in terms of the invariants of the tensor order parameter, $I_{n}=$ $\operatorname{Tr} \mathbf{Q}^{n}$, with $n=1,2, \ldots$ Due to the symmetry properties of $\mathbf{Q}$, all these invariants can be written as polynomials depending on two basic invariants, given by

$$
I_{2}=\operatorname{Tr} \mathbf{Q}^{2}=\frac{1}{2}\left(3 S^{2}+\eta^{2}\right)
$$

and

$$
I_{3}=\operatorname{Tr} \mathbf{Q}^{3}=\frac{3}{4} S\left(S^{2}-\eta^{2}\right) .
$$

Therefore, the usual form of the Landau-de Gennes expansion is written as

$$
\begin{aligned}
g= & g_{0}+\frac{A}{2} I_{2}+\frac{B}{3} I_{3}+\frac{C}{4} I_{2}^{2}+\frac{D}{5} I_{2} I_{3} \\
& +\frac{E}{6} I_{2}^{3}+\frac{E^{\prime}}{6} I_{3}^{2}+\cdots .
\end{aligned}
$$

According to this phenomenological expansion [27], there is a Landau multicritical point for $A=B=0$. In the vicinity of this Landau point, we can establish parametric expressions for the lines of phase transitions between the isotropic and the nematic phases.

We now consider the specific cases of uniform and annealed systems.

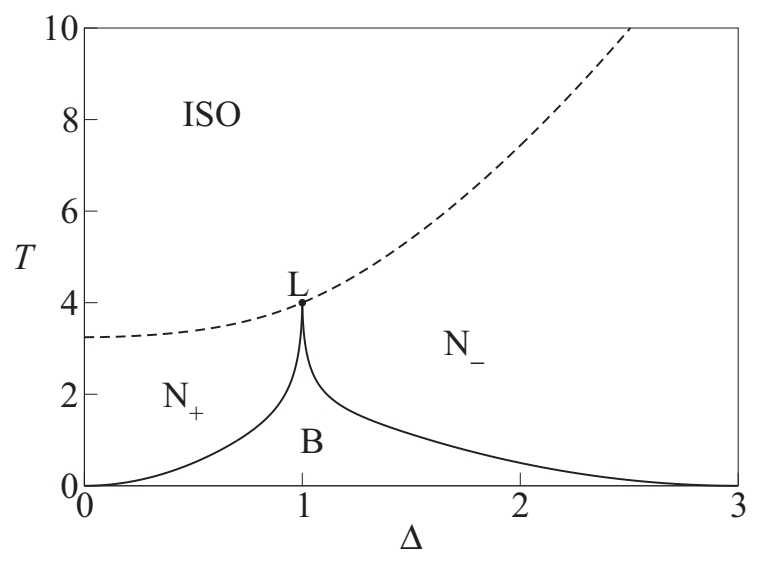

FIG. 1. Phase diagram, in terms of temperature $T$ and degree of biaxiality $\Delta$, for a system of intrinsically biaxial molecules. $\mathrm{N}_{+}$and $\mathrm{N}_{-}$are uniaxial nematic prolate and oblate phases, respectively. B, nematic biaxial phase; L, Landau multicritical point; ISO, isotropic region. Solid lines indicate continuous transitions; dashed lines, firstorder phase transitions.

\section{A. Uniform case}

Consider a system of intrinsically biaxial molecules $\left(\Delta_{i}=\right.$ $\Delta$ for all $i$ ). Assuming the discretization of orientations and setting $\mu=0$ in Eq. (8), since disorder plays no role, the functional $\psi$ is written as

$$
\begin{aligned}
\psi= & \frac{1}{2}\left(3 S^{2}+\eta\right)-T \ln 2-T \\
& \times \ln \left\{\exp \left[-\frac{3(S+\eta)}{2 T}\right] \cosh \left[\frac{(-3 S+\eta) \Delta}{2 T}\right]\right. \\
& +\exp \left[-\frac{3(-S+\eta)}{2 T}\right] \cosh \left[\frac{(3 S+\eta) \Delta}{2 T}\right] \\
& \left.+\exp \left(\frac{3 S}{T}\right) \cosh \left(\frac{\eta \Delta}{T}\right)\right\},
\end{aligned}
$$

where $T=(\beta \epsilon)^{-1}$. Minimizing $\psi$ with respect to $S$ and $\eta$ leads to the self-consistent mean-field equations, $S=F_{1}(S, \eta ; T, \Delta)$ and $\eta=F_{2}(S, \eta ; T, \Delta)$. The values of $S$ and $\eta$ at the absolute minimum of $\psi$ correspond to the thermodynamic equilibrium values for a fixed temperature $T$ and degree of biaxiality $\Delta$. The free energy $f=f(T, \Delta)$ of the system is obtained from $\psi$ by inserting the equilibrium values of $S$ and $\eta$.

Figure 1 shows the phase diagram in the $T-\Delta$ plane, which is obtained by solving the mean-field equations numerically. As should be anticipated from phenomenological arguments, this phase diagram shows two lines of continuous transitions (solid lines) from a biaxial nematic region to the $\mathrm{N}_{+}$(prolate) and $\mathrm{N}_{-}$(oblate) uniaxial nematic regions. These critical lines meet at a Landau multicritical point (L) on the first-order boundary (dashed lines) between the isotropic and the uniaxial nematic phases. It should be remarked that we regain an intrinsically uniaxial system for $\Delta=3$. The phase diagram for $\Delta>3$ is mapped onto the region $1<\Delta^{\prime}<3$ by the transformations $\Delta^{\prime}=(\Delta+3) /(\Delta-1)$ and $T^{\prime}=4 T /(\Delta-1)^{2}$. Note that a similar model for asymmetric ellipsoids leads to essentially the same type of phase diagram [7]. Also, a number 
of calculations for continuous orientational degrees of freedom lead to the same characteristic topology of this phase diagram (see, for example, the works by Luckhurst and collaborators [9] and Xheng and Palffy-Murhoray [28]).

From the expression of the free energy, we obtain the parameter-dependent coefficients of a Landau-de Gennes expansion about the Landau multicritical point:

$$
\begin{gathered}
A=1-\frac{3+\Delta^{2}}{T}, \quad B=\frac{9}{2}\left(\frac{\Delta^{2}-1}{T^{2}}\right), \\
C=\frac{1}{T^{3}}\left(\frac{9}{4}+\frac{3}{2} \Delta^{2}+\frac{1}{4} \Delta^{4}\right) \\
D=-\frac{45}{16 T^{4}}\left(\Delta^{4}+2 \Delta^{2}-3\right) \\
E=-\frac{1}{480 T^{5}}\left(41 \Delta^{6}+315 \Delta^{4}+1215 \Delta^{2}+1053\right), \\
E^{\prime}=\frac{1}{40 T^{5}}\left(\Delta^{6}+225 \Delta^{4}-405 \Delta^{2}+243\right) .
\end{gathered}
$$

Therefore, the Landau point $(\mathrm{L})$ is located at $T_{L}=4$ and $\Delta_{L}=1$. In the vicinity of the Landau point, limiting ourselves to first-order terms in $(T-4)$ and $(\Delta-1)$, we have $A=(1 / 4)(T-4)-(1 / 2)(\Delta-1), B=(9 / 16)(\Delta-1), C=$ $1 / 16, D=0, E=-41 / 7680$, and $E^{\prime}=1 / 640$. The sign of $E^{\prime}$ indicates the stability of the biaxial nematic phase near the Landau point $[19,27]$. In this mean-field scenario, at fixed $\Delta \neq 1$, as the temperature decreases from a sufficiently high value, the system goes from an isotropic phase to a uniaxial nematic phase and then to a biaxial nematic phase, according to the prediction in the early work by Freiser [5]. It should be remarked that the phenomenological Landau parameters are written in terms of the parameters of the underlying molecular model, which makes it easier to investigate a large range of values.

\section{B. Annealed disorder}

In the annealed case, we calculate $\psi$, given by Eq. (11), in terms of the proper thermodynamic field variables, temperature $T$, and chemical potential $\mu=\epsilon T \ln z$, where $z$ is the fugacity. For the fully connected MS6 model of a binary mixture of uniaxial and biaxial molecules, the functional $\psi$ is given by

$$
\psi=\frac{1}{2}\left(3 S^{2}+\eta^{2}\right)-T \ln 2-T \ln \sigma
$$

where

$$
\begin{aligned}
\sigma= & e^{-\frac{3(S+\eta)}{2 T}}\left\{1+z \cosh \left[\frac{(3 S-\eta) \Delta}{2 T}\right]\right\} \\
& +e^{\frac{-3(S-\eta)}{2 T}}\left\{1+z \cosh \left[\frac{(3 S+\eta) \Delta}{2 T}\right]\right\} \\
& +e^{\frac{3 S S}{T}}\left\{1+z \cosh \left(\frac{\eta \Delta}{T}\right)\right\} .
\end{aligned}
$$

Again, the thermodynamic stable values of $S$ and $\eta$ are chosen to minimize the function $\psi$ for fixed values of temperature $T$, degree of biaxiality $\Delta$, and chemical potential $\mu$.

Inserting the equilibrium values of $S$ and $\eta$ into the expression of $\psi$, we obtain the grand potential as a function of
$T, \mu$, and $\Delta$. If we wish to work with a fixed concentration $c$ of the intrinsically biaxial molecules, the free energy $\phi(c, T ; \Delta)$ comes from the definition

$$
\phi(c, T ; \Delta)=\psi+c \ln z,
$$

where the fugacity is eliminated by the expression

$$
c=-z \frac{\partial \psi}{\partial z}
$$

and we should insert the equilibrium values of $S$ and $\eta$ (with the proviso of a Maxwell construction whenever it is necessary).

The grand potential can be used to write a Landau-de Gennes expansion with coefficients

$$
\begin{gathered}
A=1-\frac{3+z\left(3+\Delta^{2}\right)}{T(1+z)}, \quad B=\frac{9}{2} \frac{z\left(\Delta^{2}-1\right)-1}{T^{2}(1+z)} \\
C=\frac{9+z\left[18+9 z+6(1+z) \Delta^{2}-(1-z) \Delta^{4}\right]}{4 T^{3}(1+z)^{2}} \\
D=\frac{15}{16} \frac{9+z\left[18-6 \Delta^{2}+\Delta^{4}-3 z\left(-3+2 \Delta^{2}+\Delta^{4}\right)\right]}{T^{4}(1+z)^{2}} \\
E=\frac{1053-z\left(c_{0}+z c_{1}+z^{2} c_{2}\right)}{480 T^{5}(1+z)^{3}}
\end{gathered}
$$

where

$$
\begin{gathered}
c_{0}=-3159-1215 \Delta^{2}+225 \Delta^{4}-11 \Delta^{6}, \\
c_{1}=-3159-2430 \Delta^{2}-90 \Delta^{4}+68 \Delta^{6}
\end{gathered}
$$

and

$$
\begin{gathered}
c_{2}=-1053-1215 \Delta^{2}-315 \Delta^{4}-41 \Delta^{6}, \\
E^{\prime}=\frac{243+z\left(d_{0}+z d_{1}\right)}{40 T^{5}(1+z)^{2}},
\end{gathered}
$$

where

$$
d_{0}=486-405 \Delta^{2}-45 \Delta^{4}+\Delta^{6}
$$

and

$$
d_{1}=243-405 \Delta^{2}+225 \Delta^{4}+\Delta^{6} .
$$

From the usual condition $A=B=0$, we locate a Landau multicritical point,

$$
\mu_{L}=-T_{L} \ln \left(\Delta^{2}-1\right), \quad T_{L}=4,
$$

which requires $\Delta^{2}>1$ and gives an indication of the existence of qualitatively different phase diagrams (as there is no Landau point for $\Delta^{2}<1$ ).

It is easy to show that this annealed version of the binary mixture exhibits phase diagrams with many distinct topologies. This can be anticipated from an analysis of the energy levels associated with the interaction between molecules, as shown in Fig. 2. In fact, these energy levels are associated with different degrees of degeneracy $\omega$, and some levels cross each other as the biaxiality of the molecules is changed. These degeneracies account for entropic contributions to the free energy, which do affect the equilibrium phase behavior of 


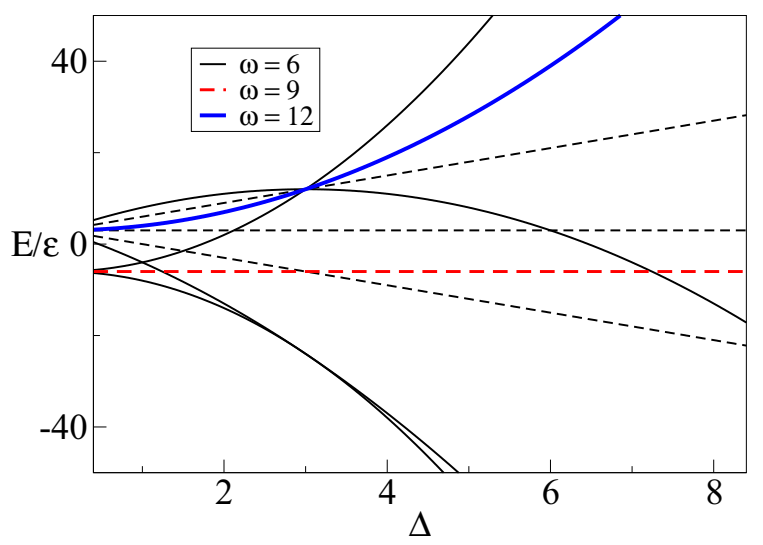

FIG. 2. (Color online) Energy levels as a function of the degree of biaxiality $\Delta$. Level crossings account for variations in the entropic contribution to the free energy. Solid lines correspond to energy levels associated with interactions between two biaxial molecules, while dashed lines indicate energy levels arising from interactions involving at least one uniaxial molecule. Line thickness is proportional to the degeneracy $\omega$ of the corresponding level.

the system. Therefore, we anticipate qualitative changes in the phase diagrams as $\Delta$ assumes values close to the location of the energy level crossings.

Contrary to what is seen in the uniform limit, in the presence of unixial molecules the phase diagrams shown below do not exhibit symmetry between $1<\Delta \leqslant 3$ and $\Delta>3$. This is due to the fact that the energy spectrum of the interactions involving uniaxial molecules (shown as dashed lines in Fig. 2) is not invariant under the transformation $\Delta \rightarrow(\Delta+3) /(\Delta-1), E \rightarrow 4 E /(\Delta-1)^{2}$, contrary to what is observed for the energy spectrum associated with the interactions between a pair of biaxial molecules. In other words, for our choice of interactions the presence of a rod-like solute distinguishes between the biaxial rod-like and the biaxial disk-like components.

We now discuss the various topologies exhibited by the phase diagrams as the biaxiality parameter is changed. Figures 3 and 4 show $T-\mu$ and $T-c$ phase diagrams for a fixed degree of biaxiality, $\Delta=1$, where $\mu$ is the chemical potential and $c$ is the concentration of intrinsically biaxial molecules. From the thermodynamic point of view, the firstorder boundaries (dashed lines) in the $T$ - $\mu$ plane are mapped into coexistence regions [shaded (gray) regions] in the $T-c$ plane. For low temperatures and intermediate concentrations, there is a coexistence region between the uniaxial prolate $\left(\mathrm{N}_{+}\right)$ and the biaxial (B) phases. However, at higher concentrations and intermediate temperatures, there is a second-order phase transition (solid line) between the $\mathrm{N}_{+}$and the biaxial phase. In fact, the phase diagram exhibits a tricritical point (TC) along the boundary between the $\mathrm{N}_{+}$and the biaxial phase. At higher temperatures, there is a first-order phase transition between $\mathrm{N}_{+}$and the isotropic (ISO) phase, with a very thin coexistence region, as shown in the inset. Note that an incipient Landau point appears at $c=1$, which corresponds to an infinite chemical potential, in agreement with the Landau-de Gennes expansion. Phase diagrams with a similar topology (but with no Landau point) can be drawn for $0<\Delta<1$.

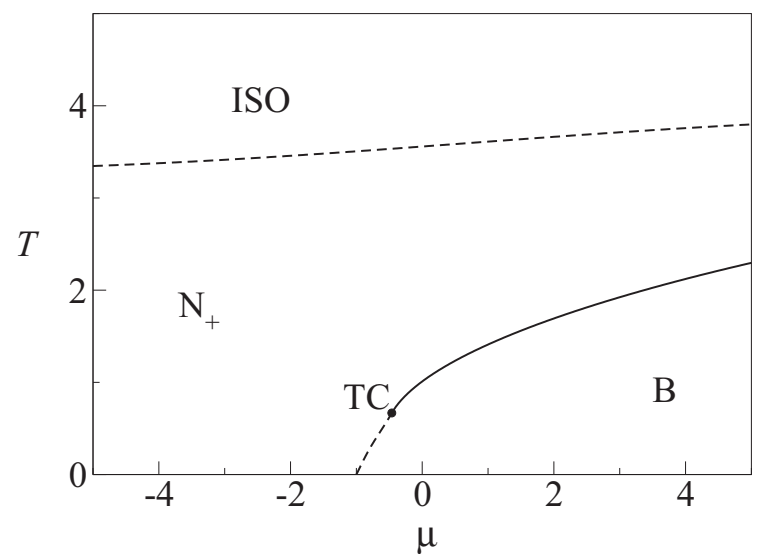

FIG. 3. Phase diagram for a fixed degree of biaxiality $\Delta=1$, where $T$ is the temperature and $\mu$ is the chemical potential. There is a tricritical point (TC) along the boundary separating biaxial (B) and uniaxial $\left(\mathrm{N}_{+}\right)$prolate nematic phases. There is no direct phase transition between the isotropic (ISO) and the biaxial phases (for finite values of the chemical potential).

Figure 5 shows the phase diagram for a fixed degree of biaxiality $\Delta=1.4$. Similarly to Fig. 4 , the $\mathrm{N}_{+}$and biaxial (B) phases coexist for intermediate concentrations and low temperatures. However, the system also displays a uniaxial oblate $\left(\mathrm{N}_{-}\right)$nematic phase, which appears at higher concentrations and intermediate temperatures. The biaxial phase appears between the two uniaxial phases, at intermediate temperatures. All three ordered phases become identical to the isotropic (ISO) phase at a Landau multicritical point (L). Also, note that the biaxial phase presents discrete reentrant behavior

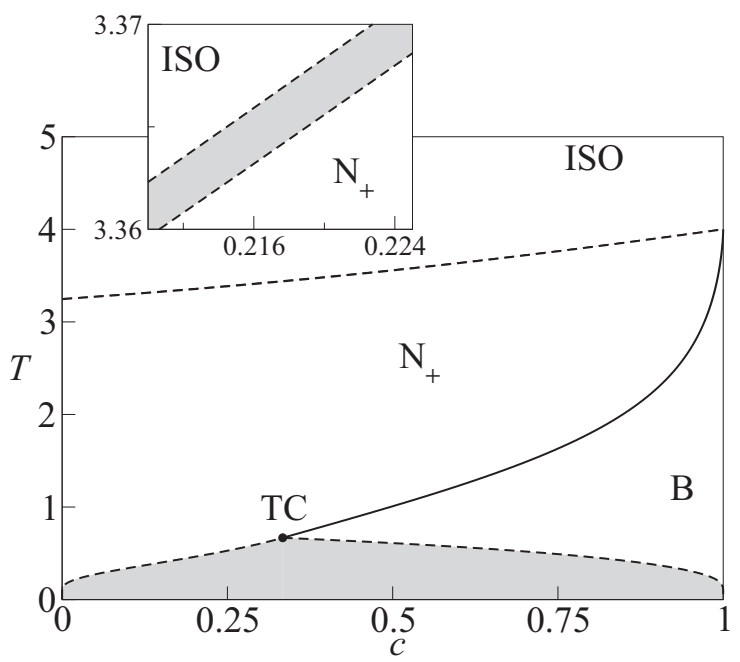

FIG. 4. Phase diagram in terms of the temperature $(T)$ versus the concentration $(c)$ of biaxial molecules, at a fixed degree of biaxiality, $\Delta=1$. There is an incipient Landau multicritical point at $c=1$, where the two ordered phases and the isotropic phase become identical. Uniaxial $\left(\mathrm{N}_{+}\right)$and biaxial (B) phases coexist at low temperatures and intermediate concentrations [shaded (gray) region]. The system exhibits a tricritical point (TC) along the boundary of the B phase. Inset: Zoom-in on the coexistence [shaded (gray)] region between the uniaxial nematic and the isotropic phases. 


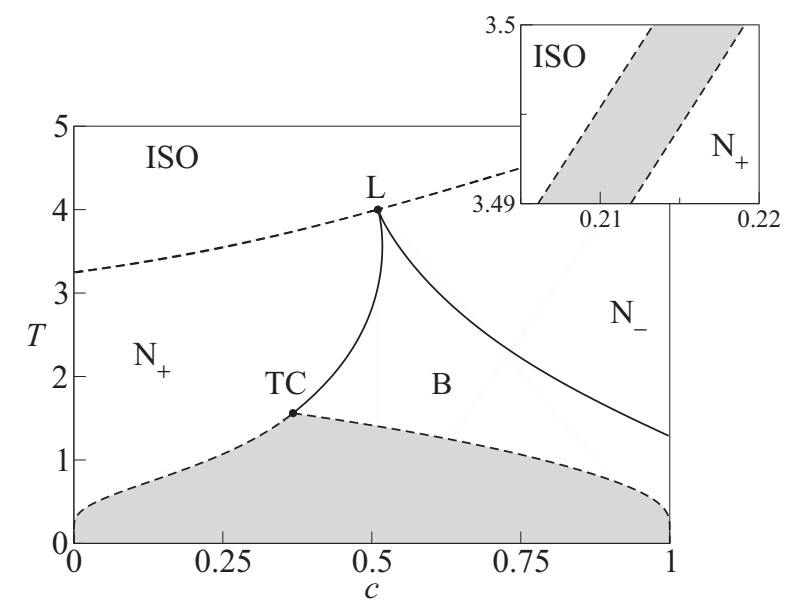

FIG. 5. Phase diagram, in terms of temperature $T$ and concentration $c$ of biaxial (B) molecules, for degree of biaxiality $\Delta=1.4$. There is a Landau point $(\mathrm{L})$. Note that the B phase displays reentrant behavior near $\mathrm{L}$.

close to the Landau multicritical point. Note that the changes in the topology of the phase diagrams shown in Figs. 4 and 5 are in agreement with the dependence of the energy levels on the degree of biaxiality $\Delta$ (see Fig. 2).

The phase behavior of the system changes significantly for a degree of biaxiality around $\Delta=1.5$, which is close to another crossing of energy levels. For example, in Fig. 6 we show the phase diagram for degree of biaxiality $\Delta=1.54$. The lowtemperature biaxial phase $\mathrm{B}_{-}$is represented by a tensor order parameter $\mathbf{Q}$, whose largest eigenvalue (in absolute value) is negative. However, an additional biaxial phase (B) appears near the Landau point. The two biaxial phases are stable in disconnected regions of the phase diagram. There is then a coexistence region between the uniaxial nematic phases $\mathrm{N}_{+}$ and $\mathrm{N}_{-}$. This region is limited by two critical end points (CE)

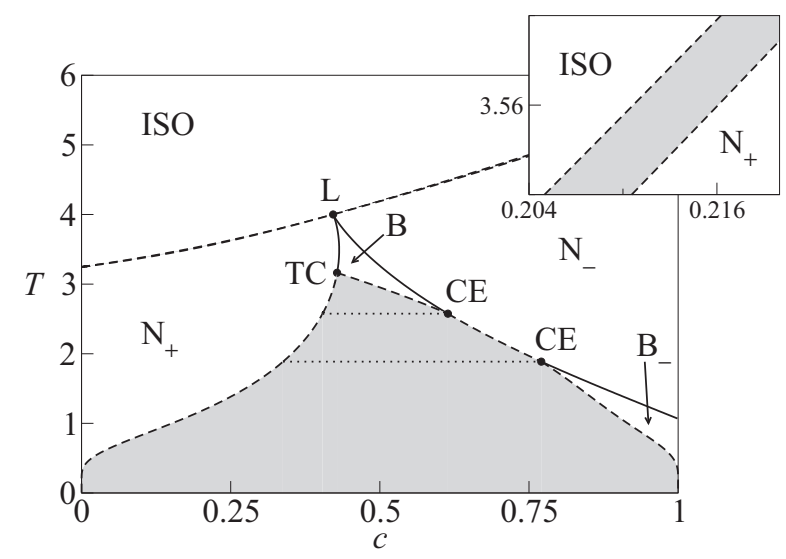

FIG. 6. Phase diagram in the $c-T$ plane for degree of biaxiality $\Delta=1.54$, where $T$ is the temperature and $c$ is the concentration of biaxial objects. Two nematic biaxial phases are stable in disconnected regions of the phase diagram. There is a coexistence region between the nematic uniaxial phases, ending at two critical end points (CE). Dotted horizontal lines represent special tie lines associated with the CEs.

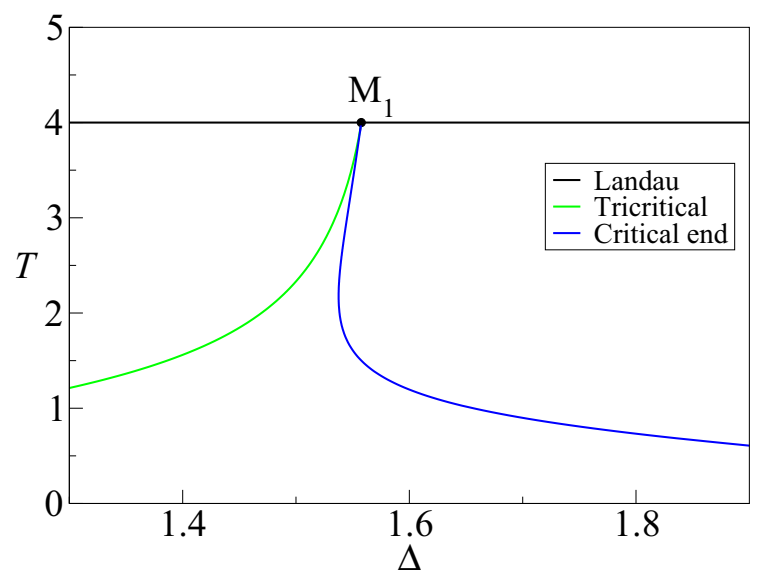

FIG. 7. (Color online) Lines of multicritical points in the $\Delta-T$ plane. There is a higher-order multicritical point $\mathrm{M}_{1}$, at which the lines of Landau points (horizontal black line), tricritical points [left-hand (green) line], and critical end points [right-hand (blue) line] meet. There is then no stable biaxial phase for $\Delta \geqslant \Delta_{\mathrm{M}_{1}}$ in the vicinity of the Landau point.

associated with the biaxial phases. Also, the system exhibits a tricritical point (TC) related to the coexistence region of $\mathrm{N}_{+}$and biaxial structures. As in the case $\Delta=1.4$, shown in Fig. 5, the biaxial phase is reentrant in the vicinity of the Landau point.

The topological changes in the phase diagram can be represented by the projections of the lines of different multicritical points on the $T-\Delta$ plane, as indicated in Fig. 7. The temperature of the Landau point (horizontal black line) is a constant function of the degree of biaxiality $\Delta$, which is in agreement with a Landau expansion. The temperature of the tricritical point [left-hand (green) line] increases monotonically with $\Delta$, as suggested by Figs. 4-6. However, the line of critical end points [right-hand (blue) line] presents reentrant behavior in the vicinity of $\Delta=1.55$, giving rise to the two critical end points, as shown in Fig. 6. All multicritical lines meet at a higher-order multicritical point $\mathrm{M}_{1}$. Note that the tricritical and high-temperature critical end points are associated with the biaxial phase near the Landau point. As a result, there is no stable biaxial phase in the vicinity of the Landau point for $\Delta \geqslant \Delta_{M_{1}} \simeq 1.5576$, although the low-temperature biaxial phase survives in a small region at high concentrations and low temperatures. This is illustrated in Fig. 8, for $\Delta=1.57$. There is no stable biaxial nematic phase close to the Landau point (L), which marks the meeting of various coexistence lines separating the isotropic phase and the two nematic uniaxial phases. Along those lines, there is a coexistence of phases with different values of nematic order parameter $S$, but the size of the coexistence region tends to vanish as we approach the Landau point.

As the degree of biaxiality is further increased, the system exhibits other distinct phase diagrams, as shown in Figs. 9 and 10. According to Fig. 2, multiple level crossings occur at $\Delta=3$, which is a strong suggestion of changes in the phase behavior of the system.

Figure 9 shows the phase diagram for $\Delta=2.9$. A triple point appears, associated with the coexistence of two uniaxial 


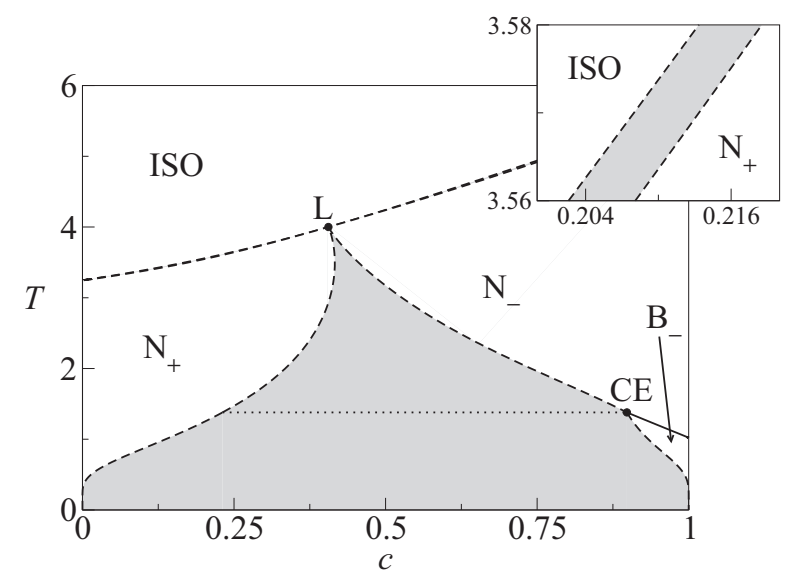

FIG. 8. Phase diagram in terms of temperature $(T)$ versus concentration $(c)$ of biaxial molecules for a fixed degree of biaxiality, $\Delta=1.57$. The biaxial phase $\mathrm{B}_{-}$is located at low temperatures and high concentrations. There is no stable B phase near the Landau point (L). The nematic uniaxial phases coexist in a region ending at a critical end point (CE). The dotted horizontal line represents a special tie line associated with the CE.

nematic oblate phases and a uniaxial prolate phase. Also, the coexistence region of uniaxial nematic oblate phases ends at a simple critical point (C). Although it is not shown in Fig. 9, a stable biaxial nematic phase is still present, at low temperatures and high concentrations, as well as the critical end point associated with the biaxial phase.

As $\Delta$ is further increased, the simple critical point moves upward in the phase diagram, approaching the lower border of the coexistence region between the uniaxial $\mathrm{N}_{-}$and the isotropic (ISO) phases. Then the simple critical point is replaced by a second triple point. For example, Fig. 10 represents a phase diagram for $\Delta=3$. Due to the second triple point, there appears an isolated second uniaxial oblate phase, $\mathrm{N}_{-}^{(2)}$. Note

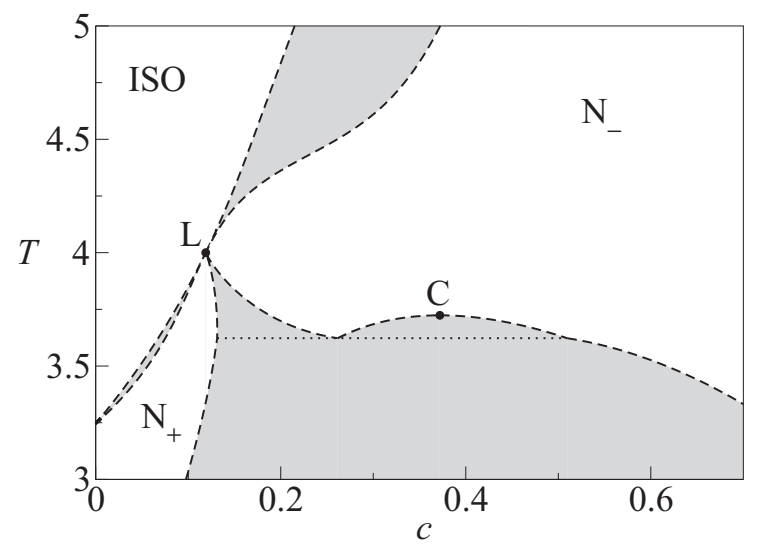

FIG. 9. Phase diagram in terms of temperature $T$ and concentration $c$ of biaxial objects, for degree of biaxiality $\Delta=2.9$. There is no biaxial nematic phase around the Landau point $(\mathrm{L})$. C indicates the simple critical point associated with a coexistence region between two uniaxial oblate phases. The dotted horizontal line indicates a triple point, corresponding to the coexistence of two uniaxial oblate phase and one uniaxial prolate phase.

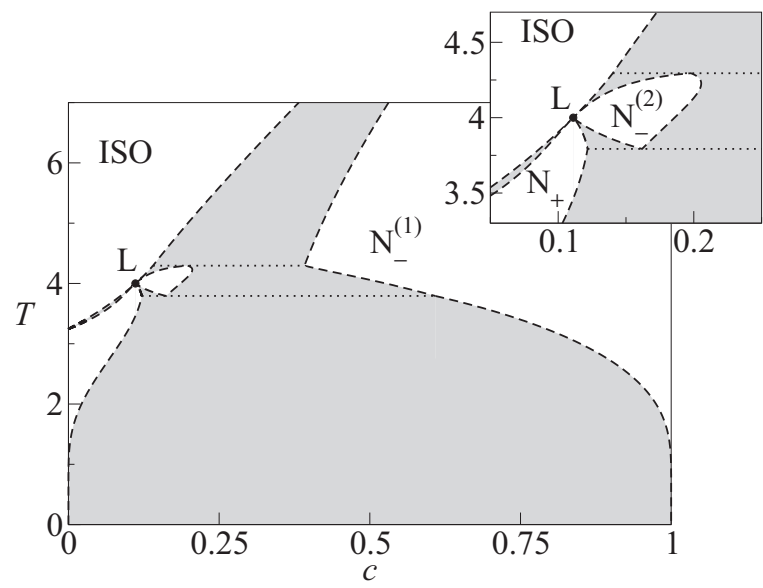

FIG. 10. Phase diagram in terms of temperature $T$ and concentration $c$ of biaxial objects, for degree of biaxiality $\Delta=3.0$. This system corresponds to a mixture of rods and plates with asymmetric interaction energies. A uniaxial nematic prolate phase, $\mathrm{N}_{+}$, and two uniaxial nematic oblate phases, $\mathrm{N}_{-}^{(1)}$ and $\mathrm{N}_{-}^{(2)}$, are present. There are two triple points, indicated by the dotted horizontal tie lines.

that, according to Eq. (2), the value $\Delta=3$ corresponds to a binary mixture of rods and plates with asymmetric interaction energies. A symmetric choice of interaction energies [15] presents a much simpler phase diagram, with single nematic uniaxial prolate and oblate phases, in addition to an isotropic phase, and no triple points.

Figures 11 and 12 represent the phase diagrams for $\Delta=5$ and $\Delta=6$, respectively. In these diagrams the values of concentrations are conveniently rescaled, so that visual effects are improved. Also, note that we introduce some separations just to emphasize the more interesting sectors of these phase diagrams. There is no stable Landau point in either phase diagram. In Fig. 11, the uniaxial phases $\mathrm{N}_{+}$and $\mathrm{N}_{-}$coexist with the isotropic (ISO) phase at a triple point, and a biaxial $\mathrm{B}_{-}$phase remains stable at high concentrations. However, in Fig. 12, there is a coexistence region of $\mathrm{B}_{-}$and isotropic phases, as well as a triple point of coexistence of isotropic, $\mathrm{B}_{-}$, and $\mathrm{N}_{+}$phases. Figures 11 and 12 suggest that the triple

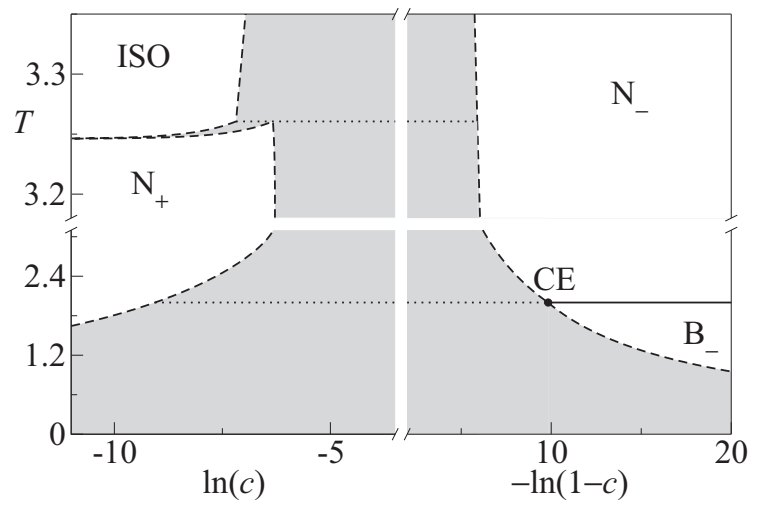

FIG. 11. Phase diagram in terms of temperature $T$ and concentration $c$ of biaxial molecules, for degree of biaxiality $\Delta=5.0$. The Landau point is absent. A triple point appears, at which $\mathrm{N}_{+}, \mathrm{N}_{-}$, and ISO phases coexist. The $\mathrm{B}_{-}$phase is stable at high concentrations. 


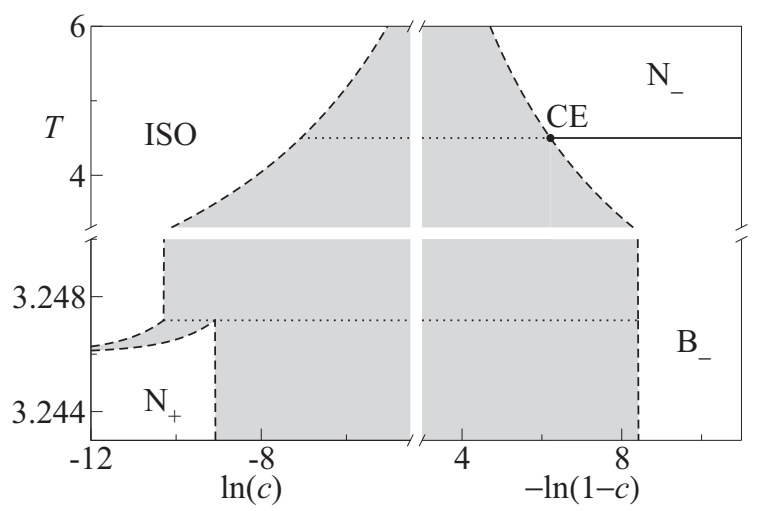

FIG. 12. Phase diagram in terms of temperature $T$ and concentration $c$ of biaxial objects, for degree of biaxiality $\Delta=6.0$. The Landau point is absent. There is a coexistence region between the ISO and the $\mathrm{B}_{-}$phases. In addition, ISO, $\mathrm{B}_{-}$, and $\mathrm{N}_{+}$phases coexist at a triple point. The $\mathrm{B}_{-}$phase is stable at high concentrations.

points meet the critical end point as the degree of biaxiality is increased.

In Fig. 13 we draw the projections of distinct multicritical points on the $\Delta-T$ plane. The line of Landau points is still present, as well as the low-temperature part of the line of critical end points associated with $\mathrm{B}_{-}$. Furthermore, there are lines of triple points associated with the isotropic (ISO) and various nematic uniaxial phases, as depicted in Figs. 9 and 10. The line of Landau points meets the triple lines at another special multicritical point, which we call the Landau end point (LE). This point is characterized by the coexistence of a critical isotropic phase and a noncritical $\mathrm{N}_{-}$phase. Otherwise, two triple lines meet the line of simple critical points at two multicritical end points (MCE). In addition, for $\Delta \simeq 5.5$, the triple line meets the critical end line at a multicritical point, $\mathrm{M}_{2}$, where the critical phase $\mathrm{N}_{-}$coexists with the noncritical $\mathrm{N}_{+}$and isotropic phases. Consequently, for $\Delta \geqslant 5.5$, there is a coexistence region between the isotropic and the $\mathrm{B}_{-}$phases as we increase the concentration of biaxial molecules.

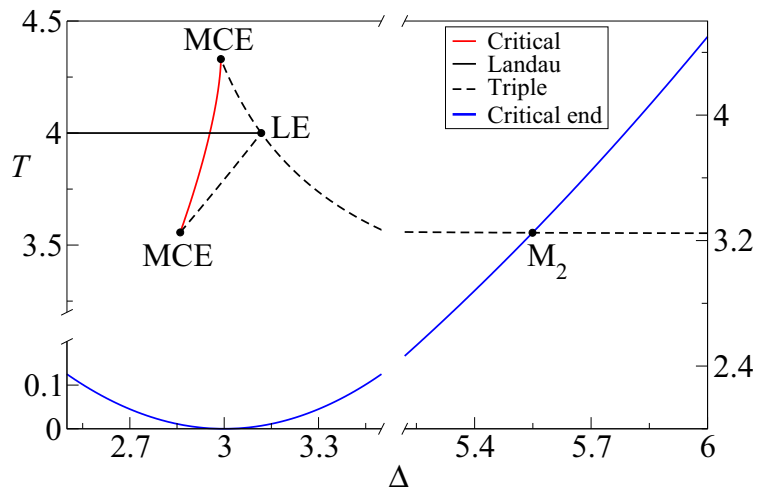

FIG. 13. (Color online) Lines of phase transitions in the $\Delta-T$ plane. Dashed black lines represent lines of triple points. The short solid (red) line of simple critical points ends at two multicritical end points (MCE). The line of Landau points meets the lines of triple points at a Landau end point (LE). Also, the longer (blue) line of critical end points crosses the line of triple points at a multicritical point, $\mathrm{M}_{2}$.

\section{CONCLUSIONS}

We have introduced an elementary six-state Maier-Saupe lattice model, which is obtained by the addition of extra degrees of freedom, of a biaxial nature, to an earlier three-state model. We have then described a phase diagram with biaxial as well as uniaxial nematic structures and an isotropic phase. The fully connected MS6 lattice model, of mean-field character, is sufficiently simple to be amenable to a detailed treatment by standard statistical mechanics techniques. Results are obtained in terms of a parameter $\Delta$ which gauges the degree of biaxiality. We then use this MS6 model to consider a binary mixture of intrinsically uniaxial $(\Delta=0)$ and intrinsically biaxial $(\Delta \neq 0)$ molecules and investigate the effects of "shape variations" on the phase diagrams in terms of temperature and either chemical potential or concentration of biaxial molecules.

Taking into account the fluid character of the liquidcrystalline systems, in the present work we have restricted the analysis to the thermalized (annealed) situation, in which case orientational and shape degrees of freedom reach equilibrium simultaneously. We obtain a wealth of topologically distinct phase diagrams, with several nematically ordered structures and multicritical points.

In the uniform case, in terms of the temperature $T$ and parameter $\Delta$, we regain the first-order transitions between isotropic and uniaxial nematic phases, and the critical lines between the biaxial and the uniaxial nematic phases, which meet at a Landau multicritical point. For a binary mixture of biaxial and uniaxial molecules, we have drawn a number of phase diagrams in terms of the temperature versus concentration of biaxial molecules, with fixed values of $\Delta$, which display many distinct features. Depending on the parameters, additional multicritical points appear, such as tricritical and critical end points associated with the biaxial nematic phase. The Landau, tricritical, and critical end points may give rise to a higher-order multicritical point. Also, depending on the range of values of $\Delta$, there may be a line of Landau points meeting a line of triple points at a Landau end point. This intricate behavior of the mixtures of intrinsically uniaxial and biaxial molecules can be understood in terms of crossings of the microscopic energy levels as we change the degree of biaxiality $\Delta$.

The explicit expressions for the free energy were used to obtain the coefficients of an expansion at high temperatures, in the vicinity of the Landau multicritical point and to make contact with the Landau-de Gennes theory. We were then able to check our numerical findings against a number of phenomenological calculations in the literature. Also, we have provided a simple way of obtaining the expansion coefficients in terms of the values of the molecular parameters.

The calculations predict the presence of stable biaxial nematic phase at low temperatures and sufficiently high concentrations of biaxial molecules, which is in agreement with recent calculations of Longa and coworkers [16] for a more elaborate model system of a mixture of biaxial molecules in the annealed situation. Depending on the degree of biaxiality, we predict first-order transitions between biaxial and uniaxial nematic phases, as well as tricritical points, even in the absence of a Landau multicritical point. For larger values of the degree of biaxiality, the line of Landau points splits into 
lines of triple points. Also, we note a clear reentrance of the biaxial regions for some choices of the parameters, which is in agreement with the early work of Alben on a lattice model of platelets [6].

In some recent publications, Akpinar, Reis, and FigueiredoNeto [29,30] reported X-ray and optical characterizations of biaxial nematic structures in a large class of quaternary liquid-crystalline mixtures. From these measurements, it has been possible to establish many novel phase diagrams in terms of the temperature and molar fraction of the components, which represents a real advance with respect to the early work of Yu and Saupe on a ternary lyotropic mixture. For all concentrations of the amphiphile component, if there is a nematic biaxial structure, it is thermodynamically stable at intermediate temperatures, between regions of different uniaxial nematic structures, at lower and higher temperatures. Also, there are examples of temperature-concentration phase diagrams with a clear indication of the existence of a Landau multicritical point. With a suitable choice of parameters, the MS6 model of binary mixtures can qualitatively reproduce all of these observations. In a very recent experimental investigation, Amaral and coworkers [31] reanalyzed the phase diagram of a ternary sodium dodecyl sulfate lyotropic mixture and pointed out the peculiar coexistence of uniaxial and biaxial nematic structures, which still seems to demand a theoretical explanation.

The present calculations, for the elementary MS6 lattice model at the mean-field level, are a contribution to the understanding of the effects of shape variations on the thermodynamic behavior of complex liquid-crystalline systems. The model of a binary mixture is sufficiently simple to produce a number of analytical and numerical results for a wide range of values of the molecular parameters. Also, it seems to be possible to go beyond the mean-field scenario. The use of more powerful techniques may uncover additional aspects of the phase diagrams, in particular, limitations of the mean-field approach at very low and very high concentrations.

\section{ACKNOWLEDGMENT}

We thank the anonymous referee for useful suggestions. We also acknowledge the financial support of the Brazilian agencies FAPESP and CNPq, as well as that of the Brazilian research funding programs INCT and NAP on Complex Fluids.

\section{APPENDIX: RELATION BETWEEN THE BIAXIALITY PARAMETER $\triangle$ AND THE MOLECULAR ANISOTROPY}

Consider a mesogen which, in a schematic, can be represented by a rectangular parallelepiped with mass $m$ and edges of lengths $a, b$, and $c$. The elements of a "traceless inertia tensor" $\boldsymbol{\Lambda}$ for this object can be defined as

$$
\Lambda_{i j}=I_{i j}-\frac{1}{3} \delta_{i j} \operatorname{Tr} \mathbf{I},
$$

where $\delta_{i j}$ is the Kronecker delta and $\mathbf{I}$ is the inertia tensor.

In diagonal form, $\boldsymbol{\Lambda}$ can be written as

$$
\boldsymbol{\Lambda}=\left(\begin{array}{ccc}
\lambda_{1} & 0 & 0 \\
0 & \lambda_{2} & 0 \\
0 & 0 & \lambda_{3}
\end{array}\right)
$$

with

$$
\begin{aligned}
& \lambda_{1}=\frac{m}{36}\left(-2 a^{2}+b^{2}+c^{2}\right), \\
& \lambda_{2}=\frac{m}{36}\left(a^{2}-2 b^{2}+c^{2}\right), \\
& \lambda_{3}=\frac{m}{36}\left(a^{2}+b^{2}-2 c^{2}\right) .
\end{aligned}
$$

Defining

$$
\Delta=3 \frac{b^{2}-a^{2}}{a^{2}+b^{2}-2 c^{2}},
$$

we can rewrite $\Lambda$ as

$$
\boldsymbol{\Lambda}=\frac{\lambda_{3}}{2}\left(\begin{array}{ccc}
-1+\Delta & 0 & 0 \\
0 & -1-\Delta & 0 \\
0 & 0 & 2
\end{array}\right)
$$

which is proportional to the first diagonal form of the quadrupole tensor in Eq. (2). The other forms would of course be obtained by the other five configurations in which the parallelepiped oriented so that its edges are always parallel to the Cartesian axis.

Assuming $\lambda_{3}<0$, the choice $a=b<c$ would lead to a "rod-like" uniaxial object and to $\Delta=0$, whereas $a=c>b$ would correspond to a "disk-like" uniaxial object, with $\Delta=3$. Both $0<\Delta<3$ and $\Delta>3$ would correspond to unequal edges and, thus, to biaxial objects.
[1] B. R. Acharya, A. Primak, and S. Kumar, Phys. Rev. Lett. 92, 145506 (2004).

[2] L. A. Madsen, T. J. Dingemans, M. Nakata, and E. T. Samulski, Phys. Rev. Lett. 92, 145505 (2004).

[3] K. Merkel, A. Kocot, J. K. Vij, R. Korlacki, G. H. Mehl, and T. Meyer, Phys. Rev. Lett. 93, 237801 (2004).

[4] G. R. Luckhurst, Nature 430, 413 (2004).

[5] M. J. Freiser, Phys. Rev. Lett. 24, 1041 (1970).

[6] R. Alben, Phys. Rev. Lett. 30, 778 (1973).

[7] N. Bocarra, R. Medjani, and L. de Sèze, J. Phys. 38, 149 (1977).

[8] J. P. Straley, Phys. Rev. A 10, 1881 (1974).

[9] G. R. Luckhurst, S. Naemura, T. J. Sluckin, K. S. Thomas, and S. S. Turzi, Phys. Rev. E 85, 031705 (2012).
[10] L. J. Yu and A. Saupe, Phys. Rev. Lett. 45, 1000 (1980).

[11] R. Berardi, L. Muccioli, S. Orlandi, M. Ricci, and C. Zannoni, J. Phys.: Condens. Matter 20, 463101 (2008).

[12] Y. Martinez-Ratón and J. A. Cuesta, Phys. Rev. Lett. 89, 185701 (2002).

[13] E. F. Henriques and V. B. Henriques, J. Chem. Phys. 107, 8036 (1997).

[14] E. do Carmo, D. B. Liarte, and S. R. Salinas, Phys. Rev. E 81, 062701 (2010).

[15] E. do Carmo, A. P. Vieira, and S. R. Salinas, Phys. Rev. E 83, 011701 (2011).

[16] L. Longa, G. Pajak, and T. Wydro, Phys. Rev. E 76, 011703 (2007). 
[17] M. J. de Oliveira and A. M. F. Neto, Phys. Rev. A 34, 3481 (1986).

[18] S. K. Ma, Modern Theory of Critical Phenomena (W. Benjamin, New York, 1976).

[19] P. G. de Gennes and J. Prost, The Physics of Liquid Crystals (Oxford University Press, New York, 1993).

[20] As shown in Refs. [21] and [22], the general form of an anisotropic interaction between biaxial molecules involves two biaxiality parameters. For simplicity, here we deal with a special limit which, in the notation of Ref. [23], corresponds to $\lambda=\gamma^{2}$ and allows us to work with a single biaxiality parameter.

[21] G. R. Luckhurst et al., Mol. Phys. 30, 1345 (1975).

[22] G. R. Luckhurst and S. Romano, Mol. Phys. 40, 129 (1980).

[23] A. M. Sonnet, E. G. Virga, and G. E. Durand, Phys. Rev. E 67, 061701 (2003).

[24] T. A. Witten and P. Pincus, Structural Fluids: Polymers, Colloids, Surfactants (Oxford University Press, New York, 2004), Chap. 2.

[25] The parameters $S$ and $\eta$ introduced in Eq. (13) are, respectively, proportional to the parameters $q$ and $\eta$ defined in Eqs. (2.68) and (2.69) in Ref. [19]. In our case, comparison between Eqs. (2) and (13) indicates that $S$ ranges from -1 to 2 , while $\eta$ ranges from $\min (-| \pm S-4|,-2-2 \Delta-S)$ to $\max (|4 \pm S|, 2+2 \Delta+S)$. There has actually been a plethora of notations introduced in the last decades to denote second-rank orientational order parameters for biaxial nematics, as reviewed in Ref. [26]. In particular, it can be shown (see p. 746 in Ref. [26]) that our parameters $S$ and $\eta$ (which are essentially parameters $a$ and $b$ in Ref. [26]) correspond to linear combinations of the four scalar order parameters $S, D, P$, and $C$ (see Ref. [9]) built from a Cartesian representation of the ordering supertensor. Specifically, our $S$ is built from a linear combination of $S$ and $U$ in Ref. [9], while our $\eta$ is built from a linear combination of $P$ and $C$, with the prefactor of $C$ in the combination being proportional to our $\Delta$. (Note that Ref. [26] adopts the notation $S, U, P$, and $F$ for what are essentially the parameters $S, D, P$, and $C$ in Ref. [9].)

[26] R. Rosso, Liq. Cryst. 34, 737 (2007).

[27] E. F. Gramsbergen, L. Longa, and W. H. de Jeu, Phys. Rep. 135, 195 (1986)

[28] X. Zheng and P. Palffy-Murhoray, Discrete Cont. Dynam. Syst. B 15, 475 (2011).

[29] E. Akpinar, D. Reis, and A. M. Figueiredo Neto, Eur. Phys. J. E 35, 50 (2012).

[30] E. Akpinar, D. Reis, and A. M. Figueiredo Neto, Liq. Cryst. 39, 881 (2012).

[31] L. Q. Amaral, O. R. Santos, W. S. Braga, N. M. Kimura, and A. J. Palangana, Liq. Cryst. 42, 240 (2015). 


\title{
Density functional theory for dense nematic liquid crystals with steric interactions
}

\author{
Eduardo S. Nascimento* and Peter Palffy-Muhoray ${ }^{\dagger}$ \\ Liquid Crystal Institute, Kent State University, Kent, Ohio 44240, USA \\ Jamie M. Taylor ${ }^{\ddagger}$ and Epifanio G. Virga ${ }^{\S}$ \\ Mathematical Institute, University of Oxford, Oxford OX2 6GG, United Kingdom \\ Xiaoyu Zheng" \\ Department of Mathematical Sciences, Kent State University, Kent, Ohio 44240, USA
}

(Received 27 February 2017; revised manuscript received 21 May 2017; published 7 August 2017)

\begin{abstract}
The celebrated work of Onsager on hard particle systems, based on the truncated second order virial expansion, is valid at relatively low volume fractions for large aspect ratio particles. While it predicts the isotropic-nematic phase transition, it does not provide a realistic equation of state in that the pressure remains finite for arbitrarily high densities. In this work, we derive a mean field density functional form of the Helmholtz free energy for nematics with hard core repulsion. In addition to predicting the isotropic-nematic transition, the model provides a more realistic equation of state. The energy landscape is much richer, and the orientational probability distribution function in the nematic phase possesses a unique feature-it vanishes on a nonzero measure set in orientation space.
\end{abstract}

DOI: 10.1103/PhysRevE.96.022704

\section{INTRODUCTION}

An ensemble of nonspherical particles, interacting via hard core interactions, exhibits a first order isotropic-nematic phase transition as the number density increases, followed by a nematic-solid transition [1,2] (see also the companion paper [3] for a number of technical results on the excluded volume of ellipsoids). In his celebrated theoretical work [4], Onsager successfully predicted the isotropic-nematic transition. Using the canonical ensemble, he expressed the free energy in terms of the orientational probability density function $f(\hat{\mathbf{l}})$, where $\hat{\mathbf{l}} \in \mathbb{S}^{2}$ is a unit vector along the symmetry axis of a particle. Truncating the virial expansion after the second term, in the low volume fraction limit, the free energy is of the form

$$
\begin{aligned}
F= & N k T\left\{\ln \rho_{0}+\int_{\mathbb{S}^{2}} f(\hat{\mathbf{l}})\right. \\
& \left.\times\left(\ln f(\hat{\mathbf{l}})+\frac{1}{2} \rho_{0} \int_{\mathbb{S}^{2}} f\left(\hat{\mathbf{l}}^{\prime}\right) V_{\mathrm{exc}}\left(\hat{\mathbf{l}}, \hat{\mathbf{I}}^{\prime}\right) d \hat{\mathbf{l}}^{\prime}\right) d \hat{\mathbf{l}}\right\},
\end{aligned}
$$

where $N$ is the number of particles, $k$ is Boltzmann's constant, $T$ is the temperature, and $\rho_{0}$ is the number density. ${ }^{1}$ In addition to the isotropic-nematic transition, the model describes phase separation and the coexistence of nematic and isotropic phases. In spite of its many successes [6], the theory has a major

\footnotetext{
*enascime@kent.edu

†mpalffy@kent.edu

†Jamie.Taylor@maths.ox.ac.uk

${ }^{\S}$ On leave from: Dipartimento di Matematica, Università di Pavia,

Via Ferrata 5, I-27100 Pavia, Italy; eg.virga@unipv.it

"zheng@math.kent.edu

${ }^{1}$ A justification of Eq. (1) can also be given in terms of a reduced cluster expansion, based only on Penrose's spanning trees, which explains the success of Onsager's theory better than the omission of all terms in the virial expansion but the first [5].
}

limitation: it does not provide a reasonable description of the behavior near the dense packing limit. It does not give a reasonable equation of state; the pressure remains finite at arbitrarily high number densities.

Onsager's theory was rooted in the Mayer expansion, which formed the basis of Mayer's statistical mechanics book [7]. Seen in the light of the full Mayer expansion (and the related problem of its convergence) the truncation at the first power in the number density in Eq. (1) appears unjustified. Many attempts have been made to explore the limits of validity of the Onsager theory and to propose extensions.

Straley [8,9], by combining analytical and numerical methods, estimated the third virial coefficient for hard rods and concluded that Onsager's theory would not be quantitatively accurate for values of the length-to-breadth ratio $\kappa$ less than 100 , a prediction that was far too pessimistic and that was indeed proven to be unrealistic by the Monte Carlo simulations of Frenkel and Mulder [1,2] with ellipsoids of revolution. They showed that an ordering transition does take place already for ellipsoids of aspect ratio $\kappa=3$, though the densities involved could be too high to be compatible with the Onsager theory. It then became clear that it is not the degree of anisometry of the particles, but their density that may more easily challenge the validity of Onsager's theory. The emphasis thus shifted from the particles' shape to their filling fraction. A similar conclusion was drawn much later in the work of Tjipto-Margo and Evans [10], who included the third virial coefficient in the theory for ellipsoids. For aspect ratios $\kappa>5$, their extended theory predicts the correct variation of the order parameter with density and is in agreement with simulations.

A valuable improvement to the Mayer paradigm of density expansion was introduced by Barboy and Gilbert [11]. They remarked that an expansion in the variable $y=\eta /(1-\eta)$, where $\eta$ denotes the volume fraction, has better convergence properties than the usual series in the number density. Their theory was applied to dumbbells and spherocylinders in the isotropic phase [11], as well as to hard parallelepipeds [12], but 
in the restricted-orientation approximation of Zwanzig [13]. No clear superiority of this theory to the classical Mayer theory was ever established with certainty [6].

A considerable step towards the extension of Mayer theory was taken by Parsons [14] and Lee [15], who moved along different lines of thought but arrived at one and the same theory. In Lee's interpretation, the emphasis is on the equation of state, which is modeled after the theory of Carnahan and Starling [16] for real gases of hard spheres. The virial coefficients for the pressure expansion of Mayer's theory are rescaled to those hypothesized by Carnahan and Starling in their extrapolation of the viral coefficients for hard spheres known at that time. As a consequence of this rescaling, the expansion for the free energy functional is resummed exactly and expressed in the form of a modified Onsager bilinear functional in the probability distribution density. Lee applied this theory to a fluid of axially symmetric ellipsoids and found it in a better agreement with simulations than other theories available at that time [17]. An alternative theory was independently proposed by Baus and co-workers [18,19], which despite appearances is easily seen to reduce to the Parsons-Lee theory [6].

As successful as the Parsons-Lee theory may be, it is limited by being a resummed version of the Mayer theory, and as such it remains exposed to all the unresolved issues concerned with the convergence of the underlying Mayer power series. We therefore attempt here to go beyond the Mayer paradigm.

In this paper, the density functional theory of aspherical hard particles is revisited. We are particularly interested in the phase behavior of hard ellipsoids at high densities, and near the densest packing limit. This very system will be used as a test case for the theory. Within the comfort zone of Mayer expansion, the evaluation of the partition function at high densities, even for hard spheres, has eluded researchers to date in spite of considerable effort. Here we take the simplest approach to explore orientational order in this regime. To clearly focus on this problem, we have chosen a minimal model, essentially at the van der Waals level. Although one might suspect that this form cannot provide quantitative predictions, our comparison with simulations for ellipsoids is encouraging. More generally, we feel that, in spite of its simplicity, it can give valuable insights into the consequences of the salient aspect of the problem: the depletion of available phase space as the density is increased.

A major challenge is the determination of the orientational distribution function subject to the hard constraint that the number of available states to the system is positive. This constraint is not always strictly enforced in the literature [20]. One striking result of this constraint, in the mean field limit, is that at densities above the isotropic-nematic transition, the orientational distribution function which minimizes the free energy has a compact support over orientation space. We also find that the nematic phase is more orientationally ordered than in the Onsager theory at the same density, and the density of the coexisting isotropic and nematic phases is a simple function of the particle shape. Our simple model gives a reasonable equation of state; that is, the pressure diverges at the dense packing limit.

The goal of the paper is to provide an approximate but near realistic description of dense orientationally ordered hard particle systems; currently available descriptions are either low density approximations [21,22], or they rely on somewhat ad hoc Carnahan-Starling-type corrections to the free energy at high densities [23,24].

In Sec. II, we derive our theory. In Sec. III, we write the free energy for a system of hard ellipsoids with arbitrary number density, derive and solve the Euler-Lagrange equation for the probability density function, and arrive at the equation of state. In Sec. IV, we assume uniaxial nematic order and present some numerical results relevant to this case. In particular, in Sec. IV C, we discuss phase separation and two-phase coexistence. In Sec. V, we explore the possibility of biaxial equilibrium phases. Section VI is devoted to a close comparison with simulations. Finally, in Sec. VII we draw the conclusions of this study and summarize our results. The paper is closed by an Appendix, where we justify the approximate expression used in Sec. III for the excluded volume of ellipsoids of revolution.

\section{THEORY}

We consider a one-component system consisting of hard particles. For simplicity, we omit attractive interactions. The configurational Helmholtz free energy of a system of $N$ particles, within an additive constant, is

$$
F=-k T \ln \frac{1}{N !} \int_{\Omega^{N}} e^{-\frac{1}{k T} \sum_{1 \leqslant i<j \leqslant N} U_{i j}^{R}} d \mathbf{q}_{1} \cdots d \mathbf{q}_{N},
$$

where $\mathbf{q}_{i}$ is the generalized (orientational and positional) coordinate of the $i$ th particle, and $U_{i j}^{R}=U^{R}\left(\mathbf{q}_{i}, \mathbf{q}_{j}\right)$ is the repulsive interaction energy between particles $i$ and $j$; the sum is over all pairs of particles. Explicitly, the interaction potential is

$$
U^{R}\left(\mathbf{q}_{i}, \mathbf{q}_{j}\right)= \begin{cases}\infty & \text { if particles interpenetrate, } \\ 0 & \text { otherwise. }\end{cases}
$$

We write

$$
G_{N}=\int_{\Omega^{N}} e^{-\frac{1}{k T} \Sigma_{1 \leqslant i<j \leqslant N} U_{i j}^{R}} d \mathbf{q}_{1} \cdots d \mathbf{q}_{N},
$$

where the quantity $G_{N}$ can be thought of as the number of states available to $N$ distinguishable particles. We consider adding one particle to the system. Then

$$
\begin{aligned}
G_{N+1}= & \int_{\Omega^{N}} e^{-\frac{1}{k T} \Sigma_{1 \leqslant i<j \leqslant N} U_{i j}^{R}}\left(\int_{\Omega} e^{-\frac{1}{k T} \Sigma_{i=1}^{N} U_{i(N+1)}^{R}} d \mathbf{q}_{N+1}\right) \\
& \times d \mathbf{q}_{1} \cdots d \mathbf{q}_{N},
\end{aligned}
$$

and, since the probability density function of a given configuration is

$$
P\left(\mathbf{q}_{1}, \cdots, \mathbf{q}_{N}\right)=\frac{e^{-\frac{1}{k T} \Sigma_{1 \leqslant i<j \leqslant N} U_{i j}^{R}}}{G_{N}},
$$

we have

$$
\begin{aligned}
G_{N+1}= & G_{N} \int_{\Omega^{N}} P\left(\mathbf{q}_{1}, \cdots, \mathbf{q}_{N}\right)\left(\int_{\Omega} e^{-\frac{1}{k T} \Sigma_{i=1}^{N} U_{i(N+1)}^{R}} d \mathbf{q}_{N+1}\right) \\
& \times d \mathbf{q}_{1} \cdots d \mathbf{q}_{N} \\
= & G_{N}\left\langle\int_{\Omega} e^{-\frac{1}{k T} \Sigma_{i=1}^{N} U_{i(N+1)}^{R}} d \mathbf{q}_{N+1}\right\rangle
\end{aligned}
$$


where the average is computed relative to $P$. Since all particles are equivalent, we may write

$$
G_{N}=\left\langle\int_{\Omega} e^{-\frac{1}{k T} \Sigma_{i=2}^{N} U_{1 i}^{R}} d \mathbf{q}_{1}\right\rangle^{N},
$$

where the integral represents the average free volume per particle in an ensemble of $N$ particles. We can equivalently write this as

$$
\begin{aligned}
G_{N} & =\left\langle\int_{\Omega} 1-\left(1-e^{-\frac{1}{k T} \Sigma_{i=2}^{N} U_{1 i}^{R}}\right) d \mathbf{q}_{1}\right\rangle^{N} \\
& =\left(\int_{\Omega}\left[1-W\left(\mathbf{q}_{1}\right)\right] d \mathbf{q}_{1}\right)^{N},
\end{aligned}
$$

where

$$
W\left(\mathbf{q}_{1}\right)=\left\langle 1-e^{-\frac{1}{k T} \Sigma_{i=2}^{N} U_{1 i}^{R}}\right\rangle
$$

is the average excluded volume fraction.

The free energy then becomes

$$
F=-k T \ln \frac{1}{N !}\left(\int_{\Omega}\left[1-W\left(\mathbf{q}_{1}\right)\right] d \mathbf{q}_{1}\right)^{N} .
$$

This important result is exact. The integrand in Eq. (11) can be regarded as the fraction of the total volume available to particle 1 , or the average free volume fraction. We next write that

$$
W\left(\mathbf{q}_{1}\right)=\frac{N}{V} v_{\text {eff }},
$$

where $v_{\text {eff }}$ is the average volume effectively occupied by one particle. For two hard spheres of volume $v_{0}$, the pair excluded volume $V_{\text {exc }}$ is

$$
V_{\mathrm{exc}}=8 v_{0},
$$

and for $N=2$, Eq. (10) gives exactly

$$
v_{\text {eff }}=\frac{1}{2} V_{\text {exc }} \text {. }
$$

This is likely to remain a good approximation in the low density limit, as used in the van der Waals equation [25], pp. 90-91, but it is clear from Eq. (10) as well as from experiments that at high densities $v_{\text {eff }}$ is reduced considerably from this value. For close packed spheres, for example,

$$
v_{\mathrm{eff}}=\frac{3}{4 \pi \sqrt{2}} V_{\mathrm{exc}} \simeq \frac{1}{6} V_{\mathrm{exc}} .
$$

We write therefore that

$$
v_{\text {eff }}=\lambda V_{\text {exc }},
$$

where $\lambda$ is a parameter, taken to be nearly constant in our high density limit, to be determined by comparison with experiment. ${ }^{2}$ This justifies the following postulate of our theory:

$$
W\left(\mathbf{q}_{1}\right)=\lambda \int_{\Omega} \rho\left(\mathbf{q}_{2}\right)\left(1-e^{-\frac{U^{R}\left(\mathbf{q}_{1}, \mathbf{q}_{2}\right)}{k T}}\right) d \mathbf{q}_{2},
$$

\footnotetext{
${ }^{2}$ This parameter $\lambda$ is not dissimilar from the coordination parameter $z$ introduced in the generalization presented in [26] of the classical mean field theory as incarnated in [27] (see also Chap. 1 of [28] for a more detailed discussion).
}

where $\rho(\mathbf{q})$ is the number density of particles with generalized coordinate q. More generally, in Eq. (17) $\lambda$ should be thought of as a function of the number density $\rho\left(\mathbf{q}_{1}\right)$, for whose form we have yet to present a firm first-principles derivation. Here, $\lambda$ will be treated as an adjustable parameter; comparison with simulations will indicate the range within which the function $\lambda(\rho)$ is expected to vary.

To obtain the density functional form of the free energy, we assume that the density $\rho\left(\mathbf{q}_{i}\right)$ is a slowly varying function of $\mathbf{q}_{i}$. We consider an element $\Omega_{i}$ of phase space, containing $N_{i}$ particles and having volume $\Delta \mathbf{q}_{i}$, sufficiently small so that $\rho\left(\mathbf{q}_{i}\right)$ in element $\Omega_{i}$ is nearly constant. The free energy $F_{i}$ of region $\Omega_{i}$ is of the form of Eq. (11); that is, by Stirling's approximation, ${ }^{3}$

$$
F_{i}=-k T \ln \left(\frac{1}{N_{i}} \int_{\Omega_{i}}\left[1-W\left(\mathbf{q}_{i}\right)\right] d \mathbf{q}_{i}\right)^{N_{i}},
$$

and since $\rho\left(\mathbf{q}_{i}\right)$ is nearly constant, we have that $N_{i}=$ $\rho\left(\mathbf{q}_{i}\right) \Delta \mathbf{q}_{i}$, and

$$
F_{i}=-k T \ln \left(\frac{1}{\rho\left(\mathbf{q}_{i}\right)}\left[1-W\left(\mathbf{q}_{i}\right)\right]\right)^{\rho\left(\mathbf{q}_{i}\right) \Delta \mathbf{q}_{i}} .
$$

Then, since the free energy is additive, we can write for the entire system

$$
F=-k T \ln \prod_{i}\left(\frac{1}{\rho\left(\mathbf{q}_{i}\right)}\left[1-W\left(\mathbf{q}_{i}\right)\right]\right)^{\rho\left(\mathbf{q}_{i}\right) \Delta \mathbf{q}_{i}},
$$

or, passing to the continuum limit,

$$
F=k T \int_{\Omega} \rho(\mathbf{q}) \ln \rho(\mathbf{q}) d \mathbf{q}-k T \int_{\Omega} \rho(\mathbf{q}) \ln [1-W(\mathbf{q})] d \mathbf{q},
$$

which is the standard density functional form. Explicitly, this is

$$
\begin{aligned}
F= & k T \int_{\Omega} \rho\left(\mathbf{q}_{1}\right) \ln \rho\left(\mathbf{q}_{1}\right) d \mathbf{q}_{1}-k T \int_{\Omega} \rho\left(\mathbf{q}_{1}\right) \\
& \times \ln \left[1-\lambda \int_{\Omega} \rho\left(\mathbf{q}_{2}\right)\left(1-e^{-\frac{U^{R}\left(\mathbf{q}_{1}, \mathbf{q}_{2}\right)}{k T}}\right) d \mathbf{q}_{2}\right] d \mathbf{q}_{1} .
\end{aligned}
$$

For our problem, it is convenient to write the generalized coordinates in terms of position and orientation; then

$$
\begin{aligned}
F= & k T \int_{\mathbb{S}^{2}} \int_{\mathcal{B}} \rho\left(\mathbf{r}_{1}, \hat{\mathbf{l}}_{1}\right) \ln \rho\left(\mathbf{r}_{1}, \hat{\mathbf{l}}_{1}\right) d^{3} \mathbf{r}_{1} d^{2} \hat{\mathbf{l}}_{1} \\
& -k T \int_{\mathbb{S}^{2}} \int_{\mathcal{B}} \rho\left(\mathbf{r}_{1}, \hat{\mathbf{l}}_{1}\right) \ln \left[1-\lambda \int_{\mathbb{S}^{2}} \int_{\mathcal{B}} \rho\left(\mathbf{r}_{2}, \hat{\mathbf{l}}_{2}\right)\right. \\
& \left.\times\left(1-e^{-\frac{U^{R}\left(\mathbf{r}_{1}, \hat{\mathbf{l}}_{1}, \mathbf{r}_{2}, \hat{\mathbf{l}}_{2}\right)}{k T}}\right) d^{3} \mathbf{r}_{2} d^{2} \hat{\mathbf{l}}_{2}\right] d^{3} \mathbf{r}_{1} d^{2} \hat{\mathbf{l}}_{1},
\end{aligned}
$$

where $\rho(\mathbf{r}, \hat{\mathbf{l}})$ is the number density of particles with center of mass at position $\mathbf{r}$, and orientation of symmetry axis along $\hat{\mathbf{l}}$. The unit sphere $\mathbb{S}^{2}$ is the orientation space. The region

\footnotetext{
${ }^{3}$ To be precise, the term $-k T N_{i}$ has been omitted on the right-hand side of Eq. (18). Its inclusion would only add a term proportional to the total number of particles to the right-hand side of Eq. (21).
} 
$\mathcal{B}$ in physical space denotes the position space, occupied by particles. In a homogeneous system, the density is independent of $\mathbf{r}$, so we write

$$
\rho(\mathbf{r}, \hat{\mathbf{l}})=\rho_{0} f(\hat{\mathbf{I}}),
$$

where $\rho_{0}$ is now simply the number density of particles, and $f(\hat{\mathbf{I}})$ is the single particle orientational distribution function satisfying

$$
\int_{\mathbb{S}^{2}} f(\hat{\mathbf{l}}) d^{2} \hat{\mathbf{l}}=1
$$

Integrating over $\mathbf{r}_{2}$ in Eq. (23) gives

$$
\begin{gathered}
\int_{\mathbb{S}^{2}} \int_{\mathcal{B}} \rho_{0} f\left(\hat{\mathbf{l}}_{2}\right)\left(1-e^{-\frac{U^{R}\left(\mathbf{r}_{1}, \hat{\mathbf{l}}_{1}, \mathbf{r}_{2}, \hat{\mathbf{l}}_{\mathbf{2}}\right)}{k T}}\right) d^{3} \mathbf{r}_{2} d^{2} \hat{\mathbf{l}}_{2} \\
=\rho_{0} \int_{\mathbb{S}^{2}} f\left(\hat{\mathbf{l}}_{2}\right) V_{\mathrm{exc}}\left(\hat{\mathbf{l}}_{1}, \hat{\mathbf{l}}_{2}\right) d^{2} \hat{\mathbf{l}}_{2},
\end{gathered}
$$

where

$$
V_{\mathrm{exc}}\left(\hat{\mathbf{l}}_{1}, \hat{\mathbf{l}}_{2}\right)=\int_{\mathcal{B}}\left(1-e^{-\frac{U^{R}\left(\mathbf{r}_{1}, \hat{\mathbf{l}}_{1}, \mathbf{r}_{2}, \hat{\mathbf{r}}_{2}\right)}{k T}}\right) d^{3} \mathbf{r}
$$

is the excluded volume of two particles, which depends only on the relative orientation of the particles with respect to one another. The free energy in Eq. (22) then becomes

$$
\begin{aligned}
F= & k T \rho_{0} V\left[\ln \rho_{0}+\int_{\mathbb{S}^{2}} f\left(\hat{\mathbf{l}}_{1}\right) \ln f\left(\hat{\mathbf{l}}_{1}\right) d^{2} \hat{\mathbf{l}}_{1}-\int_{\mathbb{S}^{2}} f\left(\hat{\mathbf{l}}_{1}\right)\right. \\
& \left.\times \ln \left(1-\lambda \rho_{0} \int_{\mathbb{S}^{2}} f(\hat{\mathbf{l}}) V_{\mathrm{exc}}\left(\hat{\mathbf{l}}_{1}, \hat{\mathbf{l}}_{2}\right) d^{2} \hat{\mathbf{l}}_{2}\right) d^{2} \hat{\mathbf{l}}_{1}\right] .
\end{aligned}
$$

If $\rho_{0}$ is small, one can expand the logarithm and recover the theory of Onsager [4] as well as that of Doi and Edwards [29], p. $354 .{ }^{4}$ We note that the expansion is only valid when

$$
\lambda \rho_{0} \int_{\mathbb{S}^{2}} f\left(\hat{\mathbf{l}}_{2}\right) V_{\mathrm{exc}}\left(\hat{\mathbf{l}}_{1}, \hat{\mathbf{l}}_{2}\right) d^{2} \hat{\mathbf{l}}_{2}<1 .
$$

In general, the argument of the logarithm must be positive. The equilibrium orientational distribution $f(\hat{\mathbf{l}})$ is a function of $\rho_{0}$, and is not known a priori. Intuitively, one would expect particles to align more and more as the number density is increased, corresponding to a decrease of the orientationally averaged excluded volume with number density. It follows that the densest packing density cannot be greater than the inverse of the smallest average excluded volume of a pair of particles. The orientational distribution function is therefore expected to depend sensitively on the number density $\rho_{0}$.

To capture the phase behavior in the high density regime, we keep the full logarithmic dependence in Eq. (28); this is the salient feature of our approach. This results in a remarkable phenomenon: above a critical value of $\rho_{0}$, the equilibrium distribution function $f(\hat{\mathbf{I}})$ is continuous over the whole orientation space $\mathbb{S}^{2}$, but vanishes on a region with positive measure; that is, at high densities, some regions of orientation space are not accessible to particles.

${ }^{4}$ For a gas of hard spheres, van Kampen [30] had already proposed a free energy that features the logarithm of the free volume. However, his derivation, which admittedly follows closely the unpublished work of Ornstein (1908), is ad hoc and finds implicit inspiration in the exact treatment of a one dimensional Tonks gas [31].

\section{APPLICATION TO ELLIPSOIDS}

In this section we apply the theory presented in Sec. II to a system of hard ellipsoids of revolution. To this end, our first task is to obtain a simple but reliable expression for the excluded volume of two such particles.

\section{A. Excluded volume}

For identical hard ellipsoids of revolution of length $L$ and width $W$, the volume is $v_{0}=\frac{1}{6} \pi L^{3} / \kappa^{2}$, where $\kappa=L / W$ is the aspect ratio. A simple approximate expression for the excluded volume is derived in the Appendix, where its accuracy is also assessed; it reads as

$$
\begin{aligned}
V_{\mathrm{exc}}\left(\hat{\mathbf{l}}_{1}, \hat{\mathbf{l}}_{2}\right) & =C-\frac{2}{3} D\left(\sigma\left(\hat{\mathbf{l}}_{1}\right): \sigma\left(\hat{\mathbf{l}}_{2}\right)\right) \\
& =C-D P_{2}\left(\hat{\mathbf{l}}_{1} \cdot \hat{\mathbf{l}}_{2}\right), \\
C & =8 v_{0}(1+\varphi), \\
D & =8 v_{0} \varphi,
\end{aligned}
$$

where $\sigma(\hat{\mathbf{I}})=\frac{1}{2}(3 \hat{\mathbf{l}}-\mathbf{I})$ denotes the orientation descriptor of a particle with symmetry axis oriented along $\hat{\mathbf{I}}$ and $P_{2}(x)=$ $\frac{3}{2} x^{2}-\frac{1}{2}$ is the second Legendre polynomial. The shape parameters $C \geqslant 0$ and $D<C$ are expressed in terms of a convenient measure of the anisotropy measured by $\varphi$, which in Eq. (A7) is given as an explicit, though complicated, positive function of the eccentricity $\epsilon$ of the ellipsoid, defined as

$$
\epsilon:= \begin{cases}\sqrt{1-\kappa^{2}} & \text { for } 0 \leqslant \kappa \leqslant 1 \\ \sqrt{1-\frac{1}{\kappa^{2}}} & \text { for } \kappa \geqslant 1\end{cases}
$$

For simplicity, we absorb $\lambda$ into $C$ and $D$ and we write

$$
\lambda V_{\operatorname{exc}}\left(\hat{\mathbf{l}}_{1}, \hat{\mathbf{l}}_{2}\right)=c-\frac{2 d}{3}\left(\boldsymbol{\sigma}\left(\hat{\mathbf{l}}_{1}\right): \sigma\left(\hat{\mathbf{l}}_{2}\right)\right)
$$

where $c=\lambda C$ and $d=\lambda D$.

Substitution of the expression in Eq. (34) for the excluded volume into Eq. (28) gives for the free energy density (per unit volume)

$$
\begin{aligned}
\mathcal{F}= & k T \rho_{0}\left\{\ln \rho_{0}+\int_{\mathbb{S}^{2}} f(\hat{\mathbf{l}}) \ln f(\hat{\mathbf{l}}) d^{2} \hat{\mathbf{I}}-\int_{\mathbb{S}^{2}} f(\hat{\mathbf{l}})\right. \\
& \left.\times \ln \left[1-\rho_{0}\left(c-d \frac{2}{3} \boldsymbol{\sigma}(\hat{\mathbf{l}}): \mathbf{Q}\right)\right] d^{2} \hat{\mathbf{l}}\right\}
\end{aligned}
$$

where

$$
\mathbf{Q}=\int_{\mathbb{S}^{2}} \boldsymbol{\sigma}(\hat{\mathbf{l}}) f(\hat{\mathbf{l}}) d^{2} \hat{\mathbf{l}}=\langle\boldsymbol{\sigma}(\hat{\mathbf{l}})\rangle
$$

is the symmetric and traceless tensor orientational order parameter. The eigenvectors of $\mathbf{Q}$ indicate the principal directions of average orientation, and the eigenvalues provide a measure of the degree of order in the direction along the corresponding eigenvector. The eigenvalues range from $-\frac{1}{2}$ to 
1. ${ }^{5}$ Note that $f(\hat{\mathbf{l}}) \geqslant 0$ is admissible if

$$
1-\rho_{0}\left(c-d \frac{2}{3} \boldsymbol{\sigma}(\hat{\mathbf{I}}): \mathbf{Q}\right)>0,
$$

that is, if the argument of the logarithm is positive.

\section{B. Parametrization}

It is convenient to introduce the dimensionless parameter

$$
\phi=\frac{\rho_{0} c-1}{\rho_{0} d} \in(-\infty, 1],
$$

which is an increasing function of number density $\rho_{0}=$ $\frac{1}{c-d \phi}$. The upper limit for $\phi$ guarantees that inequality (37) is satisfied at least in the case of full alignment. In the very dilute limit, $\rho_{0} \rightarrow 0^{+}, \phi \rightarrow-\infty$; in the dense packing limit, $\rho_{0} \rightarrow \rho_{0 \max }=\frac{1}{c-d}^{-}, \phi \rightarrow 1^{-}$, which corresponds to the densest packing fraction with only pairwise interactions. The free energy density can be written in terms of $\phi$ as

$$
\begin{aligned}
\mathcal{F}= & k T \rho_{0} \int_{\mathbb{S}^{2}}\{f(\hat{\mathbf{I}}) \ln f(\hat{\mathbf{l}})-f(\hat{\mathbf{I}}) \ln [(1-\phi) \\
& \left.\left.-\left(1-\frac{2}{3} \boldsymbol{\sigma}(\hat{\mathbf{l}}): \mathbf{Q}\right)\right]\right\} d^{2} \hat{\mathbf{l}},
\end{aligned}
$$

where we have neglected the additive constant $-k T \rho_{0} \ln d$. To understand the physical significance of $\phi$, we note that we can write

$$
1-\phi=\frac{1}{\varphi}\left(\frac{\rho_{0 \max }}{\rho_{0}}-1\right)
$$

or

$$
1-\phi=\frac{1}{\varphi}\left(\frac{v}{v_{\min }}-1\right),
$$

where $v=1 / \rho_{0}$ is the volume per particle, and $v_{\min }=$
$1 / \rho_{0 \max }$ is the minimum volume per particle. We regard the quantity $1-\phi$ as the the orientational relative free volume, which provides a dimensionless measure of the volume or, equivalently, of the number of states available for orientation. If the particles are spheres, the anisotropy $\varphi$ vanishes and the number of available states diverges. As the anisotropy $\varphi$ is increased, the number of available orientational states decreases, and the system is expected to become more and more ordered. Indeed, this is what happens, as shown below.

\section{Minimization of the free energy}

We next minimize the free energy density with respect to the orientational distribution function $f(\hat{\mathbf{l}})$. We write $\mathcal{F}$ explicitly in terms of $f(\hat{\mathbf{l}})$,

$$
\begin{aligned}
\mathcal{F}= & k T \rho_{0} \int_{\mathbb{S}^{2}}\left[f\left(\hat{\mathbf{l}}_{1}\right) \ln f\left(\hat{\mathbf{l}}_{1}\right)-f\left(\hat{\mathbf{l}}_{1}\right)\right. \\
& \left.\times \ln \left(\frac{2}{3} \boldsymbol{\sigma}\left(\hat{\mathbf{l}}_{1}\right): \int_{\mathbb{S}^{2}} \boldsymbol{\sigma}\left(\hat{\mathbf{l}}_{2}\right) f\left(\hat{\mathbf{l}}_{2}\right) d^{2} \hat{\mathbf{l}}_{2}-\phi\right)\right] d^{2} \hat{\mathbf{l}}_{1},
\end{aligned}
$$

where we have labeled the arguments for clarity.

We have two constraints: the distribution function must be normalized to unity, that is,

$$
\int_{\mathbb{S}^{2}} f(\hat{\mathbf{l}}) d^{2} \hat{\mathbf{I}}=1
$$

and the argument of the logarithm, the free volume fraction, must be positive; that is,

$$
\frac{2}{3} \sigma(\hat{\mathbf{l}}): \mathbf{Q}-\phi>0
$$

Setting formally the first variation to zero gives

$$
\ln f\left(\hat{\mathbf{l}}_{1}\right)+(\mu+1)-\ln \left(\frac{2}{3} \sigma\left(\hat{\mathbf{l}}_{1}\right): \int_{\mathbb{S}^{2}} \boldsymbol{\sigma}\left(\hat{\mathbf{l}}_{2}\right) f\left(\hat{\mathbf{I}}_{2}\right) d^{2} \hat{\mathbf{I}}_{2}-\phi\right)-\sigma\left(\hat{\mathbf{I}}_{1}\right):\left(\int_{\mathbb{S}^{2}} f\left(\hat{\mathbf{l}}_{2}\right) \frac{\frac{2}{3} \sigma\left(\hat{\mathbf{l}}_{2}\right)}{\frac{2}{3} \sigma\left(\hat{\mathbf{l}}_{2}\right): \int_{\mathbb{S}^{2}} \sigma\left(\hat{\mathbf{l}}_{3}\right) f\left(\hat{\mathbf{I}}_{3}\right) d^{2} \hat{\mathbf{I}}_{3}-\phi} d^{2} \hat{\mathbf{I}}_{2}\right)=0
$$

where $\mu$ is a Lagrange multiplier associated with the normalization of $f(\hat{\mathbf{l}})$. Solving for the distribution function gives the self-consistent equation for $f$,

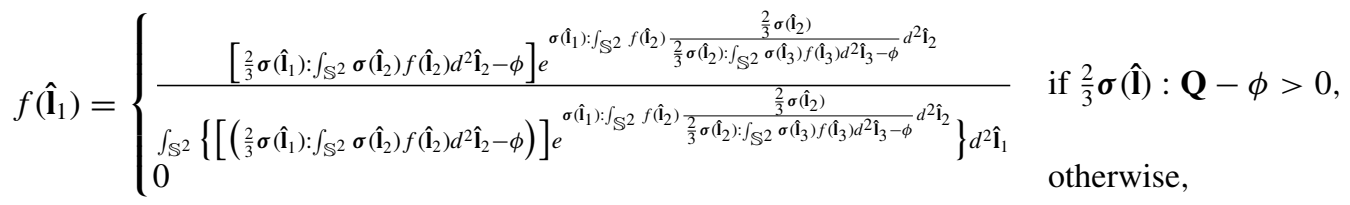

or in terms of the order parameter $\mathbf{Q}$,

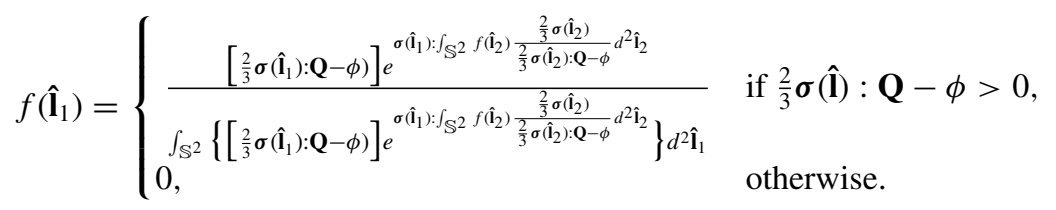

\footnotetext{
${ }^{5}$ In the mathematical literature, it is more customary to define the order tensor $\mathbf{Q}$ as the ensemble average of the orientation descriptor $\frac{2}{3} \sigma$. This makes the eigenvalues of $\mathbf{Q}$ range from $-\frac{1}{3}$ to $\frac{2}{3}$, but it leaves unchanged the definition (and the range) of the uniaxial scalar order parameter $S$ in Eq. (59).
} 
It is convenient to define the tensor

$$
\boldsymbol{\Psi}=\int_{\mathbb{S}^{2}} f(\hat{\mathbf{l}}) \frac{\frac{2}{3} \boldsymbol{\sigma}(\hat{\mathbf{I}})}{\frac{2}{3} \boldsymbol{\sigma}(\hat{\mathbf{l}}): \mathbf{Q}-\phi} d^{2} \hat{\mathbf{l}}=\left\langle\frac{\frac{2}{3} \boldsymbol{\sigma}(\hat{\mathbf{l}})}{\frac{2}{3} \boldsymbol{\sigma}(\hat{\mathbf{l}}): \mathbf{Q}-\phi}\right\rangle,
$$

which can be regarded as an auxiliary order parameter. Finally, in terms of both tensors $\mathbf{Q}$ and $\boldsymbol{\Psi}$, the expression for the distribution function becomes

$$
f(\hat{\mathbf{l}})=\frac{\left[\frac{2}{3} \boldsymbol{\sigma}(\hat{\mathbf{l}}): \mathbf{Q}-\phi\right] e^{\sigma(\hat{\mathbf{I}}): \Psi}}{\int_{\mathbb{S}^{2}}\left[\frac{2}{3} \sigma(\hat{\mathbf{l}}): \mathbf{Q}-\phi\right] e^{\sigma(\hat{\mathbf{l}}): \Psi} d^{2} \hat{\mathbf{l}}} .
$$

The requirement of positivity of the free volume fraction in expression (44) results in the orientational distribution function being zero in some regions of orientation space. There are technical issues surrounding the validity of using the EulerLagrange equation to find minimizers of singular functionals like ours, but these have been addressed in [32], rigorously providing results fully consistent with ours.

\section{The equation of state}

To derive the equation of state of our system of hard ellipsoids, we start with the relation between the pressure $P$ and the free energy density,

$$
P=-\mathcal{F}+\rho_{0} \frac{\partial \mathcal{F}}{\partial \rho_{0}} .
$$

Writing out all the terms ${ }^{6}$

$$
\begin{aligned}
\mathcal{F}= & k T \rho_{0}\left[\ln \rho_{0}+\int_{\mathbb{S}^{2}} f(\hat{\mathbf{l}}) \ln f(\hat{\mathbf{I}}) d^{2} \hat{\mathbf{l}}\right. \\
& \left.-\int_{\mathbb{S}^{2}} f(\hat{\mathbf{l}}) \ln \left(1-\rho_{0} c+\rho_{0} d \frac{2}{3} \mathbf{Q}: \boldsymbol{\sigma}(\hat{\mathbf{l}})\right) d^{2} \hat{\mathbf{l}}\right],
\end{aligned}
$$

and recalling Eq. (38) we get

$$
\begin{aligned}
& \mathcal{F}=\rho_{0} k T\left[-\ln (d / 2)+\int_{\mathbb{S}^{2}} f(\hat{\mathbf{I}}) \ln f(\hat{\mathbf{I}}) d^{2} \hat{\mathbf{I}}\right. \\
& \left.-\int_{\mathbb{S}^{2}} f(\hat{\mathbf{l}}) \ln \left(\frac{2}{3} \mathbf{Q}: \sigma(\hat{\mathbf{l}})-\phi\right) d^{2} \hat{\mathbf{l}}\right], \\
& \frac{\partial \mathcal{F}}{\partial \rho_{0}}=\rho_{0} k T\left(\int_{\mathbb{S}^{2}} \frac{f(\hat{\mathbf{l}})}{\frac{2}{3} \mathbf{Q}: \boldsymbol{\sigma}(\hat{\mathbf{I}})-\phi} d \hat{\mathbf{l}}\right) \frac{\partial \phi}{\partial \rho_{0}} \\
& +k T\left[-\ln (d / 2)+\int f(\hat{\mathbf{l}}) \ln f(\hat{\mathbf{l}}) d^{2} \hat{\mathbf{l}}\right. \\
& \left.-\int f(\hat{\mathbf{l}}) \ln \left(\frac{2}{3} \mathbf{Q}: \sigma(\hat{\mathbf{l}})-\phi\right) d^{2} \hat{\mathbf{l}}\right], \\
& \frac{\partial \phi}{\partial \rho_{0}}=\frac{1}{d \rho_{0}^{2}},
\end{aligned}
$$

${ }^{6}$ It should be noted that the term omitted in Eq. (18) would have brought an additional contribution $-k T \rho_{0}$ to the right-hand side of Eq. (51), with no consequence on the formula for $P$.

$$
\begin{aligned}
P & =\rho_{0} k T\left(\frac{1}{\rho_{0} d} \int_{\mathbb{S}^{2}} \frac{f(\hat{\mathbf{I}})}{\frac{2}{3} \mathbf{Q}: \sigma(\hat{\mathbf{l}})-\phi} d \hat{\mathbf{l}}\right) \\
& =k T \frac{1}{d}\left\langle\frac{1}{\frac{2}{3} \mathbf{Q}: \sigma(\hat{\mathbf{l}})-\phi}\right\rangle
\end{aligned}
$$

The latter can also be written as

$$
P=\rho_{0} k T\left(\frac{1}{1-\rho_{0} c}\right)\left\langle\frac{1}{1-\frac{2}{3} \phi^{-1} \mathbf{Q}: \boldsymbol{\sigma}(\hat{\mathbf{l}})}\right\rangle,
$$

which shows the distinct, and at least formally equivalent, contributions of positional and orientational entropy to the pressure.

$$
\text { If } \mathbf{Q}=\mathbf{0} \text {, then }
$$

$$
P=\frac{\rho_{0} k T}{1-\rho_{0} c},
$$

and if the ellipsoids are spheres, that is, if $\varphi=0$,

$$
P=\frac{\rho_{0} k T}{1-\rho_{0} 8 \lambda v_{0}},
$$

which coincides with the van der Waals case without attractive interactions, if we set $\lambda=\frac{1}{2}$.

In general, Eq. (56) is our equation of state. We show numerically that in the high density regime, when $\phi$ approaches 1 , the pressure approaches infinity.

\section{THE ASSUMPTION OF UNIAXIALITY}

Without external fields, classical models (such as the MaierSaupe model [33] for attractive interactions and the Onsager model [4] for steric interactions) predict only uniaxial nematic order above the ordering transition [34]. In this section, we assume that $\mathbf{Q}$ is uniaxial for simplicity. We demonstrate below that biaxial equilibrium also exists, but only as an unstable saddle point, and therefore not physically observable. Under the uniaxiality assumption, $\mathbf{Q}$ can be represented as

$$
\mathbf{Q}=\frac{S}{2}(3 \hat{\mathbf{n}} \hat{\mathbf{n}}-\mathbf{I}),
$$

where $S$ is the scalar order parameter, providing a measure of the degree of order, and $\hat{\mathbf{n}}$ is the nematic director, a unit vector indicating the direction of average orientation. One can show that then $\boldsymbol{\Psi}$ is also uniaxial and shares the same eigenframe with $\mathbf{Q}$, and thus can be written as

$$
\Psi=\Psi\left(\hat{\mathbf{n}} \hat{\mathbf{n}}-\frac{1}{3} \mathbf{I}\right)
$$

The uniaxiality assumption makes both the analysis and the numerics more tractable. Now

$$
\begin{aligned}
& \mathbf{Q}: \boldsymbol{\sigma}(\hat{\mathbf{l}})=\frac{3 S}{2} P_{2}(\hat{\mathbf{n}} \cdot \hat{\mathbf{l}}), \\
& \boldsymbol{\Psi}: \boldsymbol{\sigma}(\hat{\mathbf{l}})=\Psi P_{2}(\hat{\mathbf{n}} \cdot \hat{\mathbf{l}}),
\end{aligned}
$$


and, to within an inessential additive constant, the free energy density becomes

$$
\begin{aligned}
\mathcal{F}= & \rho_{0} k T\left(\int_{\mathbb{S}^{2}} f(\hat{\mathbf{l}}) \ln f(\hat{\mathbf{I}}) d^{2} \hat{\mathbf{I}}-\int_{\mathbb{S}^{2}} f(\hat{\mathbf{l}}) \ln \left[S P_{2}(\hat{\mathbf{n}} \cdot \hat{\mathbf{l}})-\phi\right] d^{2} \hat{\mathbf{l}}\right) \\
& =\rho_{0} k T\left(\Psi S-\ln \int_{\mathrm{S}_{+}}\left[S P_{2}(x)-\phi\right] e^{\Psi P_{2}(x)} d x\right)
\end{aligned}
$$

where $x=\hat{\mathbf{n}} \cdot \hat{\mathbf{l}}=\cos \theta, \mathrm{S}_{+}=\left\{x \in[-1,1]: S P_{2}(x)-\phi>\right.$ $0\}$, and use has been made of Eq. (49) combined with Eq. (61). The distribution function $f$, still defined (and normalized) on $\mathbb{S}^{2}$, now only depends on $x$ and is given by

$$
f(x)=\frac{1}{2 \pi} \begin{cases}\frac{\left[S P_{2}(x)-\phi\right] e^{\Psi P_{2}(x)}}{\int_{\mathrm{S}_{+}}\left[S P_{2}(x)-\phi\right] e^{\Psi P_{2}(x)} d x} & \text { if } S P_{2}(x)-\phi>0, \\ 0 & \text { otherwise. }\end{cases}
$$

Instead of solving the above self-consistent equation for $f(x)$, we solve the coupled equations for $\Psi$ and $S$,

$$
\begin{gathered}
S=\frac{\int_{\mathrm{S}_{+}} P_{2}(x)\left[S P_{2}(x)-\phi\right] e^{\Psi P_{2}(x)} d x}{\int_{\mathrm{S}_{+}}\left[S P_{2}(x)-\phi\right] e^{\Psi P_{2}(x)} d x}=\left\langle P_{2}(x)\right\rangle, \\
\Psi=\frac{\int_{\mathrm{S}_{+}} P_{2}(x) e^{\Psi P_{2}(x)} d x}{\int_{\mathrm{S}_{+}}\left[S P_{2}(x)-\phi\right] e^{\Psi P_{2}(x)} d x}=\left\langle\frac{P_{2}(x)}{S P_{2}(x)-\phi}\right\rangle .
\end{gathered}
$$

The set $S_{+}$is explicitly given by

$$
\begin{aligned}
& \mathrm{S}_{+}=\left(x_{0}, 1\right) \text { if } S>0, \\
& \mathrm{~S}_{+}=\left(0, x_{0}\right) \text { if } S<0,
\end{aligned}
$$

where $x_{0}$ is the positive root of $^{7}$

$$
S P_{2}\left(x_{0}\right)-\phi=0 .
$$

To solve the above equations efficiently, by use of Eq. (69), we eliminate $\phi$ from them, thus arriving at the following equations:

$$
\begin{gathered}
S=\frac{\int_{\mathrm{S}_{+}} P_{2}(x)\left[P_{2}(x)-P_{2}\left(x_{0}\right)\right] e^{\Psi P_{2}(x)} d x}{\int_{\mathrm{S}_{+}}\left[P_{2}(x)-P_{2}\left(x_{0}\right)\right] e^{\Psi P_{2}(x)} d x}, \\
\frac{1}{\Psi}=\frac{\int_{\mathrm{S}_{+}} P_{2}^{2}(x) e^{\Psi P_{2}(x)} d x}{\int_{\mathrm{S}_{+}} P_{2}(x) e^{\Psi P_{2}(x)} d x}-P_{2}\left(x_{0}\right) .
\end{gathered}
$$

The latter equation is solved for $x_{0}$, separately for the two cases in Eqs. (67) and (68). For each of these cases, we first assign a value for $\Psi \in(-\infty, \infty)$, then $x_{0}$ is found by using the built-in root finding routine in Wolfram MATHEMATICA. Next, the order parameter $S$ can be evaluated from Eq. (70). Finally $\phi$ can be obtained from Eq. (69). In this way, we associate each value of $\Psi$ with the scalar order parameter $S$ and the parameter $\phi$ expressing the excluded volume fraction.

\footnotetext{
${ }^{7}$ We study $f$ only for $0 \leqslant x \leqslant 1$, as by Eq. (64) it is even in $-1 \leqslant$ $x \leqslant 1$.
}

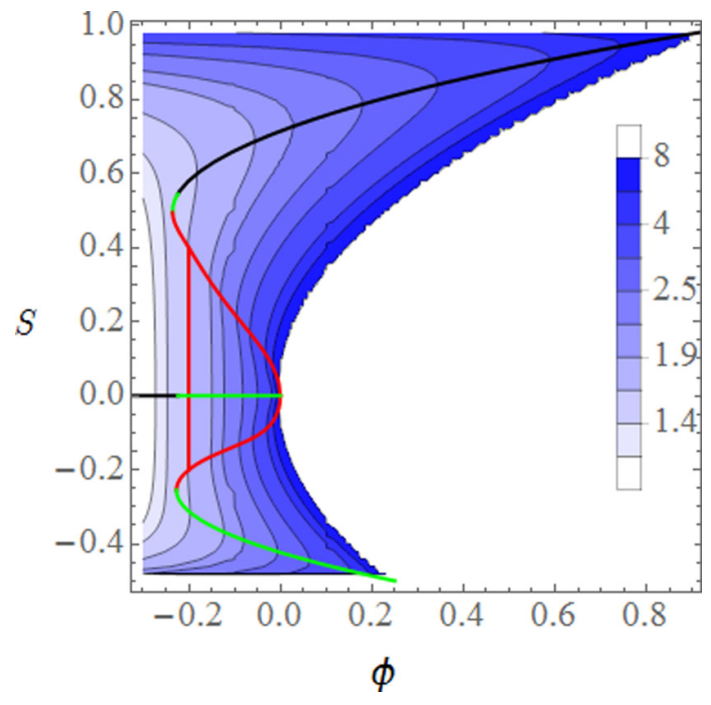

FIG. 1. Equilibrium order parameter $S$ versus $\phi$ with uniaxial solutions only, superimposed on the energy per particle contours. The black curve represents the stable branch of the bifurcation, the green (light gray) curves correspond to the metastable branches, and the red (dark gray) curves to the unstable branches.

\section{A. A special point}

The point $\Psi=0$ is a singular point of the above equations. In this case, we consider Eq. (66) directly. It is easy to show that the integration limit $x_{0}=0$ if $S>0$, whereas $x_{0}=1$ if $S<0$. By setting $\Psi=0$ in Eq. (65), we get $\phi=-0.2$. To satisfy the constraint

$$
S P_{2}(x)-\phi>0,
$$

we immediately arrive at the following range for $S$ :

$$
-0.2<S<0.4 \text {. }
$$

That is, for $\Psi=0$, corresponding to $\phi=-0.2$, any value of $S \in(-0.2,0.4)$ is a solution of the Euler-Lagrange equation.

\section{B. Homogeneous equilibrium solution}

Figure 1 illustrates our key result. It shows the equilibrium uniaxial order parameter $S$ versus $\phi$. The stability of different branches is determined by examining the local convexity of the free energy density. To effectively illustrate this, the contours of free energy per particle are superimposed on the bifurcation graph in the $S-\phi$ plane. The black curve represents the stable branch. The green (light gray) curves correspond to metastable states, and the red (dark gray) curves indicate unstable regimes. For $\phi<\phi_{\mathrm{NI}}=-0.224$, the system is in the isotropic state with $S=0$; at $\phi=\phi_{\mathrm{NI}}$, the system undergoes a first order transition to the nematic state, with order parameter $S_{\mathrm{NI}}=$ 0.545 . As $\phi \rightarrow 1$, the order parameter $S \rightarrow 1$ indicating a completely aligned configuration. Inside the blue parabola are the regions where the order parameters of the ordered state are not admissible. At $\phi=-0.2$, the vertical red (dark gray) line indicates that all values of $-0.2<S<0.4$ share the same energy.

In Fig. 2, representative orientational probability density functions are presented as a density plot on the surface of a 


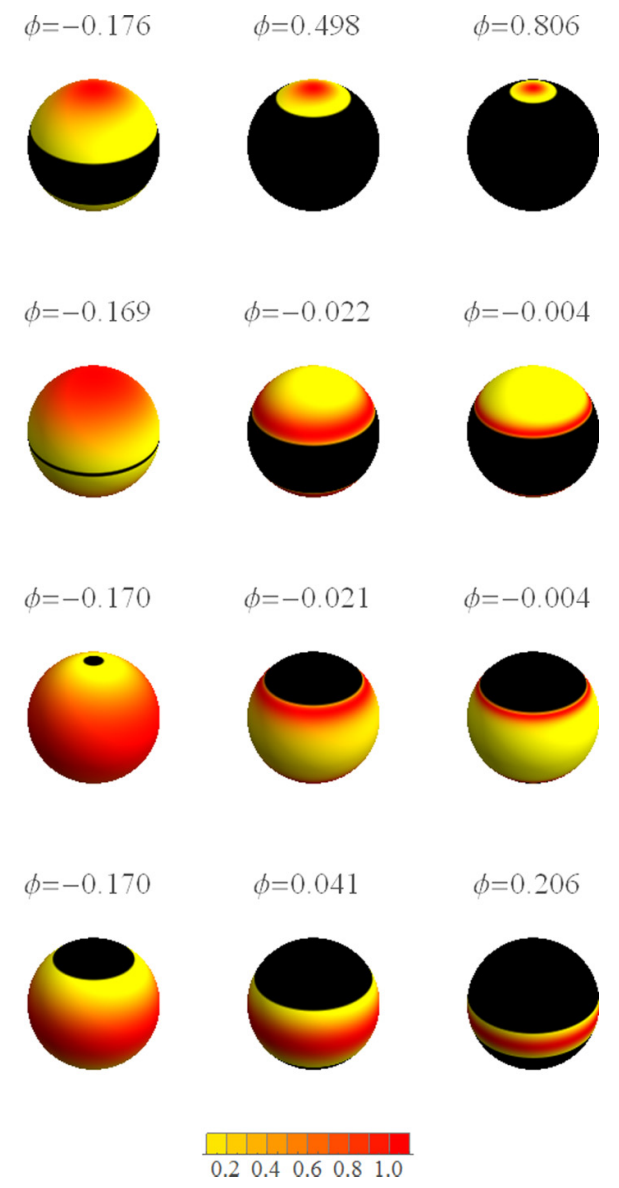

FIG. 2. Density plot of the orientational distribution function for selected equilibrium uniaxial solutions. Top row, stable configuration with order parameter $S>0$; second row, unstable configuration with $S>0$; third row, unstable saddle point configuration with $S<0$; bottom row, metastable configuration with $S<0$.

unit sphere for different equilibrium uniaxial solutions shown in Fig. 1. In those graphs, the $z$ axis is chosen to be along the uniaxial director; thus the density plots are axisymmetric with respect to the $z$ axis. The density functions are continuous, and normalized by their maximum values on each sphere. The black regions on different spheres indicate forbidden orientations; i.e., no particles are allowed to orient in directions corresponding to those regions. Yellow (light gray) indicates that only relatively few particles are oriented in that direction, and red (dark gray) indicates that the majority of particles are oriented in that direction. The figures clearly reveal that the allowed regions of orientation shrink as the number density increases. This effect is further illustrated in Fig. 3.

Figure 3 shows the lower (upper) limit $x_{0}$ of the integration for $S>0(S<0)$. The probability distribution function has no forbidden region when $\Psi=0$. As one traces the stable black branch [Fig. 3(a)] in the direction of increasing $\phi$, the region shrinks. As $\phi \rightarrow 1$, the lower limit $x_{0} \rightarrow 1$, which implies that all particles orient precisely in the same direction; that is, they are perfectly ordered. At the lowest green (light gray) branch [Fig. 3(b)], as $\phi \rightarrow 1 / 4$, the upper limit $x_{0} \rightarrow 0$, and the particles all lie in the plane perpendicular to the unique direction (normal to the plane) but are randomly oriented in

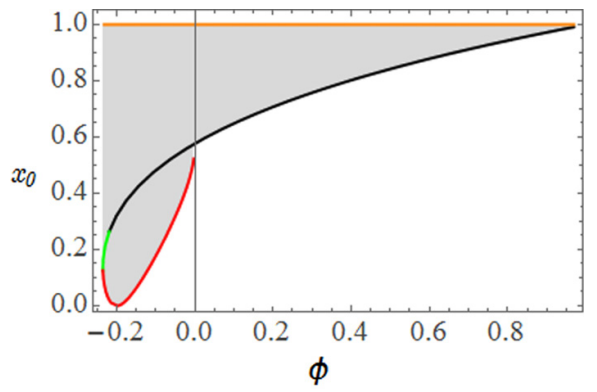

(a)

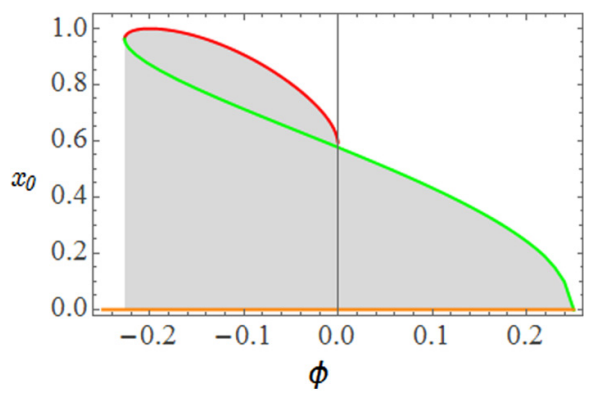

(b)

FIG. 3. (a) Lower integration limit $x_{0}$ versus $\phi$ for uniaxial equilibrium solutions with $S>0$; the top horizontal line indicates the upper limit of the integration. (b) Upper limit $x_{0}$ vs $\phi$ for equilibrium solutions with $S<0$; the bottom horizontal line indicates the lower limit of the integration. Black, green (light gray), and red (dark gray) colors correspond to stable, metastable, and unstable solutions in Fig. 1.

that plane. On the unstable red (dark gray) branches [Figs. 3(a) and $3(\mathrm{~b})$ ], the particles are oriented inside or outside a cone, but more towards the boundary of that cone, as shown in the second and third rows in Fig. 2.

\section{Phase coexistence}

For completeness, we next briefly inquire about the possibility of coexisting nematic and isotropic phases in regions of $\phi$ where both isotropic and nematic solutions exist. We ask therefore whether the total free energy of the system can be reduced by phase separation. Rather than plotting the free energy density versus the number density, as is customary, In Fig. 4 we plot the free energy per particle $\mathcal{F} / \rho_{0}$ versus $\phi$, and implement the classical double tangent construction. Here the common slope indicates equal pressures, and the linear dependence indicates equal chemical potentials of the two coexisting phases. Using this representation, we learn that the nematic and isotropic phases coexist with the universal dimensionless parameter values $\phi_{\mathrm{I}}=-0.3652$ and $\phi_{\mathrm{N}}=$ 0.1634 , regardless of particle aspect ratio. The phase transition for the homogeneous nematic phase occurs at $\phi_{\mathrm{NI}}=-0.224$.

The volume fractions $\eta=\rho_{0} v_{0}$ of the two coexisting phases as function of the aspect ratio $\kappa$ can be obtained at once from the values of $\phi_{\mathrm{I}}$, and $\phi_{\mathrm{N}}$ though Eq. (38), once $\lambda$ is assigned. 


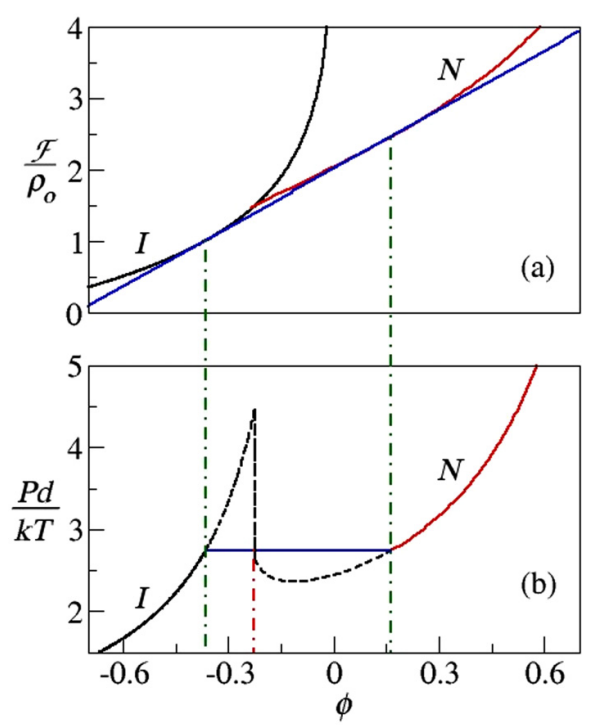

FIG. 4. These diagrams illustrate two-phase coexistence: (a) the double tangent construction and (b) the Maxwell, equal area construction. The dependence of pressure on $\phi$ in the isotropic and nematic phases is also shown in (b).

\section{BIAXIAL EQUILIBRIUM SOLUTIONS}

We anticipate the existence of biaxial equilibrium solutions, especially for $\phi>0$, since the energy graph for each $\phi>0$ corresponds to two separated branches, with inadmissible regions in between. The energy diverges as $S$ gets near the boundary of the inadmissible region as seen in Fig. 1. If the system starts with a configuration with $S<0$ near the metastable phase, it has to make its way to the lowest energy state, and the only way is through biaxial phases. In its eigenframe, $\mathbf{Q}$ can be represented as

$$
\mathbf{Q}=\operatorname{diag}\left[r \cos \left(\alpha+\frac{2 \pi}{3}\right), r \cos \left(\alpha-\frac{2 \pi}{3}\right), r \cos \alpha\right],
$$

thus $\mathbf{Q}$ is fully characterized by the parameters $r$ and $\alpha$ [35]. We use a ternary diagram to represent $\mathbf{Q}$. Consider an equilateral triangle with sides of unit length, centered on the origin, with one vertex on the positive $y$ axis. Each point in the triangle corresponds to a set of eigenvalues of $\mathbf{Q}$. To obtain the eigenvalues of $\mathbf{Q}$, we draw lines parallel to each edge, through the point. Each line intersects two edges, and the length of the line segment from the point of intersection to the vertex gives the eigenvalues of $\mathbf{Q}+\frac{1}{3} \mathbf{I}$. We illustrate this in Fig. 5 . Due to symmetry, we only consider the shaded $1 / 6$ portion of the triangle; the rest corresponds to five different permutations of the same sets of eigenvalues of $\mathbf{Q}$. Given two coordinates $(x, y), 0<x<\sqrt{3} / 2,-1 / 2<y<\sqrt{3} x / 3$, of a point, $r$ and $\alpha$ can be obtained from

$$
\begin{gathered}
r=\sqrt{x^{2}+y^{2}}=\sqrt{\frac{2}{3} \mathbf{Q}: \mathbf{Q}}, \\
\alpha=-\frac{\pi}{6}-\arctan \frac{y}{x} .
\end{gathered}
$$

Here $r=0$, for which $\mathbf{Q}=\operatorname{diag}(0,0,0)$, corresponds to the isotropic phase (the upper left corner of the shaded triangle in Fig. 5); $\alpha=0$, for which $\mathbf{Q}=\operatorname{diag}(-r / 2,-r / 2, r)$, corresponds to uniaxial prolate phases (the hypotenuse), and $\alpha=\pi / 3$, for which $\mathbf{Q}=\operatorname{diag}(-r, r / 2, r / 2)$, corresponds to uniaxial oblate phases (the short vertical edge); $\alpha=\pi / 6$, for which $\mathbf{Q}=\operatorname{diag}(-\sqrt{3} r / 2,0, \sqrt{3} r / 2)$, correspond to biaxial phase with largest biaxiality.

We select two representative values of $\phi$ to examine the landscape of the free energy density versus $\mathbf{Q}$. For each value of $\phi$, we specify $\mathbf{Q}$, and numerically solve for $\Psi$ using an iterative Newton method, and then numerically evaluate the energy density. In Fig. 6, energy density contours are plotted versus different $\mathbf{Q}$. For $\phi=-0.1$, there are six critical points on the energy surface: one isotropic local minimizer, one prolate uniaxial global minimizer, one uniaxial oblate local maximizer along the hypotenuse, one uniaxial oblate local minimizer, one uniaxial oblate saddle point along the short vertical edge, and one biaxial saddle point in the interior of the triangle. For $\phi=0.1$, there are three critical points: one uniaxial prolate local minimizer along the hypotenuse, one uniaxial oblate local minimizer along the short vertical edge, and one biaxial saddle point in the interior of the triangle. The energy blows up when $r^{2}<\phi$, so there is an inadmissible region in the upper left portion of the triangle. In the regime where $\phi>0$, and the $S<0$ equilibrium solution is stable to biaxial perturbations, we expect a saddle point corresponding to a "mountain pass" between the $S>0$ and $S<0$ local minimizers. As we have demonstrated there are no other uniaxial critical points in this regime, this saddle point must be biaxial. Such behavior was not reported in Onsager's work, nor, as far as we know, in any model for uniaxial particles without an external field.

\section{COMPARISON WITH SIMULATIONS}

Since the early times of Frenkel and Mulder's simulations with rotationally symmetric ellipsoids [2], this system of hard particles has become the test case for all theories that have attempted to explain the behavior of dense nematics; we could not elude such a test.

Much is known now about the ordering transitions that take place in this system as the number density is increased, including exotic crystal phases, such as the newly discovered SM2 phase [36-38], which supplements the stretched fcc structure already known from [2]. New points on the isotropicto-nematic transition line of the phase diagram have also been added for larger values of the aspect ratio $\kappa$ in $[39,40]$. This latter also presents a full account on all the phases presently known, enriching with new details the bell-shaped diagram already hypothesized in [2] (see, in particular, Fig. 5 of [40]). Such a diagram has also proven to be relevant to dynamical studies $[41,42]$, as its shape is reflected by the rotational isodiffusivity lines in the $\left(\kappa^{-1}, \eta\right)$ plane, where $\eta=\rho_{0} v_{0}$ is the volume fraction. It was shown in these studies how the isotropic-to-nematic transition is heralded by a progressive hampering of the rotational dynamics, which would lead particles to a complete arrest of the rotational motion, were this not preempted by the ordering nematic transition.

Clearly, neither the crystal phases nor the dynamical precursors of the nematic phase are within the reach of our 


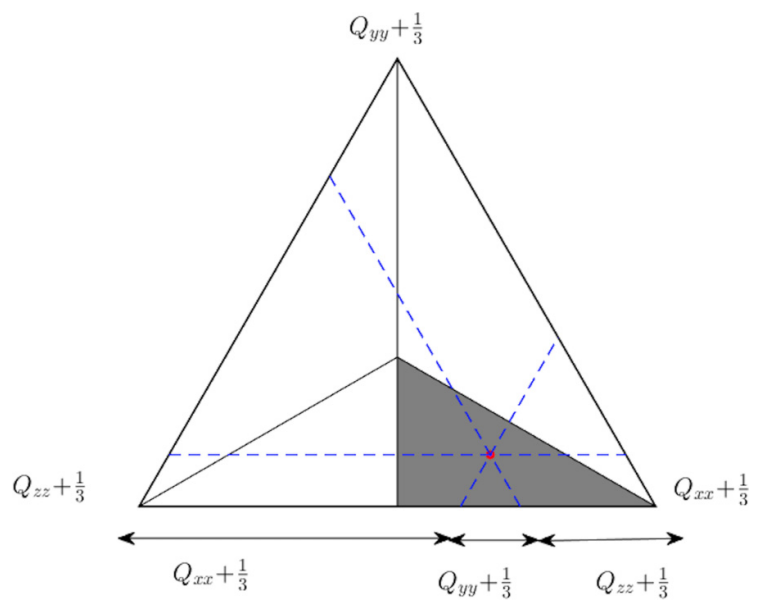

FIG. 5. Ternary diagram for representing $\mathbf{Q}$.

theory. But we can still contrast the latter with the simulation data available for the transition value $\eta_{\mathrm{NI}}$ of $\eta$.

It readily follows from Eqs. (38), (32), and (31) that our theory predicts the following dependence for $\eta_{\mathrm{NI}}$ on the particles' anisotropy:

$$
\eta_{\mathrm{NI}}=\frac{1}{8 \lambda} \frac{1}{1+\left(1-\phi_{\mathrm{NI}}\right) \varphi(\epsilon)},
$$

where $\varphi(\epsilon)$ is the shape function expressed explicitly by Eq. (A7) in terms of the particles' eccentricity defined in Eq. (33). Letting $\phi_{\mathrm{NI}}=-0.244$, as discussed above, we can use Eq. (77) to determine $\lambda$ so as to fit the data available from simulations for the isotropic-to-nematic transition. The comparison of the graph of $\eta_{\mathrm{NI}}$ with 41 data points taken from [2], [39], and [40] is shown in Fig. 7.

Since $\eta_{\mathrm{NI}}$ depends only on the eccentricity $\epsilon$ of the ellipsoids, we have collected data for both prolate and oblate ellipsoids and plotted them for the effective aspect ratio $\kappa>1$ (which is the real aspect ratio for prolate ellipsoids and its reciprocal for oblate ellipsoids). The best least-squares fit is found for

$$
\frac{1}{\lambda} \doteq 6.065
$$

which is close to the value for close packed spheres in Eq. (15). Setting $\frac{1}{\lambda}=6$ turns Eq. (77) into our explicit, theoretical

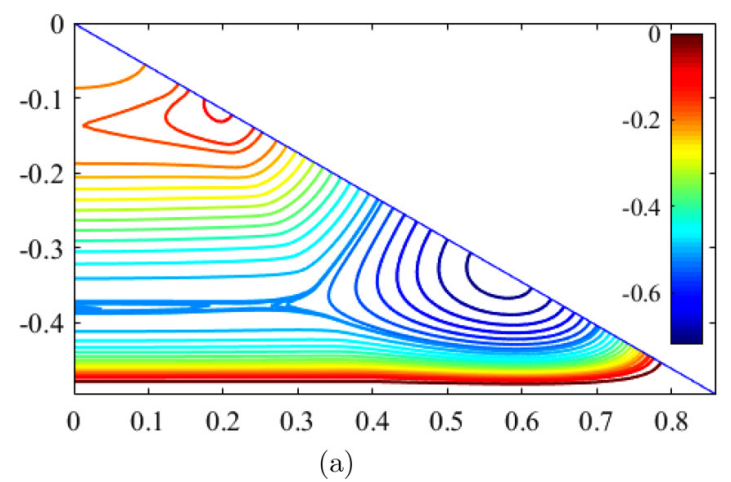

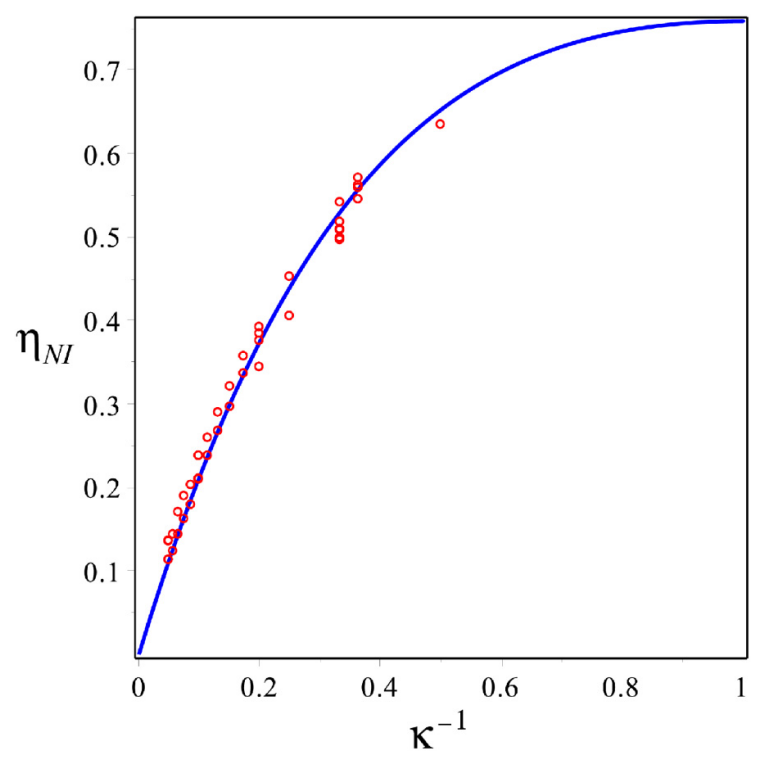

FIG. 7. The graph of $\eta_{\mathrm{NI}}$ as in Eq. (77) against the reciprocal $\kappa^{-1}$ of the aspect ratio for $\lambda$ as in Eq. (78). Simulation data are represented by red circles. Data have been collected from [2] and [40] for both prolate and oblate ellipsoids, and reported here for the effective aspect ratio $\kappa>1$ (which is the real aspect ratio for prolate ellipsoids and its reciprocal for oblate ellipsoids). Data taken from [2] (see the first two columns of their Table 7) and from [39] (see the fifth and eighth columns of their Table VI, where $\rho_{\mathrm{cp}}=\sqrt{2}$ ) include both ends of the coexistence range (and their $\eta$ values have been multiplied by $\pi / 6$ to account for the fact that the data of [2] and [39] are scaled to the volume $\left.L W^{2}=6 v_{0} / \pi\right)$. Data taken from [40] are all those in the first column of Table I, but the two referring to the cases 1.3:1-prolate and 1:1.3-oblate, as these latter fall inside the crystal region of the phase diagram (as also shown in Fig. 5 of [40]).

prediction for the isotropic-to-nematic transition line for a fluid of hard ellipsoids of revolution.

\section{CONCLUSIONS}

In this paper, we proposed a theory for dense nematics whose constituting molecules interact only through the entropic forces arising from mutual interpenetration. Our emphasis was on the behavior at high densities and the

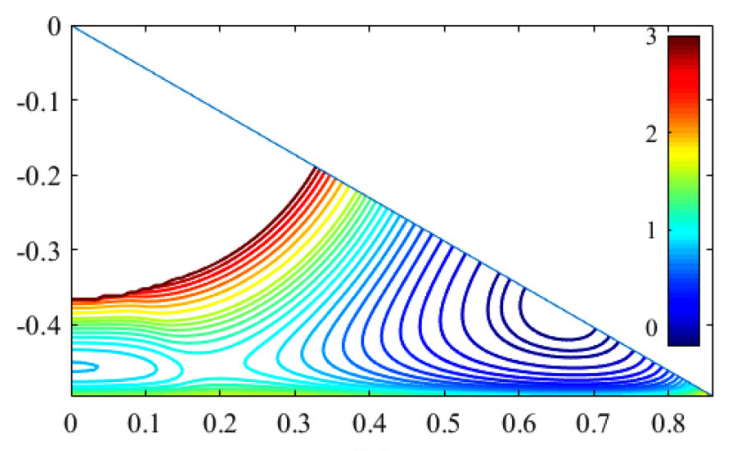

(b)

FIG. 6. Energy contours for two representative volume fractions: (a) $\phi=-0.1$ and (b) $\phi=0.1$. 
effects of the depletion of available orientational states on orientational order.

We studied a system of hard ellipsoidal particles and compared the predictions of our theory with simulation data on the isotropic-to-nematic transition, finding a good agreement for a specific value of a single fitting parameter $\lambda$. This parameter should more generally be a function of the number density. Work to determine the dependence of $\lambda$ on density is currently under way.

Our study was phrased in the canonical ensemble; we derived an expression for the free energy at the van der Waals level. Low density expansions of the logarithmic term agree with the free energy of Onsager [4] and Doi and Edwards [29], p. 354. A major challenge was the determination of the orientational distribution function subject to the hard constraint that the number states available to the system be positive. One striking result of this constraint, in the mean field limit, is that at densities above the isotropic-to-nematic transition, the orientational distribution function which minimizes the free energy has a compact support over orientation space. Although strictly forbidden orientations are likely an artifact of our mean field approach, where particles effectively behave identically, we expect that more sophisticated models would similarly show a strong suppression of the corresponding orientational states.

We have found that the quantity $\phi$, related to the orientational relative free volume, is a convenient control parameter characterizing the state of the system, regardless of density or particle aspect ratio. Our model predicted a first order nematic-isotropic phase transition; in a uniform system, the NI transition occurs at $\phi_{\mathrm{NI}}=-0.224$. In the regime $-0.3652 \leqslant$ $\phi \leqslant 0.1634$, the system is unstable and undergoes phase separation. As $\phi$ and the density increase, the regions of forbidden orientations grow, and the degree of orientational order increases; the system becomes perfectly aligned in the dense packing limit.

The equation of state from our free energy expression is more realistic than Onsager's in that in our model, the pressure diverges in the dense packing limit; our equation of state shows the individual contributions of positional and orientational entropy to the pressure.

We see this work as providing a starting point towards a more systematic study of dense nematic systems. Future work is aimed at identifying on a first-principles basis the function of density that is to replace the single parameter $\lambda$ that here ensured agreement between theory and simulation data.

\section{ACKNOWLEDGMENTS}

X.Z. and P.P.-M. acknowledge support from NSF under Grants No. DMS-1212046 and No. EFRI-1332271. E.S.N. acknowledges support under FAPESP Grant No. 2016/074485. J.M.T. acknowledges support from the European Research Council under the European Union's Seventh Framework Programme (FP7/2007-2013)/ERC Grant Agreement No. 291053. E.G.V. acknowledges the kind hospitality of the Oxford Centre for Nonlinear PDE, where part of this work was done while he was visiting the Mathematical Institute at the University of Oxford.

\section{APPENDIX: EXCLUDED VOLUME OF ELLIPSOIDS OF REVOLUTION}

In this Appendix we justify the approximate form for the excluded volume of two congruent ellipsoids of revolution adopted in this paper and we compare it with other approximations used in the literature.

Symmetry demands that the excluded volume of two congruent bodies of revolution be a function $V_{\mathrm{exc}}(x)$ of the inner product $x=\hat{\mathbf{l}}_{1} \cdot \hat{\mathbf{l}}_{2}=\cos \theta$ between the unit vectors designating their axes. In general, $V_{\text {exc }}$ can be expanded in a series of Legendre polynomials,

$$
V_{\mathrm{exc}}(x)=\sum_{k=0}^{\infty} B_{k} P_{k}(x) .
$$

It was shown in [43] that for convex particles all $B_{k}$ can be expressed in terms of a countable family of extended invariant Minkowski shape functionals, which can be computed explicitly for special shapes such as circular cones and ellipsoids of revolution. The formula for $B_{0}$, which represents the isotropic average of $V_{\text {exc }}$, had been known to be a function of the classical invariant Minkowski functionals at least since the work of Isihara [44]. It is easy to show that all odd-indexed coefficients $B_{k}$ vanish identically for particles that lack shape polarity.

For an ellipsoid of revolution with eccentricity $\epsilon$,

$$
\begin{gathered}
B_{0}=\frac{3}{2} v_{0}\left[\frac{4}{3}+\left(1+\left(1-\epsilon^{2}\right) \frac{\operatorname{arctanh} \epsilon}{\epsilon}\right)\left(1+\frac{\arcsin \epsilon}{\epsilon \sqrt{1-\epsilon^{2}}}\right)\right], \\
B_{2}=\frac{15}{32} v_{0} \frac{1}{\epsilon^{4}}\left(\epsilon^{2}-3+\left(\epsilon^{2}+3\right)\left(1-\epsilon^{2}\right) \frac{\operatorname{arctanh} \epsilon}{\epsilon}\right) \\
\times\left(3-2 \epsilon^{2}+\frac{4 \epsilon^{2}-3}{\epsilon \sqrt{1-\epsilon^{2}}} \arcsin \epsilon\right),
\end{gathered}
$$

where $v_{0}$ is the particle's volume. The former formula was already given in [44], while the latter formula coincides with that given in [45] only for oblate ellipsoids (as pointed out in [43], where Eq. (A3) was established for all ellipsoids of revolution, the formula of [45] for prolate ellipsoids fails to be invariant under the transformation $\kappa \mapsto 1 / \kappa$, and it is thus bound to be incorrect).

We wish to approximate $V_{\text {exc }}(x)$ with

$$
V_{\mathrm{exc}}^{(\mathrm{app})}(x)=C-D P_{2}(x),
$$

with $C$ and $D$ constants chosen in such a way that

$$
V_{\mathrm{exc}}^{(\mathrm{app})}(1)=8 v_{0}
$$

and

$$
V_{\mathrm{exc}}^{(\mathrm{app})}(0)=B_{0}+B_{2} P_{2}(0)=B_{0}-\frac{1}{2} B_{2},
$$

where $B_{0}$ and $B_{2}$ are as in Eqs. (A2) and (A3). It is a simple matter to show that $C$ and $D$ can be written as in Eqs. (31) and (32) with the shape function $\varphi$ defined as

$$
\begin{aligned}
\varphi(\epsilon)= & \frac{1}{768} \frac{1}{\epsilon^{6}}\left\{254 \epsilon^{6}-135 \epsilon^{4}+135 \epsilon^{2}\right. \\
& +9 \epsilon\left(4 \epsilon^{4}+25 \epsilon^{2}-15\right) \frac{\arcsin \epsilon}{\sqrt{1-\epsilon^{2}}}
\end{aligned}
$$




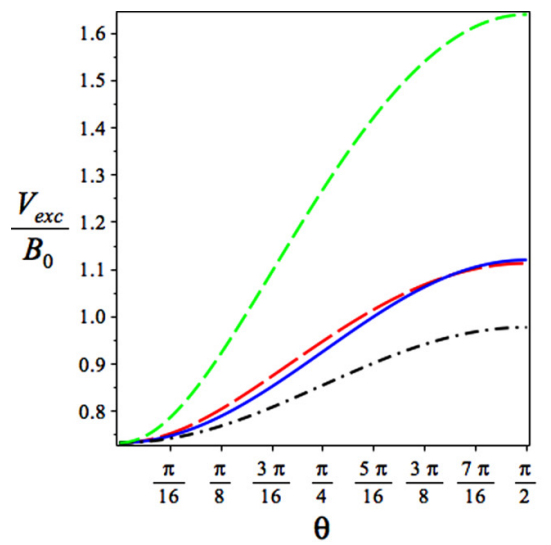

(a)

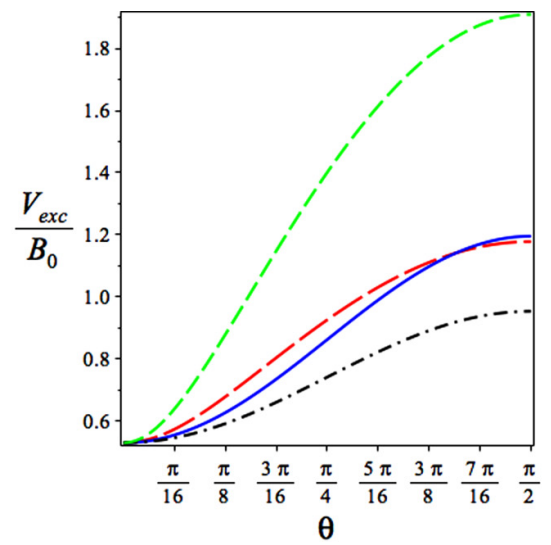

(b)

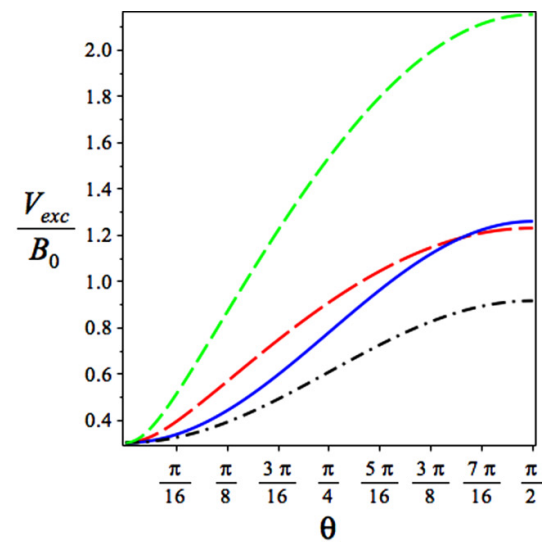

(c)

FIG. 8. The excluded volume of a pair of congruent ellipsoids of revolution $V_{\text {exc }}$ (normalized to its isotropic average $B_{0}$ ) is plotted against the angle $\theta$ between their symmetry axes according to four different approximate formulas: (long-dashed red lines) the expansion in Eq. (A1) truncated at $k=20$ with the coefficients computed according to the theory of [43]; (solid blue lines) our approximate formula in Eq. (A4); (dash-dotted black lines) Sheng's formula from [21]; and (dashed green lines) the HGO formula in Eq. (A11). Values of the ellipsoids' aspect ratio: (a) $\kappa=3$, (b) $\kappa=5$, and (c) $\kappa=10$.

$$
\begin{aligned}
& +9 \epsilon\left(1-\epsilon^{2}\right)\left(14 \epsilon^{4}+5 \epsilon^{2}-15\right) \operatorname{arctanh} \epsilon \\
& \left.+9\left(1-\epsilon^{2}\right)\left(4 \epsilon^{4}-15 \epsilon^{2}+15\right) \frac{\arcsin \epsilon}{\sqrt{1-\epsilon^{2}}} \operatorname{arctanh} \epsilon\right\}-\frac{2}{3}
\end{aligned}
$$

In the main text we have adopted the function in Eq. (A4) [and dropped the cumbersome superscript (app)]. The function $\varphi$ is positive and monotonically increasing on the interval $[0,1)$; it diverges as $\epsilon \rightarrow 1^{-}$and possesses the following asymptotic behaviors:

$$
\begin{gathered}
\lim _{\epsilon \rightarrow 0} \epsilon^{-4} \varphi(\epsilon)=\frac{1}{15}, \\
\lim _{\epsilon \rightarrow 1^{-}} \sqrt{1-\epsilon^{2}} \varphi(\epsilon)=\frac{21 \pi}{256} .
\end{gathered}
$$

Other approximate formulas have been proposed in the past for the excluded volume of ellipsoids of revolution. Sheng [21] used a formula like the one in Eq. (A4), for which $\varphi$ would be replaced by the following simpler form:

$$
\varphi(\epsilon)=\frac{\left(\sqrt{1-\epsilon^{2}}-1\right)^{2}}{\sqrt{1-\epsilon^{2}}} .
$$

Berne and Penchukas [46] introduced the hard Gaussian overlap model (customarily abbreviated HGO in the literature), which mimics a short range repulsion between elongated molecules (see [47] for a lucid description of this model and its connections with traditional hardparticle models). When applied to hard ellipsoids, this model delivers an effective excluded volume written in the form

$$
V_{\mathrm{exc}}^{(\mathrm{HGO})}(x)=8 v_{0} \sqrt{\frac{1-\chi^{2} x^{2}}{1-\chi^{2}}}, \quad \text { where } \quad \chi^{2}=\frac{\kappa^{2}-1}{\kappa^{2}+1} .
$$

It is instructive to compare our approximate formula in Eq. (A4), Sheng's variant, and the HGO formula with the remarkably good (at least for $\kappa \leqslant 20$ ) but highly inconvenient expression obtained from Eq. (A1) by truncating the series expansion at $k=20$ and computing its coefficients exactly through the theory presented in [43]. Figure 8 illustrates such a comparison for $\kappa=3,5$, and 10. While the HGO formula highly overestimates the excluded volume, Sheng's formula underestimates it (though less dramatically so). By contrast, the formula we have used in this study seems to be more faithful, at least to the truncated Legendre expansion.
[1] D. Frenkel, J. P. McTague, and B. M. Mulder, Phase Diagram of a System of Hard Ellipsoids, Phys. Rev. Lett. 52, 287 (1984).

[2] D. Frenkel and B. M. Mulder, The hard ellipsoid-of-revolution fluid: I. Monte Carlo simulations, Mol. Phys. 55, 1171 (1985).

[3] B. M. Mulder and D. Frenkel, The hard ellipsoid-of-revolution fluid: II. The $y$-expansion equation of state, Mol. Phys. 55, 1193 (1985).

[4] L. Onsager, The effects of shape on the interaction of colloidal particles, Ann. N. Y. Acad. Sci. 51, 627 (1949).
[5] P. Palffy-Muhoray, E. G. Virga, and X. Zheng, Onsager's missing steps retraced, arXiv:1707.07761.

[6] L. Mederos, E. Velasco, and Y. Martinez-Raton, Hard-body models of bulk liquid crystals, J. Phys.: Condens. Matter 26, 463101 (2014).

[7] J. E. Mayer and M. G. Mayer, Statistical Mechanics (Wiley, New York, 1940).

[8] J. P. Straley, The gas of long rods as a model for lyotropic liquid crystals, Mol. Cryst. Liq. Cryst. 22, 333 (1973). 
[9] J. P. Straley, Third virial coefficient for the gas of long rods, Mol. Cryst. Liq. Cryst. 24, 7 (1973).

[10] B. Tjipto-Margo and G. T. Evans, The Onsager theory of the isotropic-nematic liquid crystal transition: Incorporation of the higher virial coefficients, J. Chem. Phys. 93, 4254 (1990).

[11] B. Barboy and W. M. Gelbart, Series representation of the equation of state for hard particle fluids, J. Chem. Phys. 71, 3053 (1979).

[12] B. Barboy and W. M. Gelbart, Hard-particle fluids. II. Generalyexpansion-like descriptions, J. Stat. Phys. 22, 709 (1980).

[13] R. Zwanzig, First-order phase transition in a gas of long thin rods, J. Chem. Phys. 39, 1714 (1963).

[14] J. D. Parsons, Nematic ordering in a system of rods, Phys. Rev. A 19, 1225 (1979).

[15] S.-D. Lee, A numerical investigation of nematic ordering based on a simple hard-rod model, J. Chem. Phys. 87, 4972 (1987).

[16] N. F. Carnahan and K. E. Starling, Equation of state for nonattracting rigid spheres, J. Chem. Phys. 51, 635 (1969).

[17] S.-D. Lee, The Onsager-type theory for nematic ordering of finite-length hard ellipsoids, J. Chem. Phys. 89, 7036 (1988).

[18] M. Baus, J.-L. Colot, X.-G. Wu, and H. Xu, Finite-Density Onsager-Type Theory for the Isotropic-Nematic Transition of Hard Ellipsoids, Phys. Rev. Lett. 59, 2184 (1987).

[19] J.-L. Colot, X.-G. Wu, H. Xu, and M. Baus, Density-functional, Landau and Onsager theories of the isotropic-nematic transition of hard ellipsoids, Phys. Rev. A 38, 2022 (1988).

[20] P. Palffy-Muhoray and B. Bergersen, Van der Waals theory for nematic liquid crystals, Phys. Rev. A 35, 2704 (1987).

[21] P. Sheng, Hard rod model of the nematic-isotropic phase transition, in Introduction to Liquid Crystals, edited by E. B. Priestley, P. J. Wojtowicz, and P. Sheng (Plenum Press, Boston, 1974), pp. 59-70.

[22] M. P. Taylor and J. Herzfeld, Nematic and smectic order in a fluid of biaxial hard particles, Phys. Rev. A 44, 3742 (1991).

[23] A. Malijevsky, G. Jackson, and S. Varga, Many-fluid Onsager density functional theories for orientational ordering in mixtures of anisotropic hard-body fluids, J. Chem. Phys. 129, 144504 (2008).

[24] L. Wu, A. Malijevsky, G. Jackson, E. A. Muller, and C. Avendano, Orientational ordering and phase behaviour of binary mixtures of hard spheres and hard spherocylinders, J. Chem. Phys. 143, 044906 (2015).

[25] J. N. Israelachvili, Intermolecular and Surface Forces (Academic Press, Waltham, MA, 1992).

[26] E. C. Gartland, Jr., and E. G. Virga, Minimum principle for indefinite mean-field free energies, Arch. Ration. Mech. Anal. 196, 143 (2010).

[27] P. Palffy-Muhoray, The single particle potential in mean-field theory, Am. J. Phys. 70, 433 (2002).
[28] A. M. Sonnet and E. G. Virga, Dissipative Ordered Fluids: Theories for Liquid Crystals (Springer, New York, 2012).

[29] M. Doi and S. F. Edwards, The Theory of Polymer Dynamics (Clarendon Press, Oxford, U.K., 1988).

[30] N. G. van Kampen, Condensation of a classical gas with long range attraction, Phys. Rev. 135, A362 (1964).

[31] L. Tonks, The complete equation of state of one, two and threedimensional gases of hard elastic spheres, Phys. Rev. 50, 955 (1936).

[32] J. M. Taylor, An analysis of equilibria in dense nematic liquid crystals, arXiv: 1702.08793.

[33] W. Maier and A. Saupe, Eine einfache molekulare Theorie des nematischen kristallinflüssigen Zustandes, Z. Naturforsch. 13a, 564 (1958)

[34] I. Fatkullin and V. Slastikov, Critical points of the Onsager functional on a sphere, Nonlinearity 18, 2565 (2005).

[35] X. Zheng and P. Palffy-Muhoray, One order parameter tensor mean field theory for biaxial liquid crystals, Discrete Contin. Dyn. Syst. B 15, 475 (2011).

[36] P. Pfleiderer and T. Schilling, Simple monoclinic crystal phase in suspensions of hard ellipsoids, Phys. Rev. E 75, 020402(R) (2007).

[37] A. Donev, F. H. Stillinger, P. M. Chaikin, and S. Torquato, Unusually Dense Crystal Packings of Ellipsoids, Phys. Rev. Lett. 92, 255506 (2004).

[38] M. Radu, P. Pfleiderer, and T. Schilling, Solid-solid phase transition in hard ellipsoids, J. Chem. Phys. 131, 164513 (2009).

[39] P. J. Camp, C. P. Mason, M. P. Allen, A. A. Khare, and D. A. Kofke, The isotropic-nematic phase transition in uniaxial hard ellipsoid fluids: Coexistence data and the approach to the Onsager limit, J. Chem. Phys. 105, 2837 (1996).

[40] G. Odriozola, Revisiting the phase diagram of hard ellipsoids, J. Chem. Phys. 136, 134505 (2012).

[41] C. De Michele, R. Schilling, and F. Sciortino, Dynamics of Uniaxial Hard Ellipsoids, Phys. Rev. Lett. 98, 265702 (2007).

[42] C. De Michele, R. Schilling, and F. Sciortino, Simulation of the dynamics of hard ellipsoids, Philos. Mag. 88, 4117 (2008).

[43] M. Piastra and E. G. Virga, Explicit excluded volume of cylindrically symmetric convex bodies, Phys. Rev. E 91, 062503 (2015).

[44] A. Isihara, Determination of molecular shape by osmotic measurement, J. Chem. Phys. 18, 1446 (1950).

[45] A. Isihara, Theory of anisotropic colloidal solutions, J. Chem. Phys. 19, 1142 (1951).

[46] B. J. Berne and P. Penchukas, Gaussian model potentials for molecular interactions, J. Chem. Phys. 56, 4213 (1971).

[47] E. de Miguel and E. Martín del Río, The isotropic-nematic transition in hard Gaussian overlap fluids, J. Chem. Phys. 115, 9072 (2001). 